\title{
High energy QCD at NLO: from light-cone wave function to JIMWLK evolution
}

\author{
Michael Lublinsky ${ }^{a, b}$ and Yair Mulian ${ }^{a}$ \\ ${ }^{a}$ Department of Physics, Ben-Gurion University of the Negev, \\ Beer-Sheva 84105, Israel \\ ${ }^{b}$ Physics Department, University of Connecticut, \\ 2152 Hillside Road, Storrs, CT 06269-3046, U.S.A. \\ E-mail: lublinm@bgu.ac.il, yair25m@gmail.com
}

ABSTRACT: Soft components of the light cone wave-function of a fast moving projectile hadron is computed in perturbation theory to the third order in QCD coupling constant. At this order, the Fock space of the soft modes consists of one-gluon, two-gluon, and a quark-antiquark states. The hard component of the wave-function acts as a non-Abelian background field for the soft modes and is represented by a valence charge distribution that accounts for non-linear density effects in the projectile. When scattered off a dense target, the diagonal element of the $S$-matrix reveals the Hamiltonian of high energy evolution, the JIMWLK Hamiltonian. This way we provide a new direct derivation of the JIMWLK Hamiltonian at the Next-to-Leading Order.

KeYwords: NLO Computations, QCD Phenomenology

ARXIV EPRINT: 1610.03453 


\section{Contents}

1 Introduction and summary 1

2 Basics of JIMWLK 5

2.1 Light cone QCD Hamiltonian 5

2.2 Field quantisation 6

$\begin{array}{lll}2.3 & \text { Light cone wave-function of a fast hadron } & 7\end{array}$

2.4 Eigenstates of free Hamiltonian 8

2.5 Eikonal approximation for QCD Hamiltonian 9

$\begin{array}{lll}2.6 & \text { Eikonal scattering } & 11\end{array}$

$\begin{array}{lll}2.7 & \text { LO JIMWLK Hamiltonian } & 12\end{array}$

2.8 NLO JIMWLK Hamiltonian 14

3 The light cone wave function at NLO 16

$\begin{array}{ll}3.1 \text { Third order perturbation theory } & 17\end{array}$

$\begin{array}{lll}3.2 & \text { Matrix elements } & 18\end{array}$

$\begin{array}{ll}3.3 \text { Technical aspects of the calculation } & 19\end{array}$

$\begin{array}{ll}3.4 & \text { Computation of the NLO wave function } \\ \end{array}$

$\begin{array}{lll}3.4 .1 & \text { Quark anti-quark state } & 21\end{array}$

3.4.2 Two gluon state 22

$\begin{array}{lll}3.4 .3 & \text { One gluon state } & 25\end{array}$

3.5 The final result 33

4 The next to leading order JIMWLK Hamiltonian $\quad 37$

$\begin{array}{lll}4.1 & \text { Computation of } \Sigma_{q \bar{q}} & 38\end{array}$

$\begin{array}{lll}4.2 & \text { Computation of } \Sigma_{\text {JSSJ }} & 39\end{array}$

4.3 Computation of $\Sigma_{\text {JJSSJ }} \quad 40$

4.4 Computation of $\Sigma_{\text {JJSJ }}$

4.5 Computation of $\Sigma_{\text {JSJ }} \quad 42$

4.6 Computation of $\Sigma_{\text {JJSSJJ }}$ and $\Sigma_{\text {JJJSJ }}$

4.7 Virtual contributions $\quad 45$

4.8 The NLO JIMWLK Hamiltonian assembled 48

$\begin{array}{lll}4.9 & \text { Reduction of the }(\mathrm{LO})^{2} \text { contribution } & 49\end{array}$

A From QCD Lagrangian to light cone QCD Hamiltonian 51

B Deriving the eikonal approximation for LC Hamiltonian 52

$\begin{array}{lll}\text { C Integrals and Fourier transformations } & 53\end{array}$

D Properties of the NLO JIMWLK kernels $\quad 55$ 
F The phase of the wave function $\quad 57$

G Supplementary for section $3 \quad \mathbf{5 8}$

G.1 Supplement for computation of $\left|\psi_{g}^{1}\right\rangle \quad 58$

$\begin{array}{lll}\text { G.2 Supplement for computation of }\left|\psi_{g}^{2}\right\rangle & 59\end{array}$

G.3 Supplement for computation of $\left|\psi_{g}^{3}\right\rangle \quad 61$

G.4 Supplement for computation of $\left|\psi_{g}^{4}\right\rangle$ and $\left|\psi_{g}^{5}\right\rangle \quad 61$

G.5 Supplement for computation of $\left|\psi_{g}^{6}\right\rangle \quad 64$

G.6 Supplement for computation of $\left|\psi_{g}^{7}\right\rangle \quad 67$

$\begin{array}{ll}\text { H Supplement for section } 4 & \mathbf{6 8}\end{array}$

H.1 Supplement for computation of $\Sigma_{q \bar{q}} \quad 68$

$\begin{array}{ll}\text { H.2 Supplement for computation of } \Sigma_{\text {JSSJ }} & 69\end{array}$

$\begin{array}{lll}\text { H.3 Supplement for computation of } \Sigma_{\text {JJSJ }} & 71\end{array}$

H.4 Supplement for computation of $\Sigma_{\text {JJSSJJ }} \quad 72$

\section{Introduction and summary}

The phenomenon of perturbative saturation is believed to occur in high energy hadronic collisions and is most pronounced in processes involving nuclei. The idea dates back to the seminal paper [1]. It has been long known theoretically $[2,3]$ that at high energy gluonic density in a hadron grows exponentially. It was suggested in [1] that this growth does not continue indefinitely, but instead the gluon phase space density saturates when it reaches a critical value of order $1 / \alpha_{s}$.

In the years since the basic idea has been proposed the theoretical understanding of saturation has progressed significantly [6-11]. We now have a first principles QCD formulation of high energy evolution, including the saturation effects (see e.g. [4, 5]). The basic framework was derived in [12-14] — the Balitsky's hierarchy, and in a series of papers [15-22] — the Jalilian-Marian, Iancu, McLerran, Weigert, Leonidov, Kovner (JIMWLK) equation or Colour Glass Condensate (CGC).

The JIMWLK Hamiltonian predicts energy evolution of a hadronic observable $\mathcal{O}$ via functional equation of the form

$$
\frac{d \mathcal{O}}{d Y}=-H_{\text {JIMWLK }} \mathcal{O}
$$

Here the rapidity $Y \sim \ln ($ energy). The JIMWLK Hamiltonian is applicable when one of the colliding particles is dilute (small parton number), such as in forward production in pA collisions at the LHC or in deep inelastic scattering (DIS). While in the dilute-dilute limit, the JIMWLK equation reduces to the linear BFKL equation $[2,3]$ and its BKP extension [23-25], it is free from the famous infrared diffusion problem. Furthermore, the 
major success associated with the JIMWLK is in that it seemingly restores the $s$-channel unitarity of scattering amplitudes (see, however, [26] where the $s$-channel unitarity of the JIMWLK was argued to be incomplete).

Phenomenology based on complete LO JIMWLK has emerged recently [27-31] thanks to its reformulation in terms of stochastic Langevin equation $[29,30]$. Yet, for most phenomenological applications JIMWLK is commonly replaced by the Balitsky-Kovchegov (BK) equation [12-14, 32], which is a mean field/large $N_{c}$ reduction of the JIMWLK. During the last decade, these developments have become a basis for phenomenological studies of saturation physics applied to high energy/low $x$ collision data.

HERA has accumulated a vast amount of precise data in the low $x$ region, which is particularly useful for calibration of gluon distributions used as an input for calculations of less inclusive processes at RHIC and LHC. Several generations of successful saturation-based fits to low $x$ HERA data are available, from the first work [33], to the latest state of the art AAMQS phenomenology [34-36]. These provide comprehensive good quality description of the low $\mathrm{x}$ data, including the latest available combined H1-ZEUS set [37]. However, HERA was not convincing in experimentally confirming the saturation phenomenon.

Even though the main features of CGC are qualitatively understood, quantitative description is still at an unsatisfactory level as most of the studies have been performed to the lowest order (LO) in $\alpha_{s}$. It is, however, known that higher order corrections could be very significant and their inclusion crucial for qualitatively reliable phenomenology. Calculational control enables to discriminate between different approaches, some of which describe experimental data but do not involve saturation, is critically important and is the main challenge. Precision mandates inclusion of next-to-leading order (NLO) corrections to evolution and improvement of fixed order calculations for a variety of observables.

The era of saturation-based phenomenology with NLO precision just started. Among recent progress in fixed order NLO calculations it is important to mention the virtual photon impact factor computed in [38-41] and a series of papers on forward particle production in pA collisions [42-45] (see e.g [46] for a review). The full set of NLO corrections to BK equation was completed in [47] following on the earlier works [48, 49]. When linearised, the NLO BK was shown to coincide with the NLO BFKL computed over a decade earlier [50,51]. Running coupling effects are known to be phenomenologically important. While they do not account for all the NLO effects in the evolution, they do carry important information about high order corrections. Those were analysed in [48, 52-54]. Beyond the NLO accuracy, the BFKL was considered in $[55,56]$.

The NLO extension of the JIMWLK Hamiltonian is imperative for calculation of more general amplitudes, beyond the quark dipole of the BK equation, which determine important experimental observables like single- and double-inclusive particle production. Moreover, precision also mandates going beyond the large $N_{c}$ approximation of the BK equation, which is realised by the JIMWLK. The NLO JIMWLK Hamiltonian was obtained in [57-59] building upon the calculations of [47,60], and in [61] by a direct calculation extending [47]. A conformal part of the Hamiltonian was also deduced in [62]. The NLO JIMWLK contains information both about the NLO BKP $[63,64]$ and on $\alpha_{s}$ corrections to the triple Pomeron vertex. 
While improved equations are known, the key conceptual question remains to be stability of the $\alpha_{s}$ expansion. In the framework of the linear BFKL equation the NLO corrections are big $[50,51]$ and even may lead to negative cross sections. Numerical calculations $[65,66]$ of forward particle production in pA collisions done in the hybrid formalism of $[67,68]$ and including NLO corrections [42-44] displayed similar pathology. The recent study of the NLO BK [69] indicates that the problem is not cured by the non-linear effects. The origin of the problem is understood reasonably well: the LO resummation picks up enhanced logarithmic contributions from the region in the gluon emission phase space, where the underlying approximations break down. These spurious contributions are subtracted in higher orders, thus leading to large negative corrections. To stabilise the expansion, one is normally forced to perform additional collinear resummations introducing kinematical constraints [70-75] or more involved schemes compatible with the DGLAP equation [76-79]. It is clear that a good understanding of a systematic approach to rectifying this problem is urgently needed for reliable phenomenological applications of the NLO BK-JIMWLK framework.

In this paper, we report our contribution to the effort in establishing NLO accuracy in saturation physics. Our results are split into two parts. In the first part, the light cone wave function (LCWF) of a fast moving hadron is computed. More precisely, in a typical Born-Oppenheimer approximation, the Hilbert space of a fast moving projectile is split into a "valence" and a "soft" sectors. The valence gluons have longitudinal momenta greater than some "cutoff" implicitly defined by the collision energy. In the LCWF, these gluons are characterised by correlators of the colour charge densities $\rho^{a}(x)$ ( $x$ is a transverse coordinate).

Soft gluons with momenta smaller than the cutoff do not participate in scattering. Increasing the energy of the hadron increases longitudinal momenta of the soft gluons and they emerge from below the cutoff. Having done so they contribute to physical observables, such as scattering amplitude, particle production etc. This "soft" component of the LCWF at NLO schematically has the form

$$
\begin{aligned}
|\psi\rangle= & \left(1-g_{s}^{2} \kappa_{0} \rho \rho-g_{s}^{4}\left(\delta_{1} \rho \rho+\delta_{2} \rho \rho \rho+\delta_{3} \rho \rho \rho \rho\right) \mid \text { no soft gluons }\right\rangle+ \\
& \left.+\left(g_{s} \kappa_{1} \rho+g_{s}^{3} \epsilon_{1} \rho+g_{s}^{3} \epsilon_{2} \rho \rho+g_{s}^{3} \epsilon_{3} \rho \rho \rho\right) \mid \text { one soft gluon }\right\rangle \\
& \left.\left.+g_{s}^{2}\left(\epsilon_{4} \rho+\epsilon_{5} \rho \rho\right) \mid \text { two soft gluons }\right\rangle+g_{s}^{2} \epsilon_{6} \rho \mid \text { quark }- \text { antiquark }\right\rangle .
\end{aligned}
$$

The coefficient $\kappa_{1}$ is nothing else but the LO Weizsacker Williams gluon emission vertex. Below we compute all the remaining coefficients in the third order perturbation theory in which the valence charges $\rho^{a}$ act as background fields. A major complexity related to this calculation is that these charges are non-commuting operators on the valence Hilbert space forming a local $\mathrm{SU}\left(N_{c}\right)$ algebra. Neither commute the matrix elements of perturbation operators averaged over the soft states. As a result, we had to rederive the perturbation theory while carefully controlling over the ordering between the matrix elements. Most of the coefficients in the first line in (1.2) are computed from the normalisation of the state. Yet, $\delta_{2}$ turns out to be a tricky one: it vanishes in perturbation theory. In our calculation it emerges as a phase of the wavefunction, which could be determined from a constraint 
on applicability of the Born-Oppenheimer approximation. This phase is somewhat similar to the Berry phase, though its nature is apparently rooted in the non-commutativity of $\rho$ rather than being sourced by any topology.

While our calculation and presentation of the results below are fully self-contained, admittedly some of them are not new and could be located in the literature. Particularly, the entire quark sector has been worked out in $[52,53]$ in a formalism that is identical to ours. The coefficients in the second line in (1.2) are related to single inclusive gluon production and we believe that it might be possible to relate $\epsilon_{2}$ with partial result of [80]. The comparison is however not straightforward and we have not pursued it. We also believe that some of our results could be extracted from the LCWF of a dense projectile [81, 82], in which $\alpha_{s}$ corrections enhanced by the density $\rho$ were resummed.

There are many advantages in having the complete LCWF at NLO. First, it provides an alternative method for derivation of the NLO JIMWLK Hamiltonian. Given the complexity of the latter, it is critical to have an independent cross check of the results before moving forward with any phenomenological applications. This constitutes the second part of our paper. Second, the LCWF formalism is possibly a good starting point to reformulate the NLO JIMWLK as stochastic evolution suitable for numeric simulations. Finally, the formalism makes it possible to simultaneously address NLO corrections to the Hamiltonian and to semi-inclusive observables, such as single inclusive production.

As was mentioned above, with increase of collision energy, the soft modes emerge above the cutoff and contribute to scattering cross sections. Thus they are naturally identified with the source of high energy evolution [83]. Let's define the projectile averaged $\hat{S}$-matrix

$$
\Sigma \equiv\langle\psi|\hat{S}-1| \psi\rangle
$$

The averaging is over the soft modes only leaving $\Sigma[\rho]$ as operator on the valence Hilbert space. In fact, $\Sigma$ is a rapidity evolution operator

$$
\Sigma=e^{-\delta Y H_{\mathrm{JIMWLK}}}-1 \simeq-\delta Y H_{\mathrm{JIMWLK}}+\frac{1}{2} \delta Y^{2} H_{\mathrm{JIMWLK}}^{2} \cdots
$$

Here $\delta Y$ is a rapidity interval associated with the longitudinal phase space of the soft modes. The JIMWLK Hamiltonian is expandable in $\alpha_{s}$ :

$$
H_{\mathrm{JIMWLK}}=H_{\mathrm{JIMWLK}}^{L O}\left(\alpha_{s}\right)+H_{\mathrm{JIMWLK}}^{\mathrm{NLO}}\left(\alpha_{s}^{2}\right)+\ldots
$$

Computing (1.3) with the LCWF (1.2) provides a direct method to determine $H_{\text {JIMWLK }}^{\text {NLO }}$ defined as

$$
H_{\text {JIMWLK }} \equiv-\left.\frac{d \Sigma}{d \delta Y}\right|_{\delta Y=0}
$$

This is, however, not how $H_{\mathrm{JIMWLK}}^{\mathrm{NLO}}$ was obtained in [57-59]. There, we only assumed the structure (1.2) without computing any of the coefficients. This structure of the LCWF helped us to identify the most general form of $H_{\text {JIMWLK }}^{\mathrm{NLO}}$. More constraints on the form of the Hamiltonian came from the symmetries of the theory. As discussed in detail in [84], the theory must have $\mathrm{SU}_{L}(N) \times \mathrm{SU}_{R}(N)$ symmetry, which in QCD terms is the gauge symmetry of $\mid$ in $\rangle$ and $\mid$ out $\rangle$ states and two discrete symmetries: the charge conjugation, and another 
$Z_{2}$ symmetry, which in [84] was identified with signature, and can be understood as the combination of charge conjugation and time reversal symmetry [85].

The Hamiltonian in [57] was parametrised by five kernels. They were initially fixed by demanding that the evolution equations for the quark dipole and $\mathrm{SU}(3)$ baryon generated by this Hamiltonian match the ones known from the literature [47, 60]. The Hamiltonian thus constructed was suitable for evolution of gauge invariant operators only. It was later amended in [59] to also include non-gauge invariant pieces. Interestingly, the terms in the Hamiltonian that originate from the previously discussed $\delta_{2}$ were found in [58] thanks to a constraint on conformal invariance of the JIMWLK Hamiltonian in $\mathcal{N}=4$. We quote these results for the Hamiltonian in section 2.8. It will become a benchmark for comparison with our direct calculation.

Below we compute (1.3) with the LCWF (1.2). At $\alpha_{s}^{2}$ order, we find terms that are proportional to $\delta Y$ and terms that are proportional to $\delta Y^{2}$. The former are identified with the NLO JIMWLK Hamiltonian (1.6) and we find a complete agreement with our previous results. The latter are naturally identified with $\left(H_{\mathrm{JIMWLK}}^{L O}\right)^{2}$, which is a non-trivial cross check on our calculation.

This paper is organised as follows. Section 2 provides an introductory overview of the wavefunctional formalism. We start with presenting the QCD Hamiltonian in the light cone gauge, introduce field quantisation and then discuss the eikonal approximation for the LC Hamiltonian and scattering matrix. To prepare the stage, we derive the LO JIMWLK Hamiltonian and also quote the previous result for the NLO JIMWLK. The LCWF is computed in section 3 . The reader who is interested in the end result only may skip most of the section and proceed directly to the final result summarised in section 3.5. Section 4 is devoted to the calculation of $\Sigma$ and extraction of the NLO Hamiltonian. Several appendices contain complementary materials and details of the calculations.

\section{Basics of JIMWLK}

In this section, we review the main elements of high energy QCD in the light cone Hamiltonian formalism.

\subsection{Light cone QCD Hamiltonian}

Our starting point is QCD Hamiltonian in light-cone gauge [86-90]. In light-cone coordinates, four-vectors are $x^{\mu}=\left(x^{+}, x^{-}, \mathbf{x}\right)$, where $x^{+} \equiv x^{0}+x^{3}$ and $x^{-} \equiv x^{0}-x^{3}$ stand for longitudinal, while $\mathbf{x}=\left(x_{1}, x_{2}\right)$ for transverse components. We adopt mostly negative metric [86]. The light-cone gauge:

$$
A^{a+}=A^{a 0}+A^{a 3}=0 .
$$

Derivation of the Hamiltonian in the light cone gauge can be found in many reviews and original publications. For self-completeness of our presentation the derivation is briefly sketched in appendix A:

$$
\begin{aligned}
& H_{\mathrm{LC} Q \mathrm{QCD}} \\
& \quad=\int d x^{-} d^{2} \mathbf{x}\left(\frac{1}{2} \Pi^{a}\left(x^{-}, \mathbf{x}\right) \Pi^{a}\left(x^{-}, \mathbf{x}\right)+\frac{1}{4} F_{i j}^{a}\left(x^{-}, \mathbf{x}\right) F_{i j}^{a}\left(x^{-}, \mathbf{x}\right)+i \bar{\psi} \gamma^{+} D_{+} \psi\right),
\end{aligned}
$$


where the electric and magnetic pieces have the form:

$$
\begin{aligned}
\Pi^{a}\left(x^{-}, \mathbf{x}\right) & \equiv-\frac{1}{\partial^{+}}\left(D_{i}^{a b} \partial^{+} A_{i}^{b}-2 g \psi_{+}^{\dagger} t^{a} \psi_{+}\right), \\
F_{i j}^{a}\left(x^{-}, \mathbf{x}\right) & \equiv \partial_{i} A_{j}^{a}-\partial_{i} A_{j}^{a}-g f^{a b c} A_{i}^{b} A_{j}^{c}
\end{aligned}
$$

$i \in(1,2)$ is a transverse component index; $\psi$ denotes Dirac's 4-component quark spinor, ${ }^{1}$ while $\psi_{+}$is a corresponding 2-component spinor (see appendix A). After substitution of $\Pi^{a}\left(x^{-}, \mathbf{x}\right)$ and $F_{i j}^{a}\left(x^{-}, \mathbf{x}\right)$ in $H_{L C Q C D}$, the result can be written as $H_{L C Q C D}=H_{0}+H_{\text {int }}$, with free Hamiltonian $H_{0}$ given by:

$$
H_{0} \equiv \int d x^{-} d^{2} \mathbf{x}\left(\frac{1}{2}\left(\partial_{i} A_{j}^{a}\right)^{2}+i \psi_{+}^{\dagger} \frac{\partial_{i} \partial_{i}}{\partial^{+}} \psi_{+}\right)
$$

The interaction Hamiltonian $H_{\text {int }}$ reads

$$
\begin{aligned}
H_{\mathrm{int}} \equiv & \int d x^{-} d^{2} \mathbf{x}\left(-g f^{a b c} A_{i}^{b} A_{j}^{c} \partial_{i} A_{j}^{a}+\frac{g^{2}}{4} f^{a b c} f^{a d e} A_{i}^{b} A_{j}^{c} A_{i}^{d} A_{j}^{e}\right. \\
& -g f^{a b c}\left(\partial_{i} A_{i}^{a}\right) \frac{1}{\partial^{+}}\left(A_{j}^{b} \partial^{+} A_{j}^{c}\right)+\frac{g^{2}}{2} f^{a b c} f^{a d e} \frac{1}{\partial^{+}}\left(A_{i}^{b} \partial^{+} A_{i}^{c}\right) \frac{1}{\partial^{+}}\left(A_{j}^{d} \partial^{+} A_{j}^{e}\right) \\
& +2 g^{2} f^{a b c} \frac{1}{\partial^{+}}\left(A_{i}^{b} \partial^{+} A_{i}^{c}\right) \frac{1}{\partial^{+}}\left(\psi_{+}^{\dagger} t^{a} \psi_{+}\right)+2 g^{2} \frac{1}{\partial^{+}}\left(\psi_{+}^{\dagger} t^{a} \psi_{+}\right) \frac{1}{\partial^{+}}\left(\psi_{+}^{\dagger} t^{a} \psi_{+}\right) \\
& -2 g\left(\partial_{i} A_{i}^{a}\right) \frac{1}{\partial^{+}}\left(\psi_{+}^{\dagger} t^{a} \psi_{+}\right)-g \psi_{+}^{\dagger} t^{a}\left(\sigma_{i} \partial_{i}\right) \frac{1}{\partial^{+}}\left(\sigma_{j} A_{j}^{a} \psi_{+}\right)-g \psi_{+}^{\dagger} t^{a} \sigma_{i} A_{i}^{a} \frac{1}{\partial^{+}}\left(\sigma_{j} \partial_{j} \psi_{+}\right) \\
& \left.-i g^{2} \psi_{+}^{\dagger} t^{a} t^{b} \sigma_{i} A_{i}^{a} \frac{1}{\partial^{+}}\left(\sigma_{j} A_{j}^{b} \psi_{+}\right)\right) .
\end{aligned}
$$

$\sigma^{i}$ are Pauli matrices, the matrices $t^{a}$ denote the $\mathrm{SU}\left(N_{c}\right)$ gauge group generators in fundamental representation (the gauge group generators in adjoint representation will be denoted by $T^{a}$ ), which obey the algebra $\left[t^{a}, t^{b}\right]=i f^{a b c} t^{c}$, where $f^{a b c}$ are structure constants of the gauge group.

\section{$2.2 \quad$ Field quantisation}

Quantisation of the fields is performed in usual manner introducing creation/annihilation operators and imposing commutation (anti-commutation) relations among them. For the gauge fields:

$$
A_{i}^{a}(x)=\int_{0}^{\infty} \frac{d k^{+}}{2 \pi} \int \frac{d^{2} \mathbf{k}}{(2 \pi)^{2}} \frac{1}{\sqrt{2 k^{+}}}\left(a_{i}^{a}\left(k^{+}, \mathbf{k}\right) e^{-i k \cdot x}+a_{i}^{a \dagger}\left(k^{+}, \mathbf{k}\right) e^{i k \cdot x}\right) .
$$

The creation and annihilation operators obey the bosonic algebra:

$$
\left[a_{i}^{a}\left(k^{+}, \mathbf{k}\right), a_{j}^{b \dagger}\left(p^{+}, \mathbf{p}\right)\right]=(2 \pi)^{3} \delta^{a b} \delta_{i j} \delta\left(k^{+}-p^{+}\right) \delta^{(2)}(\mathbf{k}-\mathbf{p}) .
$$

Transforming to coordinate space,

$$
a_{i}^{a}\left(k^{+}, \mathbf{k}\right)=\int_{\mathbf{z}} e^{-i \mathbf{k} \cdot \mathbf{z}} a_{i}^{a}\left(k^{+}, \mathbf{z}\right), \quad a_{i}^{a \dagger}\left(k^{+}, \mathbf{k}\right)=\int_{\mathbf{z}} e^{i \mathbf{k} \cdot \mathbf{z}} a_{i}^{a \dagger}\left(k^{+}, \mathbf{z}\right),
$$

\footnotetext{
${ }^{1}$ The flavour index is suppressed in this section.
} 
the commutation relation becomes:

$$
\left[a_{i}^{a}\left(k^{+}, \mathbf{x}\right), a_{j}^{b \dagger}\left(p^{+}, \mathbf{y}\right)\right]=2 \pi \delta^{a b} \delta_{i j} \delta\left(k^{+}-p^{+}\right) \delta^{(2)}(\mathbf{x}-\mathbf{y}) .
$$

For the quark fields:

$$
\psi_{+}^{\alpha}(x)=\sum_{\lambda= \pm \frac{1}{2}} \chi_{\lambda} \int_{0}^{\infty} \frac{d k^{+}}{2 \pi} \int \frac{d^{2} \mathbf{k}}{(2 \pi)^{2}} \frac{1}{\sqrt{2}}\left(b_{\lambda}^{\alpha}\left(k^{+}, \mathbf{k}\right) e^{-i k \cdot x}+d_{\lambda}^{\alpha \dagger}\left(k^{+}, \mathbf{k}\right) e^{i k \cdot x}\right)
$$

The polarisation vectors are:

$$
\begin{array}{rlrl}
\chi_{+\frac{1}{2}} & =\left(\begin{array}{l}
1 \\
0
\end{array}\right), & \chi_{-\frac{1}{2}} & =\left(\begin{array}{l}
0 \\
1
\end{array}\right), \\
\chi_{\lambda_{1}}^{\dagger} I \chi_{\lambda_{2}} & =\delta_{\lambda_{1} \lambda_{2}}, & \chi_{\lambda_{1}}^{\dagger} \sigma^{3} \chi_{\lambda_{2}}=2 \lambda_{1} \delta_{\lambda_{1} \lambda_{2}} .
\end{array}
$$

The anti-commutation relations:

$$
\begin{aligned}
\left\{b_{\lambda_{1}}^{\alpha}\left(k^{+}, \mathbf{k}\right), b_{\lambda_{2}}^{\beta \dagger}\left(p^{+}, \mathbf{p}\right)\right\} & =\left\{d_{\lambda_{1}}^{\alpha}\left(k^{+}, \mathbf{k}\right), d_{\lambda_{2}}^{\beta \dagger}\left(p^{+}, \mathbf{p}\right)\right\} \\
& =(2 \pi)^{3} \delta_{\lambda_{1} \lambda_{2}} \delta^{\alpha \beta} \delta^{(2)}(\mathbf{k}-\mathbf{p}) \delta\left(k^{+}-p^{+}\right) .
\end{aligned}
$$

Transforming the fields to coordinate space a la (2.8):

$$
\begin{aligned}
\left\{b_{\lambda_{1}}^{\alpha}\left(k^{+}, \mathbf{x}\right), b_{\lambda_{2}}^{\beta \dagger}\left(p^{+}, \mathbf{y}\right)\right\} & =\left\{d_{\lambda_{1}}^{\alpha}\left(k^{+}, \mathbf{x}\right), d_{\lambda_{2}}^{\beta \dagger}\left(p^{+}, \mathbf{y}\right)\right\} \\
& =2 \pi \delta_{\lambda_{1} \lambda_{2}} \delta^{\alpha \beta} \delta^{(2)}(\mathbf{x}-\mathbf{y}) \delta\left(k^{+}-p^{+}\right) .
\end{aligned}
$$

Inserting the field expansions (2.6) and (2.10) into (2.4), the free Hamiltonian becomes:

$$
\begin{aligned}
H_{0}=\int_{0}^{\infty} \frac{d k^{+}}{2 \pi} & \int \frac{d^{2} \mathbf{k}}{(2 \pi)^{2}} \frac{\mathbf{k}^{2}}{2 k^{+}}\left(a_{i}^{a \dagger}\left(k^{+}, \mathbf{k}\right) a_{i}^{a}\left(k^{+}, \mathbf{k}\right)\right. \\
& \left.+\sum_{\lambda}\left[b_{\lambda}^{\alpha \dagger}\left(k^{+}, \mathbf{k}\right) b_{\lambda}^{\alpha}\left(k^{+}, \mathbf{k}\right)-d_{\lambda}^{\alpha}\left(k^{+}, \mathbf{k}\right) d_{\lambda}^{\alpha \dagger}\left(k^{+}, \mathbf{k}\right)\right]\right),
\end{aligned}
$$

from which the dispersion relation for free quarks and gluons is $E_{k}=\frac{\mathbf{k}^{2}}{2 k^{+}}$. The interacting part of the Hamiltonian will be quantised in subsection 2.5.

\subsection{Light cone wave-function of a fast hadron}

Consider a fast moving projectile hadron at some rapidity $Y_{0}$. Its LCWF $|\Psi\rangle_{Y_{0}}$ is an eigenfunction of $H_{L C Q C D}$. It is convenient to split the modes in the LCWF in accord to their longitudinal momenta, introducing a longitudinal cutoff $\Lambda$, which is implicitly related to the energy of the target hadron (see [91] for review of the formalism). We further assume that the soft modes in the LCWF whose longitudinal momenta $k^{+}$is smaller than $\Lambda$ are not energetic enough to contribute significantly to the scattering process (their contribution is power suppressed by the collision energy). The hard modes with $k^{+}>\Lambda$ are also referred as valence modes. 


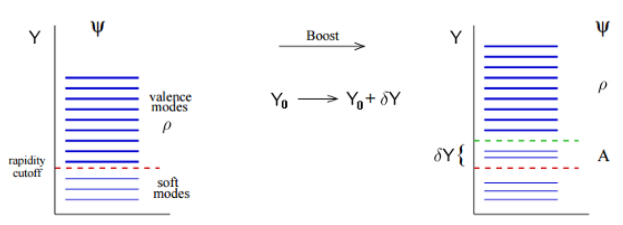

Figure 1. The LCWFs before and after boost. Here $\rho$ stands for the colour charge density of the valence modes, and A for the soft gluon field.

Total energy of the collision can be increased by boosting the projectile: $\mathrm{Y}=\mathrm{Y}_{0}+\delta \mathrm{Y}$, whereas $\delta \mathrm{Y}$ is a boost parameter. By boosting the projectile, all the longitudinal momenta of partons in the LCWF get shifted up (figure 1):

$$
k^{+} \longrightarrow e^{\delta \mathrm{Y}} k^{+} \text {. }
$$

As a result, some of the soft modes that lived below the cutoff $\Lambda$ get lifted above $\Lambda$ and start to contribute to the scattering process. After the boost, these modes occupy a window $\Lambda<k^{+}<\Lambda e^{\delta Y}$. The valence modes get shifted above this window.

Ignoring the modes below $\Lambda$, the LCWF $|\Psi\rangle_{Y_{0}}$ before the boost is dominated by the valence modes only, whose distribution is approximately independent of their longitudinal momenta.

$$
|\Psi\rangle_{Y_{0}}=|v\rangle \text {. }
$$

Here $|v\rangle$ represents a valence state with no soft gluons (vaccuum of soft gluons). We assume that this LCWF is known. The LCWF after the boost is

$$
|\Psi\rangle_{Y}=|\psi\rangle \otimes|v\rangle,
$$

where $|\psi\rangle$ denotes the soft part of the wave function living in the window $\Lambda<k^{+}<\Lambda e^{\delta \curlyvee}$. The factorisation (2.18) is a typical Born-Oppenheimer approximation. Our goal below will be to find $|\psi\rangle$ by perturbatively diagonalising $H_{L C Q C D}$ on the Hilbert space of the soft modes, assuming the valence sector is known and frozen.

\subsection{Eigenstates of free Hamiltonian}

We denote by $|0\rangle$ the vacuum state of the free Hamiltonian $H_{0}$ on the soft Hilbert space. The energy of this state will be set to zero, $E_{0}=0$. The vacuum is defined as:

$$
a_{i}^{a}\left(k^{+}, \mathbf{k}\right)|0\rangle=0, \quad b_{i}^{\alpha}\left(k^{+}, \mathbf{k}\right)|0\rangle=0, \quad d_{i}^{\alpha}\left(k^{+}, \mathbf{k}\right)|0\rangle=0,
$$

for any $\Lambda<k^{+}<e^{\delta \curlyvee} \Lambda$. The soft vacuum $|0\rangle$ should be understood as $|0\rangle \otimes|v\rangle$ and, because of its valence component, it differs from the QCD vacuum. As explained above, our prime objective in the next section will be to develop a perturbation theory for this soft vacuum state. Three eigenstates of $H_{0}$ will emerge in the NLO calculation below:

- One gluon state.

$$
\left|g_{i}^{a}(k)\right\rangle \equiv \frac{a_{i}^{a \dagger}(k)}{(2 \pi)^{3 / 2}}|0\rangle \quad \text { or } \quad\left|g_{i}^{a}\left(k^{+}, \mathbf{z}\right)\right\rangle \equiv \frac{a_{i}^{a \dagger}\left(k^{+}, \mathbf{z}\right)}{(2 \pi)^{1 / 2}}|0\rangle,
$$


These is a normalised state with the normalisation

$$
\left\langle g_{j}^{b}(p) \mid g_{i}^{a}(k)\right\rangle=\delta^{a b} \delta_{i j} \delta^{(2)}(\mathbf{k}-\mathbf{p}) \delta\left(k^{+}-p^{+}\right)
$$

and

$$
\left\langle g_{j}^{b}\left(p^{+}, \mathbf{z}^{\prime}\right) \mid g_{i}^{a}\left(k^{+}, \mathbf{z}\right)\right\rangle=\delta^{a b} \delta_{i j} \delta^{(2)}\left(\mathbf{z}-\mathbf{z}^{\prime}\right) \delta\left(k^{+}-p^{+}\right) .
$$

This state has the energy $E_{g}(k)=\frac{\mathbf{k}^{2}}{2 k^{+}}$.

\section{- Two gluon state.}

$$
\begin{aligned}
\left|g_{i}^{a}(k) g_{j}^{b}(p)\right\rangle & \equiv \frac{a_{i}^{a \dagger}(k) a_{j}^{b \dagger}(p)}{(2 \pi)^{3}}|0\rangle \quad \text { or } \\
\left|g_{i}^{a}\left(k^{+}, \mathbf{z}\right) g_{j}^{b}\left(p^{+}, \mathbf{z}^{\prime}\right)\right\rangle & \equiv \frac{a_{i}^{a \dagger}\left(k^{+}, \mathbf{z}\right) a_{j}^{b \dagger}\left(p^{+}, \mathbf{z}^{\prime}\right)}{2 \pi}|0\rangle .
\end{aligned}
$$

with the energy $E_{g g}(k, p) \equiv \frac{\mathbf{k}^{2}}{2 k^{+}}+\frac{\mathbf{p}^{2}}{2 p^{+}}$.

- Quark anti-quark state.

$$
\begin{aligned}
\left|\bar{q}_{\lambda_{2}}^{\beta}(p) q_{\lambda_{1}}^{\alpha}(k)\right\rangle & \equiv \frac{b_{\lambda_{1}}^{\alpha \dagger}(k) d_{\lambda_{2}}^{\beta \dagger}(p)}{(2 \pi)^{3}}|0\rangle \text { or } \\
\left|\bar{q}_{\lambda_{2}}^{\beta}\left(p^{+}, \mathbf{z}^{\prime}\right) q_{\lambda_{1}}^{\alpha}\left(k^{+}, \mathbf{z}\right)\right\rangle & \equiv \frac{b_{\lambda_{1}}^{\alpha \dagger}\left(k^{+}, \mathbf{z}\right) d_{\lambda_{2}}^{\beta \dagger}\left(p^{+}, \mathbf{z}^{\prime}\right)}{2 \pi}|0\rangle,
\end{aligned}
$$

with the energy $E_{q \bar{q}}(k, p)=\frac{\mathbf{k}^{2}}{2 k^{+}}+\frac{\mathbf{p}^{2}}{2 p^{+}}$.

\subsection{Eikonal approximation for QCD Hamiltonian}

In section 2.3, we split the Hilbert space into soft and valence modes and introduced a BornOppenheimer approximation for the LCWF. Accordingly, we have to split the Hamiltonian $H_{\text {int }}$ as defined by (2.5) into "soft" and "valence". First, we introduce field decomposition: $A_{i}^{a}(x)=\underline{A}_{i}^{a}(x)+\bar{A}_{i}^{a}(x)$ and $\psi_{+}^{\alpha}(x)=\underline{\psi}_{+}^{\alpha}(x)+\bar{\psi}_{+}^{\alpha}(x)$ where the underlined fields contain only modes with longitudinal momenta in the interval $k^{+} \in\left(\Lambda, e^{\delta \curlyvee} \Lambda\right)$ (soft modes) while the fields with the bar contain modes with $k^{+}>e^{\delta \curlyvee} \Lambda$ (valence modes). Substitution of this decomposition into $H_{\text {int }}$ is done in appendix B.

Our next step is to introduce an eikonal approximation for the interaction Hamiltonian. A typical longitudinal momentum of a valence mode is much larger than that of a soft mode. Consequently, all the terms in $H_{\text {int }}$ that are suppressed by the ratio of soft to valence longitudinal momenta can be systematically ignored. Keeping these terms would introduce non-eikonal power suppressed corrections to our calculation. Example of terms in the Hamiltonian which are neglected $\frac{1}{\partial^{+}}\left(\underline{A}_{j}^{b} \partial^{+} \bar{A}_{j}^{c}\right)$ or $\frac{1}{\partial^{+}}\left(\underline{\psi}_{+}^{\dagger} t^{a} \bar{\psi}_{+}\right)$. The action of $\frac{1}{\partial^{+}}$is equivalent to division by a very large valence momentum (i.e. $\frac{1}{p^{+}} \rightarrow 0$ for any $p^{+}>e^{\delta \curlyvee} \Lambda$ ).

In appendix $\mathrm{B}$, we first split the modes between soft and valence in $H_{\text {int }}$ and then employ the eikonal approximation. The result is

$$
H_{\mathrm{int}}=H_{g}+H_{g q q}+H_{g g g}+H_{g g g g}+H_{g g q q}+H_{q q-\mathrm{inst}}+H_{g g-\mathrm{inst}}+H_{g q}+H_{V} .
$$


The first eight contributions defined by (B.5) - (B.12), account for soft-soft and soft-valence interactions, while $H_{V}$ involves valence-valence interactions only.

Here $\rho^{a}(-\mathbf{p})$ denotes the total valence current (charge density operator) which acts as a background for the soft fields, and is defined by:

$$
\rho^{a}(-\mathbf{p}) \equiv \rho_{g}^{a}(-\mathbf{p})+\rho_{q \bar{q}}^{a}(-\mathbf{p})
$$

with

$$
\rho_{g}^{a}(-\mathbf{p}) \equiv-i f^{a b c} \int_{e^{\delta \curlyvee \Lambda}}^{\infty} \frac{d k^{+}}{2 \pi} \int \frac{d^{2} \mathbf{k}}{(2 \pi)^{2}} a_{j}^{\dagger b}\left(k^{+}, \mathbf{k}\right) a_{j}^{c}\left(k^{+}, \mathbf{k}+\mathbf{p}\right),
$$

and

$$
\rho_{q \bar{q}}^{a}(-\mathbf{p}) \equiv t_{\alpha \beta}^{a} \int_{e^{\delta \curlyvee}}^{\infty} \frac{d k^{+}}{2 \pi} \int \frac{d^{2} \mathbf{k}}{(2 \pi)^{2}}\left(b_{\lambda}^{\alpha \dagger}\left(k^{+}, \mathbf{k}-\mathbf{p}\right) b_{\lambda}^{\beta}\left(k^{+}, \mathbf{k}\right)+d_{\lambda}^{\beta}\left(k^{+}, \mathbf{k}\right) d_{\lambda}^{\alpha \dagger}\left(k^{+}, \mathbf{k}-\mathbf{p}\right)\right) .
$$

The valence current $\rho^{a}(\mathbf{p})$ satisfies the $\mathrm{SU}\left(N_{c}\right)$ algebra:

$$
\left[\rho^{a}(\mathbf{x}), \rho^{b}(\mathbf{y})\right]=i f^{a b c} \rho^{c}(\mathbf{x}) \delta^{(2)}(\mathbf{x}-\mathbf{y}) ; \quad\left[\rho^{a}(\mathbf{k}), \rho^{b}(\mathbf{p})\right]=i f^{a b c} \rho^{c}(\mathbf{k}+\mathbf{p}) .
$$

where $\rho^{a}(\mathbf{x})$ is a Fourier transform of $\rho^{a}(\mathbf{p})$ :

$$
\rho^{a}(\mathbf{x})=\frac{1}{(2 \pi)^{2}} \int d^{2} \mathbf{k} e^{i \mathbf{k} \cdot \mathbf{x}} \rho^{a}(-\mathbf{k}) ; \quad \rho^{a}(-\mathbf{k})=\int d^{2} \mathbf{x} e^{-i \mathbf{k} \cdot \mathbf{x}} \rho^{a}(\mathbf{x})
$$

As a preparation for the next section, it is useful to express (B.5) - (B.9) in terms of the creation and annihilation operators. This is done by employing the expansions (2.6) and (2.10). Only the terms, which would yield a non-trivial contribution when inserted between the three states defined in section 2.3, and the soft vacuum, will be kept. We omit further details and write the final results,

$$
\begin{aligned}
\mathrm{H}_{g}= & \int_{\Lambda}^{e^{\delta \curlyvee} \Lambda} \frac{d k^{+}}{2 \pi} \int \frac{d^{2} \mathbf{k}}{(2 \pi)^{2}} \frac{g \mathbf{k}^{i}}{\sqrt{2}\left|k^{+}\right|^{3 / 2}}\left[a_{i}^{a \dagger}\left(k^{+}, \mathbf{k}\right) \rho^{a}(-\mathbf{k})+a_{i}^{a}\left(k^{+}, \mathbf{k}\right) \rho^{a}(\mathbf{k})\right], \\
\mathrm{H}_{g q q}= & \sum_{\lambda_{1}, \lambda_{2}= \pm \frac{1}{2}} \int_{\Lambda}^{e^{\delta \curlyvee} \Lambda} \frac{d k^{+}}{2 \pi} \frac{d p^{+}}{2 \pi} d q^{+} \int \frac{d^{2} \mathbf{k}}{(2 \pi)^{2}} \frac{d^{2} \mathbf{p}}{(2 \pi)^{2}} d^{2} \mathbf{q} \frac{g t_{\alpha \beta}^{a}}{2 \sqrt{2 k^{+}}} \delta^{(3)}(k-p-q) \Gamma_{\lambda_{1} \lambda_{2}}^{i} \\
& \times\left(a_{i}^{a}\left(k^{+}, \mathbf{k}\right) b_{\lambda_{1}}^{\alpha \dagger}\left(p^{+}, \mathbf{p}\right) d_{\lambda_{2}}^{\beta \dagger}\left(q^{+}, \mathbf{q}\right)+\text { h.c. }\right),
\end{aligned}
$$


with

$$
\begin{aligned}
\Gamma_{\lambda_{1} \lambda_{2}}^{i}= & \chi_{\lambda_{1}}^{\dagger}\left[\frac{2 \mathbf{k}^{i}}{k^{+}}-\frac{\sigma \cdot \mathbf{p}}{p^{+}} \sigma^{i}-\sigma^{i} \frac{\sigma \cdot \mathbf{q}}{q^{+}}\right] \chi_{\lambda_{2}} \\
\mathrm{H}_{g g g}= & -\int_{\Lambda}^{e^{\delta \curlyvee}} \frac{d k^{+}}{2 \pi} \frac{d p^{+}}{2 \pi} d q^{+} \int \frac{d^{2} \mathbf{k}}{(2 \pi)^{2}} \frac{d^{2} \mathbf{p}}{(2 \pi)^{2}} d^{2} \mathbf{q} \frac{i g f^{a b c}}{2 \sqrt{2 k^{+} p^{+} q^{+}}} \\
& \times\left[\left(\mathbf{q}^{i}-\frac{q^{+}}{p^{+}+q^{+}} \mathbf{k}^{i}\right) a_{i}^{a}(k) a_{j}^{b \dagger}(p) a_{j}^{c \dagger}(q) \delta^{(3)}(-k+p+q)\right. \\
& \left.+\left(\mathbf{q}^{i}+\frac{p^{+}+q^{+}}{q^{+}-p^{+}} \mathbf{k}^{i}\right) a_{i}^{a \dagger}(k) a_{j}^{b \dagger}(p) a_{j}^{c}(q) \delta^{(3)}(k+p-q),+ \text { h.c. }\right] \\
\mathrm{H}_{g g-\text { inst }}= & \int_{\Lambda}^{e^{\delta \curlyvee}} \frac{d p^{+}}{2 \pi} \frac{d q^{+}}{2 \pi} \int \frac{d^{2} \mathbf{p}}{(2 \pi)^{2}} \frac{d^{2} \mathbf{q}}{(2 \pi)^{2}} \frac{i g^{2} f^{a b c}}{\sqrt{p^{+} q^{+}}}\left(\frac{q^{+} \rho^{a}(-\mathbf{p}-\mathbf{q})}{2\left(p^{+}+q^{+}\right)^{2}} a_{i}^{b \dagger}\left(p^{+}, \mathbf{p}\right) a_{i}^{c \dagger}\left(q^{+}, \mathbf{q}\right)\right. \\
& \left.- \text { h.c. }-\frac{\left(p^{+}+q^{+}\right) \rho^{a}(-\mathbf{p}+\mathbf{q})}{2\left(p^{+}-q^{+}\right)^{2}} a_{i}^{b \dagger}\left(p^{+}, \mathbf{p}\right) a_{i}^{c}\left(q^{+}, \mathbf{q}\right)\right) \\
\mathrm{H}_{q q-\text { inst }}= & \sum_{\lambda_{1}, \lambda_{2}} \int_{\Lambda}^{e^{\delta \curlyvee} \Lambda} \frac{d p^{+}}{2 \pi} \frac{d q^{+}}{2 \pi} \int \frac{d^{2} \mathbf{p}}{(2 \pi)^{2}} \frac{d^{2} \mathbf{q}}{(2 \pi)^{2}} \frac{g^{2} t_{\alpha \beta}^{a} \rho^{a}(-\mathbf{p}-\mathbf{q}) \chi_{\lambda_{1}}^{\dagger} \chi_{\lambda_{2}}}{\left(p^{+}+q^{+}\right)^{2}} \\
& \times\left[b_{\lambda_{1}}^{\alpha \dagger}\left(p^{+}, \mathbf{p}\right) d_{\lambda_{2}}^{\beta \dagger}\left(q^{+}, \mathbf{q}\right)+\text { h.c. }\right]
\end{aligned}
$$

\subsection{Eikonal scattering}

At very high collision energies, a fast projectile parton moves along its straight-line classical trajectory and the only scattering effect is the eikonal phase factor acquired along its propagation path. We assume this approximation to be valid for all partons with longitudinal momenta above $\Lambda$. The $\hat{S}$ matrix operator is diagonal in the coordinate space:

$$
\begin{gathered}
\hat{S} a_{i}^{a \dagger}\left(x^{+}, \mathbf{x}\right)|0\rangle=S_{A}^{a b}(\mathbf{x}) a_{i}^{b \dagger}\left(x^{+}, \mathbf{x}\right)|0\rangle \\
\hat{S} b_{i}^{\alpha \dagger}\left(x^{+}, \mathbf{x}\right)|0\rangle=S^{\beta \alpha}(\mathbf{x}) b_{i}^{\beta \dagger}\left(x^{+}, \mathbf{x}\right)|0\rangle, \quad \hat{S} d_{i}^{\alpha \dagger}\left(x^{+}, \mathbf{x}\right)|0\rangle=S^{\alpha \beta}(\mathbf{x}) d_{i}^{\beta \dagger}\left(x^{+}, \mathbf{x}\right)|0\rangle
\end{gathered}
$$

$S_{A}^{a b}(\mathbf{x})$ and $S^{\alpha \beta}(\mathbf{x})$ are Wilson lines in the adjoint and fundamental representations,

$$
\begin{aligned}
& S_{A}^{a b}(\mathbf{x})=\left[\mathcal{P} \exp \left\{i g \int d x^{+} T^{c} A^{-c}\left(x^{+}, \mathbf{x}\right)\right\}\right]^{a b}, \\
& S^{\alpha \beta}(\mathbf{x})=\left[\mathcal{P} \exp \left\{i g \int d x^{+} t^{c} A^{-c}\left(x^{+}, \mathbf{x}\right)\right\}\right]^{\alpha \beta} .
\end{aligned}
$$

The valence part scattering: $\hat{S}|v\rangle=|S v\rangle$. The operator $\rho$ gets affected by the scattering as well. As explained in [83], it is possible to express the action of $\rho$ on the state $|S v\rangle$ as a functional Lie derivative:

$$
\rho_{g}^{a}(\mathbf{x}) \hat{S}|v\rangle=J_{R, a d j}^{a}(\mathbf{x})|\hat{S} v\rangle, \quad \quad \hat{S} \rho_{g}^{a}(\mathbf{x})|v\rangle=J_{L, a d j}^{a}(\mathbf{x})|\hat{S} v\rangle,
$$


which act as right and left rotations on the Wilson lines $S$,

$$
\begin{aligned}
& J_{R, a d j}^{a}(\mathbf{x}) \equiv-\operatorname{tr}\left[S_{A}(\mathbf{x}) T^{a} \frac{\delta}{\delta S_{A}^{\dagger}(\mathbf{x})}\right] \\
& J_{L, a d j}^{a}(\mathbf{x}) \equiv\left[S_{A}(\mathbf{x}) J_{R}(\mathbf{x})\right]^{a}=-\operatorname{tr}\left[T^{a} S_{A}(\mathbf{x}) \frac{\delta}{\delta S_{A}^{\dagger}(\mathbf{x})}\right] .
\end{aligned}
$$

In a similar manner we can also express $\rho_{q \bar{q}}$ :

$$
\rho_{q \bar{q}}^{a}(\mathbf{x}) \hat{S}|v\rangle=J_{R, F}^{a}(\mathbf{x})|\hat{S} v\rangle, \quad \hat{S} \rho_{q \bar{q}}(\mathbf{x})|v\rangle=J_{L, F}^{a}(\mathbf{x})|\hat{S} v\rangle .
$$

with

$$
\begin{aligned}
& J_{R, F}^{a}(\mathbf{x}) \equiv \operatorname{tr}\left[\frac{\delta}{\delta S^{T}(\mathbf{x})} S(\mathbf{x}) t^{a}\right]-\operatorname{tr}\left[\frac{\delta}{\delta S^{*}(\mathbf{x})} t^{a} S^{\dagger}(\mathbf{x})\right] \\
& J_{L, F}^{a}(\mathbf{x}) \equiv \operatorname{tr}\left[\frac{\delta}{\delta S^{T}(\mathbf{x})} t^{a} S(\mathbf{x})\right]-\operatorname{tr}\left[\frac{\delta}{\delta S^{*}(\mathbf{x})} S^{\dagger}(\mathbf{x}) t^{a}\right] .
\end{aligned}
$$

The following relations will be useful for the NLO calculations:

$$
\begin{aligned}
& \rho^{a}(\mathbf{y}) \rho^{b}(\mathbf{x}) \hat{S}|v\rangle=J_{R}^{b}(\mathbf{x}) J_{R}^{a}(\mathbf{y})|\hat{S} v\rangle, \\
& \rho^{a}(\mathbf{y}) \hat{S} \rho^{b}(\mathbf{x})|v\rangle=J_{L}^{b}(\mathbf{x}) J_{R}^{a}(\mathbf{y})|\hat{S} v\rangle, \\
& \hat{S} \rho^{a}(\mathbf{y}) \rho^{b}(\mathbf{x})|v\rangle=J_{L}^{a}(\mathbf{y}) J_{L}^{b}(\mathbf{x})|\hat{S} v\rangle .
\end{aligned}
$$

The algebra of the operators $J_{L / R}^{a}(\mathbf{x}) \equiv J_{L / R, F}^{a}(\mathbf{x})+J_{L / R, a d j}^{a}(\mathbf{x})$ follows from that of $\rho$ :

$$
\left[J_{L}^{a}(\mathbf{x}), J_{L}^{b}(\mathbf{y})\right]=-i f^{a b c} J_{L}^{c}(\mathbf{x}) \delta(\mathbf{x}-\mathbf{y}) ; \quad\left[J_{R}^{a}(\mathbf{x}), J_{R}^{b}(\mathbf{y})\right]=i f^{a b c} J_{R}^{c}(\mathbf{x}) \delta(\mathbf{x}-\mathbf{y}),
$$

while $\left[J_{L}^{a}(\mathbf{x}), J_{R}^{b}(\mathbf{y})\right]=0$. As mentioned above, $J_{L / R}$ act on $S$ as left and right rotations:

$$
\left[J_{L}^{c}(\mathbf{x}), S_{A}^{a b}(\mathbf{z})\right]=-i f^{c a d} S_{A}^{d b}(\mathbf{z}) \delta(\mathbf{x}-\mathbf{z}) ; \quad\left[J_{R}^{c}(\mathbf{x}), S_{A}^{a b}(\mathbf{z})\right]=i f^{c b d} S_{A}^{a d}(\mathbf{z}) \delta(\mathbf{x}-\mathbf{z}) .
$$

These algebra will be used in section 4.9 , where we subtract the $(L O)^{2}$ effects.

\subsection{LO JIMWLK Hamiltonian}

To warm up, we will follow the approach of [91] and derive the LO JIMWLK Hamiltonian using the LCWF formalism. At leading order, the only relevant process is one soft gluon emission by valence current, which is displayed in figure 2 .

The soft part of the wave-function is given by the first order perturbation theory:

$$
\left|\psi^{L O}\right\rangle=\mathcal{N}^{L O}|0\rangle-|i\rangle \frac{\left\langle i\left|H_{\text {int }}\right| 0\right\rangle}{E_{i}},
$$

where $\mathcal{N}^{L O}$ is determined by the normalisation condition, $\left\langle\psi^{L O} \mid \psi^{L O}\right\rangle=1$ :

$$
\mathcal{N}^{L O} \equiv 1-\frac{\left|\left\langle i\left|H_{\mathrm{int}}\right| 0\right\rangle\right|^{2}}{2 E_{i}^{2}} .
$$




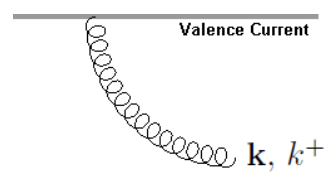

Figure 2. Soft gluon emission with momentum $k=\left(\mathbf{k}, k^{+}\right)$from a valence current $\rho$ at order $g$. The emission vertex is defined by $\left|\psi^{L O}\right\rangle$.

At order $g$, there is only one state, $\left|g_{i}^{a}(k)\right\rangle$ that contributes to (2.47). The only nonvanishing matrix-element is that of $H_{g},(2.29)$ :

$$
\left\langle g_{i}^{a}(k)\left|H_{g}\right| 0\right\rangle=\frac{g \mathbf{k}^{i} \rho^{a}(-\mathbf{k})}{4 \pi^{3 / 2}\left|k^{+}\right|^{3 / 2}} .
$$

Therefore, (2.47) becomes:

$$
\left|\psi^{L O}\right\rangle=\mathcal{N}^{L O}|0\rangle-\int_{\Lambda}^{e^{\delta \curlyvee} \Lambda} d k^{+} \int d^{2} \mathbf{k} \frac{g \mathbf{k}^{i}}{2 \pi^{3 / 2} \sqrt{k^{+}} \mathbf{k}^{2}} \rho^{a}(-\mathbf{k})\left|g_{i}^{a}(k)\right\rangle .
$$

We will proceed by calculating the Fourier transformation of $\left|\psi^{L O}\right\rangle$. The coordinate space representation will make it possible to compute the scattered wave function, since, as explained in the previous section, the $S$-matrix is diagonal in the $x$-space. The charge densities $\rho^{a}(-\mathbf{k})$ are Fourier transformed according to $(2.28)$, and the transformation to the coordinates space is done using (C.21). The wave function becomes:

$$
\left|\psi^{L O}\right\rangle=\mathcal{N}^{L O}|0\rangle+\int_{\Lambda}^{e^{\delta \curlyvee} \Lambda} \frac{d k^{+}}{\sqrt{k^{+}}} \int_{\mathbf{x}, \mathbf{z}} \frac{i g X^{i}}{2 \pi^{3 / 2} X^{2}} \rho^{a}(\mathbf{x})\left|g_{i}^{a}\left(k^{+}, \mathbf{z}\right)\right\rangle,
$$

where $X^{i} \equiv \mathbf{x}^{i}-\mathbf{z}^{i}$. From (2.48):

$$
\mathcal{N}^{L O}=1-\frac{g^{2}}{8 \pi^{3}} \int_{\Lambda}^{e^{\delta \curlyvee} \Lambda} \frac{d k^{+}}{k^{+}} \int_{\mathbf{x}, \mathbf{y}, \mathbf{z}} \frac{X \cdot Y}{X^{2} Y^{2}} \rho^{a}(\mathbf{y}) \rho^{a}(\mathbf{x})+\mathcal{O}\left(g^{4}\right),
$$

where $Y^{i} \equiv \mathbf{y}^{i}-\mathbf{z}^{i}$. The scattered wave function reads:

$$
\begin{aligned}
\hat{S}\left|\psi^{L O}\right\rangle= & \left(1-\frac{g^{2}}{8 \pi^{3}} \int_{\Lambda}^{e^{\delta \curlyvee} \Lambda} \frac{d k^{+}}{k^{+}} \int_{\mathbf{x}, \mathbf{y}, \mathbf{z}} \frac{X \cdot Y}{X^{2} Y^{2}} \hat{S} \rho^{a}(\mathbf{y}) \rho^{a}(\mathbf{x})\right)|0\rangle \\
& +\int_{\Lambda}^{e^{\delta \curlyvee} \Lambda} \frac{d k^{+}}{\sqrt{k^{+}}} \int_{\mathbf{x}, \mathbf{z}} \frac{i g X^{i}}{2 \pi^{3 / 2} X^{2}} \hat{S} \rho^{a}(\mathbf{x})\left|g_{i}^{a}\left(k^{+}, \mathbf{z}\right)\right\rangle .
\end{aligned}
$$

By inserting the wave functions before and after the scattering process in (1.3) and using the relations (2.44), we arrive at:

$$
\begin{aligned}
\Sigma^{L O} & \equiv\left\langle\psi^{L O}|\hat{S}-1| \psi^{L O}\right\rangle \\
& =-\frac{\alpha_{s}}{2 \pi^{2}} \delta \mathbf{Y} \int_{\mathbf{x}, \mathbf{y}, \mathbf{z}} \frac{X \cdot Y}{X^{2} Y^{2}}\left[J_{L}^{a}(\mathbf{x}) J_{L}^{a}(\mathbf{y})+J_{R}^{a}(\mathbf{x}) J_{R}^{a}(\mathbf{y})-2 J_{L}^{a}(\mathbf{x}) S_{A}^{a b}(\mathbf{z}) J_{R}^{b}(\mathbf{y})\right] .
\end{aligned}
$$




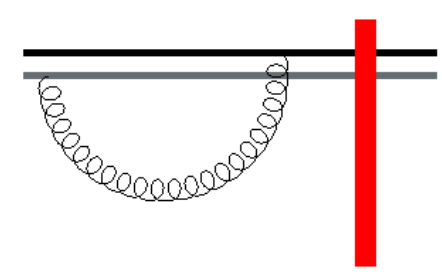

(a)

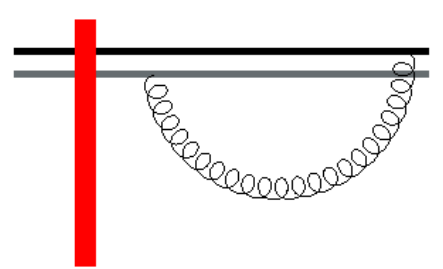

(b)

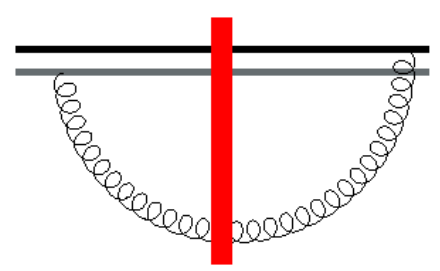

(c)

Figure 3. Figures $(a)$ and $(b)$ correspond to the contributions from the normalisation of the wave function, while the figure $(c)$ illustrates the real gluon scattering contribution. The instantaneous shockwave interaction between the projectile and the target is represented by the thick (red) line. Each operator $J_{L} / J_{R}$ in the JIMWLK Hamiltonian correspond to the emission vertice of a soft gluon from the valence current.

Following the definition (1.6), the LO Hamiltonian is $H^{L O} \equiv-\frac{\delta \Sigma^{L O}}{\delta \mathrm{Y}}$, which gives (see figure 3 for schematic illustration of the LO Hamiltonian)

$$
H_{\mathrm{JIMWLK}}^{L O}=\frac{\alpha_{s}}{2 \pi^{2}} \int_{\mathbf{x}, \mathbf{y}, \mathbf{z}} \frac{X \cdot Y}{X^{2} Y^{2}}\left[J_{L}^{a}(\mathbf{x}) J_{L}^{a}(\mathbf{y})+J_{R}^{a}(\mathbf{x}) J_{R}^{a}(\mathbf{y})-2 J_{L}^{a}(\mathbf{x}) S_{A}^{a b}(\mathbf{z}) J_{R}^{b}(\mathbf{y})\right] .
$$

The LO Hamiltonian is invariant under $S U_{L}\left(N_{c}\right) \times S U_{R}\left(N_{c}\right)$ rotations, which reflect gauge invariance of scattering amplitudes. In addition, the Hamiltonian is invariant under $Z_{2}$ transformation, $S \rightarrow S^{\dagger}$ together with $J_{L} \rightarrow-J_{R}$, which in [84] was identified as signature, and the charge conjugation symmetry $S \rightarrow S^{*}$.

\subsection{NLO JIMWLK Hamiltonian}

The NLO JIMWLK Hamiltonian deduced in [57] is:

$$
\begin{aligned}
& H_{\mathrm{JIMWLK}}^{\mathrm{NLO}}=\int_{\mathbf{x}, \mathbf{y}, \mathbf{z}} K_{\mathrm{JSJ}}(\mathbf{x}, \mathbf{y}, \mathbf{z})\left[J_{L}^{a}(\mathbf{x}) J_{L}^{a}(\mathbf{y})+J_{R}^{a}(\mathbf{x}) J_{R}^{a}(\mathbf{y})-2 J_{L}^{a}(\mathbf{x}) S_{A}^{a b}(\mathbf{z}) J_{R}^{b}(\mathbf{y})\right] \\
& +\int_{\mathbf{x}, \mathbf{y}, \mathbf{z}, \mathbf{z}^{\prime}} K_{\mathrm{JSSJ}}\left(\mathbf{x}, \mathbf{y}, \mathbf{z}, \mathbf{z}^{\prime}\right)\left[f^{a b c} f^{d e f} J_{L}^{a}(\mathbf{x}) S_{A}^{b e}(\mathbf{z}) S_{A}^{c f}\left(\mathbf{z}^{\prime}\right) J_{R}^{d}(\mathbf{y})-N_{c} J_{L}^{a}(\mathbf{x}) S_{A}^{a b}(\mathbf{z}) J_{R}^{b}(\mathbf{y})\right] \\
& +\int_{\mathbf{x}, \mathbf{y}, \mathbf{z}, \mathbf{z}^{\prime}} K_{q \bar{q}}\left(\mathbf{x}, \mathbf{y}, \mathbf{z}, \mathbf{z}^{\prime}\right)\left[2 J_{L}^{a}(\mathbf{x}) \operatorname{tr}\left[S^{\dagger}(\mathbf{z}) t^{a} S\left(\mathbf{z}^{\prime}\right) t^{b}\right] J_{R}^{b}(\mathbf{y})-J_{L}^{a}(\mathbf{x}) S_{A}^{a b}(\mathbf{z}) J_{R}^{b}(\mathbf{y})\right] \\
& +\int_{\mathbf{w}, \mathbf{x}, \mathbf{y}, \mathbf{z}, \mathbf{z}^{\prime}} K_{\mathrm{JJSSJ}}\left(\mathbf{w}, \mathbf{x}, \mathbf{y}, \mathbf{z}, \mathbf{z}^{\prime}\right) f^{a c b}\left[J_{L}^{d}(\mathbf{x}) J_{L}^{e}(\mathbf{y}) S_{A}^{d c}(\mathbf{z}) S_{A}^{e b}\left(\mathbf{z}^{\prime}\right) J_{R}^{a}(\mathbf{w})\right. \\
& \left.-J_{L}^{a}(\mathbf{w}) S_{A}^{c d}(\mathbf{z}) S_{A}^{b e}\left(\mathbf{z}^{\prime}\right) J_{R}^{d}(\mathbf{x}) J_{R}^{e}(\mathbf{y})+\frac{1}{3}\left(J_{L}^{c}(\mathbf{x}) J_{L}^{b}(\mathbf{y}) J_{L}^{a}(\mathbf{w})-J_{R}^{c}(\mathbf{x}) J_{R}^{b}(\mathbf{y}) J_{R}^{a}(\mathbf{w})\right)\right] \\
& +\int_{\mathbf{w}, \mathbf{x}, \mathbf{y}, \mathbf{z}} K_{\mathrm{JJSJ}}(\mathbf{w}, \mathbf{x}, \mathbf{y}, \mathbf{z}) f^{b d e}\left[J_{L}^{d}(\mathbf{x}) J_{L}^{e}(\mathbf{y}) S_{A}^{b a}(\mathbf{z}) J_{R}^{a}(\mathbf{w})\right. \\
& \left.-J_{L}^{a}(\mathbf{w}) S_{A}^{a b}(\mathbf{z}) J_{R}^{d}(\mathbf{x}) J_{R}^{e}(\mathbf{y})+\frac{1}{3}\left(J_{L}^{d}(\mathbf{x}) J_{L}^{e}(\mathbf{y}) J_{L}^{b}(\mathbf{w})-J_{R}^{d}(\mathbf{x}) J_{R}^{e}(\mathbf{y}) J_{R}^{b}(\mathbf{w})\right)\right]
\end{aligned}
$$


It is important to stress that in (2.56) all the rotation operators $J_{L}$ and $J_{R}$ are assumed not to act on $S$ in the Hamiltonian itself. We now list the kernels:

$$
\begin{aligned}
& K_{\mathrm{JJSSJ}}\left(\mathbf{w}, \mathbf{x}, \mathbf{y}, \mathbf{z}, \mathbf{z}^{\prime}\right)=-\frac{i \alpha_{s}^{2}}{4 \pi^{4}}\left(\frac{\left(Y^{\prime}\right)^{j} X^{i}}{\left(Y^{\prime}\right)^{2} X^{2}}-\frac{Y^{i}\left(X^{\prime}\right)^{j}}{\left(X^{\prime}\right)^{2} Y^{2}}\right) \\
& \quad \times\left(\frac{\delta^{i j}}{2 Z^{2}}-\frac{\left(W^{\prime}\right)^{j} Z^{i}}{\left(W^{\prime}\right)^{2} Z^{2}}+\frac{W^{i} Z^{j}}{W^{2} Z^{2}}-\frac{W^{i}\left(W^{\prime}\right)^{j}}{W^{2}\left(W^{\prime}\right)^{2}}\right) \ln \left(\frac{W^{2}}{\left(W^{\prime}\right)^{2}}\right), \\
& X \equiv \mathbf{x}-\mathbf{z}, X^{\prime} \equiv \mathbf{x}-\mathbf{z}^{\prime}, Y \equiv \mathbf{y}-\mathbf{z}, Y^{\prime} \equiv \mathbf{y}-\mathbf{z}^{\prime}, W \equiv \mathbf{w}-\mathbf{z}, W^{\prime} \equiv \mathbf{w}-\mathbf{z}^{\prime}, Z \equiv \mathbf{z}-\mathbf{z}^{\prime} . \\
& K_{\mathrm{JJSJ}}(\mathbf{w}, \mathbf{x}, \mathbf{y}, \mathbf{z})=-\frac{i \alpha_{s}^{2}}{4 \pi^{3}}\left(\frac{X \cdot W}{X^{2} W^{2}}-\frac{Y \cdot W}{Y^{2} W^{2}}\right) \ln \left(\frac{Y^{2}}{(X-Y)^{2}}\right) \ln \left(\frac{X^{2}}{(X-Y)^{2}}\right), \quad(2.58) \\
& K_{\mathrm{JSSJ}}\left(\mathbf{x}, \mathbf{y}, \mathbf{z}, \mathbf{z}^{\prime}\right)= \\
& \quad \frac{\alpha_{s}^{2}}{16 \pi^{4}}\left[\frac{4}{Z^{4}}+\left\{2 \frac{X^{2}\left(Y^{\prime}\right)^{2}+\left(X^{\prime}\right)^{2} Y^{2}-4(X-Y)^{2} Z^{2}}{Z^{4}\left(X^{2}\left(Y^{\prime}\right)^{2}-\left(X^{\prime}\right)^{2} Y^{2}\right)}+\frac{(X-Y)^{4}}{X^{2}\left(Y^{\prime}\right)^{2}-\left(X^{\prime}\right)^{2} Y^{2}}\right.\right. \\
& \left.\quad \times\left(\frac{1}{X^{2}\left(Y^{\prime}\right)^{2}}+\frac{1}{Y^{2}\left(X^{\prime}\right)^{2}}\right)+\frac{(X-Y)^{2}}{Z^{2}}\left(\frac{1}{X^{2}\left(Y^{\prime}\right)^{2}}-\frac{1}{Y^{2}\left(X^{\prime}\right)^{2}}\right)\right\} \ln \left(\frac{X^{2}\left(Y^{\prime}\right)^{2}}{\left(X^{\prime}\right)^{2} Y^{2}}\right) \\
& \left.\quad-\frac{2 I\left(\mathbf{x}, \mathbf{z}, \mathbf{z}^{\prime}\right)}{Z^{2}}-\frac{2 I\left(\mathbf{y}, \mathbf{z}, \mathbf{z}^{\prime}\right)}{Z^{2}}\right]+\widetilde{K}\left(\mathbf{x}, \mathbf{y}, \mathbf{z}, \mathbf{z}^{\prime}\right),
\end{aligned}
$$

where:

$$
\begin{gathered}
I\left(\mathbf{x}, \mathbf{z}, \mathbf{z}^{\prime}\right) \equiv \frac{1}{X^{2}-\left(X^{\prime}\right)^{2}}\left(\frac{X^{2}+\left(X^{\prime}\right)^{2}}{Z^{2}}-\frac{X \cdot X^{\prime}}{X^{2}}-\frac{X \cdot X^{\prime}}{\left(X^{\prime}\right)^{2}}-2\right) \ln \left(\frac{X^{2}}{\left(X^{\prime}\right)^{2}}\right) \\
\quad=\frac{1}{X^{2}-\left(X^{\prime}\right)^{2}}\left(\frac{X^{2}+\left(X^{\prime}\right)^{2}}{Z^{2}}+\frac{Z^{2}-X^{2}}{2\left(X^{\prime}\right)^{2}}+\frac{Z^{2}-\left(X^{\prime}\right)^{2}}{2 X^{2}}-3\right) \ln \left(\frac{X^{2}}{\left(X^{\prime}\right)^{2}}\right) .
\end{gathered}
$$

The terms which depend on one variable only, either $\mathbf{x}$ or $\mathbf{y}$, will not contribute when the Hamiltonian is taken to act on gauge invariant operators.

$$
\begin{aligned}
\widetilde{K}\left(\mathbf{x}, \mathbf{y}, \mathbf{z}, \mathbf{z}^{\prime}\right) \equiv & \frac{i}{2}\left(K_{\mathrm{JJSSJ}}\left(\mathbf{x}, \mathbf{x}, \mathbf{y}, \mathbf{z}, \mathbf{z}^{\prime}\right)-K_{\mathrm{JJSSJ}}\left(\mathbf{y}, \mathbf{x}, \mathbf{y}, \mathbf{z}, \mathbf{z}^{\prime}\right)\right. \\
& \left.-K_{\mathrm{JJSSJ}}\left(\mathbf{x}, \mathbf{y}, \mathbf{x}, \mathbf{z}, \mathbf{z}^{\prime}\right)+K_{\operatorname{JJSJ}}\left(\mathbf{y}, \mathbf{y}, \mathbf{x}, \mathbf{z}, \mathbf{z}^{\prime}\right)\right)
\end{aligned}
$$

Explicitly:

$$
\begin{gathered}
\widetilde{K}\left(\mathbf{x}, \mathbf{y}, \mathbf{z}, \mathbf{z}^{\prime}\right)=\frac{\alpha_{s}^{2}}{16 \pi^{4}}\left(\frac{\left(Y^{\prime}\right)^{2}}{\left(X^{\prime}\right)^{2} Z^{2} Y^{2}}-\frac{Y^{2}}{Z^{2} X^{2}\left(Y^{\prime}\right)^{2}}+\frac{1}{Z^{2}\left(Y^{\prime}\right)^{2}}-\frac{1}{Z^{2} Y^{2}}+\frac{(X-Y)^{2}}{X^{2} Z^{2} Y^{2}}\right. \\
\left.-\frac{(X-Y)^{2}}{\left(X^{\prime}\right)^{2} Z^{2}\left(Y^{\prime}\right)^{2}}+\frac{(X-Y)^{2}}{\left(X^{\prime}\right)^{2} X^{2}\left(Y^{\prime}\right)^{2}}-\frac{(X-Y)^{2}}{X^{2}\left(X^{\prime}\right)^{2} Y^{2}}\right) \ln \left(\frac{X^{2}}{\left(X^{\prime}\right)^{2}}\right)+(\mathbf{x} \leftrightarrow \mathbf{y}) .
\end{gathered}
$$

The following useful equality holds:

$$
\int_{\mathbf{z}^{\prime}} \widetilde{K}\left(\mathbf{x}, \mathbf{y}, \mathbf{z}, \mathbf{z}^{\prime}\right)=-\frac{\alpha_{s}^{2}}{4 \pi^{3}}\left(\frac{1}{X^{2}}-\frac{1}{Y^{2}}\right) \ln \left(\frac{Y^{2}}{(X-Y)^{2}}\right) \ln \left(\frac{X^{2}}{(X-Y)^{2}}\right)
$$


The kernel $K_{\text {JSJ }}$ reads: ${ }^{2}$

$$
\begin{aligned}
& K_{\mathrm{JSJ}}(\mathbf{x}, \mathbf{y}, \mathbf{z})=-\frac{\alpha_{s}^{2}}{16 \pi^{3}}\left(\frac { ( X - Y ) ^ { 2 } } { X ^ { 2 } Y ^ { 2 } } \left[b \ln \left((X-Y)^{2} \mu_{\overline{M S}}^{2}\right)-b \frac{X^{2}-Y^{2}}{(X-Y)^{2}} \ln \left(\frac{X^{2}}{Y^{2}}\right)\right.\right. \\
& \left.+2 b(\gamma-\ln 2)+\left(\frac{67}{9}-\frac{\pi^{2}}{3}\right) N_{c}-\frac{10}{9} N_{f}\right]-\left[\frac{1}{X^{2}}+\frac{1}{Y^{2}}\right]\left[2 b(\gamma-\ln 2)+\left(\frac{67}{9}-\frac{\pi^{2}}{3}\right) N_{c}\right. \\
& \left.\left.-\frac{10}{9} N_{f}\right]-\frac{b}{X^{2}} \ln X^{2} \mu_{\overline{M S}}^{2}-\frac{b}{Y^{2}} \ln Y^{2} \mu_{\overline{M S}}^{2}\right)-\frac{N_{c}}{2} \int_{\mathbf{z}^{\prime}} \widetilde{K}\left(\mathbf{x}, \mathbf{y}, \mathbf{z}, \mathbf{z}^{\prime}\right) .
\end{aligned}
$$

Here $\mu_{M S}^{2}$ is the normalisation point in the $\overline{M S}$ scheme and $b$ is the first coefficient of the $\beta$-function:

$$
b \equiv \frac{11 N_{c}-2 N_{f}}{3} .
$$

An alternative representation for $K_{\text {JSJ }}$ :

$$
\begin{aligned}
& K_{\mathrm{JSJ}}(\mathbf{x}, \mathbf{y}, \mathbf{z}) \\
& =\frac{\alpha_{s}^{2}}{8 \pi^{3}}\left[b\left(\frac{1}{2 X^{2}} \ln \left(Y^{2} \mu_{\overline{M S}}^{2}\right)+\frac{1}{2 Y^{2}} \ln \left(X^{2} \mu_{\overline{M S}}^{2}\right)-\frac{(X-Y)^{2}}{2 X^{2} Y^{2}} \ln \left((X-Y)^{2} \mu_{\overline{M S}}^{2}\right)\right)\right. \\
& \left.\quad+\frac{X \cdot Y}{X^{2} Y^{2}}\left[2 b(\gamma-\ln 2)+\left(\frac{67}{9}-\frac{\pi^{2}}{3}\right) N_{c}-\frac{10}{9} N_{f}\right]\right]-\frac{N_{c}}{2} \int_{\mathbf{z}^{\prime}} \widetilde{K}\left(\mathbf{x}, \mathbf{y}, \mathbf{z}, \mathbf{z}^{\prime}\right) .
\end{aligned}
$$

The quark sector:

$$
\begin{aligned}
K_{q \bar{q}}\left(\mathbf{x}, \mathbf{y}, \mathbf{z}, \mathbf{z}^{\prime}\right)= & \frac{\alpha_{s}^{2} N_{f}}{8 \pi^{4}}\left(\frac{2}{Z^{4}}-\frac{\left(X^{\prime}\right)^{2} Y^{2}+\left(Y^{\prime}\right)^{2} X^{2}-(X-Y)^{2} Z^{2}}{Z^{4}\left(X^{2}\left(Y^{\prime}\right)^{2}-\left(X^{\prime}\right)^{2} Y^{2}\right)} \ln \left(\frac{X^{2}\left(Y^{\prime}\right)^{2}}{\left(X^{\prime}\right)^{2} Y^{2}}\right)\right. \\
& \left.-\frac{I_{f}\left(\mathbf{x}, \mathbf{z}, \mathbf{z}^{\prime}\right)}{Z^{2}}-\frac{I_{f}\left(\mathbf{y}, \mathbf{z}, \mathbf{z}^{\prime}\right)}{Z^{2}}\right) \\
I_{f}\left(\mathbf{x}, \mathbf{z}, \mathbf{z}^{\prime}\right) \equiv & \frac{2}{Z^{2}}-\frac{2 X \cdot X^{\prime}}{Z^{2}\left(X^{2}-\left(X^{\prime}\right)^{2}\right)} \ln \left(\frac{X^{2}}{\left(X^{\prime}\right)^{2}}\right) .
\end{aligned}
$$

The kernels $K_{\mathrm{JJSSJ}}$ and $K_{\mathrm{JJSJ}}$ are anti-symmetric under $\mathbf{x}$ and $\mathbf{y}$ replacements, while the kernels $K_{\mathrm{JSJ}}, K_{\mathrm{JSSJ}}$, and $K_{q \bar{q}}$ are symmetric. Additional properties of the kernels and relations among them can be found in appendix D.

\section{The light cone wave function at NLO}

In this section, we compute the LCWF $|\psi\rangle$ perturbatively in $H_{\text {int }}$, up to order $g^{3}$. Normalisation of the LCWF is done to order $g^{4}$. Apart of the usual technical aspects, such as divergencies, related to loop calculations, there are two unfamiliar subtleties. The first one originates from the fact that the matrix elements of $H_{\mathrm{int}}(2.5)$ over the soft states defined in section 2.4 are non-commuting operators on the valence Hilbert space. We thus have to revise the standard perturbation theory in order to account for non-commutativity of the matrix elements, carefully tracing their ordering. This is done in appendix E. The second subtlety is related to the Born-Oppenheimer approximation and its applicability. It leads to a non-trivial condition on the phase of the LCWF, which otherwise cannot be determined from the perturbation theory. Summary of our results is presented in section 3.5.

\footnotetext{
${ }^{2}$ The term $2 b(\gamma-\ln 2)$ was missing in our previous results for $K_{\text {JSJ }}[57,59]$, even though it should be there as is clear from [92].
} 


\subsection{Third order perturbation theory}

General perturbative expression for the wave function before normalisation, correct up to order $g^{3}$, is computed in appendix E. After simplifying notations $\left(\left|n^{(i)}\right\rangle \longrightarrow|i\rangle\right)$ and also normalising the state when dividing by its norm:

$$
\begin{aligned}
\left|\psi^{\mathrm{NLO}}\right\rangle= & \mathcal{N}^{\mathrm{NLO}}|0\rangle-|i\rangle \frac{\left\langle i\left|H_{\mathrm{int}}\right| 0\right\rangle}{E_{i}}+|i\rangle \frac{\left\langle i\left|H_{\mathrm{int}}\right| j\right\rangle\left\langle j\left|H_{\mathrm{int}}\right| 0\right\rangle}{E_{i} E_{j}} \\
& -|i\rangle \frac{\left\langle i\left|H_{\mathrm{int}}\right| k\right\rangle\left\langle k\left|H_{\mathrm{int}}\right| j\right\rangle\left\langle j\left|H_{\mathrm{int}}\right| 0\right\rangle}{E_{i} E_{j} E_{k}}+|i\rangle \frac{\left|\left\langle j\left|H_{\mathrm{int}}\right| 0\right\rangle\right|^{2}\left\langle i\left|H_{\mathrm{int}}\right| 0\right\rangle\left(E_{i}+2 E_{j}\right)}{2 E_{i}^{2} E_{j}^{2}} .
\end{aligned}
$$

Compared to appendix E, the extra term $|i\rangle \frac{\left|\left\langle j\left|H_{\text {int }}\right| 0\right\rangle\right|^{2}\left\langle i\left|H_{\text {int }}\right| 0\right\rangle}{E_{i}^{2} E_{j}}$ is a contribution to the norm from the first order perturbation theory. Up to phase, $\mathcal{N}^{\mathrm{NLO}}$ is determined from normalisation of the state:

$$
\begin{aligned}
\left\|\mathcal{N}^{\mathrm{NLO}}\right\| \equiv & 1-\frac{\left|\left\langle i\left|H_{\mathrm{int}}\right| 0\right\rangle\right|^{2}}{2 E_{i}^{2}}+\frac{\left\langle 0\left|H_{\mathrm{int}}\right| i\right\rangle\left\langle i\left|H_{\mathrm{int}}\right| j\right\rangle\left\langle j\left|H_{\mathrm{int}}\right| 0\right\rangle}{2 E_{i} E_{j}}\left(\frac{1}{E_{i}}+\frac{1}{E_{j}}\right) \\
& -\frac{\left\langle 0\left|H_{\mathrm{int}}\right| i\right\rangle\left\langle i\left|H_{\mathrm{int}}\right| j\right\rangle\left\langle j\left|H_{\mathrm{int}}\right| k\right\rangle\left\langle k\left|H_{\mathrm{int}}\right| 0\right\rangle}{2 E_{i} E_{j} E_{k}}\left(\frac{1}{E_{i}}+\frac{1}{E_{j}}+\frac{1}{E_{k}}\right) \\
& +\frac{3\left|\left\langle j\left|H_{\mathrm{int}}\right| 0\right\rangle\right|^{2}\left|\left\langle i\left|H_{\mathrm{int}}\right| 0\right\rangle\right|^{2}}{8 E_{i}^{2} E_{j}^{2}} .
\end{aligned}
$$

Summation over repeated indices $i, j$ and $k$ is over the eigenstates of free Hamiltonian $H_{0}$, excluding the vacuum state $|0\rangle$. The ones that are relevant for the NLO calculation are defined in (2.20), (2.21), and (2.22). $E_{i}$ denotes the energy of the respective eigenstate, and $H_{\text {int }}$ is defined by (2.5). (3.1) is correct even when the matrix elements are operator valued and do not commute with each other. Notice that the overall phase of the wave function is not determined by the expansion (3.2). We will discuss below how this phase can be nevertheless uniquely fixed.

Our objective is to compute $\left|\psi^{\mathrm{NLO}}\right\rangle$. It is convenient to split the wave function according to its soft component content:

$$
\left|\psi^{\mathrm{NLO}}\right\rangle=\mathcal{N}^{\mathrm{NLO}}|0\rangle+\left|\psi_{g \rho}^{L O}\right\rangle+\sum_{i=1}^{i=2}\left|\psi_{q \bar{q}}^{i}\right\rangle+\sum_{i=1}^{i=3}\left|\psi_{g g}^{i}\right\rangle+\sum_{i=1}^{i=8}\left|\psi_{g}^{i}\right\rangle,
$$

where the subscripts $g, g g$ and $q \bar{q}$ correspond to the soft particle content in each state. $\left|\psi_{g \rho}^{L O}\right\rangle$ is the LO contribution (see section 2.7):

$\left|\psi_{g \rho}^{L O}\right\rangle=-\int_{\Lambda}^{e^{\delta \curlyvee} \Lambda} d k^{+} \int \frac{d^{2} \mathbf{k}}{(2 \pi)^{2}} \frac{g \mathbf{k}^{i} \rho^{a}(-\mathbf{k})}{\sqrt{\pi k^{+}} \mathbf{k}^{2}}\left|g_{i}^{a}(k)\right\rangle=\int_{\Lambda}^{e^{\delta \curlyvee} \Lambda} \frac{d k^{+}}{\sqrt{k^{+}}} \int_{\mathbf{x}, \mathbf{z}} \frac{i g \rho^{a}(\mathbf{x}) X^{i}}{2 \pi^{3 / 2} X^{2}}\left|g_{i}^{a}\left(k^{+}, \mathbf{z}\right)\right\rangle$.

The following states (integration over the momenta and summation over the quantum numbers of each particle are assumed) contribute and will be computed one by one below. Order $g^{2}$ states:

$$
\begin{aligned}
\left|\psi_{q \bar{q}}^{1}\right\rangle & \equiv|\bar{q} q\rangle\left\langle\bar{q} q\left|H_{g q q}\right| g\right\rangle\left\langle g\left|H_{g}\right| 0\right\rangle / E_{q \bar{q}} E_{g} \\
\left|\psi_{q \bar{q}}^{2}\right\rangle & \equiv-|\bar{q} q\rangle\left\langle\bar{q} q\left|H_{q q \text {-inst }}\right| 0\right\rangle / E_{q \bar{q}}
\end{aligned}
$$




$$
\begin{aligned}
\left|\psi_{g g}^{1}\right\rangle & \equiv|g g\rangle\left\langle g g\left|H_{g}\right| g\right\rangle\left\langle g\left|H_{g}\right| 0\right\rangle / E_{g g} E_{g}, \\
\left|\psi_{g g}^{2}\right\rangle & \equiv|g g\rangle\left\langle g g\left|H_{g g g}\right| g\right\rangle\left\langle g\left|H_{g}\right| 0\right\rangle / E_{g g} E_{g}, \\
\left|\psi_{g g}^{3}\right\rangle & \equiv-|g g\rangle\left\langle g g\left|H_{g g-\text { inst }}\right| 0\right\rangle / E_{g g} .
\end{aligned}
$$

Order $g^{3}$ states:

$$
\begin{aligned}
\left|\psi_{g}^{1}\right\rangle & \equiv-|g\rangle\left\langle g\left|H_{g q q}\right| \bar{q} q\right\rangle\left\langle\bar{q} q\left|H_{g q q}\right| g_{1}\right\rangle\left\langle g_{1}\left|H_{g}\right| 0\right\rangle / E_{g} E_{q \bar{q}} E_{g_{1}}, \\
\left|\psi_{g}^{2}\right\rangle & \equiv-|g\rangle\left\langle g\left|H_{g g g}\right| g g\right\rangle\left\langle g g\left|H_{g g g}\right| g_{1}\right\rangle\left\langle g_{1}\left|H_{g}\right| 0\right\rangle / E_{g} E_{g g} E_{g_{1}}, \\
\left|\psi_{g}^{3}\right\rangle & \equiv|g\rangle\left\langle g\left|H_{g g-\mathrm{inst}}\right| g_{1}\right\rangle\left\langle g_{1}\left|H_{g}\right| 0\right\rangle / E_{g} E_{g_{1}}, \\
\left|\psi_{g}^{4}\right\rangle & \equiv|g\rangle\left\langle g\left|H_{g}\right| g g\right\rangle\left\langle g g\left|H_{g g-\mathrm{inst}}\right| 0\right\rangle / E_{g} E_{g g}, \\
\left|\psi_{g}^{5}\right\rangle & \equiv-|g\rangle\left\langle g\left|H_{g}\right| g g\right\rangle\left\langle g g\left|H_{g}\right| g_{1}\right\rangle\left\langle g_{1}\left|H_{g}\right| 0\right\rangle / E_{g} E_{g g} E_{g_{1}}, \\
\left|\psi_{g}^{6}\right\rangle & \equiv-|g\rangle\left\langle g\left|H_{g g g}\right| g g\right\rangle\left\langle g g\left|H_{g}\right| g_{1}\right\rangle\left\langle g_{1}\left|H_{g}\right| 0\right\rangle / E_{g} E_{g g} E_{g_{1}}, \\
\left|\psi_{g}^{7}\right\rangle & \equiv-|g\rangle\left\langle g\left|H_{g}\right| g g\right\rangle\left\langle g g\left|H_{g g g}\right| g_{1}\right\rangle\left\langle g_{1}\left|H_{g}\right| 0\right\rangle / E_{g} E_{g g} E_{g_{1}}, \\
\left|\psi_{g}^{8}\right\rangle & \equiv|g\rangle\left\langle g\left|H_{g}\right| 0\right\rangle\left|\left\langle g_{1}\left|H_{g}\right| 0\right\rangle\right|^{2}\left(2 E_{g_{1}}+E_{g}\right) / 2 E_{g}^{2} E_{g_{1}}^{2} .
\end{aligned}
$$

The following contributions vanish:

$$
\begin{array}{r}
-|g\rangle\left\langle g\left|H_{g g g}\right| g g\right\rangle\left\langle g g\left|H_{g g-\text { inst }}\right| g_{1}\right\rangle\left\langle g_{1}\left|H_{g}\right| 0\right\rangle / E_{g} E_{g g} E_{g_{1}}=0, \\
-|g\rangle\left\langle g\left|H_{g g-\text { inst }}\right| g g\right\rangle\left\langle g g\left|H_{g g g}\right| g_{1}\right\rangle\left\langle g_{1}\left|H_{g}\right| 0\right\rangle / E_{g} E_{g g} E_{g_{1}}=0, \\
|g\rangle\left\langle g\left|H_{g g g g}\right| g_{1}\right\rangle\left\langle g_{1}\left|H_{g}\right| 0\right\rangle / E_{g} E_{\bar{g}_{1}}=0 .
\end{array}
$$

\subsection{Matrix elements}

We write down all the matrix elements that are relevant for the NLO calculation. These expressions are computed based on (2.29) - (2.34). At NLO there are two matrix elements for the soft-soft interactions and four matrix elements for the soft-valence interactions:

\section{- Gluon splits into quark and anti-quark pair.}

$$
\left\langle\bar{q}_{\lambda_{2}}^{\beta}(q) q_{\lambda_{1}}^{\alpha}(p)\left|H_{g q q}\right| g_{i}^{a}(k)\right\rangle=\frac{g t_{\alpha \beta}^{a}}{8 \pi^{3 / 2} \sqrt{k^{+}}} \chi_{\lambda_{1}}^{\dagger}\left[\frac{2 \mathbf{k}^{i}}{k^{+}}-\frac{\sigma \cdot \mathbf{p}}{p^{+}} \sigma^{i}-\sigma^{i} \frac{\sigma \cdot \mathbf{q}}{q^{+}}\right] \chi_{\lambda_{2}} \delta^{(3)}(k-p-q) \text {. }
$$

\section{- Triple gluon interaction.}

$$
\begin{gathered}
\left\langle g_{l}^{c}(q) g_{n}^{b}(p)\left|H_{g g g}\right| g_{m}^{a}(k)\right\rangle=\frac{i g f^{a b c} \delta^{(3)}(k-p-q)}{8 \pi^{3 / 2} \sqrt{k^{+} p^{+} q^{+}}}\left[\left(\mathbf{p}^{m}-\mathbf{q}^{m}+\frac{q^{+}-p^{+}}{k^{+}} \mathbf{k}^{m}\right) \delta_{n l}\right. \\
\left.+\left(\mathbf{k}^{n}+\mathbf{q}^{n}-\frac{k^{+}+q^{+}}{p^{+}} \mathbf{p}^{n}\right) \delta_{m l}+\left(\frac{k^{+}+p^{+}}{q^{+}} \mathbf{q}^{l}-\mathbf{p}^{l}-\mathbf{k}^{l}\right) \delta_{m n}\right] .
\end{gathered}
$$

- Valence adds an extra gluon to an existing one.

$$
\left\langle g_{l}^{c}(q) g_{j}^{b}(p)\left|H_{g}\right| g_{i}^{a}(k)\right\rangle=\delta^{a c} \delta_{i l} \delta^{(3)}(k-q) \frac{g \mathbf{p}^{j} \rho^{b}(-\mathbf{p})}{4 \pi^{3 / 2}\left|p^{+}\right|^{3 / 2}}+\delta^{a b} \delta_{i j} \delta^{(3)}(k-p) \frac{g \mathbf{q}^{l} \rho^{c}(-\mathbf{q})}{4 \pi^{3 / 2}\left|q^{+}\right|^{3 / 2}} .
$$


- Valence instantaneously creates a quark and anti-quark pair.

$$
\left\langle\bar{q}_{\lambda_{2}}^{\beta}(q) q_{\lambda_{1}}^{\alpha}(p)\left|H_{q q-\mathrm{inst}}\right| 0\right\rangle=\frac{g^{2} t_{\alpha \beta}^{a} \rho^{a}(-\mathbf{p}-\mathbf{q})}{8 \pi^{3}\left(p^{+}+q^{+}\right)^{2}} \chi_{\lambda_{1}}^{\dagger} \chi_{\lambda_{2}} \text {. }
$$

- Valence instantaneously creates two gluons.

$$
\left\langle g_{j}^{c}(q) g_{i}^{b}(p)\left|H_{g g-\text { inst }}\right| 0\right\rangle=\frac{i g^{2} f^{a b c}\left(q^{+}-p^{+}\right) \delta_{i j} \rho^{a}(-\mathbf{p}-\mathbf{q})}{2(2 \pi)^{3} \sqrt{p^{+} q^{+}}\left(p^{+}+q^{+}\right)^{2}} .
$$

- Valence instantaneously interacts with a soft gluon.

$$
\left\langle g_{j}^{c}(q)\left|H_{g g-\mathrm{inst}}\right| g_{i}^{b}(p)\right\rangle=\frac{i g^{2} f^{a b c}\left(p^{+}+q^{+}\right) \delta_{i j} \rho^{a}(\mathbf{p}-\mathbf{q})}{2(2 \pi)^{3} \sqrt{p^{+} q^{+}}\left(p^{+}-q^{+}\right)^{2}} .
$$

\subsection{Technical aspects of the calculation}

Before diving into the calculation of the LCWF (3.1) in the next subsection, let us first introduce several important technical aspect, which are used throughout this calculation.

- The decomposition procedure. As explained in the Introduction, the result of the calculation of the LCWF will have the form (1.2). Many of the terms we are to compute will have a product of more than one $\rho$ operator. These operators do not commute and their mutual ordering is very important. (3.1) will be later used to compute the NLO JIMWLK Hamiltonian. In order to compare with section 2.8, we would have to represent our result as in (2.56), including the symmetry properties for the kernels (the ones mentioned at the end of section 2.8). These symmetry properties do not come automatically in our calculation and we would have to manipulate our final results. It turns out that in order to achieve this, it suffices to symmetrise any product of two $\rho$ operators in the LCWF via the following identity:

$$
\begin{aligned}
\rho^{b}(-\mathbf{k}+\mathbf{p}) \rho^{a}(-\mathbf{p}) & =\frac{1}{2}\left[\rho^{b}(-\mathbf{k}+\mathbf{p}), \rho^{a}(-\mathbf{p})\right]+\frac{1}{2}\left\{\rho^{b}(-\mathbf{k}+\mathbf{p}), \rho^{a}(-\mathbf{p})\right\} \\
& =\frac{i}{2} f^{\text {bad }} \rho^{d}(-\mathbf{k})+\frac{1}{2}\left\{\rho^{b}(-\mathbf{k}+\mathbf{p}), \rho^{a}(-\mathbf{p})\right\}
\end{aligned}
$$

The replacement (3.27) will be referred to as "decomposition procedure". The physics behind this decomposition is that it explicitly separates between contributions from two emitters being in different transverse points in coordinate space from that when both emitters are in the same point. The latter is then equivalent to a contribution from a single emitter, in accordance to the colour algebra. After implementing the decomposition procedure where applicable, each component of the LCWF will receive an extra label in accord to the number of $\rho$ operators it contains. For example, in section 3.4.1 we show that $\left|\psi_{q \bar{q}}^{1}\right\rangle$ contains only one $\rho$ operator, and this component will be denoted thereafter by $\left|\psi_{q \bar{q} \rho}^{1}\right\rangle$. 
- Regularisation of divergent integrals. There are two types of divergencies that appear throughout the calculation. These are the usual divergencies in loop calculations. First, we will face divergencies that come from the longitudinal phase space integrals of the type $\int d k^{+} / k^{+}$, which correspond to emission of soft partons, just as in the case of LO calculation. These divergencies are regularised by confining the soft modes to live within the window $\Lambda<k^{+}<\Lambda e^{\delta Y}$. When computing the Hamiltonian in the next section, we will also encounter this longitudinal divergence "squared". Physically, such double divergence corresponds to independent (uncorrelated) emission of two soft gluons, which is accounted for by iteration of the LO Hamiltonian.

$\Lambda$ acts as an IR cutoff which will be set to zero (and $\delta Y$ to infinity) whenever it is safe to do so, without stating it explicitly.

UV divergences in transverse space integrals will be treated via dimensional regularisation, switching to $d \equiv 2-\epsilon$ dimensions,

$$
\int \frac{d^{2} \mathbf{p}}{(2 \pi)^{2}} \longrightarrow \mu^{\epsilon} \int \frac{d^{d} \mathbf{p}}{(2 \pi)^{d}}
$$

In addition, we will be frequently using the identity:

$$
\int d^{d} \mathbf{p} F\left(\mathbf{p}^{2}\right) \mathbf{p}^{i} \mathbf{p}^{j}=\frac{\delta^{i j}}{d} \int d^{d} \mathbf{p} F\left(\mathbf{p}^{2}\right) \mathbf{p}^{2}
$$

A couple of relevant integrals in dimensional regularisation are quoted in appendix C. As usual, (C.33) will be used for $\epsilon$ expansion. We will retain the divergent $\frac{1}{\epsilon}$ terms at the level of the wave function, as by itself the wave function is not a physical observable. Coupling constant renormalisation is done for physical observables, such as $\Sigma$ (or the JIMWLK Hamiltonian). This will be implemented in the $\overline{M S}$ scheme as a default action without any further explanations. The calculation also involves overlapping singularities when both the longitudinal and transverse integrals diverge. Those are treated similarly as above.

In addition to the above discussed UV divergencies, there are also potential IR divergencies at small transverse momenta. Those, however, do not emerge in our calculation explicitly because they always get regularised by the charge density operators $\rho$. Yet, this depends on a non-specified IR behaviour of the valence degrees of freedom and a priori is not guaranteed to lead to a safe IR. Particularly the IR divergencies resurface when the resulting JIMWLK Hamiltonian is taken to act on a gauge non-invariant operator, such as in the problem of gluon reggeization. The gluon trajectory is then found to be IR divergent even at LO.

- Phase of the LCWF. The perturbation theory cannot fix the phase of the LCWF, that is the phase of $\mathcal{N}^{\mathrm{NLO}}$. Normally, the phase is unobservable as it cancels in physical observables. However, this is not the case at hand, as we are not computing the full wave function but only its soft component. The normalisation coefficient $\mathcal{N}^{\mathrm{NLO}}$ is operator valued on the valence Hilbert space and thus its phase can make a non-trivial contribution to the observables. 
How can we find this phase which is clearly beyond the perturbation theory? We recall that our formalism is based on the Born-Oppenheimer adiabatic approximation. ${ }^{3}$ Obviously, there is some freedom in the phase, as, in principle, it can be always absorbed into the valence part of the LCWF. Yet, the factorisation (2.18) assumes that

$$
\left\langle v\left|\otimes\left\langle\psi\left|H_{V}\right| \psi\right\rangle \otimes\right| v\right\rangle \simeq\left\langle v\left|H_{V}\right| v\right\rangle \quad \text { or } \quad\left\langle\psi\left|H_{V}\right| \psi\right\rangle_{\text {soft }} \simeq 0 .
$$

where $H_{V}$ is a Hamiltonian for the valence degrees of freedom. The second expectation value is taken over the soft modes only. The condition (3.30) means that the dynamics of the soft modes does not significantly affect that of the valence. This assumption is implicit in the way the high energy factorisation is set, eliminating the freedom in the phase.

While the explicit form of $H_{V}$ is not known, the condition (3.30) presumably should be valid for any operator on the valence Hilbert space. Particularly, $H_{V}$ can be thought as been constructed from some powers of $\rho$, with (3.30) implying that they commute with $|\psi\rangle$ on the valence Hilbert space. Commuting one $\rho$ with $|\psi\rangle$ is equivalent to taking derivative $\delta / \delta \rho$. We thus require the following condition to hold

$$
\left\langle\psi\left|\frac{\delta}{\delta \rho^{d}(\mathbf{w})}\right| \psi\right\rangle=0 .
$$

Notice that (3.31) is the Berry connection, which we require to vanish. It is easy to check that at LO the condition is fulfilled by $\left|\psi^{L O}\right\rangle$ automatically. At NLO, this condition becomes a non-trivial constraint on the phase, which is worked out in appendix F.

\subsection{Computation of the NLO wave function}

In this section we compute the wave function (3.1). For each of the contributions (3.5)(3.17), a separate subsection is devoted. Lengthy computations are available in appendix G. The reader who is not interested in these details can proceed directly to the final results in section 3.5.

\subsubsection{Quark anti-quark state}

Computation of $\left|\boldsymbol{\psi}_{\boldsymbol{q} \bar{q}}^{\mathbf{1}}\right\rangle$. $\left|\psi_{q \bar{q} \rho}^{1}\right\rangle$ is defined in (3.5) (figure 4a).

$$
\begin{aligned}
\left|\psi_{q \bar{q}}^{1}\right\rangle \equiv & \sum_{\lambda_{1}, \lambda_{2}, f} \int_{\Lambda}^{e^{\delta \curlyvee} \Lambda} d k^{+} d p^{+} d q^{+} \int d^{2} \mathbf{k} d^{2} \mathbf{p} d^{2} \mathbf{q} \\
& \times\left|\bar{q}_{\lambda_{2}}^{\beta, f}(q) q_{\lambda_{1}}^{\alpha, f}(p)\right\rangle \frac{\left\langle\bar{q}_{\lambda_{2}}^{\beta, f}(q) q_{\lambda_{1}}^{\alpha, f}(p)\left|H_{g q q}\right| g_{i}^{a}(k)\right\rangle\left\langle g_{i}^{a}(k)\left|H_{g}\right| 0\right\rangle}{E_{q \bar{q}}(p, q) E_{g}(k)} .
\end{aligned}
$$

The physics content of (3.32) becomes clear by reading the matrix elements from the right to left: the first one corresponds to initial emission of a soft gluon from the valence current; then this soft gluon subsequently splits to a quark and anti-quark pair. The momentum conservation $\delta^{(3)}(q-k+p)$, which is explicit in the second matrix element (3.21), removes $q$ integral and effectively turns it into $k-p$. The process is illustrated in figure 4a.

\footnotetext{
${ }^{3}$ We would like to specially thank Alex Kovner who significantly helped us to establish the argument.
} 


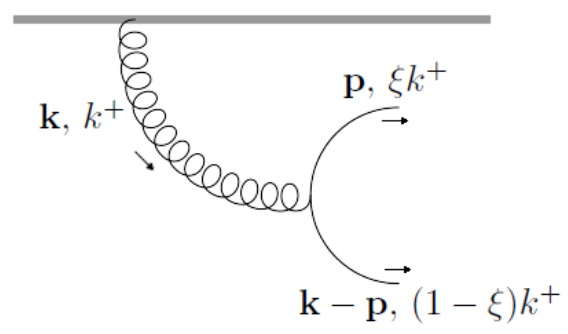

(a)

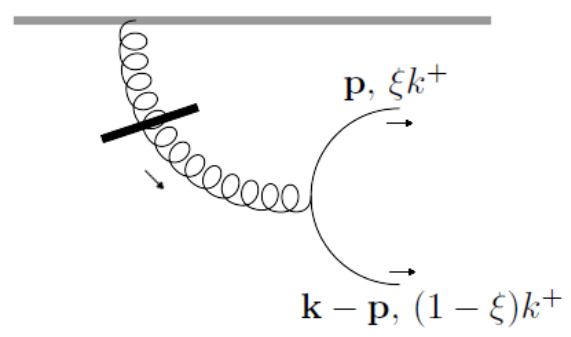

(b)

Figure 4. Quark and anti-quark emission diagrams. The bold black line over the gluon in $(b)$ marks the instantaneous interaction. $(a)-\left|\psi_{q \bar{q}}^{1}\right\rangle,(b)-\left|\psi_{q \bar{q}}^{2}\right\rangle$.

After inserting the relevant matrix elements, (2.49) and (3.21), and integrating over $q$ :

$$
\begin{aligned}
\left|\psi_{q \bar{q} \rho}^{1}\right\rangle \equiv & \left|\psi_{q \bar{q}}^{1}\right\rangle=\sum_{\lambda_{1}, \lambda_{2}, f} \int_{\Lambda}^{e^{\delta \curlyvee} \Lambda} d k^{+} \int_{0}^{1} d \xi \int d^{2} \mathbf{k} d^{2} \mathbf{p} \frac{4 \xi(1-\xi)}{\left((1-\xi) \mathbf{p}^{2}+\xi(\mathbf{k}-\mathbf{p})^{2}\right) \mathbf{k}^{2}} \\
& \times\left(\frac{g t_{\alpha \beta}^{a}}{8 \pi^{3 / 2}} \chi_{\lambda_{1}}^{\dagger}\left[2 \mathbf{k}^{i}-\frac{\sigma \cdot \mathbf{p}}{\xi} \sigma^{i}-\sigma^{i} \frac{\sigma \cdot(\mathbf{k}-\mathbf{p})}{1-\xi}\right] \chi_{\lambda_{2}}\right)\left(\frac{g \rho^{a}(-\mathbf{k}) \mathbf{k}^{i}}{4 \pi^{3 / 2}}\right) \\
& \times\left|\bar{q}_{\lambda_{2}}^{\beta, f}\left((1-\xi) k^{+}, \mathbf{k}-\mathbf{p}\right) q_{\lambda_{1}}^{\alpha, f}\left(\xi k^{+}, \mathbf{p}\right)\right\rangle .
\end{aligned}
$$

The variable $\xi$ is defined as:

$$
\xi \equiv \frac{p^{+}}{k^{+}}
$$

This definition will be used repeatedly throughout this paper.

Computation of $\left|\boldsymbol{\psi}_{\boldsymbol{q} \overline{\boldsymbol{q}}}^{\mathbf{2}}\right\rangle \cdot\left|\psi_{q \bar{q} \rho}^{2}\right\rangle$ is defined in (3.6) (figure $4 \mathrm{~b}$ ).

$$
\begin{aligned}
\left|\psi_{q \bar{q}}^{2}\right\rangle \equiv & -\sum_{\lambda_{1}, \lambda_{2}, f} \int_{\Lambda}^{e^{\delta \curlyvee} \Lambda} d k^{+} \int_{0}^{k^{+}} d p^{+} \int d^{2} \mathbf{k} d^{2} \mathbf{p} \\
& \times\left|\bar{q}_{\lambda_{2}}^{\beta, f}(k-p) q_{\lambda_{1}}^{\alpha, f}(p)\right\rangle \frac{\left\langle\bar{q}_{\lambda_{2}}^{\beta, f}(k-p) q_{\lambda_{1}}^{\alpha, f}(p)\left|H_{q q-\mathrm{inst}}\right| 0\right\rangle}{E_{q \bar{q}}(p, k-p)} .
\end{aligned}
$$

Inserting the matrix element (3.24) and changing variables according to (3.34):

$$
\begin{aligned}
\left|\psi_{q \bar{q} \rho}^{2}\right\rangle \equiv & \left|\psi_{q \bar{q}}^{2}\right\rangle=-\sum_{\lambda_{1}, \lambda_{2}, f} \int_{\Lambda}^{e^{\delta \curlyvee} \Lambda} d k^{+} \int_{0}^{1} d \xi \int d^{2} \mathbf{k} d^{2} \mathbf{p} \\
& \times \frac{g^{2} t_{\alpha \beta}^{a} \xi(1-\xi) \rho^{a}(-\mathbf{k}) \chi_{\lambda_{1}}^{\dagger} \chi_{\lambda_{2}}}{4 \pi^{3}\left((1-\xi) \mathbf{p}^{2}+\xi(\mathbf{k}-\mathbf{p})^{2}\right)}\left|\bar{q}_{\lambda_{2}}^{\beta, f}\left((1-\xi) k^{+}, \mathbf{k}-\mathbf{p}\right) q_{\lambda_{1}}^{\alpha, f}\left(\xi k^{+}, \mathbf{p}\right)\right\rangle .
\end{aligned}
$$

\subsubsection{Two gluon state}

Computation of $\left|\boldsymbol{\psi}_{\boldsymbol{g g}}^{\mathbf{1}}\right\rangle \cdot\left|\psi_{g g}^{1}\right\rangle$ is defined in (3.7) (figure 5a).

$$
\begin{aligned}
\left|\psi_{g g}^{1}\right\rangle \equiv & \frac{1}{2} \int_{\Lambda}^{e^{\delta \curlyvee} \Lambda} d q^{+} d k^{+} \int_{\Lambda}^{k^{+}-\Lambda} d p^{+} \int d^{2} \mathbf{q} d^{2} \mathbf{k} d^{2} \mathbf{p} \\
& \times\left|g_{j}^{b}(k-p) g_{l}^{c}(p)\right\rangle \frac{\left\langle g_{j}^{b}(k-p) g_{l}^{c}(p)\left|H_{g}\right| g_{i}^{a}(q)\right\rangle\left\langle g_{i}^{a}(q)\left|H_{g}\right| 0\right\rangle}{E_{g g}(p, k-p) E_{g}(q)} .
\end{aligned}
$$




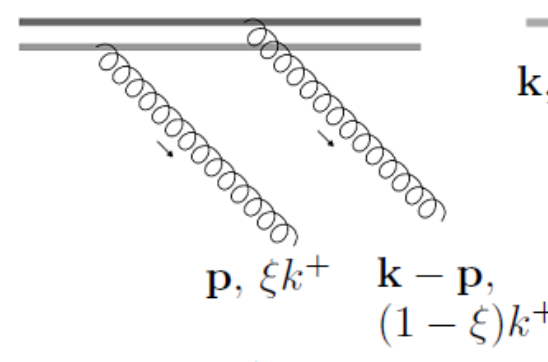

(a)

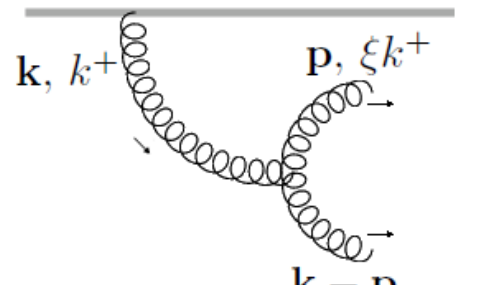

$\mathrm{k}-\mathrm{p}$,

$(1-\xi) k^{+}$

(b)

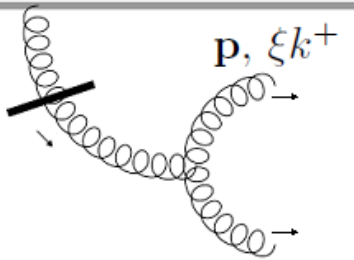

$\mathrm{k}-\mathrm{p}$,

$(1-\xi) k^{+}$

(c)

Figure 5. Two gluon producation diagrams. (a) - $\left|\psi_{g g}^{1}\right\rangle$, (b) - $\left|\psi_{g g}^{2}\right\rangle$, (c) - $\left|\psi_{g g}^{3}\right\rangle$.

Here $\frac{1}{2}$ is to avoid double-counting in two-gluon states. Inserting the matrix elements, (2.49) and (3.23):

$$
\begin{aligned}
\left|\psi_{g g}^{1}\right\rangle= & \frac{1}{2} \int_{\Lambda}^{e^{\delta \curlyvee} \Lambda} d k^{+} d q^{+} \int_{\Lambda}^{k^{+}-\Lambda} d p^{+} \int d^{2} \mathbf{k} d^{2} \mathbf{p} d^{2} \mathbf{q} \frac{1}{\frac{\mathbf{q}^{2}}{2 q^{+}}\left(\frac{\mathbf{p}^{2}}{2 p^{+}}+\frac{(\mathbf{k}-\mathbf{p})^{2}}{2\left(k^{+}-p^{+}\right)}\right)} \\
& \times\left(\delta^{a c} \delta_{i l} \delta^{(3)}(p-q) \frac{g\left(\mathbf{k}^{j}-\mathbf{p}^{j}\right) \rho^{b}(-\mathbf{k}+\mathbf{p})}{4 \pi^{3 / 2}\left|k^{+}-p^{+}\right|^{3 / 2}}+\delta^{a b} \delta_{j i} \delta^{(3)}(k-p-q) \frac{g \mathbf{p}^{l} \rho^{c}(-\mathbf{p})}{4 \pi^{3 / 2}\left|p^{+}\right|^{3 / 2}}\right) \\
& \times\left(\frac{g \mathbf{q}^{i} \rho^{a}(-\mathbf{q})}{4 \pi^{3 / 2}\left|q^{+}\right|^{3 / 2}}\right)\left|g_{j}^{b}(k-p) g_{l}^{c}(p)\right\rangle .
\end{aligned}
$$

The physics content of (3.37) is quite obvious and corresponds to subsequent emissions of two soft gluons. The momentum conservation imposed via the delta functions turns the momentum $q$ into either $k$ or $k-p$, which is illustrated in figure 5a. After integration over $q,(3.38)$ becomes:

$$
\begin{aligned}
\left|\psi_{g g}^{1}\right\rangle= & \int_{\Lambda}^{e^{\delta \curlyvee} \Lambda} d k^{+} \int d^{2} \mathbf{k} d^{2} \mathbf{p} \int_{\frac{\Lambda}{k^{+}}}^{1-\frac{\Lambda}{k^{+}}} d \xi \frac{g^{2} \mathbf{p}^{j}\left(\mathbf{k}^{i}-\mathbf{p}^{i}\right)}{8 \pi^{3}\left(\xi(\mathbf{k}-\mathbf{p})^{2}+(1-\xi) \mathbf{p}^{2}\right)} \\
& \times\left(\frac{\rho^{a}(-\mathbf{k}+\mathbf{p}) \rho^{b}(-\mathbf{p}) \sqrt{\xi}}{\mathbf{p}^{2} \sqrt{1-\xi}}+\frac{\rho^{b}(-\mathbf{p}) \rho^{a}(-\mathbf{k}+\mathbf{p}) \sqrt{1-\xi}}{(\mathbf{k}-\mathbf{p})^{2} \sqrt{\xi}}\right) \\
& \times\left|g_{i}^{a}\left((1-\xi) k^{+}, \mathbf{k}-\mathbf{p}\right) g_{j}^{b}\left(\xi k^{+}, \mathbf{p}\right)\right\rangle .
\end{aligned}
$$

Here $\xi \equiv \frac{p^{+}}{k^{+}}$as already defined in (3.34).

The result (3.39) can be decomposed according to (3.27), and represented as contributions with one and two $\rho$ operators:

$$
\left|\psi_{g g}^{1}\right\rangle \equiv\left|\psi_{g g \rho}^{1}\right\rangle+\left|\psi_{g g \rho \rho}\right\rangle,
$$

with

$$
\begin{aligned}
\left|\psi_{g g \rho}^{1}\right\rangle \equiv & \int_{\Lambda}^{e^{\delta \curlyvee} \Lambda} d k^{+} \int d^{2} \mathbf{k} d^{2} \mathbf{p} \int_{\frac{\Lambda}{k^{+}}}^{1-\frac{\Lambda}{k^{+}}} d \xi \frac{-i g^{2} f^{a b c} \mathbf{p}^{i}\left(\mathbf{k}^{j}-\mathbf{p}^{j}\right) \rho^{c}(-\mathbf{k}) \sqrt{\xi(1-\xi)}}{16 \pi^{3}\left(\xi(\mathbf{k}-\mathbf{p})^{2}+(1-\xi) \mathbf{p}^{2}\right)} \\
& \times\left(\frac{1}{(1-\xi) \mathbf{p}^{2}}-\frac{1}{\xi(\mathbf{k}-\mathbf{p})^{2}}\right)\left|g_{j}^{b}\left((1-\xi) k^{+}, \mathbf{k}-\mathbf{p}\right) g_{i}^{a}\left(\xi k^{+}, \mathbf{p}\right)\right\rangle
\end{aligned}
$$


and

$$
\begin{aligned}
\left|\psi_{g g \rho \rho}\right\rangle \equiv & \int_{\Lambda}^{e^{\delta \curlyvee} \Lambda} d k^{+} \int d^{2} \mathbf{k} d^{2} \mathbf{p} \int_{\frac{\Lambda}{k^{+}}}^{1-\frac{\Lambda}{k^{+}}} d \xi \\
& \times \frac{g^{2} \mathbf{p}^{i}\left(\mathbf{k}^{j}-\mathbf{p}^{j}\right)\left\{\rho^{a}(-\mathbf{p}), \rho^{b}(-\mathbf{k}+\mathbf{p})\right\}}{16 \pi^{3} \mathbf{p}^{2}(\mathbf{k}-\mathbf{p})^{2} \sqrt{\xi(1-\xi)}}\left|g_{j}^{b}\left((1-\xi) k^{+}, \mathbf{k}-\mathbf{p}\right) g_{i}^{a}\left(\xi k^{+}, \mathbf{p}\right)\right\rangle .
\end{aligned}
$$

Computation of $\left|\psi_{\boldsymbol{g g}}^{\mathbf{2}}\right\rangle \cdot\left|\psi_{\boldsymbol{g g}}^{2}\right\rangle$ is defined in (3.8) (figure 5b).

$$
\begin{aligned}
\left|\psi_{g g}^{2}\right\rangle \equiv & \frac{1}{2} \int_{\Lambda}^{e^{\delta \curlyvee} \Lambda} d k^{+} d p^{+} d q^{+} \int d^{2} \mathbf{k} d^{2} \mathbf{p} d^{2} \mathbf{q} \\
& \times\left|g_{j}^{b}(q) g_{l}^{c}(p)\right\rangle \frac{\left\langle g_{j}^{b}(q) g_{l}^{c}(p)\left|H_{g g g}\right| g_{i}^{a}(k)\right\rangle\left\langle g_{i}^{a}(k)\left|H_{g}\right| 0\right\rangle}{E_{g g}(p, q) E_{g}(k)} .
\end{aligned}
$$

The condition $q=k-p$ is enforced via momentum conservation, which is reflected on figure 5b. By inserting the relevant matrix elements, (2.49) and (3.22), and integrating over $q$ :

$$
\begin{aligned}
\left|\psi_{g g}^{2}\right\rangle= & \int_{\Lambda}^{e^{\delta \curlyvee} \Lambda} d k^{+} \int_{\Lambda}^{k^{+}} d p^{+} \int d^{2} \mathbf{k} d^{2} \mathbf{p} \frac{i g f^{a c b}}{16 \pi^{3 / 2}\left(\frac{\mathbf{p}^{2}}{2 p^{+}}+\frac{(\mathbf{k}-\mathbf{p})^{2}}{2\left(k^{+}-p^{+}\right)}\right)\left(\frac{\mathbf{k}^{2}}{2 k^{+}}\right) \sqrt{k^{+} p^{+}\left(k^{+}-p^{+}\right)}} \\
& \times\left(\left[2 \mathbf{p}^{i}-\frac{2 p^{+}}{k^{+}} \mathbf{k}^{i}\right] \delta_{j l}+\left[\frac{k^{+}+p^{+}}{k^{+}-p^{+}}\left(\mathbf{k}^{j}-\mathbf{p}^{j}\right)-\mathbf{k}^{j}-\mathbf{p}^{j}\right] \delta_{i l}+\left[2 \mathbf{k}^{l}-\frac{2 k^{+}}{p^{+}} \mathbf{p}^{l}\right] \delta_{i j}\right) \\
& \times\left(\frac{g \rho^{a}(-\mathbf{k}) \mathbf{k}^{i}}{4 \pi^{3 / 2}\left|k^{+}\right|^{3 / 2}}\right)\left|g_{j}^{b}(k-p) g_{l}^{c}(p)\right\rangle .
\end{aligned}
$$

By introducing new variables according to (3.34) and

$$
\mathbf{p}=\xi \mathbf{k}+\widetilde{\mathbf{p}}
$$

(3.44) becomes:

$$
\begin{aligned}
\left|\psi_{g g \rho}^{2}\right\rangle \equiv & \left|\psi_{g g}^{2}\right\rangle=-\int_{\Lambda}^{e^{\delta \curlyvee} \Lambda} d k^{+} \int d^{2} \mathbf{k} d^{2} \widetilde{\mathbf{p}} \int_{\frac{\Lambda}{k^{+}}}^{1-\frac{\Lambda}{k^{+}}} d \xi \frac{i g^{2} f^{a b c} \rho^{a}(-\mathbf{k}) \sqrt{\xi(1-\xi)} \mathbf{k}^{i}}{8 \pi^{3}\left(\mathbf{k}^{2} \xi(1-\xi)+\widetilde{\mathbf{p}}^{2}\right) \mathbf{k}^{2}} \\
& \times\left(\widetilde{\mathbf{p}}^{i} \delta_{j l}-\frac{1}{1-\xi} \widetilde{\mathbf{p}}^{j} \delta_{i l}-\frac{1}{\xi} \widetilde{\mathbf{p}}^{l} \delta_{i j}\right)\left|g_{j}^{b}\left((1-\xi) k^{+},(1-\xi) \mathbf{k}-\widetilde{\mathbf{p}}\right) g_{l}^{c}\left(\xi k^{+}, \xi \mathbf{k}+\widetilde{\mathbf{p}}\right)\right\rangle .
\end{aligned}
$$

Computation of $\left|\boldsymbol{\psi}_{\boldsymbol{g g}}^{\mathbf{3}}\right\rangle \cdot\left|\psi_{\mathrm{gg}}^{3}\right\rangle$ is defined in (3.9) (figure 5c.).

$$
\begin{aligned}
\left|\psi_{g g}^{3}\right\rangle \equiv & -\frac{1}{2} \int_{\Lambda}^{e^{\delta \curlyvee} \Lambda} d k^{+} \int_{\Lambda}^{k^{+}-\Lambda} d p^{+} \int d^{2} \mathbf{k} d^{2} \mathbf{p} \\
& \times\left|g_{i}^{b}(k-p) g_{j}^{c}(p)\right\rangle \frac{\left\langle g_{i}^{b}(k-p) g_{j}^{c}(p)\left|H_{g g-\text { inst }}\right| 0\right\rangle}{E_{g g}(k-p, p)} .
\end{aligned}
$$


After inserting the relevant matrix element (3.24) and changing variables according to (3.34) and (3.45), we can write the last result as:

$$
\begin{aligned}
\left|\psi_{g g \rho}^{3}\right\rangle \equiv\left|\psi_{g g}^{3}\right\rangle= & \int_{\Lambda}^{e^{\delta \curlyvee} \Lambda} d k^{+} \int d^{2} \mathbf{k} d^{2} \widetilde{\mathbf{p}} \int_{\frac{\Lambda}{k^{+}}}^{1-\frac{\Lambda}{k^{+}}} d \xi \frac{i g^{2} f^{a b c} \rho^{a}(-\mathbf{k})(1-2 \xi) \sqrt{\xi(1-\xi)}}{16 \pi^{3}\left(\mathbf{k}^{2} \xi(1-\xi)+\widetilde{\mathbf{p}}^{2}\right)} \delta_{i j} \\
& \times\left|g_{i}^{b}\left((1-\xi) k^{+},(1-\xi) \mathbf{k}-\widetilde{\mathbf{p}}\right) g_{j}^{c}\left(\xi k^{+}, \xi \mathbf{k}+\widetilde{\mathbf{p}}\right)\right\rangle
\end{aligned}
$$

While focusing on two-parton production, we have not encountered any divergencies. This is obviously because we have not yet ran into any loop integrations. At this stage we can only anticipate their appearance in the next section, where the overlaps of two wavefunctions are computed. Particularly, it is pretty obvious that $\psi_{q q}^{1}$ will lead to the logarithmic singularity in longitudinal momenta integration squared, which corresponds to second iteration of the LO JIMWLK Hamiltonian. Somewhat less obvious, but $\psi_{q q}^{3}$ also contributes to square of the LO. This is due to the kinematical region where one of the two gluons is much softer than another. In the next subsection, we will encounter both longitudinal and UV divergencies in the LCWF itself. The divergencies will be present in most of the loop corrections to the single gluon state.

\subsubsection{One gluon state}

Computation of $\left|\boldsymbol{\psi}_{\boldsymbol{g}}^{\mathbf{1}}\right\rangle \cdot \quad\left|\psi_{g}^{1}\right\rangle$ is defined in (3.10) (figure 6a.).

$$
\begin{aligned}
\left|\psi_{g}^{1}\right\rangle \equiv & -\int_{\Lambda}^{e^{\delta \curlyvee} \Lambda} d k^{+} d p^{+} d q^{+} d r^{+} \int d^{2} \mathbf{k} d^{2} \mathbf{p} d^{2} \mathbf{q} d^{2} \mathbf{r} \\
& \times\left|g_{j}^{d}(r)\right\rangle \frac{\left\langle g_{j}^{d}(r)\left|H_{g q q}\right| q_{\lambda_{1}}^{\alpha, f}(p) \bar{q}_{\lambda_{2}}^{\beta, f}(q)\right\rangle\left\langle q_{\lambda_{1}}^{\alpha, f}(p) \bar{q}_{\lambda_{2}}^{\beta, f}(q)\left|H_{g q q}\right| g_{i}^{a}(k)\right\rangle\left\langle g_{i}^{a}(k)\left|H_{g}\right| 0\right\rangle}{E_{g}(r) E_{q \bar{q}}(p, q) E_{g}(k)} .
\end{aligned}
$$

The physics of (3.49) is transparent from figure 6a. The figure reflects the momentum conservation, which eventually imposes $q=k-p$ and $r=k$. The rest of the calculation is available in appendix G.1. At the end of calculation there, we arrive at the following result:

$$
\begin{aligned}
\left|\psi_{g \rho}^{1}\right\rangle & \equiv\left|\psi_{g}^{1}\right\rangle \\
& =-\int_{\Lambda}^{e^{\delta \curlyvee} \Lambda} d k^{+} \int d^{2} \mathbf{k} \frac{g^{3} N_{f} \rho^{a}(-\mathbf{k}) \mathbf{k}^{i}}{32 \pi^{7 / 2} \sqrt{k^{+}} \mathbf{k}^{2}}\left(\frac{2}{3}\left[-\frac{2}{\epsilon}+\ln \left(\frac{\mathbf{k}^{2}}{\mu_{\overline{M S}}^{2}}\right)\right]-\frac{10}{9}\right)\left|g_{i}^{a}(k)\right\rangle,
\end{aligned}
$$

where we have introduced $\mu_{\overline{M S}}^{2} \equiv 4 \pi e^{-\gamma} \mu^{2} . \quad 2 / \epsilon$ reflects dimensional regularisation used to regulate the usual UV divergence of the quark loop integral. The renormalisation procedure will be applied in the next section, where we compute the physical Hamiltonian: the $2 / \epsilon$ singularity will be then absorbed into redefinition of the coupling constant. 


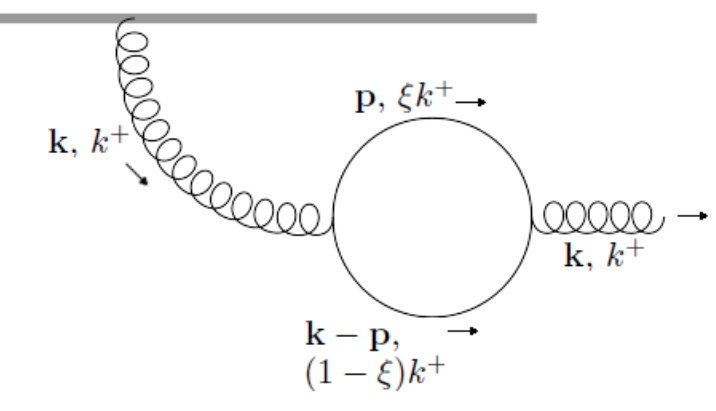

(a)

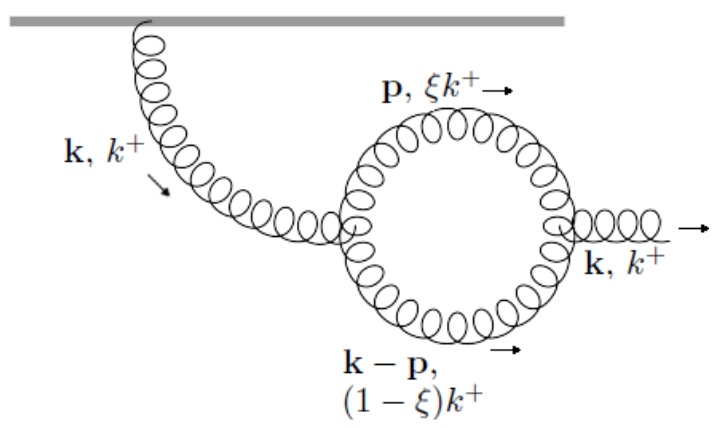

(b)

Figure 6. One gluon production. (a) - $\left|\psi_{g}^{1}\right\rangle$, (b) $-\left|\psi_{g}^{2}\right\rangle$.

Computation of $\left|\boldsymbol{\psi}_{\boldsymbol{g}}^{\mathbf{2}}\right\rangle \cdot \quad\left|\psi_{g}^{2}\right\rangle$ is defined in (3.11) (figure 6b).

$$
\begin{aligned}
\left|\psi_{g}^{2}\right\rangle \equiv & -\frac{1}{2} \int_{\Lambda}^{e^{\delta \curlyvee} \Lambda} d k^{+} d p^{+} d q^{+} d r^{+} \int d^{2} \mathbf{k} d^{2} \mathbf{p} d^{2} \mathbf{q} d^{2} \mathbf{r} \\
& \times\left|g_{l}^{d}(r)\right\rangle \frac{\left\langle g_{l}^{d}(r)\left|H_{g g g}\right| g_{k}^{c}(q) g_{j}^{b}(p)\right\rangle\left\langle g_{k}^{c}(q) g_{j}^{b}(p)\left|H_{g g g}\right| g_{i}^{a}(k)\right\rangle\left\langle g_{i}^{a}(k)\left|H_{g}\right| 0\right\rangle}{E_{g}(r) E_{g g}(p, q) E_{g}(k)},
\end{aligned}
$$

Just as in the previous calculation and as demonstrated on figure $6 \mathrm{~b}$, both $q$ and $r$ become $q=k-p$ and $r=k$ after realisation of the momentum conservation imposing delta functions. The rest of the calculation is available in appendix G.2. The result is:

$$
\begin{aligned}
\left|\psi_{g \rho}^{2}\right\rangle \equiv & \left|\psi_{g}^{2}\right\rangle=\int_{\Lambda}^{e^{\delta \curlyvee} \Lambda} d k^{+} \int d^{2} \mathbf{k} \frac{g^{3} N_{c} \rho^{a}(-\mathbf{k}) \mathbf{k}^{i}}{32 \pi^{7 / 2} \mathbf{k}^{2} \sqrt{k^{+}}} \\
& \times\left(\left[\frac{11}{3}+4 \ln \left(\frac{\Lambda}{k^{+}}\right)\right]\left[-\frac{2}{\epsilon}+\ln \left(\frac{\mathbf{k}^{2}}{\mu_{\overline{M S}}^{2}}\right)\right]+2 \ln ^{2}\left(\frac{\Lambda}{k^{+}}\right)-\frac{67}{9}+\frac{2 \pi^{2}}{3}\right)\left|g_{i}^{a}(k)\right\rangle .
\end{aligned}
$$

Apart the terms which are easily recognised as the gluon loop contribution to the $\beta$ function, we notice additional terms and particularly the $\ln \Lambda / k^{+}$, which are potentially divergent in the $\Lambda \rightarrow 0$ limit. These contributions arise from the phase space region where one of the gluons in the loop is much softer than another. This is precisely the kinematical domain where the triple gluon vertex $H_{g g g}$ admits eikonal approximation. It thus becomes clear that these logarithmic contributions correspond to iteration of the LO emission process. Indeed, in the next section we will demonstrate that all the $\ln \Lambda / k^{+}$ terms eventually contribute $\delta Y^{2}$ to the evolution identified with second iteration of the LO JIMWLK Hamiltonian.

Computation of $\left|\boldsymbol{\psi}_{\boldsymbol{g}}^{\mathbf{3}}\right\rangle \cdot\left|\psi_{g}^{3}\right\rangle$ is defined in (3.12).

$$
\left|\psi_{g}^{3}\right\rangle \equiv \int_{\Lambda}^{e^{\delta \curlyvee} \Lambda} d k^{+} \int_{\Lambda}^{e^{\delta \curlyvee} \Lambda} d p^{+} \int d^{2} \mathbf{k} d^{2} \mathbf{p}\left|g_{j}^{b}(k)\right\rangle \frac{\left\langle g_{j}^{b}(k)\left|H_{g g-\text { inst }}\right| g_{i}^{a}(p)\right\rangle\left\langle g_{i}^{a}(p)\left|H_{g}\right| 0\right\rangle}{E_{g}(k) E_{g}(p)} .
$$




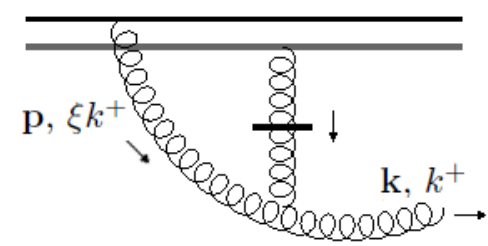

(a)

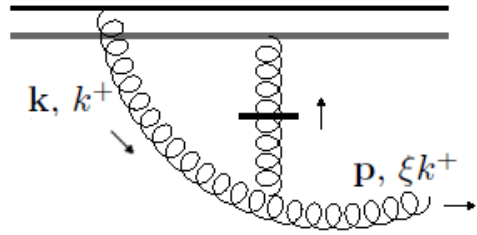

(b)

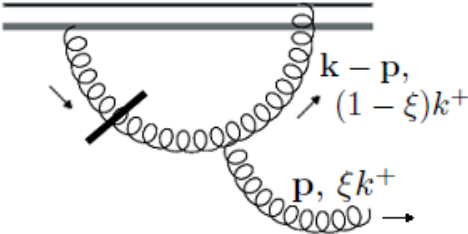

(c)

Figure 7. One gluon production via instantaneous interaction. (a) - $\left|\psi_{g}^{3 d}\right\rangle$, (b) - $\left|\psi_{g}^{3 u}\right\rangle$, (c) - $\left|\psi_{g}^{4}\right\rangle$.

Due to the instantaneous interaction, the gluon that was originally emitted can either gain or lose longitudinal momentum. The longitudinal integration over $p^{+}$in (3.53) can be split into two intervals:

$$
\int_{\Lambda}^{e^{\delta \curlyvee} \Lambda} d p^{+}=\int_{\Lambda}^{k^{+}-\Lambda} d p^{+}+\int_{k^{+}-\Lambda}^{e^{\delta \curlyvee} \Lambda} d p^{+}
$$

Accordingly, we also write $\left|\psi_{g}^{3}\right\rangle$ as a sum of two contributions $\left|\psi_{g}^{3 d}\right\rangle$ and $\left|\psi_{g}^{3 u}\right\rangle, \quad\left|\psi_{g}^{3}\right\rangle=$ $\left|\psi_{g}^{3 d}\right\rangle+\left|\psi_{g}^{3 u}\right\rangle$ where the upper script $u$ or $d$ represents the direction of the longitudinal momentum flow (corresponding to the integration intervals $\left[\Lambda, k^{+}-\Lambda\right]$ and $\left[k^{+}-\Lambda, e^{\delta \curlyvee} \Lambda\right]$ respectively). Let's start with $\left|\psi_{g}^{3 d}\right\rangle$ defined by

$$
\left|\psi_{g}^{3 d}\right\rangle \equiv \int_{\Lambda}^{e^{\delta \curlyvee} \Lambda} d k^{+} \int_{\Lambda}^{k^{+}-\Lambda} d p^{+} \int d^{2} \mathbf{k} d^{2} \mathbf{p}\left|g_{j}^{b}(k)\right\rangle \frac{\left\langle g_{j}^{b}(k)\left|H_{g g-\mathrm{inst}}\right| g_{i}^{a}(p)\right\rangle\left\langle g_{i}^{a}(p)\left|H_{g}\right| 0\right\rangle}{E_{g}(k) E_{g}(p)}
$$

and illustrated in figure $7 \mathrm{a}$, where our standard relation $p^{+}=\xi k^{+}$is also used. The rest of the calculation is available in appendix G.3. The end result integrated over $\xi$ with the aid of (C.7) reads:

$$
\begin{aligned}
\left|\psi_{g \rho \rho}^{3 d}\right\rangle \equiv & \left|\psi_{g}^{3 d}\right\rangle=\int_{\Lambda}^{e^{\delta \curlyvee} \Lambda} d k^{+} \int d^{2} \mathbf{k} d^{2} \mathbf{p} \frac{i g^{3} f^{a b c} \mathbf{p}^{i}\left\{\rho^{a}(-\mathbf{p}), \rho^{c}(-\mathbf{k}+\mathbf{p})\right\}}{16 \pi^{9 / 2} \mathbf{k}^{2} \mathbf{p}^{2} \sqrt{k^{+}}} \\
& \times\left(\frac{k^{+}}{\Lambda}-1-\ln \left(\frac{\Lambda}{k^{+}}\right)\right)\left|g_{i}^{b}(k)\right\rangle .
\end{aligned}
$$

We seem to again encounter the $\ln \left(k^{+} / \Lambda\right)$ type divergence. The latter is, however, fictitious and does not represent any physics. The divergence will cancel when we collect all similar contributions.

Next is $\left|\psi_{g}^{3 u}\right\rangle$. We find it convenient to exchange the dummy momenta $k$ and $p, k \leftrightarrow p$. The expression for $\left|\psi_{g}^{3 u}\right\rangle$ is then given by

$$
\left|\psi_{g}^{3 u}\right\rangle \equiv \int_{\Lambda}^{e^{\delta \curlyvee} \Lambda} d k^{+} \int_{\Lambda}^{k^{+}-\Lambda} d p^{+} \int d^{2} \mathbf{k} d^{2} \mathbf{p}\left|g_{j}^{b}(p)\right\rangle \frac{\left\langle g_{j}^{b}(p)\left|H_{g g-\mathrm{inst}}\right| g_{i}^{a}(k)\right\rangle\left\langle g_{i}^{a}(k)\left|H_{g}\right| 0\right\rangle}{E_{g}(p) E_{g}(k)} .
$$


and illustrated in figure $7 \mathrm{~b}$. The result obtained after inserting the relevant matrix elements, (2.49) and (3.26), and changing variables according to (3.34) becomes:

$$
\begin{aligned}
\left|\psi_{g}^{3 u}\right\rangle= & \int_{\Lambda}^{e^{\delta \curlyvee} \Lambda} d k^{+} \int_{\Lambda}^{k^{+}-\Lambda} d p^{+} \int d^{2} \mathbf{k} d^{2} \mathbf{p} \frac{1}{\left(\frac{\mathbf{p}^{2}}{2 p^{+}}\right)\left(\frac{\mathbf{k}^{2}}{2 k^{+}}\right)} \\
& \times\left(\frac{i g^{2} f^{a b c}\left(p^{+}+k^{+}\right) \rho^{c}(-\mathbf{p}+\mathbf{k}) \delta_{j}^{i}}{2(2 \pi)^{3} \sqrt{k^{+} p^{+}}\left(k^{+}-p^{+}\right)^{2}}\right)\left(\frac{g \rho^{a}(-\mathbf{k}) \mathbf{k}^{i}}{4 \pi^{3 / 2}\left|k^{+}\right|^{3 / 2}}\right)\left|g_{j}^{b}(p)\right\rangle .
\end{aligned}
$$

Introducing $\xi$ for compactness the last result is:

$$
\begin{aligned}
\left|\psi_{g \rho \rho}^{3 u}\right\rangle \equiv & \left|\psi_{g}^{3 u}\right\rangle=\int_{\Lambda}^{e^{\delta \curlyvee} \Lambda} d k^{+} \int d^{2} \mathbf{k} d^{2} \mathbf{p} \int_{\frac{\Lambda}{k^{+}}}^{1-\frac{\Lambda}{k^{+}}} d \xi \\
& \times \frac{i g^{3} f^{a b c}(1+\xi) \sqrt{\xi}\left\{\rho^{c}(-\mathbf{k}+\mathbf{p}), \rho^{a}(-\mathbf{p})\right\} \mathbf{p}^{i}}{32 \pi^{9 / 2}(1-\xi)^{2} \mathbf{k}^{2} \mathbf{p}^{2} \sqrt{k^{+}}}\left|g_{i}^{b}\left(\xi k^{+}, \mathbf{k}\right)\right\rangle .
\end{aligned}
$$

In principle, it is possible to compute one of the longitudinal integrals in (3.59), but we have chosen to postpone this step until the final result gets assembled.

Computation of $\left|\boldsymbol{\psi}_{\boldsymbol{g}}^{\mathbf{4}}\right\rangle$ and $\left|\boldsymbol{\psi}_{\boldsymbol{g}}^{\mathbf{5}}\right\rangle$. The contributions $\left|\psi_{g}^{4}\right\rangle$ and $\left|\psi_{g}^{5}\right\rangle$ have very similar integrand structures. This is the reason we founnd it convenient to combine the two. $\left|\psi_{g}^{4}\right\rangle$ is defined in (3.13) (figure 7c),

$$
\begin{aligned}
\left|\psi_{g}^{4}\right\rangle \equiv & \frac{1}{2} \int_{\Lambda}^{e^{\delta \curlyvee} \Lambda} d q^{+} d k^{+} \int_{\Lambda}^{k^{+}-\Lambda} d p^{+} \int d^{2} \mathbf{q} d^{2} \mathbf{k} d^{2} \mathbf{p} \\
& \times\left|g_{l}^{d}(p)\right\rangle \frac{\left\langle g_{l}^{d}(p)\left|H_{g}\right| g_{j}^{b}(q) g_{i}^{a}(k-q)\right\rangle\left\langle g_{j}^{b}(q) g_{i}^{a}(k-q)\left|H_{g g-\text { inst }}\right| 0\right\rangle}{E_{g}(p) E_{g g}(q, k-q)} .
\end{aligned}
$$

The matrix element $\left\langle g_{l}^{d}(p)\left|H_{g}\right| g_{j}^{b}(q) g_{i}^{a}(k-q)\right\rangle$ contains a momentum conserving delta function which eventually sets $q=p$ (or $q=k-p$ ). Figure 7c illustrates the process. $\left|\psi_{g}^{4}\right\rangle$ is computed in appendix G.4. $\left|\psi_{g}^{5}\right\rangle$ is defined in (3.14) (figure 8a),

$$
\begin{aligned}
\left|\psi_{g}^{5}\right\rangle \equiv & -\frac{1}{2} \int_{\Lambda}^{e^{\delta \curlyvee} \Lambda} d k^{+} d p^{+} d q^{+} d r^{+} \int d^{2} \mathbf{k} d^{2} \mathbf{p} d^{2} \mathbf{q} d^{2} \mathbf{r} \\
& \times\left|g_{m}^{d}(p)\right\rangle \frac{\left\langle g_{m}^{d}(p)\left|H_{g}\right| g_{k}^{c}(r) g_{j}^{b}(q)\right\rangle\left\langle g_{k}^{c}(r) g_{j}^{b}(q)\left|H_{g g g}\right| g_{i}^{a}(k)\right\rangle\left\langle g_{i}^{a}(k)\left|H_{g}\right| 0\right\rangle}{E_{g}(k) E_{g g}(q, r) E_{g}(p)} .
\end{aligned}
$$

The momentum conservation in $\left|\psi_{g}^{5}\right\rangle$ imposes $q=p$ and $r=k-p$ (or $q=k-p$ and $r=p$ ), which is the case displayed in figure 8a. The calculation of $\left|\psi_{g}^{5}\right\rangle$ can be found in appendix G.4.

We now introduce sum of $\left|\psi_{g}^{4}\right\rangle$ and $\left|\psi_{g}^{5}\right\rangle$,

$$
\left|\psi_{g}^{4+5}\right\rangle \equiv\left|\psi_{g}^{5}\right\rangle+\left|\psi_{g}^{4}\right\rangle .
$$

Next, $\left|\psi_{g}^{4+5}\right\rangle$ is split according to (3.27) into two parts involving one and two $\rho$ operators:

$$
\left|\psi_{g}^{4+5}\right\rangle=\left|\psi_{g \rho}^{4+5}\right\rangle+\left|\psi_{g \rho \rho}^{4+5}\right\rangle .
$$

All the algebra is performed in appendix G.4. Below, we quote the final results only. 


\section{- One $\rho$ part.}

$$
\begin{aligned}
& \left|\psi_{g \rho}^{4+5}\right\rangle \equiv \int_{\Lambda}^{e^{\delta \curlyvee} \Lambda} d k^{+} \int d^{2} \mathbf{k} \int_{\frac{\Lambda}{k^{+}}}^{1-\frac{\Lambda}{k^{+}}} d \xi \frac{g^{3} N_{c} \rho^{a}(-\mathbf{k}) \mathbf{k}^{j}}{32 \pi^{7 / 2} \sqrt{\xi}(1-\xi) \mathbf{k}^{2} \sqrt{k^{+}}} \\
& \times\left((\xi-2) \ln (\xi)+(\xi+1) \ln (1-\xi)+(\xi-2)\left[-\frac{2}{\epsilon}+\ln \left(\frac{\mathbf{k}^{2}}{\mu_{\overline{M S}}^{2}}\right)\right]\right)\left|g_{j}^{a}\left(\xi k^{+}, \mathbf{k}\right)\right\rangle .
\end{aligned}
$$

One of the longitudinal integrals, either $\xi$ or $k^{+}$, in (3.64) could be computed, but we have again chosen to leave the expressions as is until the final result is assembled. Yet, we see that the longitudinal integral would result in a logarithmic divergence, although as usual regularised by $\Lambda$. The origin of this divergence can be traced to $\left|\psi_{g}^{5}\right\rangle$ and particularly to the phase space region where $H_{g g g}$ admits eikonal approximation. Thus $\left|\psi_{g}^{5}\right\rangle$ has a piece, which can be interpreted as a soft gluon emission from a LO evolved LCWF. In the next section, such contributions will be identified with $(\mathrm{LO})^{2}$ terms.

Furthermore, $2 / \epsilon$ points to a regularised UV divergence. Several diagrams below will also contribute such terms. Most, however, will cancel against each other. Some UV divergence will nevertheless survive and that is a feature of the LCWF at NLO. It will not, however, contribute any UV divergence at the level of the Hamiltonian. There they will cancel against a subtraction term made of two produced gluons, which cross the shock wave at the same transverse point.

- Two $\boldsymbol{\rho}$ part. From (G.26) (after the substitution $\mathbf{p} \leftrightarrow \mathbf{k}$ ):

$$
\begin{aligned}
& \left|\psi_{g \rho \rho}^{4+5}\right\rangle \equiv \int_{\Lambda}^{e^{\delta \curlyvee} \Lambda} d k^{+} \int d^{2} \mathbf{k} d^{2} \mathbf{p} \int_{\frac{\Lambda}{k^{+}}}^{1-\frac{\Lambda}{k^{+}}} d \xi \frac{i g^{3} f^{a b c}\left\{\rho^{c}(-\mathbf{k}+\mathbf{p}), \rho^{a}(-\mathbf{p})\right\} \xi^{3 / 2}}{32 \pi^{9 / 2} \mathbf{k}^{2} \mathbf{p}^{2}\left(\xi(\mathbf{k}-\mathbf{p})^{2}+(1-\xi) \mathbf{k}^{2}\right)(1-\xi) \sqrt{k^{+}}} \\
& \times\left(\left[\frac{2}{\xi}\left(\mathbf{p}^{2}-\mathbf{k} \cdot \mathbf{p}\right)-\mathbf{p}^{2}+2 \mathbf{k} \cdot \mathbf{p}\right] \mathbf{k}^{j}-\left[\mathbf{k}^{2}+\frac{1+\xi}{1-\xi}(\mathbf{k}-\mathbf{p})^{2}\right] \mathbf{p}^{j}\right)\left|g_{j}^{b}\left(\xi k^{+}, \mathbf{k}\right)\right\rangle \cdot
\end{aligned}
$$

A convenient way to represent (3.65):

$$
\begin{aligned}
\left|\psi_{g \rho \rho}^{4+5}\right\rangle= & -\left|\psi_{g \rho \rho}^{3 u}\right\rangle \\
& +\int_{\Lambda}^{e^{\delta \curlyvee} \Lambda} d k^{+} \int d^{2} \mathbf{k} d^{2} \mathbf{p} \int_{\frac{\Lambda}{k^{+}}}^{1-\frac{\Lambda}{k^{+}}} d \xi \frac{i g^{3} f^{a b c}\left\{\rho^{c}(-\mathbf{k}+\mathbf{p}), \rho^{a}(-\mathbf{p})\right\} \xi^{3 / 2}}{32 \pi^{9 / 2} \mathbf{k}^{2} \mathbf{p}^{2}\left(\xi(\mathbf{k}-\mathbf{p})^{2}+(1-\xi) \mathbf{k}^{2}\right)(1-\xi) \sqrt{k^{+}}} \\
& \times\left(\left[\frac{2}{\xi}\left(\mathbf{p}^{2}-\mathbf{k} \cdot \mathbf{p}\right)-\mathbf{p}^{2}+2 \mathbf{k} \cdot \mathbf{p}\right] \mathbf{k}^{i}+\frac{1}{\xi} \mathbf{k}^{2} \mathbf{p}^{i}\right)\left|g_{i}^{b}\left(\xi k^{+}, \mathbf{k}\right)\right\rangle .
\end{aligned}
$$

The advantage of the last representation is obvious: in the next subsection we will add up $\left|\psi_{g \rho \rho}^{4+5}\right\rangle$ with $\left|\psi_{g \rho \rho}^{3 u}\right\rangle$ and the mutual cancelation becomes transparent via representation (3.66).

Computation of $\left|\boldsymbol{\psi}_{\boldsymbol{g}}^{\mathbf{6}}\right\rangle \cdot \quad\left|\psi_{g}^{6}\right\rangle$ is defined in (3.15) (figure 8b).

$$
\begin{aligned}
\left|\psi_{g}^{6}\right\rangle \equiv & -\frac{1}{2} \int_{\Lambda}^{e^{\delta \curlyvee} \Lambda} d k^{+} d p^{+} d q^{+} d r^{+} \int d^{2} \mathbf{k} d^{2} \mathbf{p} d^{2} \mathbf{q} d^{2} \mathbf{r} \\
& \times\left|g_{l}^{e}(k)\right\rangle \frac{\left\langle g_{l}^{e}(k)\left|H_{g g g}\right| g_{n}^{c}(r) g_{j}^{b}(p)\right\rangle\left\langle g_{n}^{c}(r) g_{j}^{b}(p)\left|H_{g}\right| g_{i}^{a}(q)\right\rangle\left\langle g_{i}^{a}(q)\left|H_{g}\right| 0\right\rangle}{E_{g}(k) E_{g g}(p, r) E_{g}(q)} .
\end{aligned}
$$




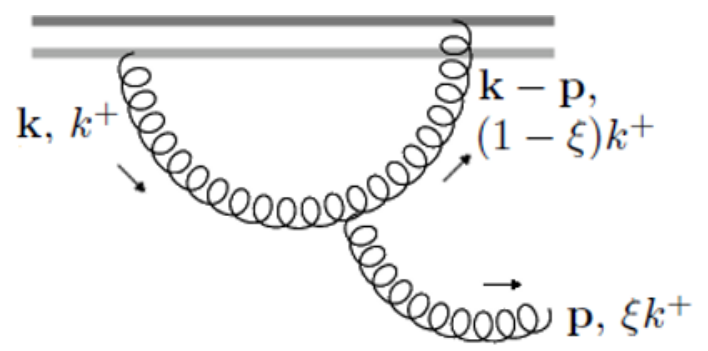

(a)

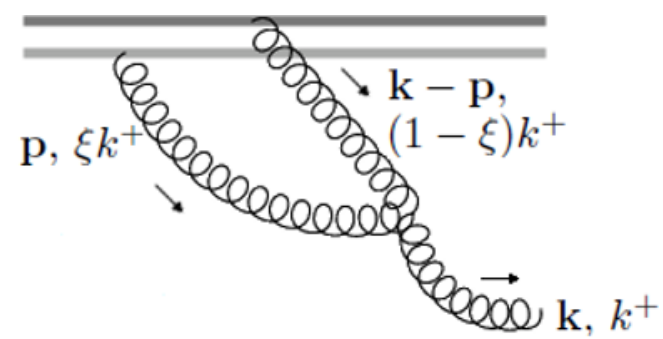

(b)

Figure 8. One gluon production diagrams involving the triple gluon vertex. (a) - $\left|\psi_{g}^{5}\right\rangle$, (b) - $\left|\psi_{g}^{6}\right\rangle$.

Just as in the previous calculations, the momentum conservation imposes $q=k$ and $r=k-p$. This is how this process is illustrated in figure $8 \mathrm{~b}$ with our usual definition of $\xi$. The rest of the calculation is available in appendix G.5, from which we quote:

$$
\begin{aligned}
\left|\psi_{g}^{6}\right\rangle & =-\int_{\Lambda}^{e^{\delta \curlyvee} \Lambda} d k^{+} \int d^{2} \mathbf{k} d^{2} \mathbf{p} \int_{\frac{\Lambda}{k^{+}}}^{1-\frac{\Lambda}{k^{+}}} d \xi \frac{i g^{3} f^{\mathrm{bad}} \rho^{b}(-\mathbf{p}) \rho^{a}(-\mathbf{k}+\mathbf{p})}{16 \pi^{9 / 2} \xi(\mathbf{k}-\mathbf{p})^{2}\left((1-\xi) \mathbf{p}^{2}+\xi(\mathbf{k}-\mathbf{p})^{2}\right) \mathbf{k}^{2} \sqrt{k^{+}}} \\
\times & {\left[\left(-2(1-\xi) \mathbf{k} \cdot \mathbf{p}+\frac{2\left(1-\xi^{2}\right)}{\xi} \mathbf{p}^{2}\right) \mathbf{k}^{l}+\left(\mathbf{k}^{2}-\frac{1+\xi}{1-\xi}(\mathbf{k}-\mathbf{p})^{2}+\frac{\xi-2}{\xi} \mathbf{p}^{2}\right) \mathbf{p}^{l}\right]\left|g_{l}^{d}(k)\right\rangle . }
\end{aligned}
$$

The contributions with one and two $\rho$ operators can be isolated according to (3.27):

$$
\left|\psi_{g}^{6}\right\rangle=\left|\psi_{g \rho}^{6}\right\rangle+\left|\psi_{g \rho \rho}^{6}\right\rangle .
$$

- One $\rho$ part.

$$
\begin{aligned}
\left|\psi_{g \rho}^{6}\right\rangle \equiv & -\int_{\Lambda}^{e^{\delta \curlyvee} \Lambda} d k^{+} \int d^{2} \mathbf{k} \frac{g^{3} N_{c} \rho^{a}(-\mathbf{k}) \mathbf{k}^{i}}{32 \pi^{7 / 2} \mathbf{k}^{2} \sqrt{k^{+}}} \\
& \times\left(3 \ln \left(\frac{\Lambda}{k^{+}}\right)\left[-\frac{2}{\epsilon}+\ln \left(\frac{\mathbf{k}^{2}}{\mu^{2}}\right)\right]+\frac{\pi^{2}}{3}+2 \ln ^{2}\left(\frac{\Lambda}{k^{+}}\right)\right)\left|g_{i}^{a}(k)\right\rangle .
\end{aligned}
$$

- Two $\rho$ part.

$$
\begin{aligned}
\left|\psi_{g \rho \rho}^{6}\right\rangle \equiv & -\int_{\Lambda}^{e^{\delta \curlyvee} \Lambda} d k^{+} \int d^{2} \mathbf{k} d^{2} \mathbf{p} \frac{i g^{3} f^{a b c}\left\{\rho^{b}(-\mathbf{p}), \rho^{c}(-\mathbf{k}+\mathbf{p})\right\}}{32 \pi^{9 / 2} \mathbf{p}^{2}(\mathbf{k}-\mathbf{p})^{2} \mathbf{k}^{2} \sqrt{k^{+}}}\left[\ln \left(\frac{\Lambda}{k^{+}}\right) \mathbf{k} \cdot \mathbf{p k}^{l}\right. \\
& \left.-\left(\ln \left(\frac{\Lambda}{k^{+}}\right) \mathbf{k}^{2}+2\left(\frac{k^{+}}{\Lambda}-1-\ln \left(\frac{\Lambda}{k^{+}}\right)\right)(\mathbf{k}-\mathbf{p})^{2}\right) \mathbf{p}^{l}\right]\left|g_{l}^{a}(k)\right\rangle .
\end{aligned}
$$

Computation of $\left|\boldsymbol{\psi}_{\boldsymbol{g}}^{\boldsymbol{7}}\right\rangle \cdot\left|\psi_{g}^{7}\right\rangle$ is defined in (3.16)

$$
\begin{aligned}
\left|\psi_{g}^{7}\right\rangle \equiv & -\frac{1}{2} \int_{\Lambda}^{e^{\delta \curlyvee} \Lambda} d k^{+} d p^{+} d q^{+} d r^{+} \int d^{2} \mathbf{k} d^{2} \mathbf{p} d^{2} \mathbf{q} d^{2} \mathbf{r} \\
& \times\left|g_{l}^{d}(k)\right\rangle \frac{\left\langle g_{l}^{d}(k)\left|H_{g}\right| g_{j}^{b}(r) g_{k}^{c}(q)\right\rangle\left\langle g_{j}^{b}(r) g_{k}^{c}(q)\left|H_{g}\right| g_{i}^{a}(p)\right\rangle\left\langle g_{i}^{a}(p)\left|H_{g}\right| 0\right\rangle}{E_{g}(k) E_{g g}(r, q) E_{g}(p)} .
\end{aligned}
$$




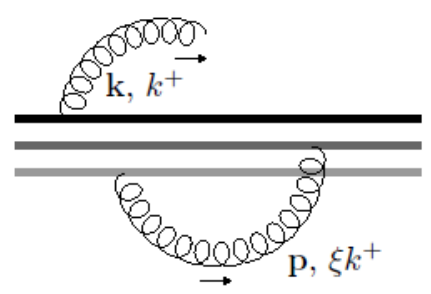

(a)

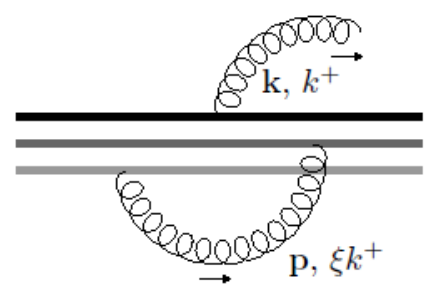

(b)

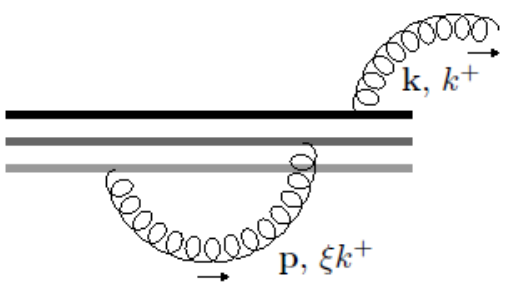

(c)

Figure 9. One gluon production diagrams with contribution to three $\rho$. (a,b) - $\left|\psi_{g}^{7}\right\rangle$, (c) - $\left|\psi_{g}^{8}\right\rangle$.

$\left|\psi_{g}^{7}\right\rangle$ accounts for two distinct processes depicted, after the dummy momenta are integrated over, in figure $9 \mathrm{a}$ and figure $9 \mathrm{~b}$. They correspond to two different light cone orderings in gluon emission/absorption process. Intuitively, we expect $\left|\psi_{g}^{7}\right\rangle$ to mostly contribute to $(\mathrm{LO})^{2}$ effects. Indeed the structure of singularities, see below, is consistent with our intuition. Particularly, we are to see a logarithmic divergence in the longitudinal integration, which can be identified as a virtual contribution at the LO, followed by emission of another soft gluon. The calculation is available in appendix G.6, from which we quote the result:

$$
\begin{aligned}
\left|\psi_{g}^{7}\right\rangle= & -\int_{\Lambda}^{e^{\delta \curlyvee} \Lambda} d k^{+} \int d^{2} \mathbf{k} d^{2} \mathbf{p} \int_{\frac{\Lambda}{k^{+}}}^{\frac{e^{\delta \curlyvee} \Lambda}{k^{+}}} d \xi \frac{g^{3} \mathbf{k}^{i} \rho^{b}(\mathbf{p})}{8 \pi^{9 / 2} \sqrt{k^{+}} \xi \mathbf{k}^{2}} \\
& \times\left(\frac{\rho^{b}(-\mathbf{p}) \rho^{a}(-\mathbf{k})}{\xi \mathbf{k}^{2}}+\frac{i f^{a b c} \rho^{c}(-\mathbf{k}-\mathbf{p})}{\xi \mathbf{k}^{2}+\mathbf{p}^{2}}\right)\left|g_{i}^{a}(k)\right\rangle,
\end{aligned}
$$

which can be written as a sum:

$$
\left|\psi_{g}^{7}\right\rangle=\left|\psi_{g \rho}^{7}\right\rangle+\left|\psi_{g \rho \rho}^{7}\right\rangle+\left|\psi_{g \rho \rho \rho}^{7}\right\rangle .
$$

- One $\rho$ part.

$$
\begin{aligned}
\left|\psi_{g \rho}^{7}\right\rangle \equiv & -\int_{\Lambda}^{e^{\delta \curlyvee} \Lambda} d k^{+} \int d^{2} \mathbf{k} \frac{g^{3} N_{c} \rho^{a}(-\mathbf{k}) \mathbf{k}^{i}}{32 \pi^{7 / 2} \sqrt{k^{+}} \mathbf{k}^{2}} \\
& \times\left(2 \delta \mathrm{Y}\left[-\frac{2}{\epsilon}+\ln \left(\frac{\mathbf{k}^{2}}{\mu_{M S}^{2}}\right)\right]+\ln ^{2}\left(\frac{\Lambda e^{\delta \curlyvee}}{k^{+}}\right)-\ln ^{2}\left(\frac{\Lambda}{k^{+}}\right)\right)\left|g_{i}^{a}(k)\right\rangle .
\end{aligned}
$$

- Two $\boldsymbol{\rho}$ part. This part can be directly deduced from (3.73):

$$
\left|\psi_{g \rho \rho}^{7}\right\rangle \equiv-\int_{\Lambda}^{e^{\delta \curlyvee} \Lambda} d k^{+} \int d^{2} \mathbf{k} d^{2} \mathbf{p} \int_{\frac{\Lambda}{k^{+}}}^{\frac{e^{\delta \curlyvee}}{k^{+}}} d \xi \frac{i g^{3} f^{a b c} \mathbf{k}^{i}\left\{\rho^{b}(-\mathbf{p}), \rho^{c}(-\mathbf{k}+\mathbf{p})\right\}}{16 \pi^{9 / 2} \sqrt{k^{+}} \xi \mathbf{k}^{2}\left(\xi \mathbf{k}^{2}+\mathbf{p}^{2}\right)}\left|g_{i}^{a}(k)\right\rangle .
$$

After integration over $\xi \equiv \frac{p^{+}}{k^{+}}$according to (C.17):

$$
\begin{aligned}
\left|\psi_{g \rho \rho}^{7}\right\rangle= & \int_{\Lambda}^{e^{\delta \curlyvee} \Lambda} d k^{+} \int d^{2} \mathbf{k} d^{2} \mathbf{p} \frac{i g^{3} f^{a b c} \mathbf{k}^{i}\left\{\rho^{b}(-\mathbf{p}), \rho^{c}(-\mathbf{k}+\mathbf{p})\right\}}{16 \pi^{9 / 2} \sqrt{k^{+}} \mathbf{k}^{2} \mathbf{p}^{2}} \\
& \times\left(\ln \left(\frac{\Lambda}{k^{+}}\right)-\ln \left(\frac{\mathbf{p}^{2}}{\mathbf{k}^{2}}\right)\right)\left|g_{i}^{a}(k)\right\rangle .
\end{aligned}
$$


Another useful representation is obtained by changing $p \rightarrow k-p$ in the second term,

$$
\begin{aligned}
\left|\psi_{g \rho \rho}^{7}\right\rangle= & \int_{\Lambda}^{e^{\delta \curlyvee} \Lambda} d k^{+} \int d^{2} \mathbf{k} d^{2} \mathbf{p} \frac{i g^{3} f^{a b c} \mathbf{k}^{i}\left\{\rho^{b}(-\mathbf{p}), \rho^{c}(-\mathbf{k}+\mathbf{p})\right\}}{16 \pi^{9 / 2} \sqrt{k^{+}} \mathbf{k}^{2}} \\
& \times\left(\frac{1}{\mathbf{p}^{2}} \ln \left(\frac{\Lambda}{k^{+}}\right)+\frac{1}{(\mathbf{k}-\mathbf{p})^{2}} \ln \left(\frac{(\mathbf{k}-\mathbf{p})^{2}}{\mathbf{k}^{2}}\right)\right)\left|g_{i}^{a}(k)\right\rangle .
\end{aligned}
$$

- Three $\boldsymbol{\rho}$ part. This part can be directly deduced from (3.73):

$$
\left|\psi_{g \rho \rho \rho}^{7}\right\rangle \equiv-\int_{\Lambda}^{e^{\delta \curlyvee} \Lambda} d k^{+} \int d^{2} \mathbf{k} d^{2} \mathbf{p} \int_{\frac{\Lambda}{k^{+}}}^{\frac{e^{\delta \Upsilon_{\Lambda}}}{k^{+}}} d \xi \frac{g^{3} \mathbf{k}^{i} \rho^{b}(\mathbf{p}) \rho^{b}(-\mathbf{p}) \rho^{a}(-\mathbf{k})}{8 \pi^{9 / 2} \sqrt{k^{+}} \xi^{2} \mathbf{k}^{4}}\left|g_{i}^{a}(k)\right\rangle .
$$

After integration over $\xi$ :

$$
\left|\psi_{g \rho \rho \rho}^{7}\right\rangle \equiv \int_{\Lambda}^{e^{\delta \curlyvee} \Lambda} d k^{+} \int d^{2} \mathbf{k} d^{2} \mathbf{p} \frac{g^{3} \mathbf{k}^{i} \rho^{b}(\mathbf{p}) \rho^{b}(-\mathbf{p}) \rho^{a}(-\mathbf{k}) \sqrt{k^{+}}}{8 \pi^{9 / 2} \mathbf{k}^{4} \Lambda}\left(\frac{1}{e^{\delta \curlyvee}}-1\right)\left|g_{i}^{a}(k)\right\rangle .
$$

From the general structure of the NLO JIMWLK Hamiltonian, we know that $\left|\psi_{g \rho \rho \rho}^{7}\right\rangle$ is not expected to contribute to it. At the level of Hamiltonian, $\left|\psi_{g \rho \rho \rho}^{7}\right\rangle$ gives rise to $\rho^{4}$ terms, which are not present in the NLO JIMWLK Hamiltonian. Consistency demands that all such terms are expected to be absorbed into (LO) ${ }^{2}$ Hamiltonian. After a trivial cancelation against $\left|\psi_{g}^{8}\right\rangle$, which is to be computed next, a combined contribution is indeed found of the right form.

Computation of $\left|\boldsymbol{\psi}_{\boldsymbol{g}}^{\mathbf{8}}\right\rangle \cdot\left|\psi_{g}^{8}\right\rangle$ is defined in (3.17) (figure 9c):

$\left|\psi_{g}^{8}\right\rangle \equiv \int_{\Lambda}^{e^{\delta \curlyvee} \Lambda} d k^{+} d p^{+} \int d^{2} \mathbf{k} d^{2} \mathbf{p}\left|g_{i}^{a}(k)\right\rangle \frac{\left|\left\langle g_{j}^{b}(p)\left|H_{g}\right| 0\right\rangle\right|^{2}\left\langle g_{i}^{a}(k)\left|H_{g}\right| 0\right\rangle\left(2 E_{g}(p)+E_{g}(k)\right)}{2 E_{g}^{2}(k) E_{g}^{2}(p)}$.

After inserting the relevant matrix element (2.49) and changing variables according to (3.34), we arrive at:

$$
\left|\psi_{g}^{8}\right\rangle=\int_{\Lambda}^{e^{\delta \curlyvee} \Lambda} d k^{+} \int d^{2} \mathbf{k} d^{2} \mathbf{p} \int_{\frac{\Lambda}{k^{+}}}^{\frac{e^{\delta \curlyvee}}{k^{+}}} d \xi \frac{g^{3} \mathbf{k}^{i} \rho^{b}(\mathbf{p}) \rho^{b}(-\mathbf{p}) \rho^{a}(-\mathbf{k})}{16 \pi^{9 / 2} \sqrt{k^{+}} \xi^{2} \mathbf{k}^{2}}\left(\frac{\xi}{\mathbf{p}^{2}}+\frac{2}{\mathbf{k}^{2}}\right)\left|g_{i}^{a}(k)\right\rangle .
$$

After integration over $\xi$ :

$$
\begin{aligned}
& \left|\psi_{g \rho \rho \rho}^{8}\right\rangle \equiv\left|\psi_{g}^{8}\right\rangle \\
& \quad=\int_{\Lambda}^{e^{\delta \Upsilon} \Lambda} d k^{+} \int d^{2} \mathbf{k} d^{2} \mathbf{p} \frac{g^{3} \mathbf{k}^{i} \rho^{b}(\mathbf{p}) \rho^{b}(-\mathbf{p}) \rho^{a}(-\mathbf{k})}{16 \pi^{9 / 2} \sqrt{k^{+}} \mathbf{k}^{2}}\left(\frac{\delta \mathbf{Y}}{\mathbf{p}^{2}}-\left(\frac{1}{e^{\delta \mathbf{Y}}}-1\right) \frac{2 k^{+}}{\Lambda \mathbf{k}^{2}}\right)\left|g_{i}^{a}(k)\right\rangle .
\end{aligned}
$$




\subsection{The final result}

In this section we will assemble together all the different contributions that were computed in section 3.4. It turns out to be useful to replace the representation of the LCFW as in (??) by a new representation schematically outlined in (1.2), in which the classification of the contributions will be made according to the soft particle content $(q \bar{q}, g g$ or $g)$ and the number of valence current operators $(\rho, \rho \rho$ or $\rho \rho \rho)$. This information will be indicated by a subscript. For example, the state $\left|\psi_{\text {gg } \rho \rho}\right\rangle$ contains two soft gluons and two $\rho$ operators. The NLO wave function is decomposed as follows:

$$
\left|\psi^{\mathrm{NLO}}\right\rangle=\mathcal{N}^{\mathrm{NLO}}|0\rangle+\left|\psi_{g \rho}^{L O}\right\rangle+\left|\psi_{q \bar{q} \rho}\right\rangle+\left|\psi_{g g}\right\rangle+\left|\psi_{g g} \rho \rho\right|+\left|\psi_{g \rho}\right\rangle+\left|\psi_{g \rho \rho}\right\rangle+\left|\psi_{g \rho \rho \rho}\right\rangle .
$$

$\left|\psi_{g \rho}^{L O}\right\rangle$ was defined in (3.4). Additional states are:

$$
\begin{aligned}
\left|\psi_{q \bar{q} \rho}\right\rangle & \equiv\left|\psi_{q \bar{q} \rho}^{1}\right\rangle+\left|\psi_{q \bar{q} \rho}^{2}\right\rangle, \\
\left|\psi_{g g}\right\rangle & \equiv\left|\psi_{g g \rho}^{1}\right\rangle+\left|\psi_{g g \rho}^{2}\right\rangle+\left|\psi_{g g \rho}^{3}\right\rangle, \\
\left|\psi_{g \rho}\right\rangle & \equiv\left|\psi_{g \rho}^{1}\right\rangle+\left|\psi_{g \rho}^{2}\right\rangle+\left|\psi_{g \rho}^{4+5}\right\rangle+\left|\psi_{g \rho}^{6}\right\rangle+\left|\psi_{g \rho}^{7}\right\rangle, \\
\left|\psi_{g \rho \rho}\right\rangle & \equiv\left|\psi_{g \rho \rho}^{3 u}\right\rangle+\left|\psi_{g \rho \rho}^{3 d}\right\rangle+\left|\psi_{g \rho \rho}^{4+5}\right\rangle+\left|\psi_{g \rho \rho}^{6}\right\rangle+\left|\psi_{g \rho \rho}^{7}\right\rangle, \\
\left|\psi_{g \rho \rho \rho}\right\rangle & \equiv\left|\psi_{g \rho \rho \rho}^{7}\right\rangle+\left|\psi_{g \rho \rho \rho}^{8}\right\rangle .
\end{aligned}
$$

- The quark and anti-quark state. Inserting (3.33) and (3.36) to (3.85), and changing the measure according to (3.28), yeilds:

$$
\begin{aligned}
\left|\psi_{q \bar{q} \rho}\right\rangle= & \sum_{\lambda_{1}, \lambda_{2}, f} \int_{\Lambda}^{e^{\delta \curlyvee} \Lambda} d k^{+} \int_{0}^{1} d \xi \int \frac{d^{2} \mathbf{k}}{(2 \pi)^{2}} \frac{d^{2} \mathbf{p}}{(2 \pi)^{2}} \frac{2 \pi g^{2} t_{\alpha \beta}^{a} \rho^{a}(-\mathbf{k}) \xi(1-\xi)}{(1-\xi) \mathbf{p}^{2}+\xi(\mathbf{k}-\mathbf{p})^{2}} \\
& \times \chi_{\lambda_{1}}^{\dagger}\left(\frac{\mathbf{k}^{i}}{\mathbf{k}^{2}}\left[2 \mathbf{k}^{i}-\frac{\sigma \cdot \mathbf{p}}{\xi} \sigma^{i}-\sigma^{i} \frac{\sigma \cdot(\mathbf{k}-\mathbf{p})}{1-\xi}\right]-2\right) \chi_{\lambda_{2}}\left|\bar{q}_{\lambda_{2}}^{\beta, f}(k-p) q_{\lambda_{1}}^{\alpha, f}(p)\right\rangle,
\end{aligned}
$$

where $p^{+}=\xi k^{+}$. Another representation is obtained after introducing the variable $\widetilde{\mathbf{p}}$ according to (3.45):

$$
\begin{aligned}
\left|\psi_{q \bar{q} \rho}\right\rangle= & -\sum_{\lambda_{1}, \lambda_{2}, f} \int_{\Lambda}^{e^{\delta \curlyvee} \Lambda} d k^{+} \int_{0}^{1} d \xi \int \frac{d^{2} \mathbf{k}}{(2 \pi)^{2}} \frac{d^{2} \mathbf{p}}{(2 \pi)^{2}} \frac{2 \pi g^{2} t_{\alpha \beta}^{a} \rho^{a}(-\mathbf{k})}{\xi(1-\xi) \mathbf{k}^{2}+\widetilde{\mathbf{p}}^{2}} \\
& \times \chi_{\lambda_{1}}^{\dagger}\left(\frac{\mathbf{k}^{i} \widetilde{\mathbf{p}}^{j}}{\mathbf{k}^{2}}\left[(1-2 \xi) \delta^{i j}-i \varepsilon^{i j k} \sigma^{k}\right]+2 \xi(1-\xi)\right) \chi_{\lambda_{2}} \\
& \times\left|\bar{q}_{\lambda_{2}}^{\beta, f}\left((1-\xi) k^{+},(1-\xi) \mathbf{k}-\widetilde{\mathbf{p}}\right) q_{\lambda_{1}}^{\alpha, f}\left(\xi k^{+}, \xi \mathbf{k}+\widetilde{\mathbf{p}}\right)\right\rangle .
\end{aligned}
$$

Transforming to coordinate space using (C.23) and (C.24),

$$
\begin{gathered}
\left|\psi_{q \bar{q} \rho}\right\rangle=-\sum_{\lambda_{1}, \lambda_{2}, f} \int_{\mathbf{x}, \mathbf{z}, \mathbf{z}^{\prime}} \int_{\Lambda}^{e^{\delta \curlyvee} \Lambda} d k^{+} \int_{0}^{1} d \xi \frac{g^{2} t_{\alpha \beta}^{a} \rho^{a}(\mathbf{x})}{8 \pi^{3}\left((1-\xi)\left(X^{\prime}\right)^{2}+\xi X^{2}\right)} \chi_{\lambda_{1}}^{\dagger}\left(\frac{\left(X^{\prime}-\xi Z\right)^{i} Z^{j}}{Z^{2}}\right. \\
\left.\times\left[(1-2 \xi) \delta^{i j}-i \varepsilon^{i j k} \sigma^{k}\right]+2 \xi(1-\xi)\right) \chi_{\lambda_{2}}\left|\bar{q}_{\lambda_{2}}^{\beta, f}\left((1-\xi) k^{+}, \mathbf{z}^{\prime}\right) q_{\lambda_{1}}^{\alpha, f}\left(\xi k^{+}, \mathbf{z}\right)\right\rangle \cdot
\end{gathered}
$$


- The two gluon state with one $\boldsymbol{\rho}$. By inserting (3.41), (3.46), and (3.48) to (3.86) we arrive at:

$$
\begin{aligned}
\left|\psi_{g g \rho}\right\rangle= & \int_{\Lambda}^{e^{\delta \curlyvee} \Lambda} d k^{+} \int d^{2} \mathbf{k} d^{2} \widetilde{\mathbf{p}} \int_{\frac{\Lambda}{k^{+}}}^{1-\frac{\Lambda}{k^{+}}} d \xi \frac{i g^{2} f^{a b c} \rho^{a}(-\mathbf{k}) \sqrt{\xi(1-\xi)}}{8 \pi^{3}\left(\mathbf{k}^{2} \xi(1-\xi)+\widetilde{\mathbf{p}}^{2}\right) \mathbf{k}^{2}} \\
& \times\left(\frac{\mathbf{k}^{2}\left(\xi \mathbf{k}^{i}+\widetilde{\mathbf{p}}^{i}\right)\left((1-\xi) \mathbf{k}^{j}-\widetilde{\mathbf{p}}^{j}\right)\left(\xi((1-\xi) \mathbf{k}-\widetilde{\mathbf{p}})^{2}-(1-\xi)(\xi \mathbf{k}+\widetilde{\mathbf{p}})^{2}\right)}{2 \xi(1-\xi)(\xi \mathbf{k}+\widetilde{\mathbf{p}})^{2}((1-\xi) \mathbf{k}-\widetilde{\mathbf{p}})^{2}}\right. \\
& \left.+\frac{\delta^{j l}}{2}\left((1-2 \xi) \mathbf{k}^{2}-2 \mathbf{k} \cdot \widetilde{\mathbf{p}}\right)+\frac{1}{\xi} \mathbf{k}^{j} \widetilde{\mathbf{p}}^{l}+\frac{1}{1-\xi} \mathbf{k}^{l} \widetilde{\mathbf{p}}^{j}\right) \\
& \times\left|g_{j}^{b}\left((1-\xi) k^{+},(1-\xi) \mathbf{k}-\widetilde{\mathbf{p}}\right) g_{l}^{c}\left(\xi k^{+}, \xi \mathbf{k}+\widetilde{\mathbf{p}}\right)\right\rangle
\end{aligned}
$$

Transforming to coordinate space using (C.23) and (C.24):

$$
\begin{aligned}
\left|\psi_{g g \rho}\right\rangle= & -\int_{\mathbf{x}, \mathbf{z}, \mathbf{z}^{\prime}} \int_{\Lambda}^{e^{\delta \curlyvee} \Lambda} d k^{+} \int_{\frac{\Lambda}{k^{+}}}^{1-\frac{\Lambda}{k^{+}}} d \xi \frac{i g^{2} f^{a b c} \rho^{a}(\mathbf{x}) \sqrt{\xi(1-\xi)}}{8 \pi^{3}\left((1-\xi)\left(X^{\prime}\right)^{2}+\xi X^{2}\right)} \\
& \times\left(\frac{\delta^{j l}}{2 Z^{2}}\left(X^{2}-\left(X^{\prime}\right)^{2}\right)+\frac{\left(X^{\prime}\right)^{j} Z^{l}}{\xi Z^{2}}+\frac{X^{l} Z^{j}}{(1-\xi) Z^{2}}+\frac{X^{l}\left(X^{\prime}\right)^{j}}{2 \xi X^{2}}-\frac{X^{l}\left(X^{\prime}\right)^{j}}{2(1-\xi)\left(X^{\prime}\right)^{2}}\right) \\
& \times\left|g_{l}^{b}\left(\xi k^{+}, \mathbf{z}\right) g_{j}^{c}\left((1-\xi) k^{+}, \mathbf{z}^{\prime}\right)\right\rangle .
\end{aligned}
$$

- The two gluon state with two $\boldsymbol{\rho}$. This result was shown in (3.42):

$$
\begin{aligned}
\left|\psi_{g g \rho \rho}\right\rangle= & \int_{\Lambda}^{e^{\delta \curlyvee} \Lambda} d k^{+} \int d^{2} \mathbf{k} d^{2} \mathbf{p} \int_{\frac{\Lambda}{k^{+}}}^{1-\frac{\Lambda}{k^{+}}} d \xi \\
& \times \frac{g^{2} \mathbf{p}^{i}\left(\mathbf{k}^{j}-\mathbf{p}^{j}\right)\left\{\rho^{a}(-\mathbf{p}), \rho^{b}(-\mathbf{k}+\mathbf{p})\right\}}{16 \pi^{3} \mathbf{p}^{2}(\mathbf{k}-\mathbf{p})^{2} \sqrt{\xi(1-\xi)}}\left|g_{j}^{b}(k-p) g_{i}^{a}(p)\right\rangle .
\end{aligned}
$$

After transformation to coordinate space using (C.21):

$$
\begin{aligned}
\left|\psi_{g g \rho \rho}\right\rangle= & -\int_{\mathbf{x}, \mathbf{y}, \mathbf{z}, \mathbf{z}^{\prime}} \int_{\Lambda}^{e^{\delta \curlyvee} \Lambda} d k^{+} \int_{\frac{\Lambda}{k^{+}}}^{1-\frac{\Lambda}{k^{+}}} d \xi \\
& \times \frac{g^{2}\left\{\rho^{a}(\mathbf{y}), \rho^{b}(\mathbf{x})\right\} Y^{i}\left(X^{\prime}\right)^{j}}{16 \pi^{3} \sqrt{\xi(1-\xi)} Y^{2}\left(X^{\prime}\right)^{2}}\left|g_{j}^{b}\left((1-\xi) k^{+}, \mathbf{z}^{\prime}\right) g_{i}^{a}\left(\xi k^{+}, \mathbf{z}\right)\right\rangle .
\end{aligned}
$$

- The one gluon state with one $\boldsymbol{\rho}$. By inserting (3.50), (3.52), (3.64), (3.70), and (3.75) to $(3.87)$ we arrive at:

$$
\begin{aligned}
\left|\psi_{g}\right\rangle= & \int_{\Lambda}^{e^{\delta \curlyvee} \Lambda} d k^{+} \int d^{2} \mathbf{k} \frac{g^{3} \rho^{a}(-\mathbf{k}) \mathbf{k}^{i}}{32 \pi^{7 / 2} \mathbf{k}^{2} \sqrt{k^{+}}}\left[\left(\left[b+N_{c} \ln \left(\frac{\Lambda}{k^{+}}\right)-2 N_{c} \delta \mathbf{Y}\right]\right.\right. \\
& \times\left[-\frac{2}{\epsilon}+\ln \left(\frac{\mathbf{k}^{2}}{\mu^{2}}\right)\right]-N_{c} \ln ^{2}\left(\frac{\Lambda e^{\delta \mathbf{Y}}}{k^{+}}\right)+N_{c} \ln ^{2}\left(\frac{\Lambda}{k^{+}}\right)-\left(\frac{67}{9}-\frac{\pi^{2}}{3}\right) N_{c} \\
& \left.+\frac{10}{9} N_{f}\right)\left|g_{i}^{a}(k)\right\rangle+\int_{\frac{\Lambda}{k^{+}}}^{1-\frac{\Lambda}{k^{+}}} d \xi \frac{N_{c}}{\sqrt{\xi}(1-\xi)}((\xi-2) \ln (\xi)+(\xi+1) \ln (1-\xi) \\
& \left.\left.-(\xi-2)\left[-\frac{2}{\epsilon}+\ln \left(\frac{\mathbf{k}^{2}}{\mu_{\frac{2}{M S}}^{2}}\right)\right]\right)\left|g_{i}^{a}\left(\xi k^{+}, \mathbf{k}\right)\right\rangle\right] .
\end{aligned}
$$


where the parameter $b$ was defined in (2.65) and $\mu_{\overline{M S}}^{2} \equiv 4 \pi e^{-\gamma} \mu^{2}$. The transformation to coordinate space is possible using (C.21) and (C.22):

$$
\begin{aligned}
\left|\psi_{g \rho}\right\rangle= & -\int_{\mathbf{x}, \mathbf{z}} \int_{\Lambda}^{e^{\delta \Upsilon} \Lambda} d k^{+} \frac{i g^{3} \rho^{a}(\mathbf{x}) X^{i}}{32 \pi^{7 / 2} X^{2} \sqrt{k^{+}}}\left[\left([ b + N _ { c } \operatorname { l n } ( \frac { \Lambda } { k ^ { + } } ) - 2 N _ { c } \delta \mathbf { Y } ] \left[-\frac{2}{\epsilon}-2 \gamma\right.\right.\right. \\
& \left.-\ln \left(\frac{X^{2} \mu_{\overline{M S}}^{2}}{4}\right)\right]-N_{c} \ln ^{2}\left(\frac{\Lambda e^{\delta \mathbf{Y}}}{k^{+}}\right)+N_{c} \ln ^{2}\left(\frac{\Lambda}{k^{+}}\right)-\left(\frac{67}{9}-\frac{\pi^{2}}{3}\right) N_{c} \\
& \left.+\frac{10}{9} N_{f}\right)\left|g_{i}^{a}\left(k^{+}, \mathbf{z}\right)\right\rangle+\int_{\frac{\Lambda}{k^{+}}}^{1-\frac{\Lambda}{k^{+}}} d \xi \frac{N_{c}}{\sqrt{\xi}(1-\xi)}((\xi-2) \ln (\xi)+(\xi+1) \ln (1-\xi) \\
& \left.\left.-(\xi-2)\left[-\frac{2}{\epsilon}-2 \gamma-\ln \left(\frac{X^{2} \mu_{\overline{M S}}^{2}}{4}\right)\right]\right)\left|g_{i}^{a}\left(\xi k^{+}, \mathbf{z}\right)\right\rangle\right] .
\end{aligned}
$$

In section 4, we will use this form of $\left|\psi_{g \rho}\right\rangle$. However, after defining a new variable $\bar{k}^{+}=\xi k^{+}$, one of the longitudinal integrals can be evaluated. For completeness of the presentation, we also quote this result:

$$
\begin{aligned}
& \left|\psi_{g \rho}\right\rangle=-\int_{\mathbf{x}, \mathbf{z}} \int_{\Lambda}^{e^{\delta \Upsilon} \Lambda} d k \frac{i g^{3} \rho^{a}(\mathbf{x}) X^{i}}{32 \pi^{7 / 2} X^{2} \sqrt{k^{+}}}\left\{\left[b+N_{c} \ln \left(\frac{\Lambda}{k^{+}}\right)+N_{c} \ln \left(\frac{\Lambda e^{\delta \mathbf{Y}}}{k^{+}}-1\right)-N_{c} \delta \mathbf{Y}\right]\right. \\
& \times\left[-\frac{2}{\epsilon}-2 \gamma-\ln \left(\frac{X^{2} \mu_{\overline{M S}}^{2}}{4}\right)\right]+\frac{10}{9} N_{f}+N_{c}\left[\frac{\pi^{2}}{3}-\frac{67}{9}+\ln ^{2}\left(1-\frac{k^{+}}{\Lambda e^{\delta \mathbf{Y}}}\right)+\ln \left(1-\frac{k^{+}}{\Lambda e^{\delta \mathbf{Y}}}\right)\right. \\
& \left.\left.\times \ln \left(\frac{\Lambda e^{\delta \mathbf{Y}}}{k^{+}}\right)\right]\right\}\left|g_{i}^{a}\left(k^{+}, \mathbf{z}\right)\right\rangle
\end{aligned}
$$

$\left|\psi_{g} \rho\right\rangle$ appears to be one of the most non-trivial parts of our calculation, which to our knowledge has not been computed before. It has a mixture of various terms, including singular ones. It is hard to give interpretation to every term at the level of the LCWF. We only comment here that $\left.\left|\psi_{g}\right\rangle\right\rangle$ contributes both to NLO JIMWLK Hamiltonian (the kernel $\left.K_{\mathrm{JSJ}}\right)$ and second iteration of the LO one. $\left|\psi_{g}\right\rangle$ is also a source of the $\beta$-function of the running coupling.

- The one gluon state with two $\boldsymbol{\rho}$. By inserting (3.56), (3.59), (3.66), (3.71), and (3.76) to $(3.88)$ we arrive at:

$$
\begin{aligned}
\left|\psi_{g \rho \rho}\right\rangle \equiv & \int_{\Lambda}^{e^{\delta \curlyvee} \Lambda} d k^{+} \int d^{2} \mathbf{k} d^{2} \mathbf{p} \frac{i g^{3} f^{a b c}\left\{\rho^{b}(-\mathbf{p}), \rho^{c}(-\mathbf{k}+\mathbf{p})\right\}}{32 \pi^{9 / 2} \mathbf{p}^{2} \mathbf{k}^{2} \sqrt{k^{+}}}\left\{\left(\left[\left(2-\frac{\mathbf{k} \cdot \mathbf{p}}{(\mathbf{k}-\mathbf{p})^{2}}\right)\right.\right.\right. \\
& \left.\left.\times \ln \left(\frac{\Lambda}{k^{+}}\right)+\frac{2 \mathbf{p}^{2}}{(\mathbf{k}-\mathbf{p})^{2}} \ln \left(\frac{(\mathbf{k}-\mathbf{p})^{2}}{\mathbf{k}^{2}}\right)\right] \mathbf{k}^{i}+\ln \left(\frac{\Lambda}{k^{+}}\right) \frac{\mathbf{k}^{2}}{(\mathbf{k}-\mathbf{p})^{2}} \mathbf{p}^{i}\right)\left|g_{i}^{a}(k)\right\rangle \\
& -\int_{\frac{\Lambda}{k^{+}}}^{1-\frac{\Lambda}{k^{+}}} d \xi \frac{\xi^{3 / 2}}{\left(\xi(\mathbf{k}-\mathbf{p})^{2}+(1-\xi) \mathbf{k}^{2}\right)(1-\xi)}\left(\left[\frac{2}{\xi}\left(\mathbf{p}^{2}-\mathbf{k} \cdot \mathbf{p}\right)-\mathbf{p}^{2}+2 \mathbf{k} \cdot \mathbf{p}\right] \mathbf{k}^{i}\right. \\
& \left.\left.+\frac{1}{\xi} \mathbf{k}^{2} \mathbf{p}^{i}\right)\left|g_{i}^{a}\left(\xi k^{+}, \mathbf{k}\right)\right\rangle\right\} .
\end{aligned}
$$


Again, despite the possibility to evaluate one of the integrals, in section 4, we will use this form of $\left|\psi_{g} \rho \rho\right\rangle$. For completeness of presentation, we also quote the final result:

$$
\begin{aligned}
\left|\psi_{g \rho \rho}\right\rangle \equiv & \int_{\Lambda}^{e^{\delta \curlyvee} \Lambda} d k^{+} \int d^{2} \mathbf{k} d^{2} \mathbf{p} \frac{i g^{3} f^{a b c}\left\{\rho^{b}(-\mathbf{p}), \rho^{c}(-\mathbf{k}+\mathbf{p})\right\}}{32 \pi^{9 / 2} \mathbf{p}^{2} \mathbf{k}^{2} \sqrt{k^{+}}}\left\{\left[\left(2-\frac{\mathbf{k} \cdot \mathbf{p}}{(\mathbf{k}-\mathbf{p})^{2}}\right)\right.\right. \\
& \left.\times \ln \left(\frac{\Lambda}{k^{+}}\right)+\frac{2 \mathbf{p}^{2}}{(\mathbf{k}-\mathbf{p})^{2}} \ln \left(\frac{(\mathbf{k}-\mathbf{p})^{2}}{\mathbf{k}^{2}}\right)\right] \mathbf{k}^{i}+\ln \left(\frac{\Lambda}{k^{+}}\right) \frac{\mathbf{k}^{2}}{(\mathbf{k}-\mathbf{p})^{2}} \mathbf{p}^{i} \\
& +\frac{\left(2 \mathbf{p}^{2}-2 \mathbf{k} \cdot \mathbf{p}+\mathbf{k}^{2}\right) \mathbf{k}^{i}+\mathbf{k}^{2} \mathbf{p}^{i}}{(\mathbf{k}-\mathbf{p})^{2}} \ln \left(1+\left[\frac{e^{\delta \curlyvee} \Lambda}{k^{+}}-1\right] \frac{\mathbf{k}^{2}}{(\mathbf{k}-\mathbf{p})^{2}}\right)-\frac{\mathbf{p}^{2} \mathbf{k}^{i}+\mathbf{k}^{2} \mathbf{p}^{i}}{(\mathbf{k}-\mathbf{p})^{2}} \\
& \left.\times \ln \left(e^{\delta \curlyvee}-\frac{k^{+}}{\Lambda}\right)-\mathbf{k}^{i} \ln \left(\frac{e^{\delta \curlyvee} \Lambda}{k^{+}}\right)\right\}\left|g_{i}^{a}(k)\right\rangle
\end{aligned}
$$

We did not manage to compute a coordinate representation for this component. Together with $\left|\psi_{g} \rho\right\rangle,\left|\psi_{g \rho \rho}\right\rangle$ constitutes one of the main new non-trivial result. In the next section, we will demonstrate how $\left|\psi_{g \rho \rho}\right\rangle$ contributes both to NLO JIMWLK Hamiltonian and the LO iteration. Particularly $\left|\psi_{g} \rho \rho\right\rangle$ will give rise to the NLO kernel $K_{\text {JJSJ }}$. The kernel $K_{\text {JJSJ }}$ is related to the kernel $K_{\text {JJSSJ }}$ through the equation (D.4). In its turn, the kernel $K_{\text {JJSSJ }}$ will be obtained from $\left|\psi_{g g} \rho \rho\right\rangle$ (3.42). We notice that while there is a relation between the kernels, it does not seem to be possible to establish any interesting relation at the level of the LCWF components.

- The one gluon state with three $\boldsymbol{\rho}$. Substituting (3.80) and (3.83) in (3.89), we arrive at:

$$
\left|\psi_{g \rho \rho \rho}\right\rangle=\delta \mathrm{Y} \int_{\Lambda}^{e^{\delta \curlyvee} \Lambda} d k^{+} \int d^{2} \mathbf{k} d^{2} \mathbf{p} \frac{g^{3} \rho^{b}(\mathbf{p}) \rho^{b}(-\mathbf{p}) \rho^{a}(-\mathbf{k}) \mathbf{k}^{i}}{16 \pi^{9 / 2} \mathbf{k}^{2} \mathbf{p}^{2} \sqrt{k^{+}}}\left|g_{i}^{a}(k)\right\rangle .
$$

Inserting the identity $\int d^{2} \mathbf{q} \frac{\mathbf{p} \cdot \mathbf{q}}{\mathbf{q}^{2}} \delta^{(2)}(\mathbf{q}-\mathbf{p})=1$ in (3.102), the latter can be straightforwardly written in coordinate space:

$$
\left|\psi_{g \rho \rho \rho}\right\rangle=-\delta \mathbf{Y} \int_{\mathbf{w}, \mathbf{x}, \mathbf{y}, \mathbf{z}, \mathbf{z}^{\prime}} \int_{\Lambda}^{e^{\delta \curlyvee} \Lambda} d k^{+} \frac{i g^{3} \rho^{b}(\mathbf{x}) \rho^{b}(\mathbf{y}) \rho^{a}(\mathbf{w}) X \cdot Y\left(W^{\prime}\right)^{i}}{16 \pi^{9 / 2} X^{2} Y^{2}\left(W^{\prime}\right)^{2} \sqrt{k^{+}}}\left|g_{i}^{a}\left(k^{+}, \mathbf{z}^{\prime}\right)\right\rangle .
$$

The $\left|\psi_{g \rho \rho \rho}\right\rangle$ component of the LCWF is pretty transparent. It is already proportional to $\delta Y$ because of the virtual soft gluon, which we have integrated over. One can recognise in the integrand of (3.103) the kernel of the LO JIMWLK Hamiltonian. The extra soft gluon $g(k)$ in $\left|\psi_{g \rho \rho \rho}\right\rangle$ corresponds to another iteration of the kernel as will be demonstrated in the next section.

- Normalisation. The norm of the wave function can be represented as:

$$
\mathcal{N}^{\mathrm{NLO}}=\left\|\mathcal{N}^{\mathrm{NLO}}\right\| e^{i \phi^{\mathrm{NLO}}} .
$$


From normalisation condition on the expansion (3.84),

$$
\begin{aligned}
& \left\|\mathcal{N}^{\mathrm{NLO}}\right\|=1-\frac{1}{2}\left(\left\langle\psi_{g \rho}^{L O} \mid \psi_{g \rho}^{L O}\right\rangle+\left\langle\psi_{q \bar{q} \rho} \mid \psi_{q \bar{q} \rho}\right\rangle+\left\langle\psi_{g \rho}^{L O} \mid \psi_{g \rho}\right\rangle+\left\langle\psi_{g \rho} \mid \psi_{g \rho}^{L O}\right\rangle\right. \\
& \quad+\left\langle\psi_{g \rho}^{L O} \mid \psi_{g \rho \rho}\right\rangle+\left\langle\psi_{g \rho \rho} \mid \psi_{g \rho}^{L O}\right\rangle+\left\langle\psi_{g g \rho} \mid \psi_{g g \rho}\right\rangle+\left\langle\psi_{g g \rho} \mid \psi_{g g \rho \rho}\right\rangle+\left\langle\psi_{g g \rho \rho} \mid \psi_{g g \rho}\right\rangle \\
& \left.\quad+\left\langle\psi_{g g \rho \rho} \mid \psi_{g g \rho \rho}\right\rangle+\left\langle\psi_{g \rho}^{L O} \mid \psi_{g \rho \rho \rho}\right\rangle+\left\langle\psi_{g \rho \rho \rho} \mid \psi_{g \rho}^{L O}\right\rangle\right)-\frac{1}{8}\left\langle\psi_{g \rho}^{L O} \mid \psi_{g \rho}^{L O}\right\rangle\left\langle\psi_{g \rho}^{L O} \mid \psi_{g \rho}^{L O}\right\rangle .
\end{aligned}
$$

The computation relevant for $\left\|\mathcal{N}^{\mathrm{NLO}}\right\|$ is postponed until section 4.7. Here we quote the final result:

$$
\begin{aligned}
\| \mathcal{N}^{\mathrm{NLO} \|=} & 1-\frac{\alpha_{s}}{2 \pi^{2}} \delta \mathrm{Y} \int_{\mathbf{x}, \mathbf{y}, \mathbf{z}, \mathbf{z}^{\prime}} \frac{X \cdot Y}{X^{2} Y^{2}} \rho^{a}(\mathbf{x}) \rho^{a}(\mathbf{y})-\delta \mathbf{Y} \int_{\mathbf{x}, \mathbf{y}, \mathbf{z}} K_{\mathrm{JSJ}}(\mathbf{x}, \mathbf{y}, \mathbf{z}) \rho^{a}(\mathbf{x}) \rho^{a}(\mathbf{y}) \\
& +\frac{\alpha_{s}^{2}}{8 \pi^{4}}(\delta \mathbf{Y})^{2} \int_{\mathbf{w}, \mathbf{v}, \mathbf{x}, \mathbf{y}, \mathbf{z}, \mathbf{z}^{\prime}} \frac{X \cdot Y W^{\prime} \cdot V^{\prime}}{X^{2} Y^{2}\left(W^{\prime}\right)^{2}\left(V^{\prime}\right)^{2}} \rho^{b}(\mathbf{w}) \rho^{b}(\mathbf{v}) \rho^{a}(\mathbf{x}) \rho^{a}(\mathbf{y}) .
\end{aligned}
$$

where $K_{\mathrm{JSJ}}(\mathbf{x}, \mathbf{y}, \mathbf{z})$ is defined in (2.66). The first non-trivial term is a LO contribution, which is of order $\alpha_{s}$. The second term is an $\alpha_{s}^{2}$ correction and, because of its linear dependence on $\delta Y$, it will be contributing to virtual terms in the NLO JIMWLK Hamiltonian. The last term will contribute to subtraction of $(\mathrm{LO})^{2}$ terms in the evolution.

The phase of the LCWF is computed in appendix F from the condition (3.31):

$$
i \phi^{\mathrm{NLO}}=-\delta \mathrm{Y} \int_{\mathbf{w}, \mathbf{x}, \mathbf{y}, \mathbf{z}, \mathbf{z}^{\prime}} K_{\mathrm{JJSSJ}}\left(\mathbf{w}, \mathbf{x}, \mathbf{y}, \mathbf{z}, \mathbf{z}^{\prime}\right) f^{a c b} \rho^{a}(\mathbf{x}) \rho^{b}(\mathbf{y}) \rho^{c}(\mathbf{w}),
$$

where $K_{\text {JJSSJ }}$ is defined in (2.57). Up to order $\alpha_{s}^{2}$,

$$
\mathcal{N}^{\mathrm{NLO}}=\left\|\mathcal{N}^{\mathrm{NLO}}\right\|+i \phi^{\mathrm{NLO}}
$$

\section{The next to leading order JIMWLK Hamiltonian}

The aim of this section is to compute $\Sigma(1.3)$, that is the $\hat{S}$-matrix expectation value in the LCWF (3.84), which was calculated in the previous section. Each soft quark or gluon in the LCWF will contribute one eikonal factor $S$ defined respectively in (2.38) and (2.37). The $\rho$ factors in the ket/bra states will be converted into left/right rotation operators $J_{L / R}$ according to (2.39). There are nine overlaps that emerge in this computation, which we classify according to the number of $J$ operators and the Wilson lines $S$ that they contain:

$$
\begin{aligned}
\Sigma & =\left\langle\psi^{\mathrm{NLO}}|\hat{S}-1| \psi^{\mathrm{NLO}}\right\rangle \\
& =\Sigma^{L O}+\Sigma_{q \bar{q}}+\Sigma_{\text {JJSSJ }}+\Sigma_{\text {JSSJ }}+\Sigma_{\text {JJSJ }}+\Sigma_{\text {JSJ }}+\Sigma_{\text {JJSSJJ }}+\Sigma_{\text {JJJSJ }}+\Sigma_{\text {virtual }} .
\end{aligned}
$$

$\Sigma^{L O}$ was defined in (2.54). The other entrees (all of order $\alpha_{s}^{2}$ ) are defined below:

$$
\begin{aligned}
\Sigma_{q \bar{q}} & \equiv\left\langle\psi_{q \bar{q} \rho}|\hat{S}| \psi_{q \bar{q} \rho}\right\rangle, \\
\Sigma_{\text {JJSSJ }} & \equiv\left\langle\psi_{g g \rho}|\hat{S}| \psi_{g g \rho \rho}\right\rangle+\left\langle\psi_{g g \rho \rho}|\hat{S}| \psi_{g g \rho}\right\rangle, \\
\Sigma_{\text {JSSJ }} & \equiv\left\langle\psi_{g g}|\hat{S}| \psi_{g g \rho}\right\rangle, \\
\Sigma_{\text {JJSJ }} & \equiv\left\langle\psi_{g \rho \rho}|\hat{S}| \psi_{g \rho}^{L O}\right\rangle+\left\langle\psi_{g \rho}^{L O}|\hat{S}| \psi_{g \rho \rho}\right\rangle,
\end{aligned}
$$




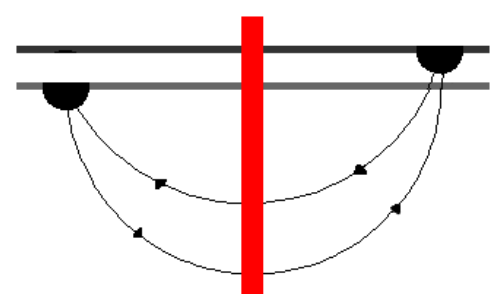

(a)

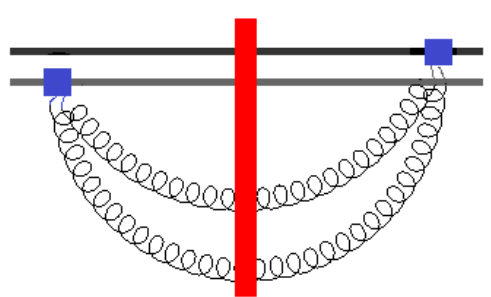

(b)

Figure 10. (a) - The diagram for $\Sigma_{q \bar{q}}$, (b) - The diagram for $\Sigma_{\text {JSSJ }}$. The black blub denotes the effective emission vertex, which is defined by $\left|\psi_{q \bar{q} \rho}\right\rangle$. The effective two gluon emission vertex corresponds to $\left.\left|\psi_{g g}\right\rangle\right\rangle$.

$$
\begin{aligned}
\Sigma_{\text {JSJ }} & \equiv\left\langle\psi_{g \rho}|\hat{S}| \psi_{g \rho}^{L O}\right\rangle+\left\langle\psi_{g \rho}^{L O}|\hat{S}| \psi_{g \rho}\right\rangle, \\
\Sigma_{\text {JJSSJJ }} & \equiv\left\langle\psi_{g g \rho \rho}|\hat{S}| \psi_{g g \rho \rho}\right\rangle, \\
\Sigma_{\text {JJJSJ }} & \equiv\left\langle\psi_{g \rho \rho \rho}|\hat{S}| \psi_{g \rho}^{L O}\right\rangle+\left\langle\psi_{g \rho}^{L O}|\hat{S}| \psi_{g \rho \rho \rho}\right\rangle, \\
\Sigma_{\text {virtual }} & \equiv\left\langle 0\left|\left(\mathcal{N}^{\mathrm{NLO}}\right)^{\dagger} \hat{S} \mathcal{N}^{\mathrm{NLO}}\right| 0\right\rangle-1 .
\end{aligned}
$$

For each of these contributions a dedicated subsection will be devoted. Some details of the calculations are provided in appendix $\mathrm{H}$.

As anticipated, we will find not only contributions linear in $\delta \mathrm{Y}$, but also contributions of order $(\delta \mathrm{Y})^{2}$. To make separation between these very different types of contributions crystal clear, we will introduce an additional label (upper script) - NLO or $(\delta \mathrm{Y})^{2}$ respectively. Each $\Sigma_{\ldots}$ defined in (4.2)-(4.9) is decomposed according to:

$$
\Sigma_{\ldots}=\Sigma_{\ldots}^{\mathrm{NLO}}\left(\alpha_{s}^{2}, \delta \mathrm{Y}\right)+\Sigma_{\ldots}^{(\delta \mathrm{Y})^{2}}\left(\alpha_{s}^{2},(\delta \mathrm{Y})^{2}\right) .
$$

At the end, in section 4.8, we will assemble together all the contributions at NLO and write down the NLO JIMWLK Hamiltonian defined in (1.6).

Finally, in section 4.9 we will collect all the $(\delta \mathrm{Y})^{2}$ contributions, and show that they correspond to contributions which are generated from the LO Hamiltonian applied twice. Based on the expansion (1.4), we will show that the anticipated relation:

$$
\Sigma^{(\delta \mathrm{Y})^{2}}=\frac{1}{2}\left(\delta Y H_{\mathrm{JIMWLK}}^{\mathrm{LO}}\right)^{2}
$$

holds indeed. This relation serves as a strong consistency check on our calculation.

\subsection{Computation of $\Sigma_{q \bar{q}}$}

$\Sigma_{q \bar{q}}$ is defined in (4.2) as an overlap between the incoming state $\left|\psi_{q \bar{q} \rho}\right\rangle$ (3.90), before and after passing through the shockwave (figure 10a).

Representing the operators $\rho$ in terms of the operators $J_{L}$ and $J_{R}$ as explained in section 2.6, and replacing the products of Pauli matrices using the identity $\sigma^{i} \sigma^{j}=i \varepsilon^{i j k} \sigma^{k}+\delta^{i j} I$,

$$
\begin{aligned}
\Sigma_{q \bar{q}}= & \int_{\mathbf{x}, \mathbf{y}, \mathbf{z}, \mathbf{z}^{\prime}} \int_{\Lambda}^{e^{\delta \curlyvee} \Lambda} d k^{+} \int_{0}^{1} d \xi \int d^{2} \mathbf{k} d^{2} \mathbf{p} d^{2} \mathbf{u} d^{2} \mathbf{v} e^{-i \mathbf{v} \cdot Z+i \mathbf{u} \cdot Y^{\prime}+i \mathbf{p} \cdot Z-i \mathbf{k} \cdot X^{\prime}} \\
& \times \frac{g^{4} N_{f} J_{L}^{a}(\mathbf{x}) \operatorname{Tr}\left[S^{\dagger}(\mathbf{z}) t^{a} S\left(\mathbf{z}^{\prime}\right) t^{b}\right] J_{R}^{b}(\mathbf{y})}{512 \pi^{10}\left((1-\xi) \mathbf{p}^{2}+\xi(\mathbf{k}-\mathbf{p})^{2}\right)\left((1-\xi) \mathbf{v}^{2}+\xi(\mathbf{u}-\mathbf{v})^{2}\right) k^{+}}
\end{aligned}
$$




$$
\begin{aligned}
& \times\left(\frac{(1-2 \xi)^{2}\left(\mathbf{p}^{i}-\xi \mathbf{k}^{i}\right)\left(\mathbf{v}^{j}-\xi \mathbf{u}^{j}\right)+\varepsilon^{i m} \varepsilon^{j s}\left(\mathbf{p}^{m}-\xi \mathbf{k}^{m}\right)\left(\mathbf{v}^{s}-\xi \mathbf{u}^{s}\right)}{\mathbf{k}^{2} \mathbf{u}^{2}} \mathbf{k}^{i} \mathbf{u}^{j}\right. \\
& \left.-2 \xi(1-\xi)(1-2 \xi)\left(\frac{\mathbf{k} \cdot(\xi \mathbf{k}-\mathbf{p})}{\mathbf{k}^{2}}+\frac{\mathbf{u} \cdot(\xi \mathbf{u}-\mathbf{v})}{\mathbf{u}^{2}}\right)+4 \xi^{2}(1-\xi)^{2}\right)
\end{aligned}
$$

We have used the fact that the scattering matrix is diagonal in coordinate space:

$$
\begin{aligned}
& \left\langle\bar{q}_{\lambda_{4}}^{\delta, \bar{f}}(u-v) q_{\lambda_{3}}^{\gamma, \bar{f}}(v)|\hat{S}| \bar{q}_{\lambda_{2}}^{\beta, f}(k-p) q_{\lambda_{1}}^{\alpha, f}(p)\right\rangle \\
& \quad=\frac{N_{f} \delta_{\lambda_{1} \lambda_{3}} \delta_{\lambda_{2} \lambda_{4}}}{(2 \pi)^{4} k^{+}} \int_{\mathbf{z}, \mathbf{z}^{\prime}} S^{\delta \alpha}(\mathbf{z}) S^{\dagger \beta \gamma}\left(\mathbf{z}^{\prime}\right) e^{-i \mathbf{v} \cdot\left(\mathbf{z}-\mathbf{z}^{\prime}\right)+i \mathbf{p} \cdot\left(\mathbf{z}-\mathbf{z}^{\prime}\right)-i \mathbf{u} \cdot \mathbf{z}^{\prime}+i \mathbf{k} \cdot \mathbf{z}^{\prime}} \delta\left(u^{+}-k^{+}\right) \delta(\xi-\vartheta),
\end{aligned}
$$

Here $\xi \equiv \frac{p^{+}}{k^{+}}$and $\vartheta \equiv \frac{v^{+}}{u^{+}}$. Equation (4.12) coincides with eq. (14) of [52, 53], after the identity (G.5) and a shift in momenta are applied. The rest of the computation can be found in appendix H.1. After integration over $\xi$ in (H.5), using (C.2), (C.3), and (C.4),

$$
\begin{aligned}
& \Sigma_{q \bar{q}}=\delta \mathrm{Y} \int_{\mathbf{x}, \mathbf{y}, \mathbf{z}, \mathbf{z}^{\prime}} \frac{g^{4} N_{f} J_{L}^{a}(\mathbf{x}) \operatorname{Tr}\left[S^{\dagger}(\mathbf{z}) t^{a} S\left(\mathbf{z}^{\prime}\right) t^{b}\right] J_{R}^{b}(\mathbf{y})}{64 \pi^{6} Z^{4}}\left(2-\frac{Z^{2}}{\left(X^{\prime}\right)^{2}-X^{2}} \ln \left(\frac{X^{2}}{\left(X^{\prime}\right)^{2}}\right)\right. \\
& -\frac{Z^{2}}{\left(Y^{\prime}\right)^{2}-Y^{2}} \ln \left(\frac{Y^{2}}{\left(Y^{\prime}\right)^{2}}\right)-\frac{2 X^{2}\left(X^{\prime}\right)^{2}\left(\left(Y^{\prime}\right)^{2}-Y^{2}\right)}{\left(\left(X^{\prime}\right)^{2}-X^{2}\right)\left(\left(X^{\prime}\right)^{2} Y^{2}-X^{2}\left(Y^{\prime}\right)^{2}\right)} \ln \left(\frac{X^{2}}{\left(X^{\prime}\right)^{2}}\right) \\
& \left.+\frac{2 Y^{2}\left(Y^{\prime}\right)^{2}\left(\left(X^{\prime}\right)^{2}-X^{2}\right)}{\left(\left(Y^{\prime}\right)^{2}-Y^{2}\right)\left(\left(X^{\prime}\right)^{2} Y^{2}-X^{2}\left(Y^{\prime}\right)^{2}\right)} \ln \left(\frac{Y^{2}}{\left(Y^{\prime}\right)^{2}}\right)-\frac{(X-Y)^{2} Z^{2}}{\left(X^{\prime}\right)^{2} Y^{2}-X^{2}\left(Y^{\prime}\right)^{2}} \ln \left(\frac{\left(X^{\prime}\right)^{2} Y^{2}}{X^{2}\left(Y^{\prime}\right)^{2}}\right)\right) .
\end{aligned}
$$

The result contains terms of order $\delta \mathrm{Y}$ only. To indicate this, we add the upper script $N L O$, so that $\Sigma_{q \bar{q}}^{\mathrm{NLO}} \equiv \Sigma_{q \bar{q}}$. (4.14) can be equivalently written as:

$$
\Sigma_{q \bar{q}}^{\mathrm{NLO}}=-\delta \mathrm{Y} \int_{\mathbf{x}, \mathbf{y}, \mathbf{z}, \mathbf{z}^{\prime}} K_{q \bar{q}}\left(\mathbf{x}, \mathbf{y}, \mathbf{z}, \mathbf{z}^{\prime}\right)\left[2 J_{L}^{a}(\mathbf{x}) \operatorname{tr}\left[S^{\dagger}(\mathbf{z}) t^{a} S\left(\mathbf{z}^{\prime}\right) t^{b}\right] J_{R}^{b}(\mathbf{y})\right],
$$

where $K_{q \bar{q}}\left(\mathbf{x}, \mathbf{y}, \mathbf{z}, \mathbf{z}^{\prime}\right)$ is defined in $(2.67)$.

\subsection{Computation of $\Sigma_{\text {JSSJ }}$}

$\Sigma_{\text {JSSJ }}$ is defined in (4.4) as an overlap of $\mid \psi_{g g} \rho$, , (3.94), before and after passing through the shockwave. The relevant diagrams appears in figure $10 \mathrm{~b}$.

$$
\begin{aligned}
\Sigma_{\mathrm{JSSJ}}= & \int_{\mathbf{x}, \mathbf{y}, \mathbf{z}, \mathbf{z}^{\prime}} \int_{\Lambda}^{e^{\delta \curlyvee} \Lambda} d k^{+} \int_{\frac{\Lambda}{k^{+}}}^{1-\frac{\Lambda}{k^{+}}} d \xi \frac{g^{4} f^{a b c} f^{d e f} J_{L}^{a}(\mathbf{x}) S_{A}^{b e}(\mathbf{z}) S_{A}^{c f}\left(\mathbf{z}^{\prime}\right) J_{R}^{d}(\mathbf{y}) \xi(1-\xi)}{32 \pi^{6} k^{+}\left((1-\xi)\left(X^{\prime}\right)^{2}+\xi X^{2}\right)\left((1-\xi)\left(Y^{\prime}\right)^{2}+\xi Y^{2}\right)} \\
& \times\left(\delta^{j l} \frac{X^{2}-\left(X^{\prime}\right)^{2}}{2 Z^{2}}+\frac{1}{\xi}\left[\frac{\left(X^{\prime}\right)^{j} Z^{l}}{Z^{2}}+\frac{X^{l}\left(X^{\prime}\right)^{j}}{2 X^{2}}\right]+\frac{1}{1-\xi}\left[\frac{X^{l} Z^{j}}{Z^{2}}-\frac{X^{l}\left(X^{\prime}\right)^{j}}{2\left(X^{\prime}\right)^{2}}\right]\right) \\
& \times\left(\delta^{j l} \frac{Y^{2}-\left(Y^{\prime}\right)^{2}}{2 Z^{2}}+\frac{1}{\xi}\left[\frac{\left(Y^{\prime}\right)^{j} Z^{l}}{Z^{2}}+\frac{Y^{l}\left(Y^{\prime}\right)^{j}}{2 Y^{2}}\right]+\frac{1}{1-\xi}\left[\frac{Y^{l} Z^{j}}{Z^{2}}-\frac{Y^{l}\left(Y^{\prime}\right)^{j}}{2\left(Y^{\prime}\right)^{2}}\right]\right) .
\end{aligned}
$$

where the following matrix element was used:

$$
\begin{aligned}
\left\langle g_{m}^{e}\left(u^{+}-v^{+}, \mathbf{z}^{\prime}\right) g_{n}^{f}\left(v^{+}, \mathbf{z}\right)|\hat{S}| g_{j}^{b}\left(k^{+}-p^{+}, \overline{\mathbf{z}}^{\prime}\right) g_{l}^{c}\left(p^{+}, \overline{\mathbf{z}}\right)\right\rangle \\
=\delta\left(u^{+}-k^{+}\right)\left(S_{A}^{b f}(\mathbf{z}) S_{A}^{c e}\left(\mathbf{z}^{\prime}\right) \delta\left(k^{+}-p^{+}-v^{+}\right) \delta^{(2)}\left(\mathbf{z}-\overline{\mathbf{z}}^{\prime}\right) \delta^{(2)}\left(\mathbf{z}^{\prime}-\overline{\mathbf{z}}\right) \delta_{n j} \delta_{l m}\right. \\
\left.\quad+S_{A}^{b e}(\mathbf{z}) S_{A}^{c f}\left(\mathbf{z}^{\prime}\right) \delta\left(p^{+}-v^{+}\right) \delta^{(2)}\left(\mathbf{z}^{\prime}-\overline{\mathbf{z}}^{\prime}\right) \delta^{(2)}(\mathbf{z}-\overline{\mathbf{z}}) \delta_{m j} \delta_{n l}\right) .
\end{aligned}
$$



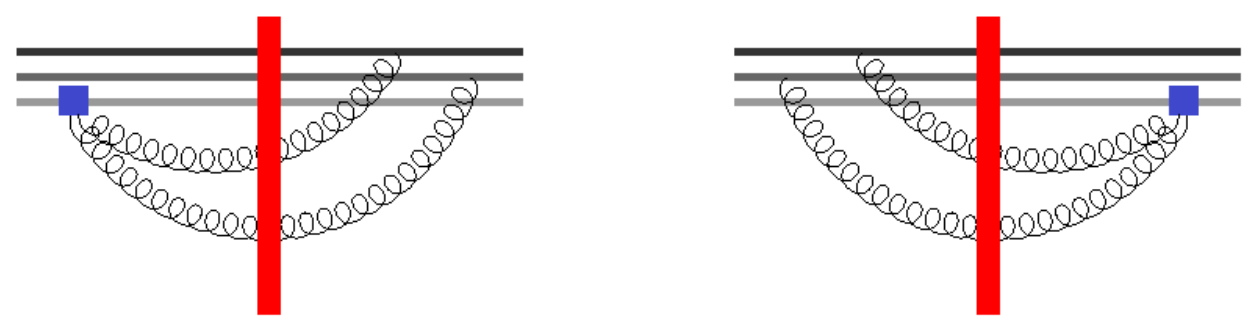

Figure 11. The diagrams for $\Sigma_{\text {JJSSJ }}$. Effective two gluon emission vertex corresponds to $\left|\psi_{\text {gg } \rho}\right\rangle$.

The remaining computation is moved to appendix H.2. The result is:

$$
\Sigma_{\mathrm{JSSJ}}=\Sigma_{\mathrm{JSSJ}}^{\mathrm{NLO}}+\Sigma_{\mathrm{JSSJ}}^{(\delta \mathrm{Y})^{2}}
$$

with

$$
\sum_{\mathrm{JSSJ}}^{\mathrm{NLO}}=-\delta \mathrm{Y} \int_{\mathbf{x}, \mathbf{y}, \mathbf{z}, \mathbf{z}^{\prime}} K_{\mathrm{JSSJ}}\left(\mathbf{x}, \mathbf{y}, \mathbf{z}, \mathbf{z}^{\prime}\right) f^{a b c} f^{d e f} S_{A}^{b e}(\mathbf{z}) S_{A}^{c f}\left(\mathbf{z}^{\prime}\right) J_{L}^{a}(\mathbf{x}) J_{R}^{d}(\mathbf{y}),
$$

where $K_{\text {JSSJ }}\left(\mathbf{x}, \mathbf{y}, \mathbf{z}, \mathbf{z}^{\prime}\right)$ is defined in (2.59), and

$$
\begin{aligned}
\Sigma_{\mathrm{JSSS}}^{(\delta Y)^{2}}= & \frac{\alpha_{s}^{2}}{4 \pi^{4}}(\delta \mathbf{Y})^{2} \int_{\mathbf{x}, \mathbf{y}, \mathbf{z}, \mathbf{z}^{\prime}}\left(\frac{2 X \cdot Y}{Z^{2} X^{2} Y^{2}}-\frac{X \cdot Y Z \cdot Y^{\prime}}{X^{2} Y^{2} Z^{2}\left(Y^{\prime}\right)^{2}}-\frac{X \cdot Y X^{\prime} \cdot Z}{X^{2} Y^{2}\left(X^{\prime}\right)^{2} Z^{2}}\right. \\
& \left.+\frac{X \cdot Y X^{\prime} \cdot Y^{\prime}}{2 X^{2} Y^{2}\left(X^{\prime}\right)^{2}\left(Y^{\prime}\right)^{2}}\right) f^{a b c} f^{d e f} S_{A}^{b e}(\mathbf{z}) S_{A}^{c f}\left(\mathbf{z}^{\prime}\right) J_{L}^{a}(\mathbf{x}) J_{R}^{d}(\mathbf{y}) .
\end{aligned}
$$

\subsection{Computation of $\Sigma_{\text {JJSSJ }}$}

$\Sigma_{\text {JJSSJ }}$ is defined in (4.3) as an $S$-matrix overlap between $\left|\psi_{\text {gg } \rho}\right\rangle$ (3.94) and $\left|\psi_{\text {gg } \rho \rho}\right\rangle$ (3.96). The relevant diagrams appear in figure 11. With the aid of (4.17),

$$
\begin{aligned}
\Sigma_{\mathrm{JJSSJ}}= & \frac{i g^{4} f^{a c b}}{64 \pi^{6}} \int_{\mathbf{w}, \mathbf{x}, \mathbf{y}, \mathbf{z}, \mathbf{z}^{\prime}} \int_{\Lambda}^{e^{\delta \curlyvee} \Lambda} \frac{d k^{+}}{k^{+}} \int_{\frac{\Lambda}{k^{+}}}^{1-\frac{\Lambda}{k^{+}}} d \xi \\
& \times \frac{1}{\left((1-\xi)\left(W^{\prime}\right)^{2}+\xi W^{2}\right)}\left(\frac{\left(Y^{\prime}\right)^{j} X^{i}}{\left(Y^{\prime}\right)^{2} X^{2}}-\frac{Y^{i}\left(X^{\prime}\right)^{j}}{\left(X^{\prime}\right)^{2} Y^{2}}\right) \\
& \times\left(\frac{\delta^{i j}}{2 Z^{2}}\left(W^{2}-\left(W^{\prime}\right)^{2}\right)-\frac{\left(W^{\prime}\right)^{j} Z^{i}}{\xi Z^{2}}+\frac{W^{i} Z^{j}}{(1-\xi) Z^{2}}+\frac{W^{i}\left(W^{\prime}\right)^{j}}{2 \xi W^{2}}-\frac{W^{i}\left(W^{\prime}\right)^{j}}{2(1-\xi)\left(W^{\prime}\right)^{2}}\right) \\
& \times\left[J_{L}^{d}(\mathbf{x}) J_{L}^{e}(\mathbf{y}) S_{A}^{d c}(\mathbf{z}) S_{A}^{e b}\left(\mathbf{z}^{\prime}\right) J_{R}^{a}(\mathbf{w})-J_{L}^{a}(\mathbf{w}) S_{A}^{c d}(\mathbf{z}) S_{A}^{b e}\left(\mathbf{z}^{\prime}\right) J_{R}^{d}(\mathbf{x}) J_{R}^{e}(\mathbf{y})\right],
\end{aligned}
$$

Notice that the minus sign between the terms in the last line in (4.21) is due to complex conjugation. The integration over $\xi$ is done using (C.2), (C.5) and (C.6):

$$
\begin{aligned}
& \Sigma_{\text {JJSSJ }}=\frac{i g^{4} f^{a c b}}{64 \pi^{6}} \int_{\mathbf{w}, \mathbf{x}, \mathbf{y}, \mathbf{z}, \mathbf{z}^{\prime}} \int_{\Lambda}^{e^{\delta \curlyvee} \Lambda} \frac{d k^{+}}{k^{+}}\left(\frac{\left(Y^{\prime}\right)^{j} X^{i}}{\left(Y^{\prime}\right)^{2} X^{2}}-\frac{Y^{i}\left(X^{\prime}\right)^{j}}{\left(X^{\prime}\right)^{2} Y^{2}}\right) \\
& \times\left[\left(\frac{\delta^{i j}}{2 Z^{2}}-\frac{\left(W^{\prime}\right)^{j} Z^{i}}{\left(W^{\prime}\right)^{2} Z^{2}}+\frac{W^{i} Z^{j}}{W^{2} Z^{2}}-\frac{W^{i}\left(W^{\prime}\right)^{j}}{W^{2}\left(W^{\prime}\right)^{2}}\right) \ln \left(\frac{W^{2}}{\left(W^{\prime}\right)^{2}}\right)-\left(\frac{\left(W^{\prime}\right)^{j} Z^{i}}{\left(W^{\prime}\right)^{2} Z^{2}}+\frac{W^{i} Z^{j}}{W^{2} Z^{2}}\right)\right. \\
& \left.\quad \times \ln \left(\frac{\Lambda}{k^{+}}\right)\right]\left[J_{L}^{d}(\mathbf{x}) J_{L}^{e}(\mathbf{y}) S_{A}^{d c}(\mathbf{z}) S_{A}^{e b}\left(\mathbf{z}^{\prime}\right) J_{R}^{a}(\mathbf{w})-J_{L}^{a}(\mathbf{w}) S_{A}^{c d}(\mathbf{z}) S_{A}^{b e}\left(\mathbf{z}^{\prime}\right) J_{R}^{d}(\mathbf{x}) J_{R}^{e}(\mathbf{y})\right] .
\end{aligned}
$$



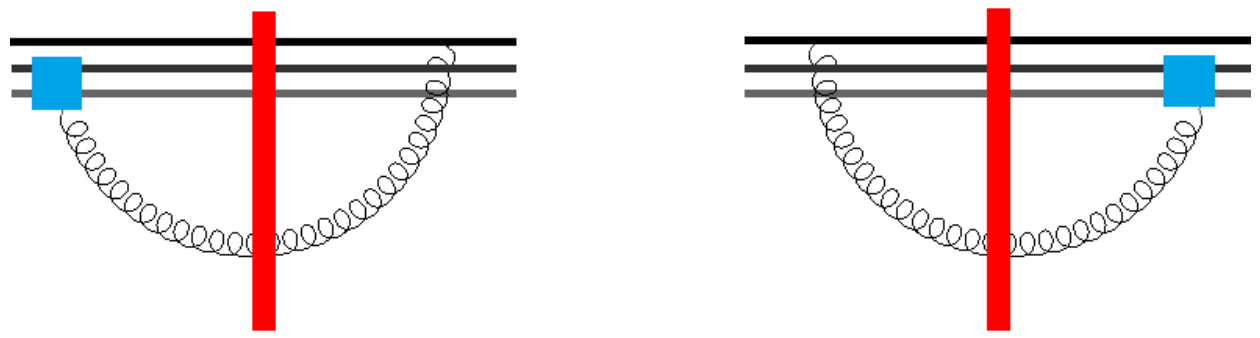

Figure 12. The diagrams for $\Sigma_{\text {JJSJ }}$. Effective one gluon emission vertex corresponds to $\left|\psi_{g \rho \rho}\right\rangle$.

It is important to notice that $J_{L}$ and $J_{R}$ in (4.22) by construction do not act on $S_{A}$ inside (4.22). As anticipated, the result contains both terms proportional to $\delta \mathrm{Y}$ and $(\delta \mathrm{Y})^{2}$, which are split as:

$$
\Sigma_{\mathrm{JJSSJ}}=\Sigma_{\mathrm{JJSSJ}}^{\mathrm{NLO}}+\Sigma_{\mathrm{JJSSJ}}^{(\delta)^{2}}
$$

$\Sigma_{\text {JJSSJ }}^{(\delta Y)^{2}}$ emerges from the term $\ln \left(\frac{\Lambda}{k^{+}}\right)$, which becomes proportional to $(\delta \mathrm{Y})^{2}$ after integration over $k^{+} . \Sigma_{\text {JJSSJ }}^{\mathrm{NLO}}$ reads:

$$
\begin{aligned}
& \sum_{\mathrm{JJSSJ}}^{\mathrm{NLO}}=-\delta \mathrm{Y} \int_{\mathbf{w}, \mathbf{x}, \mathbf{y}, \mathbf{z}, \mathbf{z}^{\prime}} K_{\mathrm{JJSSJ}}\left(\mathbf{w}, \mathbf{x}, \mathbf{y}, \mathbf{z}, \mathbf{z}^{\prime}\right) \\
& \quad \times f^{a c b}\left[J_{L}^{d}(\mathbf{x}) J_{L}^{e}(\mathbf{y}) S_{A}^{d c}(\mathbf{z}) S_{A}^{e b}\left(\mathbf{z}^{\prime}\right) J_{R}^{a}(\mathbf{w})-J_{L}^{a}(\mathbf{w}) S_{A}^{c d}(\mathbf{z}) S_{A}^{b e}\left(\mathbf{z}^{\prime}\right) J_{R}^{d}(\mathbf{x}) J_{R}^{e}(\mathbf{y})\right] .
\end{aligned}
$$

where $K_{\mathrm{JJSSJ}}\left(\mathbf{w}, \mathbf{x}, \mathbf{y}, \mathbf{z}, \mathbf{z}^{\prime}\right)$ is defined in (2.57).

$$
\begin{aligned}
& \Sigma_{\text {JJSSJ }}^{(\delta \mathbf{Y})^{2}}=\frac{i \alpha_{s}^{2}}{8 \pi^{4}}(\delta \mathbf{Y})^{2} \int_{\mathbf{w}, \mathbf{x}, \mathbf{y}, \mathbf{z}, \mathbf{z}^{\prime}}\left(\frac{\left(Y^{\prime}\right)^{j} X^{i}}{\left(Y^{\prime}\right)^{2} X^{2}}-\frac{Y^{i}\left(X^{\prime}\right)^{j}}{\left(X^{\prime}\right)^{2} Y^{2}}\right)\left(\frac{\left(W^{\prime}\right)^{j} Z^{i}}{\left(W^{\prime}\right)^{2} Z^{2}}+\frac{W^{i} Z^{j}}{W^{2} Z^{2}}\right) \\
& \quad \times f^{a c b}\left[J_{L}^{d}(\mathbf{x}) J_{L}^{e}(\mathbf{y}) S_{A}^{d c}(\mathbf{z}) S_{A}^{e b}\left(\mathbf{z}^{\prime}\right) J_{R}^{a}(\mathbf{w})-J_{L}^{a}(\mathbf{w}) S_{A}^{c d}(\mathbf{z}) S_{A}^{b e}\left(\mathbf{z}^{\prime}\right) J_{R}^{d}(\mathbf{x}) J_{R}^{e}(\mathbf{y})\right] .
\end{aligned}
$$

\subsection{Computation of $\Sigma_{\text {JJSJ }}$}

$\Sigma_{\text {JJSJ }}$ is defined in (4.5) as an $S$-matrix element between the state $\left|\psi_{g \rho \rho}\right\rangle,(3.100)$, and the state $\left|\psi_{g \rho}^{L O}\right\rangle,(3.4)$. The relevant diagrams appear in figure 12.

$$
\begin{aligned}
& \Sigma_{\mathrm{JJSJ}}=-\frac{g^{4} f^{b d e}}{128 \pi^{7}} \int_{\Lambda}^{e^{\delta \curlyvee} \Lambda} \frac{d k^{+}}{k^{+}} \int_{\mathbf{w}, \mathbf{x}, \mathbf{y}, \mathbf{z}} \frac{W^{i}}{W^{2}}\left[J_{L}^{d}(\mathbf{x}) J_{L}^{e}(\mathbf{y}) S_{A}^{b a}(\mathbf{z}) J_{R}^{a}(\mathbf{w})\right. \\
& \left.-J_{L}^{a}(\mathbf{w}) S_{A}^{a b}(\mathbf{z}) J_{R}^{d}(\mathbf{x}) J_{R}^{e}(\mathbf{y})\right] \int d^{2} \mathbf{k} d^{2} \mathbf{p} e^{-i \mathbf{k} \cdot X+i \mathbf{p} \cdot(X-Y)} \frac{1}{\mathbf{p}^{2} \mathbf{k}^{2}} \\
& \left\{\left(\left[\left(2-\frac{\mathbf{k} \cdot \mathbf{p}}{(\mathbf{k}-\mathbf{p})^{2}}\right) \ln \left(\frac{\Lambda}{k^{+}}\right)+\frac{2 \mathbf{p}^{2}}{(\mathbf{k}-\mathbf{p})^{2}} \ln \left(\frac{(\mathbf{k}-\mathbf{p})^{2}}{\mathbf{k}^{2}}\right)\right] \mathbf{k}^{i}+\ln \left(\frac{\Lambda}{k^{+}}\right) \frac{\mathbf{k}^{2}}{(\mathbf{k}-\mathbf{p})^{2}} \mathbf{p}^{i}\right)\right. \\
& \left.-\int_{\frac{\Lambda}{k^{+}}}^{1-\frac{\Lambda}{k^{+}}} d \xi \frac{\left[2\left(\mathbf{p}^{2}-\mathbf{k} \cdot \mathbf{p}\right)-\xi \mathbf{p}^{2}+2 \xi \mathbf{k} \cdot \mathbf{p}\right] \mathbf{k}^{i}+\mathbf{k}^{2} \mathbf{p}^{i}}{\left(\xi(\mathbf{k}-\mathbf{p})^{2}+(1-\xi) \mathbf{k}^{2}\right)(1-\xi)}\right\} .
\end{aligned}
$$



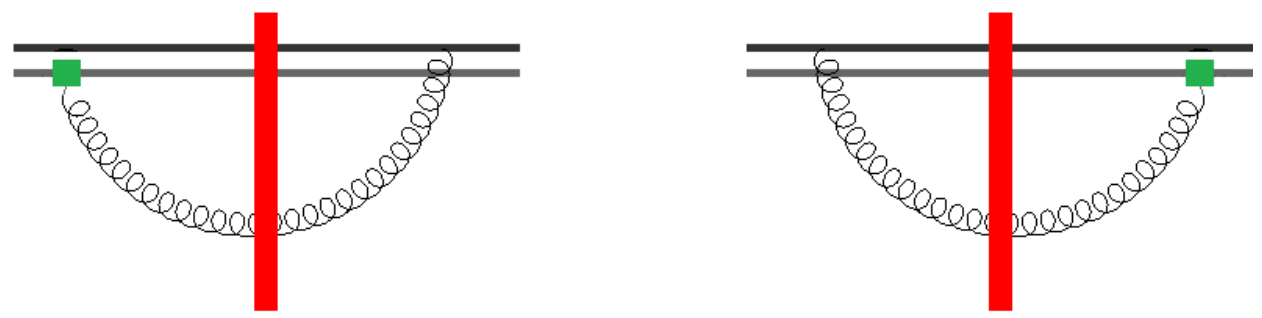

Figure 13. The diagrams for $\left.\Sigma_{\text {JSJ. Effective one gluon emission vertex corresponds to } \mid \psi_{g} \rho}\right\rangle$.

After the $\xi$ integral is evaluated using (C.18) and (C.19):

$$
\begin{aligned}
& \Sigma_{\mathrm{JJSJ}}=-\frac{g^{4} f^{b d e}}{128 \pi^{7}} \int_{\Lambda}^{e^{\delta \curlyvee} \Lambda} \frac{d k^{+}}{k^{+}} \int_{\mathbf{w}, \mathbf{x}, \mathbf{y}, \mathbf{z}} \frac{W^{i}}{W^{2}}\left[J_{L}^{d}(\mathbf{x}) J_{L}^{e}(\mathbf{y}) S_{A}^{b a}(\mathbf{z}) J_{R}^{a}(\mathbf{w})\right. \\
&\left.-J_{L}^{a}(\mathbf{w}) S_{A}^{a b}(\mathbf{z}) J_{R}^{d}(\mathbf{x}) J_{R}^{e}(\mathbf{y})\right]\left(\int d ^ { 2 } \mathbf { k } d ^ { 2 } \mathbf { p } e ^ { - i \mathbf { k } \cdot X + i \mathbf { p } \cdot ( X - Y ) } \left[\left(\frac{\mathbf{p}^{i}}{\mathbf{p}^{2}(\mathbf{k}-\mathbf{p})^{2}}-\frac{\mathbf{k}^{i}}{\mathbf{k}^{2}(\mathbf{k}-\mathbf{p})^{2}}\right.\right.\right. \\
&\left.\left.+\frac{\mathbf{k}^{i}}{\mathbf{k}^{2} \mathbf{p}^{2}}\right) \ln \left(\frac{(\mathbf{k}-\mathbf{p})^{2}}{\mathbf{k}^{2}}\right)-\left(\frac{2 \mathbf{k}^{i}}{\mathbf{k}^{2} \mathbf{p}^{2}}+\frac{\mathbf{p} \cdot(\mathbf{p}-\mathbf{k}) \mathbf{k}^{i}}{\mathbf{k}^{2} \mathbf{p}^{2}(\mathbf{k}-\mathbf{p})^{2}}+\frac{2 \mathbf{p}^{i}}{\mathbf{p}^{2}(\mathbf{k}-\mathbf{p})^{2}}\right) \ln \left(\frac{k^{+}}{\Lambda}\right)\right] \\
&-(X \leftrightarrow Y)) .
\end{aligned}
$$

As usual, this expression can be split according to:

$$
\Sigma_{\mathrm{JJSJ}}=\Sigma_{\mathrm{JJSJ}}^{\mathrm{NLO}}+\Sigma_{\mathrm{JJSJ}}^{(\delta \mathrm{Y})^{2}}
$$

The remaining computation is available in appendix H.3. The results:

$$
\begin{aligned}
\Sigma_{\mathrm{JJSJ}}^{\mathrm{NLO}}= & -\delta \mathrm{Y} \int_{\mathbf{w}, \mathbf{x}, \mathbf{y}, \mathbf{z}} K_{\mathrm{JJSJ}}(\mathbf{w}, \mathbf{x}, \mathbf{y}, \mathbf{z}) f^{b d e}\left[J_{L}^{d}(\mathbf{x}) J_{L}^{e}(\mathbf{y}) S_{A}^{b a}(\mathbf{z}) J_{R}^{a}(\mathbf{w})\right. \\
& \left.-J_{L}^{a}(\mathbf{w}) S_{A}^{a b}(\mathbf{z}) J_{R}^{d}(\mathbf{x}) J_{R}^{e}(\mathbf{y})\right] .
\end{aligned}
$$

With $K_{\mathrm{JJSJ}}(\mathbf{w}, \mathbf{x}, \mathbf{y}, \mathbf{z})$ defined in (2.58). In addition:

$$
\begin{aligned}
& \Sigma_{\text {JJSJ }}^{(\delta \mathbf{Y})^{2}} \\
& =\frac{i \alpha_{s}^{2} f^{b d e}}{4 \pi^{4}}(\delta \mathbf{Y})^{2} \int_{\mathbf{w}, \mathbf{x}, \mathbf{y}, \mathbf{z}, \mathbf{z}^{\prime}}\left[J_{L}^{d}(\mathbf{x}) J_{L}^{e}(\mathbf{y}) S_{A}^{b a}\left(\mathbf{z}^{\prime}\right) J_{R}^{a}(\mathbf{w})-J_{L}^{a}(\mathbf{w}) S_{A}^{a b}\left(\mathbf{z}^{\prime}\right) J_{R}^{d}(\mathbf{x}) J_{R}^{e}(\mathbf{y})\right] \\
& \quad \times\left[\frac{X \cdot Y Y^{\prime} \cdot W^{\prime}}{X^{2} Y^{2}\left(Y^{\prime}\right)^{2}\left(W^{\prime}\right)^{2}}-\frac{X \cdot Y X^{\prime} \cdot W^{\prime}}{X^{2} Y^{2}\left(X^{\prime}\right)^{2}\left(W^{\prime}\right)^{2}}-\frac{X \cdot Z Y^{\prime} \cdot W^{\prime}}{X^{2} Z^{2}\left(Y^{\prime}\right)^{2}\left(W^{\prime}\right)^{2}}+\frac{Y \cdot Z X^{\prime} \cdot W^{\prime}}{Y^{2} Z^{2}\left(X^{\prime}\right)^{2}\left(W^{\prime}\right)^{2}}\right] .
\end{aligned}
$$

\subsection{Computation of $\Sigma_{\mathbf{J S J}}$}

$\Sigma_{\text {JSJ }}$ is defined in (4.6) as an $S$-matrix element between the state $\mid \psi_{g} \rho$, , (3.98), and the state $\left|\psi_{g \rho}^{L O}\right\rangle,(3.4)$. The relevant diagrams appears in figure 13.

$$
\begin{aligned}
& \Sigma_{\mathrm{JSJ}}=-\frac{g^{4}}{64 \pi^{5}} \int_{\mathbf{x}, \mathbf{y}, \mathbf{z}} \int_{\Lambda}^{e^{\delta \curlyvee} \Lambda} d k^{+} \frac{X \cdot Y}{X^{2} Y^{2} k^{+}}\left\{\left(\left[b-N_{c} \ln \left(\frac{k^{+}}{\Lambda}\right)-2 N_{c} \delta \mathrm{Y}\right]\right.\right. \\
& \left.\times\left[-2 \gamma-\ln \left(\frac{X^{2} \mu_{\overline{M S}}^{2}}{4}\right)\right]-N_{c} \ln ^{2}\left(\frac{\Lambda e^{\delta \mathrm{Y}}}{k^{+}}\right)+N_{c} \ln ^{2}\left(\frac{\Lambda}{k^{+}}\right)-\left(\frac{67}{9}-\frac{\pi^{2}}{3}\right) N_{c}+\frac{10}{9} N_{f}\right)
\end{aligned}
$$




$$
\begin{aligned}
& +\int_{\frac{\Lambda}{k^{+}}}^{1-\frac{\Lambda}{k^{+}}} d \xi \frac{N_{c}}{\xi(1-\xi)}((\xi-2) \ln (\xi)+(\xi+1) \ln (1-\xi) \\
& \left.\left.-(\xi-2)\left[-2 \gamma-\ln \left(\frac{X^{2} \mu_{\overline{M S}}^{2}}{4}\right)\right]\right)+(X \leftrightarrow Y)\right\} J_{L}^{a}(\mathbf{x}) S_{A}^{a b}(\mathbf{z}) J_{R}^{b}(\mathbf{y}) .
\end{aligned}
$$

Using (C.11), (C.13) and (C.15) we can integrate over $\xi$ :

$$
\begin{aligned}
& \int_{\frac{\Lambda}{k^{+}}}^{1-\frac{\Lambda}{k^{+}}} d \xi \frac{N_{c}}{\xi(1-\xi)}((\xi-2) \ln (\xi)+(\xi+1) \ln (1-\xi) \\
& \left.\quad-(\xi-2)\left[-2 \gamma-\ln \left(\frac{X^{2} \mu \frac{2}{M S}}{4}\right)\right]\right)=-3 N_{c} \ln \left(\frac{\Lambda}{k^{+}}\right)\left[-2 \gamma-\ln \left(\frac{X^{2} \mu \frac{2}{M S}}{4}\right)\right] .
\end{aligned}
$$

and obtain:

$$
\begin{aligned}
\Sigma_{\mathrm{JSJ}}= & -\frac{g^{4}}{32 \pi^{5}} \int_{\mathbf{x}, \mathbf{y}, \mathbf{z}} \int_{\Lambda}^{e^{\delta \curlyvee} \Lambda} d k^{+} \frac{X \cdot Y}{X^{2} Y^{2} k^{+}}\left(\left(b+2 N_{c} \ln \left(\frac{k^{+}}{\Lambda}\right)-2 N_{c} \delta \mathrm{Y}\right)\right. \\
& \times\left(-2 \gamma-\frac{1}{2} \ln \left(\frac{X^{2} \mu_{\overline{M S}}^{2}}{4}\right)-\frac{1}{2} \ln \left(\frac{Y^{2} \mu_{\overline{M S}}^{2}}{4}\right)\right)-N_{c} \ln ^{2}\left(\frac{\Lambda e^{\delta \mathrm{Y}}}{k^{+}}\right)+N_{c} \ln ^{2}\left(\frac{\Lambda}{k^{+}}\right) \\
& \left.-\left(\frac{67}{9}-\frac{\pi^{2}}{3}\right) N_{c}+\frac{10}{9} N_{f}\right) J_{L}^{a}(\mathbf{x}) S_{A}^{a b}(\mathbf{z}) J_{R}^{b}(\mathbf{y}) .
\end{aligned}
$$

Now, after integration over $k^{+}, \Sigma_{\text {JSJ }}$ is split according to:

$$
\Sigma_{\mathrm{JSJ}}=\Sigma_{\mathrm{JSJ}}^{\mathrm{NLO}}+\Sigma_{\mathrm{JSJ}}^{(\delta \mathrm{Y})^{2}}
$$

with

$$
\Sigma_{\mathrm{JSJ}}^{\mathrm{NLO}}=-\delta \mathrm{Y} \int_{\mathbf{x}, \mathbf{y}, \mathbf{z}} K_{\mathrm{JSJ}}^{\prime}(\mathbf{x}, \mathbf{y}, \mathbf{z})\left[-2 J_{L}^{a}(\mathbf{x}) S_{A}^{a b}(\mathbf{z}) J_{R}^{b}(\mathbf{y})\right]
$$

where

$$
\begin{aligned}
K_{\mathrm{JSJ}}^{\prime}(\mathbf{x}, \mathbf{y}, \mathbf{z}) \equiv & \frac{\alpha_{s}^{2} X \cdot Y}{4 \pi^{3} X^{2} Y^{2}}\left(b\left(\frac{1}{2} \ln \left(\frac{X^{2} \mu_{\overline{M S}}^{2}}{4}\right)+\frac{1}{2} \ln \left(\frac{Y^{2} \mu_{\overline{M S}}^{2}}{4}\right)+2 \gamma\right)\right. \\
& \left.+\left(\frac{67}{9}-\frac{\pi^{2}}{3}\right) N_{c}-\frac{10}{9} N_{f}\right) .
\end{aligned}
$$

The result for $K_{\text {JSJ }}^{\prime}$ can be identified (up to $2 \gamma-\ln 4$ ) in equation (87) of [47]. The relation between $K_{\mathrm{JSJ}}^{\prime}(\mathbf{x}, \mathbf{y}, \mathbf{z})$ and $K_{\mathrm{JSJ}}(\mathbf{x}, \mathbf{y}, \mathbf{z})$ is given below, in (4.58). Also,

$$
\begin{aligned}
\Sigma_{\mathrm{JSJ}}^{(\delta \mathbf{Y})^{2}}= & -\frac{\alpha_{s}^{2} N_{c}}{4 \pi^{3}}(\delta \mathbf{Y})^{2} \int_{\mathbf{x}, \mathbf{y}, \mathbf{z}} \frac{X \cdot Y}{X^{2} Y^{2}}\left[\frac{4}{\epsilon}+4 \gamma+\ln \left(\frac{X^{2} \mu_{\overline{M S}}^{2}}{4}\right)+\ln \left(\frac{Y^{2} \mu_{\overline{M S}}^{2}}{4}\right)\right] \\
& \times J_{L}^{a}(\mathbf{x}) S_{A}^{a b}(\mathbf{z}) J_{R}^{b}(\mathbf{y}),
\end{aligned}
$$

which can be equivalently written as:

$$
\begin{aligned}
\Sigma_{\mathrm{JSJ}}^{(\delta \mathrm{Y})^{2}}= & -\frac{\alpha_{s}^{2} N_{c}}{4 \pi^{4}}(\delta \mathrm{Y})^{2} \int_{\mathbf{x}, \mathbf{y}, \mathbf{z}, \mathbf{z}^{\prime}}\left(\frac{2 X \cdot Y}{Z^{2} X^{2} Y^{2}}-\frac{X \cdot Y X^{\prime} \cdot Z}{X^{2} Y^{2}\left(X^{\prime}\right)^{2} Z^{2}}-\frac{X \cdot Y Y^{\prime} \cdot Z}{X^{2} Y^{2}\left(Y^{\prime}\right)^{2} Z^{2}}\right) \\
& \times J_{L}^{a}(\mathbf{x}) S_{A}^{a b}(\mathbf{z}) J_{R}^{b}(\mathbf{y}) .
\end{aligned}
$$



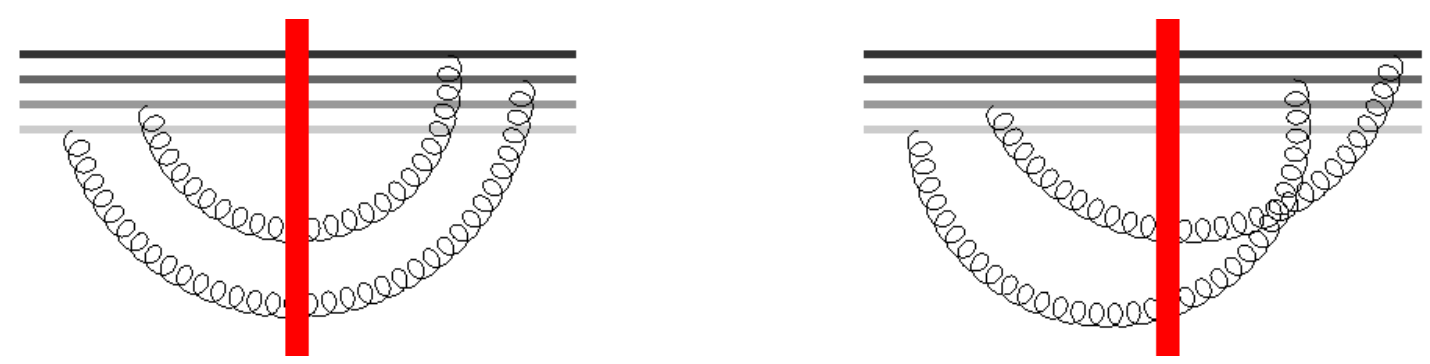

Figure 14. The diagrams for $\Sigma_{\text {JJSSJJ }}$.
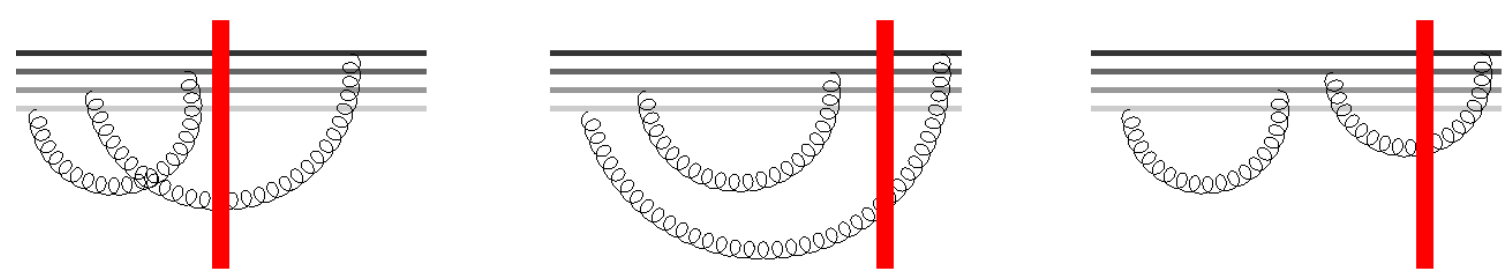

Figure 15. The diagrams for $\Sigma_{\text {JJJSJ }}$.

\subsection{Computation of $\Sigma_{\text {JJSSJJ }}$ and $\Sigma_{\text {JJJSJ }}$}

$\Sigma_{\text {JJSSJJ }}$ is defined in (4.7). This contribution is driven by $\left|\psi_{g g} \rho \rho\right\rangle$, (3.96), interfering with itself before and after passing though the shockwave, as shown in figure 14.

$$
\begin{aligned}
\Sigma_{\text {JJSSJJ }}= & \int_{\mathbf{w}, \mathbf{v}, \mathbf{x}, \mathbf{y}, \mathbf{z}, \mathbf{z}^{\prime}, \overline{\mathbf{z}}, \overline{\mathbf{z}}^{\prime}} \int_{\Lambda}^{e^{{ }^{\curlyvee} \Lambda} d k^{+} d \bar{k}^{+} \int_{\bar{k}^{+}}^{1-\frac{\Lambda}{k^{+}}} d \xi d \vartheta} \\
& \times \frac{g^{4} X^{i}\left(W^{\prime}\right)^{j} \bar{Y}^{k} \cdot\left(\bar{V}^{\prime}\right)^{l}}{256 \pi^{6} \sqrt{\xi(1-\xi) \vartheta(1-\vartheta)} X^{2} \bar{Y}^{2}\left(W^{\prime}\right)^{2}\left(\bar{V}^{\prime}\right)^{2}}\left\langle g_{l}^{c}\left((1-\vartheta) \bar{k}^{+}, \overline{\mathbf{z}}^{\prime}\right) g_{k}^{d}\left(\vartheta \bar{k}^{+}, \overline{\mathbf{z}}\right)\right| \\
& \times\left\{\rho^{c}(\mathbf{v}), \rho^{d}(\mathbf{y})\right\} \hat{S}\left\{\rho^{b}(\mathbf{w}), \rho^{a}(\mathbf{x})\right\}\left|g_{j}^{b}\left((1-\xi) k^{+}, \mathbf{z}^{\prime}\right) g_{i}^{a}\left(\xi k^{+}, \mathbf{z}\right)\right\rangle
\end{aligned}
$$

where $\bar{Y}=\mathbf{y}-\overline{\mathbf{z}}$ and $\bar{V}^{\prime}=\mathbf{v}-\overline{\mathbf{z}}^{\prime}$. The rest of this calculation is available in appendix H.4. The result after integration over $k^{+}$is:

$$
\begin{aligned}
\Sigma_{\mathrm{JJSSJJ}}^{(\delta \mathbf{Y})^{2}}= & \frac{\alpha_{s}^{2}}{2 \pi^{4}}(\delta \mathbf{Y})^{2} \int_{\mathbf{w}, \mathbf{v}, \mathbf{x}, \mathbf{y}, \mathbf{z}, \mathbf{z}^{\prime}} \frac{X \cdot Y W^{\prime} \cdot V^{\prime}}{X^{2} Y^{2}\left(W^{\prime}\right)^{2}\left(V^{\prime}\right)^{2}} J_{L}^{a}(\mathbf{x}) J_{L}^{b}(\mathbf{w}) S_{A}^{a d}(\mathbf{z}) S_{A}^{b c}\left(\mathbf{z}^{\prime}\right) J_{R}^{d}(\mathbf{y}) J_{R}^{c}(\mathbf{v}) \\
& -\frac{\alpha_{s}^{2}}{8 \pi^{4}}(\delta \mathbf{Y})^{2} \int_{\mathbf{x}, \mathbf{y}, \mathbf{z}, \mathbf{z}^{\prime}} \frac{X \cdot Y X^{\prime} \cdot Y^{\prime}}{X^{2} Y^{2}\left(X^{\prime}\right)^{2}\left(Y^{\prime}\right)^{2}} f^{a b c} f^{d e f} S_{A}^{b e}(\mathbf{z}) S_{A}^{c f}\left(\mathbf{z}^{\prime}\right) J_{L}^{a}(\mathbf{x}) J_{R}^{d}(\mathbf{y}) .
\end{aligned}
$$

Now let us compute $\Sigma_{\text {JJJSJ }}$ as defined in (4.8). The contribution comes from the $S$-matrix element between the state $\left|\psi_{g \rho \rho \rho}\right\rangle$ (3.103) and the state $\left|\psi_{g \rho}^{L O}\right\rangle(3.4)$, as shown in figure 15.

$$
\begin{aligned}
\Sigma_{\mathrm{JJJSJ}}= & -\frac{\alpha_{s}^{2}}{2 \pi^{4}}(\delta \mathbf{Y})^{2} \int_{\mathbf{w}, \mathbf{v}, \mathbf{x}, \mathbf{y}, \mathbf{z}, \mathbf{z}^{\prime}} \frac{X \cdot Y W^{\prime} \cdot V^{\prime}}{X^{2} Y^{2}\left(W^{\prime}\right)^{2}\left(V^{\prime}\right)^{2}} \\
& \times\left[S_{A}^{c d}\left(\mathbf{z}^{\prime}\right) J_{L}^{a}(\mathbf{x}) J_{L}^{a}(\mathbf{y}) J_{L}^{c}(\mathbf{w}) J_{R}^{d}(\mathbf{v})+S_{A}^{c d}\left(\mathbf{z}^{\prime}\right) J_{L}^{c}(\mathbf{v}) J_{R}^{a}(\mathbf{x}) J_{R}^{a}(\mathbf{y}) J_{R}^{d}(\mathbf{w})\right] .
\end{aligned}
$$


Which we write as:

$$
\begin{aligned}
\Sigma_{\mathrm{JJJSJ}}= & -\frac{\alpha_{s}^{2}}{2 \pi^{4}}(\delta \mathbf{Y})^{2} \int_{\mathbf{w}, \mathbf{v}, \mathbf{x}, \mathbf{y}, \mathbf{z}, \mathbf{z}^{\prime}} \frac{X \cdot Y W^{\prime} \cdot V^{\prime}}{X^{2} Y^{2}\left(W^{\prime}\right)^{2}\left(V^{\prime}\right)^{2}} \\
& \times\left[S_{A}^{c d}\left(\mathbf{z}^{\prime}\right) J_{L}^{c}(\mathbf{w}) J_{L}^{a}(\mathbf{x}) J_{L}^{a}(\mathbf{y}) J_{R}^{d}(\mathbf{v})+S_{A}^{c d}\left(\mathbf{z}^{\prime}\right) J_{L}^{c}(\mathbf{v}) J_{R}^{d}(\mathbf{w}) J_{R}^{a}(\mathbf{x}) J_{R}^{a}(\mathbf{y})\right] \\
& -\frac{i f^{a b c} \alpha_{s}^{2}}{4 \pi^{4}}(\delta \mathbf{Y})^{2} \int_{\mathbf{w}, \mathbf{x}, \mathbf{y}, \mathbf{z}, \mathbf{z}^{\prime}}\left(\frac{X \cdot Y X^{\prime} \cdot W^{\prime}}{X^{2} Y^{2}\left(X^{\prime}\right)^{2}\left(W^{\prime}\right)^{2}}-\frac{X \cdot Y Y^{\prime} \cdot W^{\prime}}{X^{2} Y^{2}\left(Y^{\prime}\right)^{2}\left(W^{\prime}\right)^{2}}\right) \\
& \times\left[S_{A}^{c d}\left(\mathbf{z}^{\prime}\right) J_{L}^{b}(\mathbf{x}) J_{L}^{a}(\mathbf{y}) J_{R}^{d}(\mathbf{w})-S_{A}^{d c}\left(\mathbf{z}^{\prime}\right) J_{L}^{d}(\mathbf{w}) J_{R}^{a}(\mathbf{x}) J_{R}^{b}(\mathbf{y})\right]
\end{aligned}
$$

\subsection{Virtual contributions}

$\Sigma_{\text {virtual }}$ is defined in (4.9). This contribution comes from the scattering of the vacuum component $\mathcal{N}^{\mathrm{NLO}}|0\rangle$ of the LCWF. To save space, we will not illustrate this contribution, but, quite obviously, these are all the graphs with no soft partons crossing the shockwave, similarly to figure (3a, 3b). With (3.108) and (3.105) in mind, and counting powers of $g$,

$$
\begin{aligned}
\Sigma_{\text {virtual }}= & \left\langle 0\left|\left\|\mathcal{N}^{\mathrm{NLO}}\right\| \hat{S}\right| 0\right\rangle+\left\langle 0\left|\hat{S}\left\|\mathcal{N}^{\mathrm{NLO}}\right\|\right| 0\right\rangle+\frac{1}{4}\left\langle 0\left|\left\langle\psi_{g \rho}^{L O} \mid \psi_{g \rho}^{L O}\right\rangle \hat{S}\left\langle\psi_{g \rho}^{L O} \mid \psi_{g \rho}^{L O}\right\rangle\right| 0\right\rangle \\
& +\left\langle 0\left|\hat{S} i \phi^{\mathrm{NLO}}\right| 0\right\rangle-\left\langle 0\left|i \phi^{\mathrm{NLO} \dagger} \hat{S}\right| 0\right\rangle-2 .
\end{aligned}
$$

To organise the computation, we introduce the following decomposition for $\Sigma_{\text {virtual }}$,

$$
\Sigma_{\text {virtual }}=\Sigma_{v}^{L O}+\Sigma_{v J J}+\Sigma_{v J J J}+\Sigma_{v J J J}+\Sigma_{\phi} .
$$

where, after inserting $\left\|\mathcal{N}^{\mathrm{NLO}}\right\|$, as defined in equation (3.105), into (4.43),

$$
\Sigma_{v}^{L O} \equiv-\frac{1}{2}\left\langle 0\left|\left\langle\psi_{g \rho}^{L O} \mid \psi_{g \rho}^{L O}\right\rangle \hat{S}\right| 0\right\rangle-\frac{1}{2}\left\langle 0\left|\hat{S}\left\langle\psi_{g \rho}^{L O} \mid \psi_{g \rho}^{L O}\right\rangle\right| 0\right\rangle
$$

The contribution with two $J$ operators

$$
\begin{aligned}
\Sigma_{v J J} \equiv & \Sigma_{v q \bar{q}}+\Sigma_{v J \not J}+\Sigma_{v J \not \phi J J}, \\
\Sigma_{v q \bar{q}} \equiv & -\frac{1}{2}\left\langle 0\left|\left\langle\psi_{q \bar{q} \rho} \mid \psi_{q \bar{q} \rho}\right\rangle \hat{S}\right| 0\right\rangle-\frac{1}{2}\left\langle 0\left|\hat{S}\left\langle\psi_{q \bar{q} \rho} \mid \psi_{q \bar{q} \rho}\right\rangle\right| 0\right\rangle . \\
\Sigma_{v J \not J} \equiv & -\frac{1}{2}\left\langle 0\left|\left(\left\langle\psi_{g \rho}^{L O} \mid \psi_{g \rho}\right\rangle+\left\langle\psi_{g \rho} \mid \psi_{g \rho}^{L O}\right\rangle\right) \hat{S}\right| 0\right\rangle \\
& -\frac{1}{2}\left\langle 0\left|\hat{S}\left(\left\langle\psi_{g \rho}^{L O} \mid \psi_{g \rho}\right\rangle+\left\langle\psi_{g \rho} \mid \psi_{g \rho}^{L O}\right\rangle\right)\right| 0\right\rangle, \\
\Sigma_{v J \not \phi J} \equiv & -\frac{1}{2}\left\langle 0\left|\left\langle\psi_{g g \rho} \mid \psi_{g g \rho}\right\rangle \hat{S}\right| 0\right\rangle-\frac{1}{2}\left\langle 0\left|\hat{S}\left\langle\psi_{g g \rho} \mid \psi_{g g \rho}\right\rangle\right| 0\right\rangle .
\end{aligned}
$$

The contribution with three $J$ operators

$$
\begin{aligned}
\Sigma_{v J J J} \equiv & \Sigma_{v J J \not J}+\Sigma_{v J J \not \phi J} . \\
\Sigma_{v J J \phi \$ J} \equiv & -\frac{1}{2}\langle 0|\left(\left\langle\psi_{g g \rho \rho}\right| \psi_{g g} \rho+\left\langle\psi_{g g \rho} \mid \psi_{g g \rho \rho}\right\rangle\right) \hat{S}|0\rangle \\
& -\frac{1}{2}\left\langle 0\left|\hat{S}\left(\left\langle\psi_{g g \rho \rho} \mid \psi_{g g \rho}\right\rangle+\left\langle\psi_{g g \rho} \mid \psi_{g g \rho \rho}\right\rangle\right)\right| 0\right\rangle,
\end{aligned}
$$




$$
\begin{aligned}
\Sigma_{v J J \not J J} \equiv & -\frac{1}{2}\left\langle 0\left|\left(\left\langle\psi_{g \rho}^{L O} \mid \psi_{g \rho \rho}\right\rangle+\left\langle\psi_{g \rho \rho} \mid \psi_{g \rho}^{L O}\right\rangle\right) \hat{S}\right| 0\right\rangle \\
& -\frac{1}{2}\left\langle 0\left|\hat{S}\left(\left\langle\psi_{g \rho}^{L O} \mid \psi_{g \rho \rho}\right\rangle+\left\langle\psi_{g \rho \rho} \mid \psi_{g \rho}^{L O}\right\rangle\right)\right| 0\right\rangle .
\end{aligned}
$$

The contribution with four $J$ operators

$$
\begin{aligned}
\Sigma_{v J J J J} \equiv & \langle 0|\left(-\frac{1}{2}\left\langle\psi_{g \rho}^{L O} \mid \psi_{g \rho \rho \rho}\right\rangle-\frac{1}{2}\left\langle\psi_{g \rho \rho \rho} \mid \psi_{g \rho}^{L O}\right\rangle-\frac{1}{2}\left\langle\psi_{g g \rho \rho} \mid \psi_{g g \rho \rho}\right\rangle\right. \\
& \left.-\frac{1}{8}\left\langle\psi_{g \rho}^{L O} \mid \psi_{g \rho}^{L O}\right\rangle\left\langle\psi_{g \rho}^{L O} \mid \psi_{g \rho}^{L O}\right\rangle\right) \hat{S}|0\rangle+\langle 0| \hat{S}\left(-\frac{1}{2}\left\langle\psi_{g \rho}^{L O} \mid \psi_{g \rho \rho \rho}\right\rangle\right. \\
& \left.-\frac{1}{2}\left\langle\psi_{g \rho \rho \rho} \mid \psi_{g \rho}^{L O}\right\rangle-\frac{1}{2}\left\langle\psi_{g g \rho \rho} \mid \psi_{g g \rho \rho}\right\rangle-\frac{1}{8}\left\langle\psi_{g \rho}^{L O} \mid \psi_{g \rho}^{L O}\right\rangle\left\langle\psi_{g \rho}^{L O} \mid \psi_{g \rho}^{L O}\right\rangle\right)|0\rangle \\
& +\frac{1}{4}\left\langle 0\left|\left\langle\psi_{g \rho}^{L O} \mid \psi_{g \rho}^{L O}\right\rangle \hat{S}\left\langle\psi_{g \rho}^{L O} \mid \psi_{g \rho}^{L O}\right\rangle\right| 0\right\rangle .
\end{aligned}
$$

Finally,

$$
\Sigma_{\phi} \equiv\left\langle 0\left|\hat{S} i \phi^{\mathrm{NLO}}\right| 0\right\rangle-\left\langle 0\left|i \phi^{\mathrm{NLO} \dagger} \hat{S}\right| 0\right\rangle .
$$

In the rest of this section we present the results for all the virtual terms defined in (4.47)-(4.54). In fact, it is straightforward to deduce most of them by recycling our previous computations. We notice that most of the overlaps that appear in (4.47)-(4.53) could be read off from the corresponding real terms, when the limit $S \longrightarrow 1$ is taken. In addition, this limit has to be taken with understanding that all the factors $\rho$ in (4.47) - (4.53) (apart of the last line in (4.53)) are placed either on the left or right of $\hat{S}$. This basically implies a replacement in the real terms $J_{L} \longrightarrow J_{R}\left(J_{R} \longrightarrow J_{L}\right)$ in the case $\hat{S}$ is placed to the right (left) of the matrix elements. One should, however, be careful with the ordering of the operators. The correct procedure involves first writing all the $J_{R}$ operators to the left of $J_{L}$ operators. Second is to convert the $J$ operators back into $\rho$ in accord to (2.44). Finally, the string of $\rho$ gets converted again to a string of $J$ operators.

- Computation of $\boldsymbol{\Sigma}_{\boldsymbol{v} \boldsymbol{J} \boldsymbol{J}}$. The contribution (4.47) can be deduced from (4.15); (4.48) from (4.36) and (4.38); (4.49) from (4.19) and (4.20). (4.47) can be split similarly to how it was done for the real terms:

$$
\Sigma_{v J J}=\Sigma_{v J J}^{\mathrm{NLO}}+\Sigma_{v J J}^{(\delta \mathrm{Y})^{2}}
$$

with

$$
\Sigma_{v J J}^{(\delta Y)^{2}}=-\frac{\alpha_{s}^{2} N_{c}}{16 \pi^{4}}(\delta \mathbf{Y})^{2} \int_{\mathbf{x}, \mathbf{y}, \mathbf{z}, \mathbf{z}^{\prime}} \frac{X \cdot Y X^{\prime} \cdot Y^{\prime}}{X^{2} Y^{2}\left(X^{\prime}\right)^{2}\left(Y^{\prime}\right)^{2}}\left[J_{L}^{a}(\mathbf{x}) J_{L}^{a}(\mathbf{y})+J_{R}^{a}(\mathbf{x}) J_{R}^{a}(\mathbf{y})\right],
$$

and

$$
\Sigma_{v J J}^{\mathrm{NLO}}=-\delta \mathrm{Y} \int_{\mathbf{x}, \mathbf{y}, \mathbf{z}} K_{\mathrm{JSJ}}(\mathbf{x}, \mathbf{y}, \mathbf{z})\left[J_{L}^{a}(\mathbf{x}) J_{L}^{a}(\mathbf{y})+J_{R}^{a}(\mathbf{x}) J_{R}^{a}(\mathbf{y})\right],
$$

where we have introduced the following kernel:

$$
K_{\mathrm{JSJ}}(\mathbf{x}, \mathbf{y}, \mathbf{z}) \equiv K_{\mathrm{JSJ}}^{\prime}(\mathbf{x}, \mathbf{y}, \mathbf{z})-\frac{1}{2} \int_{\mathbf{z}^{\prime}} K_{q \bar{q}}\left(\mathbf{x}, \mathbf{y}, \mathbf{z}, \mathbf{z}^{\prime}\right)-\frac{N_{c}}{2} \int_{\mathbf{z}^{\prime}} K_{\mathrm{JSSJ}}\left(\mathbf{x}, \mathbf{y}, \mathbf{z}, \mathbf{z}^{\prime}\right) .
$$

The $z^{\prime}$ integrals are divergent and have to be properly regularised. The results are quoted in (D.5) and (D.6). The kernel $K_{\text {JSJ }}$ in (4.58) is the one defined in (2.66). 
- Computation of $\boldsymbol{\Sigma}_{\boldsymbol{v} \boldsymbol{J} \boldsymbol{J} \boldsymbol{J}}$. Taking the $S \rightarrow 1$ limit in (4.24), (4.25), (4.29), (4.30), and splitting (4.51) as usual,

$$
\Sigma_{v J J J}=\Sigma_{v J J J}^{\mathrm{NLO}}+\Sigma_{v J J}^{(\delta \mathrm{Y})^{2}}
$$

we obtain:

$$
\begin{aligned}
& \sum_{v J J J}^{\mathrm{NLO}}=-\frac{1}{2} \delta \mathrm{Y} \int_{\mathbf{w}, \mathbf{x}, \mathbf{y}, \mathbf{z}, \mathbf{z}^{\prime}}\left(K_{\mathrm{JJSSJ}}\left(\mathbf{w}, \mathbf{x}, \mathbf{y}, \mathbf{z}, \mathbf{z}^{\prime}\right)+K_{\mathrm{JJSJ}}(\mathbf{w}, \mathbf{x}, \mathbf{y}, \mathbf{z})\right) f^{a c b} \\
& \quad \times\left[J_{L}^{c}(\mathbf{x}) J_{L}^{b}(\mathbf{y}) J_{L}^{a}(\mathbf{w})-J_{L}^{a}(\mathbf{w}) J_{L}^{c}(\mathbf{x}) J_{L}^{b}(\mathbf{y})+J_{R}^{c}(\mathbf{x}) J_{R}^{b}(\mathbf{y}) J_{R}^{a}(\mathbf{w})-J_{R}^{a}(\mathbf{w}) J_{R}^{c}(\mathbf{x}) J_{R}^{b}(\mathbf{y})\right] .
\end{aligned}
$$

By using the identity (D.4) along with

$$
\begin{aligned}
& f^{a c b}\left[J_{L}^{c}(\mathbf{x}) J_{L}^{b}(\mathbf{y}) J_{L}^{a}(\mathbf{w})-J_{L}^{a}(\mathbf{w}) J_{L}^{c}(\mathbf{x}) J_{L}^{b}(\mathbf{y})+J_{R}^{c}(\mathbf{x}) J_{R}^{b}(\mathbf{y}) J_{R}^{a}(\mathbf{w})\right. \\
& \left.-J_{R}^{a}(\mathbf{w}) J_{R}^{c}(\mathbf{x}) J_{R}^{b}(\mathbf{y})\right]=i N_{c}\left[J_{L}^{a}(\mathbf{x}) J_{L}^{a}(\mathbf{y})-J_{R}^{a}(\mathbf{x}) J_{R}^{a}(\mathbf{y})\right][\delta(\mathbf{x}-\mathbf{w})-\delta(\mathbf{y}-\mathbf{w})],
\end{aligned}
$$

It is possible to show that:

$$
\Sigma_{v J J J}^{\mathrm{NLO}}=0
$$

and

$$
\Sigma_{v J J J}^{(\delta Y)^{2}}=-\frac{N_{c} \alpha_{s}^{2}}{2 \pi^{4}}(\delta \mathbf{Y})^{2} \int_{\mathbf{x}, \mathbf{y}, \mathbf{z}, \mathbf{z}^{\prime}} \frac{\left(X^{\prime}-Y^{\prime}\right)^{2} X \cdot Y}{2 X^{2} Y^{2}\left(Y^{\prime}\right)^{2}\left(X^{\prime}\right)^{2}}\left[J_{L}^{a}(\mathbf{x}) J_{L}^{a}(\mathbf{y})-J_{R}^{a}(\mathbf{x}) J_{R}^{a}(\mathbf{y})\right] .
$$

- Computation of $\boldsymbol{\Sigma}_{\text {JJJJ }}$. Taking the $S \rightarrow 1$ limit in (4.40) and (4.42), we arrive at the following expression for (4.53):

$$
\begin{aligned}
& \Sigma_{v J J J J}^{(\delta \mathbf{Y})^{2}}=\frac{\alpha_{s}^{2}}{8 \pi^{4}}(\delta \mathbf{Y})^{2} \int_{\mathbf{w}, \mathbf{v}, \mathbf{x}, \mathbf{y}, \mathbf{z}, \mathbf{z}^{\prime}} \frac{X \cdot Y W^{\prime} \cdot V^{\prime}}{X^{2} Y^{2}\left(W^{\prime}\right)^{2}\left(V^{\prime}\right)^{2}} \\
& \quad \times\left[J_{R}^{c}(\mathbf{w}) J_{R}^{c}(\mathbf{v}) J_{R}^{a}(\mathbf{x}) J_{R}^{a}(\mathbf{y})+J_{L}^{c}(\mathbf{w}) J_{L}^{c}(\mathbf{v}) J_{L}^{a}(\mathbf{x}) J_{L}^{a}(\mathbf{y})+2 J_{L}^{a}(\mathbf{x}) J_{L}^{a}(\mathbf{y}) J_{R}^{c}(\mathbf{w}) J_{R}^{c}(\mathbf{v})\right] \\
& \quad+\frac{\alpha_{s}^{2} N_{c}}{16 \pi^{4}}(\delta \mathbf{Y})^{2} \int_{\mathbf{x}, \mathbf{y}, \mathbf{z}, \mathbf{z}^{\prime}} \frac{X \cdot Y X^{\prime} \cdot Y^{\prime}}{X^{2} Y^{2}\left(X^{\prime}\right)^{2}\left(Y^{\prime}\right)^{2}}\left[J_{R}^{a}(\mathbf{x}) J_{R}^{a}(\mathbf{y})+J_{L}^{a}(\mathbf{x}) J_{L}^{a}(\mathbf{y})\right] \\
& \quad+\frac{N_{c} \alpha_{s}^{2}}{2 \pi^{4}}(\delta \mathbf{Y})^{2} \int_{\mathbf{x}, \mathbf{y}, \mathbf{z}, \mathbf{z}^{\prime}} \frac{\left(X^{\prime}-Y^{\prime}\right)^{2} X \cdot Y}{2 X^{2} Y^{2}\left(Y^{\prime}\right)^{2}\left(X^{\prime}\right)^{2}}\left[J_{L}^{a}(\mathbf{x}) J_{L}^{a}(\mathbf{y})-J_{R}^{a}(\mathbf{x}) J_{R}^{a}(\mathbf{y})\right]
\end{aligned}
$$

- Computation of $\boldsymbol{\Sigma}_{\boldsymbol{\phi}} . \quad \Sigma_{\phi}$ is defined in (4.54) and computed in appendix F from the condition (3.31),

$$
\begin{aligned}
\Sigma_{\phi}^{\mathrm{NLO}}= & -\delta \mathrm{Y} \int_{\mathbf{w}, \mathbf{x}, \mathbf{y}, \mathbf{z}, \mathbf{z}^{\prime}} K_{\mathrm{JJSSJ}}\left(\mathbf{w}, \mathbf{x}, \mathbf{y}, \mathbf{z}, \mathbf{z}^{\prime}\right) \\
& \times f^{a c b}\left[J_{L}^{a}(\mathbf{x}) J_{L}^{b}(\mathbf{y}) J_{L}^{c}(\mathbf{w})-J_{R}^{a}(\mathbf{x}) J_{R}^{b}(\mathbf{y}) J_{R}^{c}(\mathbf{w})\right] .
\end{aligned}
$$

With the help of identities (D.4) and (D.1) it is possible to cast $\Sigma_{\phi}$ into the form that is straightforwardly comparable with (2.56),

$$
\begin{aligned}
\Sigma_{\phi}^{\mathrm{NLO}}= & -\frac{1}{3} \delta \mathrm{Y} \int_{\mathbf{w}, \mathbf{x}, \mathbf{y}, \mathbf{z}, \mathbf{z}^{\prime}}\left(K_{\mathrm{JJSSJ}}\left(\mathbf{w}, \mathbf{x}, \mathbf{y}, \mathbf{z}, \mathbf{z}^{\prime}\right)+K_{\mathrm{JJSJ}}(\mathbf{w}, \mathbf{x}, \mathbf{y}, \mathbf{z})\right) f^{a c b} \\
& \times\left[J_{L}^{c}(\mathbf{x}) J_{L}^{b}(\mathbf{y}) J_{L}^{a}(\mathbf{w})-J_{R}^{c}(\mathbf{x}) J_{R}^{b}(\mathbf{y}) J_{R}^{a}(\mathbf{w})\right]
\end{aligned}
$$


- Assembling the virtual contributions. $\Sigma_{\text {virtual }}$ has contributions proportional to $\delta \mathrm{Y}$ and $(\delta \mathrm{Y})^{2}$ and we split them via our standard procedure:

$$
\Sigma_{\text {virtual }}=\Sigma_{\text {virtual }}^{\mathrm{NLO}}+\Sigma_{\text {virtual }}^{(\delta)^{2}}
$$

We define $\Sigma_{\text {virtual }}^{\mathrm{NLO}}$ by adding together contributions (4.56), (4.64) and (4.66):

$$
\begin{aligned}
& \Sigma_{\text {virtual }}^{\mathrm{NLO}} \equiv \Sigma_{v J J J}^{\mathrm{NLO}}+\Sigma_{v J J}^{\mathrm{NLO}}+\Sigma_{\phi}^{\mathrm{NLO}}=-\delta \mathrm{Y} \int_{\mathbf{x}, \mathbf{y}, \mathbf{z}} K_{\mathrm{JSJ}}(\mathbf{x}, \mathbf{y}, \mathbf{z})\left[J_{L}^{a}(\mathbf{x}) J_{L}^{a}(\mathbf{y})\right. \\
& \left.\quad+J_{R}^{a}(\mathbf{x}) J_{R}^{a}(\mathbf{y})\right]-\frac{1}{3} \delta \mathrm{Y} \int_{\mathbf{w}, \mathbf{x}, \mathbf{y}, \mathbf{z}, \mathbf{z}^{\prime}}\left(K_{\mathrm{JJSSJ}}\left(\mathbf{w}, \mathbf{x}, \mathbf{y}, \mathbf{z}, \mathbf{z}^{\prime}\right)+K_{\mathrm{JJSJ}}(\mathbf{w}, \mathbf{x}, \mathbf{y}, \mathbf{z})\right) f^{a c b} \\
& \quad \times\left[J_{L}^{c}(\mathbf{x}) J_{L}^{b}(\mathbf{y}) J_{L}^{a}(\mathbf{w})-J_{R}^{c}(\mathbf{x}) J_{R}^{b}(\mathbf{y}) J_{R}^{a}(\mathbf{w})\right]
\end{aligned}
$$

We define $\Sigma_{\text {virtual }}^{(\delta Y)^{2}}$ by adding together the contributions (4.56), (4.63), and (4.64):

$$
\begin{aligned}
\Sigma_{\text {virtual }}^{(\delta Y)^{2}} \equiv & \Sigma_{v J J J}^{(\delta Y)^{2}}+\Sigma_{v J J J}^{(\delta Y)^{2}}+\Sigma_{v J J}^{(\delta Y)^{2}}=\frac{\alpha_{s}^{2}}{8 \pi^{4}}(\delta \mathbf{Y})^{2} \int_{\mathbf{w}, \mathbf{v}, \mathbf{x}, \mathbf{y}, \mathbf{z}, \mathbf{z}^{\prime}} \frac{X \cdot Y W^{\prime} \cdot V^{\prime}}{X^{2} Y^{2}\left(W^{\prime}\right)^{2}\left(V^{\prime}\right)^{2}} \\
& \times\left[J_{L}^{c}(\mathbf{w}) J_{L}^{c}(\mathbf{v})+J_{R}^{c}(\mathbf{w}) J_{R}^{c}(\mathbf{v})\right]\left[J_{L}^{a}(\mathbf{x}) J_{L}^{a}(\mathbf{y})+J_{R}^{a}(\mathbf{x}) J_{R}^{a}(\mathbf{y})\right] .
\end{aligned}
$$

\subsection{The NLO JIMWLK Hamiltonian assembled}

We define $\Sigma^{\mathrm{NLO}}$ as a sum of all the contributions of order $\delta \mathrm{Y}$ :

$$
\Sigma^{\mathrm{NLO}} \equiv \Sigma_{\mathrm{JSJ}}^{\mathrm{NLO}}+\Sigma_{q \bar{q}}^{\mathrm{NLO}}+\Sigma_{\text {JSSJ }}^{\mathrm{NLO}}+\Sigma_{\text {JJSJ }}^{\mathrm{NLO}}+\Sigma_{\text {JJSSJ }}^{\mathrm{NLO}}+\Sigma_{\text {virtual }}^{\mathrm{NLO}},
$$

with the terms computed in (4.15), (4.24), (4.19), (4.29), (4.35), (4.68). The integral over $z$ in $\Sigma_{\text {JSJ }}^{\mathrm{NLO}}$ has UV divergence. Similar divergence is found in $\Sigma_{\text {JSSJ }}^{\mathrm{NLO}}(2.59)$ and $\Sigma_{q \bar{q}}^{\mathrm{NLO}}(2.67)$ when $\mathbf{z} \rightarrow \mathbf{z}^{\prime}$. Following [47], we subtract (and later add) terms which correspond to the case in which both gluons or the quark and anti-quark pair pass through the shockwave at the same transverse point. This corresponds to single gluon scattering, thanks to the identities $S_{A}^{a b}(\mathbf{z})=2 \operatorname{tr}\left[S^{\dagger}(\mathbf{z}) t^{a} S(\mathbf{z}) t^{b}\right]$ and $f^{a b c} f^{\text {def }} S_{A}^{b e}(\mathbf{z}) S_{A}^{c f}(\mathbf{z})=N_{c} S_{A}^{a d}(\mathbf{z})$.

Accordingly, we introduce the subtraction terms

$$
\begin{aligned}
\Sigma_{q \bar{q}}^{\mathrm{sub}} & \equiv-\delta \mathrm{Y} \int_{\mathbf{x}, \mathbf{y}, \mathbf{z}, \mathbf{z}^{\prime}} K_{q \bar{q}}\left(\mathbf{x}, \mathbf{y}, \mathbf{z}, \mathbf{z}^{\prime}\right) J_{L}^{a}(\mathbf{x}) S_{A}^{a b}(\mathbf{z}) J_{R}^{b}(\mathbf{y}) . \\
\Sigma_{\mathrm{JSSJ}}^{\mathrm{sub}} & \equiv-\delta \mathrm{Y} \int_{\mathbf{x}, \mathbf{y}, \mathbf{z}, \mathbf{z}^{\prime}} K_{\mathrm{JSSJ}}\left(\mathbf{x}, \mathbf{y}, \mathbf{z}, \mathbf{z}^{\prime}\right) N_{c} J_{L}^{a}(\mathbf{x}) S_{A}^{a b}(\mathbf{z}) J_{R}^{b}(\mathbf{y}) .
\end{aligned}
$$

As was mentioned in the previous subsection, proper dimensional regularisation of $z^{\prime}$ integrals is needed, and the results for the integrations are quoted in (D.5) and (D.6). With the subtraction terms added,

$$
\begin{aligned}
\Sigma^{\mathrm{NLO}}= & \left(\Sigma_{\mathrm{JSJ}}^{\mathrm{NLO}}+\Sigma_{q \bar{q}}^{\mathrm{sub}}+\Sigma_{\mathrm{JSSJ}}^{\mathrm{sub}}\right)+\left(\Sigma_{\mathrm{JSSJ}}^{\mathrm{NLO}}-\Sigma_{\text {JSSJ }}^{\mathrm{sub}}\right)+\left(\Sigma_{q \bar{q}}^{\mathrm{NLO}}-\Sigma_{q \bar{q}}^{\mathrm{sub}}\right) \\
& +\Sigma_{\text {JJSJ }}^{\mathrm{NLO}}+\Sigma_{\text {JJSSJ }}^{\mathrm{NLO}}+\Sigma_{\text {virtual }}^{\mathrm{NLO}},
\end{aligned}
$$

Following (4.58),

$$
\Sigma_{\mathrm{JSJ}}^{\mathrm{NLO}}+\Sigma_{q \bar{q}}^{\mathrm{sub}}+\Sigma_{\mathrm{JSSJ}}^{\mathrm{sub}}=-\delta \mathrm{Y} \int_{\mathbf{x}, \mathbf{y}, \mathbf{z}} K_{\mathrm{JSJ}}(\mathbf{x}, \mathbf{y}, \mathbf{z})\left[-2 J_{L}^{a}(\mathbf{x}) S_{A}^{a b}(\mathbf{z}) J_{R}^{b}(\mathbf{y})\right],
$$

The UV divergence is canceled in $K_{\text {JSJ }}$. The NLO JIMWLK Hamiltonian is obtained from (4.73) via the definition (1.6). The result is identical to the one quoted in (2.56). 


\subsection{Reduction of the $(\mathrm{LO})^{2}$ contribution}

In addition to the contributions to the NLO JIMWLK Hamiltonian we found terms proportional to $(\delta \mathrm{Y})^{2}$ :

$$
\Sigma^{(\delta Y)^{2}} \equiv \Sigma_{\text {JSJ }}^{(\delta Y)^{2}}+\Sigma_{\text {JJSJ }}^{(\delta Y)^{2}}+\Sigma_{\text {JJJSJ }}^{(\delta Y)^{2}}+\Sigma_{\text {JSSJ }}^{(\delta Y}+\Sigma_{\text {JJSSJ }}^{(\delta Y)^{2}}+\Sigma_{\text {JJSSJJ }}^{(\delta Y)^{2}}+\Sigma_{\text {virtual }}^{(\delta Y)^{2}} .
$$

Our objective in this section is to show that all these contributions are generated by second iteration of the LO Hamiltonian $H_{\text {JIMWLK }}^{L O}$ Let's first summarise all the $(\delta \mathrm{Y})^{2}$ contributions that were found in subsections 4.3-4.7. From (4.69),

$$
\begin{aligned}
\Sigma_{\text {virtual }}^{(\delta \mathbf{Y})^{2}} & \equiv \frac{\alpha_{s}^{2}}{8 \pi^{4}}(\delta \mathbf{Y})^{2} \int_{\mathbf{w}, \mathbf{v}, \mathbf{x}, \mathbf{y}, \mathbf{z}, \mathbf{z}^{\prime}} \frac{X \cdot Y W^{\prime} \cdot V^{\prime}}{X^{2} Y^{2}\left(W^{\prime}\right)^{2}\left(V^{\prime}\right)^{2}} \\
& \times\left[J_{L}^{b}(\mathbf{w}) J_{L}^{b}(\mathbf{v})+J_{R}^{b}(\mathbf{w}) J_{R}^{b}(\mathbf{v})\right]\left[J_{L}^{a}(\mathbf{x}) J_{L}^{a}(\mathbf{y})+J_{R}^{a}(\mathbf{x}) J_{R}^{a}(\mathbf{y})\right] .
\end{aligned}
$$

Adding together the contribution with one $S$ factor, (4.30), (4.38) and (4.42):

$$
\begin{aligned}
& \Sigma_{\mathrm{JSJ}}^{(\delta \mathrm{Y})^{2}}+\Sigma_{\mathrm{JJSJ}}^{(\delta \mathrm{Y})^{2}}+\Sigma_{\mathrm{JJJSJ}}^{(\delta \mathrm{Y})^{2}}=-\frac{\alpha_{s}^{2}}{2 \pi^{4}}(\delta \mathbf{Y})^{2}\left(\int_{\mathbf{w}, \mathbf{v}, \mathbf{x}, \mathbf{y}, \mathbf{z}, \mathbf{z}^{\prime}} \frac{X \cdot Y W^{\prime} \cdot V^{\prime}}{X^{2} Y^{2}\left(W^{\prime}\right)^{2}\left(V^{\prime}\right)^{2}}\right. \\
& \times\left[S_{A}^{c d}\left(\mathbf{z}^{\prime}\right) J_{L}^{c}(\mathbf{w}) J_{L}^{a}(\mathbf{x}) J_{L}^{a}(\mathbf{y}) J_{R}^{d}(\mathbf{v})+S_{A}^{c d}\left(\mathbf{z}^{\prime}\right) J_{L}^{c}(\mathbf{v}) J_{R}^{d}(\mathbf{w}) J_{R}^{a}(\mathbf{x}) J_{R}^{a}(\mathbf{y})\right] \\
& +\frac{i f^{a b c}}{2} \int_{\mathbf{w}, \mathbf{x}, \mathbf{y}, \mathbf{z}, \mathbf{z}^{\prime}}\left(\frac{X \cdot Z Y^{\prime} \cdot W^{\prime}}{X^{2} Z^{2}\left(Y^{\prime}\right)^{2}\left(W^{\prime}\right)^{2}}-\frac{Y \cdot Z X^{\prime} \cdot W^{\prime}}{Y^{2} Z^{2}\left(X^{\prime}\right)^{2}\left(W^{\prime}\right)^{2}}\right) \\
& \times\left[S_{A}^{c d}\left(\mathbf{z}^{\prime}\right) J_{L}^{a}(\mathbf{x}) J_{L}^{b}(\mathbf{y}) J_{R}^{d}(\mathbf{w})-S_{A}^{d c}\left(\mathbf{z}^{\prime}\right) J_{L}^{d}(\mathbf{w}) J_{R}^{a}(\mathbf{x}) J_{R}^{b}(\mathbf{y})\right] \\
& \left.+\frac{N_{c}}{2} \int_{\mathbf{x}, \mathbf{y}, \mathbf{z}, \mathbf{z}^{\prime}}\left(\frac{2 X \cdot Y}{Z^{2} X^{2} Y^{2}}-\frac{X \cdot Y Z \cdot Y^{\prime}}{X^{2} Y^{2} Z^{2}\left(Y^{\prime}\right)^{2}}-\frac{X \cdot Y X^{\prime} \cdot Z}{X^{2} Y^{2}\left(X^{\prime}\right)^{2} Z^{2}}\right) S_{A}^{a b}(\mathbf{z}) J_{L}^{a}(\mathbf{x}) J_{R}^{b}(\mathbf{y})\right) .
\end{aligned}
$$

Adding together the contributions which contain two $S$ factors, (4.25), (4.20), and (4.40):

$$
\begin{aligned}
& \Sigma_{\text {JSSJ }}^{(\delta \mathbf{Y})^{2}}+\Sigma_{\text {JJSSJ }}^{(\delta \mathbf{Y})^{2}}+\Sigma_{\text {JJSSJJ }}^{(\delta \mathbf{Y})^{2}}=\frac{\alpha_{s}^{2}}{2 \pi^{4}}(\delta \mathbf{Y})^{2}\left(\int_{\mathbf{w}, \mathbf{v}, \mathbf{x}, \mathbf{y}, \mathbf{z}, \mathbf{z}^{\prime}} \frac{X \cdot Y W^{\prime} \cdot V^{\prime}}{X^{2} Y^{2}\left(W^{\prime}\right)^{2}\left(V^{\prime}\right)^{2}}\right. \\
& \quad \times S_{A}^{c d}\left(\mathbf{z}^{\prime}\right) S_{A}^{a b}(\mathbf{z}) J_{L}^{a}(\mathbf{x}) J_{L}^{c}(\mathbf{w}) J_{R}^{b}(\mathbf{y}) J_{R}^{d}(\mathbf{v})+\frac{i f^{a b c}}{2} \int_{\mathbf{w}, \mathbf{x}, \mathbf{y}, \mathbf{z}, \mathbf{z}^{\prime}}\left(\frac{X \cdot Z Y^{\prime} \cdot W^{\prime}}{X^{2} Z^{2}\left(Y^{\prime}\right)^{2}\left(W^{\prime}\right)^{2}}\right. \\
& \left.\quad-\frac{Y \cdot Z X^{\prime} \cdot W^{\prime}}{Y^{2} Z^{2}\left(X^{\prime}\right)^{2}\left(W^{\prime}\right)^{2}}\right)\left[S_{A}^{d b}(\mathbf{z}) S_{A}^{e a}\left(\mathbf{z}^{\prime}\right) J_{L}^{d}(\mathbf{x}) J_{L}^{e}(\mathbf{y}) J_{R}^{c}(\mathbf{w})\right. \\
& \left.-S_{A}^{a e}\left(\mathbf{z}^{\prime}\right) S_{A}^{b d}(\mathbf{z}) J_{L}^{c}(\mathbf{w}) J_{R}^{d}(\mathbf{x}) J_{R}^{e}(\mathbf{y})\right]+\frac{1}{2} \int_{\mathbf{x}, \mathbf{y}, \mathbf{z}, \mathbf{z}^{\prime}}\left(\frac{2 X \cdot Y}{Z^{2} X^{2} Y^{2}}\right. \\
& \left.\left.-\frac{X \cdot Y Z \cdot Y^{\prime}}{X^{2} Y^{2} Z^{2}\left(Y^{\prime}\right)^{2}}-\frac{X \cdot Y X^{\prime} \cdot Z}{X^{2} Y^{2}\left(X^{\prime}\right)^{2} Z^{2}}\right) f^{a b c} f^{d e f} S_{A}^{b e}(\mathbf{z}) S_{A}^{c f}\left(\mathbf{z}^{\prime}\right) J_{L}^{a}(\mathbf{x}) J_{R}^{d}(\mathbf{y})\right)
\end{aligned}
$$

Now let us write down the result that is obtained by applying $H^{L O}$ twice:

$$
\begin{aligned}
& (\delta \mathrm{Y})^{2}\left(H_{\mathrm{JIMWLK}}^{L O}\right)^{2}=\left(\Sigma^{L O}\right)^{2}=\frac{\alpha_{s}^{2}}{4 \pi^{4}}(\delta \mathrm{Y})^{2} \int_{\mathbf{w}, \mathbf{v}, \mathbf{x}, \mathbf{y}, \mathbf{z}, \mathbf{z}^{\prime}} \frac{X \cdot Y W^{\prime} \cdot V^{\prime}}{X^{2} Y^{2}\left(W^{\prime}\right)^{2}\left(V^{\prime}\right)^{2}} \\
& \quad \times\left[J_{L}^{a}(\mathbf{x}) J_{L}^{a}(\mathbf{y})+J_{R}^{a}(\mathbf{x}) J_{R}^{a}(\mathbf{y})-2 J_{L}^{a}(\mathbf{x}) S_{A}^{a b}(\mathbf{z}) J_{R}^{b}(\mathbf{y})\right] \\
& \quad \times\left[J_{L}^{c}(\mathbf{w}) J_{L}^{c}(\mathbf{v})+J_{R}^{c}(\mathbf{w}) J_{R}^{c}(\mathbf{v})-2 J_{L}^{c}(\mathbf{w}) S_{A}^{c d}\left(\mathbf{z}^{\prime}\right) J_{R}^{d}(\mathbf{v})\right] .
\end{aligned}
$$


$\left(\Sigma^{L O}\right)^{2}$ can also be split in accord with the number of $S$ factors:

$$
\left(\Sigma^{L O}\right)^{2}=\Sigma_{\text {JJJJ }}^{(L O)^{2}}+\Sigma_{\text {JJJSJ }}^{(L O)^{2}}+\Sigma_{\text {JJSSJJ }}^{(L O)^{2}}
$$

with

$$
\begin{aligned}
\Sigma_{\mathrm{JJJJ}}^{(L O)^{2}} \equiv & \frac{\alpha_{s}^{2}}{4 \pi^{4}}(\delta \mathrm{Y})^{2} \int_{\mathbf{w}, \mathbf{v}, \mathbf{x}, \mathbf{y}, \mathbf{z}, \mathbf{z}^{\prime}} \frac{X \cdot Y W^{\prime} \cdot V^{\prime}}{X^{2} Y^{2}\left(W^{\prime}\right)^{2}\left(V^{\prime}\right)^{2}} \\
& \times\left[J_{L}^{a}(\mathbf{x}) J_{L}^{a}(\mathbf{y})+J_{R}^{a}(\mathbf{x}) J_{R}^{a}(\mathbf{y})\right]\left[J_{L}^{c}(\mathbf{w}) J_{L}^{c}(\mathbf{v})+J_{R}^{c}(\mathbf{w}) J_{R}^{c}(\mathbf{v})\right] \\
\Sigma_{\mathrm{JJJSJ}}^{(L O)^{2}} \equiv & -\frac{\alpha_{s}^{2}}{2 \pi^{4}}(\delta \mathbf{Y})^{2} \int_{\mathbf{w}, \mathbf{v}, \mathbf{x}, \mathbf{y}, \mathbf{z}, \mathbf{z}^{\prime}} \frac{X \cdot Y W^{\prime} \cdot V^{\prime}}{X^{2} Y^{2}\left(W^{\prime}\right)^{2}\left(V^{\prime}\right)^{2}}\left(\left[J_{L}^{a}(\mathbf{x}) J_{L}^{a}(\mathbf{y})+J_{R}^{a}(\mathbf{x}) J_{R}^{a}(\mathbf{y})\right]\right. \\
& \left.\times\left[S_{A}^{c d}\left(\mathbf{z}^{\prime}\right) J_{L}^{c}(\mathbf{w}) J_{R}^{d}(\mathbf{v})\right]+\left[S_{A}^{a b}(\mathbf{z}) J_{L}^{a}(\mathbf{x}) J_{R}^{b}(\mathbf{y})\right]\left[J_{L}^{c}(\mathbf{w}) J_{L}^{c}(\mathbf{v})+J_{R}^{c}(\mathbf{w}) J_{R}^{c}(\mathbf{v})\right]\right) \\
\Sigma_{\mathrm{JJSSJJ}}^{(L O)^{2}} \equiv & \frac{\alpha_{s}^{2}}{\pi^{4}}(\delta \mathrm{Y})^{2} \int_{\mathbf{w}, \mathbf{v}, \mathbf{x}, \mathbf{y}, \mathbf{z}, \mathbf{z}^{\prime}} \frac{X \cdot Y W^{\prime} \cdot V^{\prime}}{X^{2} Y^{2}\left(W^{\prime}\right)^{2}\left(V^{\prime}\right)^{2}} \\
& \times\left[S_{A}^{a b}(\mathbf{z}) J_{L}^{a}(\mathbf{x}) J_{R}^{b}(\mathbf{y})\right]\left[S_{A}^{c d}\left(\mathbf{z}^{\prime}\right) J_{L}^{c}(\mathbf{w}) J_{R}^{d}(\mathbf{v})\right]
\end{aligned}
$$

In order to compare with $\Sigma^{(\delta Y)^{2}}$, we have to drag all the Wilson lines $S$ to the left of all $J$. This is done using the algebra (2.45) and (2.46), which generate terms with lesser number of $J$ operators. The procedure is applied both to $\Sigma_{\text {JJJSJ }}^{(L O)^{2}}$ and $\Sigma_{\text {JJSSJJ }}^{(L O)^{2}}$. By comparing (4.76) with (4.81), (4.77) with (4.82), and (4.78) with (4.83), we arrive at the following equivalence relations:

$$
\begin{aligned}
\left.\Sigma_{\text {JJJSJ }}^{(\delta \mathrm{Y})^{2}}+\Sigma_{\text {JJSJ }}^{(\delta \mathrm{Y})^{2}}+\Sigma_{\text {JSJ }}^{(\delta Y}\right)^{2} & =\frac{1}{2} \Sigma_{\text {JJJSJ }}^{(L O)^{2}} ; \\
\Sigma_{\text {JJSSJJ }}^{(\delta Y)^{2}}+\Sigma_{\text {JJSSJ }}^{(\delta Y)^{2}}+\Sigma_{\text {JSSJ }}^{(\delta Y)^{2}} & =\frac{1}{2} \Sigma_{\text {JJSSJJ }}^{(L O)^{2}} \\
\Sigma_{\text {virtual }}^{(\delta Y)^{2}} & =\frac{1}{2} \Sigma_{\text {JJJJ }}^{(L O)^{2}}
\end{aligned}
$$

This establishes (4.11).

\section{Acknowledgments}

We are most grateful to Ian Balitsky, Guillaume Beuf, and Alex Kovner, who have contributed to our work a great deal of inspiration and wisdom, and without whose support our progress would be noticeably slower. We also benefited from discussions with Nestor Armesto, Simon Caron-Huot, Andrey Grabovsky, and Yura Kovchegov. Y.M. thanks the Physics Department of the University of Connecticut for their hospitality during the times when part of this work was done. This work was supported by the ISRAELI SCIENCE FOUNDATION grant \#87277111, BSF grant \#012124, the People Program (Marie Curie Actions) of the European Union's Seventh Framework under REA grant agreement \#318921. 


\section{A From QCD Lagrangian to light cone QCD Hamiltonian}

The QCD Lagrangian:

$$
\mathcal{L}_{\mathrm{QCD}}=-\frac{1}{4} F^{a \mu \nu} F_{\mu \nu}^{a}+\sum_{f} \bar{\psi}_{f}(x)\left(i \gamma^{\mu} D_{\mu}-m_{f}\right) \psi_{f}(x) .
$$

The tensor $F_{\mu \nu}$ defined as $F_{\mu \nu} \equiv t^{a} F_{\mu \nu}^{a}=-\frac{i}{g}\left[D_{\mu}, D_{v}\right]$, satisfies the following equations of motion:

$$
D_{\mu} F^{a \mu \nu}=\partial_{\mu} F^{a \mu \nu}-g f^{a b c} A_{\mu}^{b} F^{c \mu \nu}=g \bar{\psi} \gamma^{\nu} t^{a} \psi \equiv-g J^{a \nu} .
$$

The canonical momenta:

$$
\left(\Pi_{A}\right)^{a \mu}(x) \equiv \frac{\delta \mathcal{L}_{\mathrm{QCD}}}{\delta\left(\partial^{-} A_{\mu}^{a}\right)}=\frac{1}{2} F^{a \mu+}, \quad\left(\Pi_{\psi}\right)(x) \equiv \frac{\delta \mathcal{L}_{\mathrm{QCD}}}{\delta\left(\partial^{-} \psi\right)}=\frac{i}{2} \bar{\psi} \gamma^{+} .
$$

Neglecting the quark masses, the Dirac equation becomes (after multiplication by $\gamma^{0}$ ):

$$
\left(i \gamma^{0} \gamma^{+} D_{+}+i \gamma^{0} \gamma^{-} D_{-}+i \alpha^{i} D_{i}\right) \psi=0 .
$$

with $\alpha^{i} \equiv \gamma^{0} \gamma^{i}$. Introduce projecting operators $\Lambda_{ \pm}=\Lambda^{ \pm}=\frac{1}{2} \gamma^{0} \gamma_{ \pm}$, where $\left(\Lambda_{ \pm}\right)^{2}=\Lambda_{ \pm}$ and $\Lambda_{+} \Lambda_{-}=\Lambda_{-} \Lambda_{+}=0$, such that $\widetilde{\psi}_{ \pm} \equiv \Lambda_{ \pm} \psi$ and also $\psi=\widetilde{\psi}_{+}+\widetilde{\psi}_{-}$,

$$
\psi=\left(\begin{array}{c}
\psi_{1} \\
\psi_{2} \\
\psi_{3} \\
\psi_{4}
\end{array}\right), \quad \widetilde{\psi}_{+}=\left(\begin{array}{c}
0 \\
\psi_{2} \\
\psi_{3} \\
0
\end{array}\right), \quad \widetilde{\psi}_{-}=\left(\begin{array}{c}
\psi_{1} \\
0 \\
0 \\
\psi_{4} \cdot
\end{array}\right)
$$

Two-coulmn vector $\psi_{+}=\left(\begin{array}{c}\psi_{2} \\ \psi_{3}\end{array}\right)$ and $\psi_{-}=\left(\begin{array}{c}\psi_{1} \\ \psi_{4}\end{array}\right)$ are defined by keeping only the nonvanishing components. The equations of motion for $\psi_{ \pm}$can be found after multiplying equation (A.4) by the projections $\Lambda_{ \pm}$:

$$
\left(i \partial^{-}-g t^{a} A^{a-}\right) \psi_{+}=-i \sigma^{i} D_{i} \psi_{-}, \quad\left(i \partial^{+}-g t^{a} A^{a+}\right) \psi_{-}=-i \sigma^{i} D_{i} \psi_{+},
$$

from which $\psi_{-}$is determined in terms of $\psi_{+}$. The following constraints are obtained from the + component of (A.2):

$$
D_{\mu} F^{a \mu+}=-g J^{a+}, \quad \partial^{+} A_{+}^{a}=-\frac{1}{\partial^{+}}\left(D_{i}^{a b} \partial^{+} A_{i}^{b}-g J^{a+}\right) .
$$

Eliminating $\psi_{-}$and $A_{+}$, the LC Hamiltonian is:

$$
\begin{aligned}
H_{L C Q C D} & =P_{+}=\int d x^{-} d^{2} \mathbf{x} \mathcal{H}_{\mathrm{QCD}}=\int d x^{-} d^{2} \mathbf{x}\left(\left(\Pi_{\psi}\right) \partial^{-} \psi+\left(\Pi_{A}\right)^{\mu} \partial^{-} A_{\mu}-\mathcal{L}_{\mathrm{QCD}}\right) \\
& =\int d x^{-} d^{2} \mathbf{x}\left(\frac{i}{2} \bar{\psi} \gamma^{+} \partial^{-} \psi-F^{\mu+} F_{\mu+}+\frac{1}{4} F^{\mu \nu} F_{\mu \nu}-i \bar{\psi} \gamma^{\mu} D_{\mu} \psi\right) .
\end{aligned}
$$

(2.4) and (2.5) are obtained using the following relations:

$$
\begin{aligned}
& F^{i j} F_{i j}=2\left(\left(\partial_{i} A_{j}^{a}\right)\left(\partial_{i} A_{j}^{a}\right)-\left(\partial_{i} A_{j}^{a}\right)\left(\partial_{j} A_{i}^{a}\right)\right)-4 g f^{a b c} A_{i}^{b} A_{j}^{c} \partial_{i} A_{j}^{a}+g^{2} f^{a b c} f^{a d e} A_{i}^{b} A_{j}^{c} A_{i}^{d} A_{j}^{e} . \\
& \Pi^{a}\left(x^{-}, \mathbf{x}\right) \Pi^{a}\left(x^{-}, \mathbf{x}\right) \\
& =\left(\frac{1}{\partial^{+}} D_{i} \partial^{+} A_{i}\right)^{a}\left(\frac{1}{\partial^{+}} D_{j} \partial^{+} A_{j}\right)^{a}+2 g\left(\frac{1}{\partial^{+}} D_{i} \partial^{+} A_{i}\right)^{a} \frac{1}{\partial^{+}} J^{a+}+g^{2} \frac{1}{\partial^{+}} J^{a+} \frac{1}{\partial^{+}} J^{a+} .
\end{aligned}
$$




\section{B Deriving the eikonal approximation for LC Hamiltonian}

In order to introduce the eikonal approximation for the LC QCD Hamiltonian, we split the gluon and quark fields into the soft and valence modes:

$$
\underline{A}_{i}^{a}(x)=\int_{\Lambda}^{e^{\delta \curlyvee} \Lambda} \frac{d k^{+}}{2 \pi} \int \frac{d^{2} \mathbf{k}}{(2 \pi)^{2}} \frac{1}{\sqrt{2 k^{+}}}\left(a_{i}^{a}\left(k^{+}, \mathbf{k}\right) e^{-i k \cdot x}+a_{i}^{a \dagger}\left(k^{+}, \mathbf{k}\right) e^{i k \cdot x}\right),
$$

and

$$
\bar{A}_{i}^{a}(x)=\int_{e^{\delta \curlyvee} \Lambda}^{\infty} \frac{d k^{+}}{2 \pi} \int \frac{d^{2} \mathbf{k}}{(2 \pi)^{2}} \frac{1}{\sqrt{2 k^{+}}}\left(a_{i}^{a}\left(k^{+}, \mathbf{k}\right) e^{-i k \cdot x}+a_{i}^{a \dagger}\left(k^{+}, \mathbf{k}\right) e^{i k \cdot x}\right) .
$$

Similarly for the quark field:

$$
\underline{\psi}_{+}^{\alpha}(x)=\sum_{\lambda= \pm \frac{1}{2}} \chi_{\lambda} \int_{\Lambda}^{e^{\delta \curlyvee} \Lambda} \frac{d k^{+}}{2 \pi} \int \frac{d^{2} \mathbf{k}}{(2 \pi)^{2}} \frac{1}{\sqrt{2}}\left(b_{\lambda}^{\alpha}\left(k^{+}, \mathbf{k}\right) e^{-i k \cdot x}+d_{\lambda}^{\alpha \dagger}\left(k^{+}, \mathbf{k}\right) e^{i k \cdot x}\right),
$$

and

$$
\bar{\psi}_{+}^{\alpha}(x)=\sum_{\lambda= \pm \frac{1}{2}} \chi_{\lambda} \int_{e^{\delta \curlyvee}}^{\infty} \frac{d k^{+}}{2 \pi} \int \frac{d^{2} \mathbf{k}}{(2 \pi)^{2}} \frac{1}{\sqrt{2}}\left(b_{\lambda}^{\alpha}\left(k^{+}, \mathbf{k}\right) e^{-i k \cdot x}+d_{\lambda}^{\alpha \dagger}\left(k^{+}, \mathbf{k}\right) e^{i k \cdot x}\right) .
$$

After inserting $A_{i}^{a}(x)=\underline{A}_{i}^{a}(x)+\bar{A}_{i}^{a}(x)$ and $\psi_{+}^{\alpha}(x)=\underline{\psi}_{+}^{\alpha}(x)+\bar{\psi}_{+}^{\alpha}(x)$ to $H_{\text {int }}$ defined in (2.5), we define the following components of the interaction Hamiltonian:

$$
\begin{aligned}
H_{g} \equiv & -g \int d x^{-} d^{2} \mathbf{x}\left(\partial_{i} \underline{A}_{i}^{a}\right)\left(f^{a b c} \frac{1}{\partial^{+}}\left(\bar{A}_{j}^{b} \partial^{+} \bar{A}_{j}^{c}\right)+2 \frac{1}{\partial^{+}}\left(\bar{\psi}_{+}^{\dagger} t^{a} \bar{\psi}_{+}\right)\right) \\
H_{g q q} \equiv & -g \int d x^{-} d^{2} \mathbf{x}\left(2\left(\partial_{i} \underline{A}_{i}^{a}\right) \frac{1}{\partial^{+}}\left(\underline{\psi}_{+}^{\dagger} t^{a} \underline{\psi}_{+}\right)+\underline{\psi}_{+}^{\dagger} t^{a}\left(\sigma_{i} \partial_{i}\right) \frac{1}{\partial^{+}}\left(\sigma_{j} \underline{A}_{j}^{a} \underline{\psi}_{+}\right)\right. \\
& \left.+\underline{\psi}_{+}^{\dagger} t^{a}\left(\sigma_{i} \underline{A}_{i}^{a}\right) \frac{1}{\partial^{+}}\left(\sigma_{j} \partial_{j} \underline{\psi}_{+}\right)\right) \\
H_{g g g} \equiv & -g f^{a b c} \int d x^{-} d^{2} \mathbf{x}\left(\left(\partial_{i} \underline{A}_{j}^{a}\right) \underline{A}_{i}^{b} \underline{A}_{j}^{c}+\left(\partial_{i} \underline{A}_{i}^{a}\right) \frac{1}{\partial^{+}}\left(\underline{A}_{j}^{b} \partial^{+} \underline{A}_{j}^{c}\right)\right), \\
H_{q q-\text { inst }} \equiv & 2 g^{2} \int d x^{-} d^{2} \mathbf{x} \frac{1}{\partial^{+}}\left(\underline{\psi}_{+}^{\dagger} t^{a} \underline{\psi}_{+}\right)\left(f^{a b c} \frac{1}{\partial^{+}}\left(\bar{A}_{j}^{b} \partial^{+} \bar{A}_{j}^{c}\right)+2 \frac{1}{\partial^{+}}\left(\bar{\psi}_{+}^{\dagger} t^{a} \bar{\psi}_{+}\right)\right), \\
H_{g g-\text { inst }} \equiv & g^{2} f^{a b c} \int d x^{-} d^{2} \mathbf{x} \frac{1}{\partial^{+}}\left(\underline{A}_{i}^{b} \partial^{+} \underline{A}_{i}^{c}\right)\left(f^{a d e} \frac{1}{\partial^{+}}\left(\bar{A}_{j}^{d} \partial^{+} \bar{A}_{j}^{e}\right)+2 \frac{1}{\partial^{+}}\left(\bar{\psi}_{+}^{\dagger} t^{a} \bar{\psi}_{+}\right)\right), \\
H_{g g g g} \equiv & \frac{g^{2}}{4} f^{a b c} f^{a d e} \int d x^{-} d^{2} \mathbf{x}\left(\underline{A}_{i}^{b} \underline{A}_{j}^{c} \underline{A}_{i}^{d} \underline{A}_{j}^{e}-2 \frac{1}{\partial^{+}}\left(\underline{A}_{i}^{b} \partial^{+} \underline{A}_{i}^{c}\right) \frac{1}{\partial^{+}}\left(\underline{A}_{j}^{d} \partial^{+} \underline{A}_{j}^{e}\right)\right), \\
H_{g g q q} \equiv & -i g^{2} \int d x^{-} d^{2} \mathbf{x} \underline{\psi}_{+}^{\dagger} t^{a} t^{b} \sigma_{i} \underline{A}_{i}^{a} \frac{1}{\partial^{+}}\left(\sigma_{j} \underline{A}_{j}^{b} \underline{\psi}_{+}\right), \\
H_{g q} \equiv & -g \int d x^{-} d^{2} \mathbf{x}\left(\bar{\psi}_{+}^{\dagger} t^{a}\left(\sigma_{i} \partial_{i}\right) \frac{1}{\partial^{+}}\left(\sigma_{j} \underline{A}_{j}^{a} \underline{\psi}_{+}\right)+\bar{\psi}_{+}^{\dagger} t^{a}\left(\sigma_{i} \underline{A}_{i}^{a}\right) \frac{1}{\partial^{+}}\left(\sigma_{j} \partial_{j} \underline{\psi}_{+}\right)\right. \\
& \left.+i g \bar{\psi}_{+}^{\dagger} t^{a} t^{b} \sigma_{i} \underline{A}_{i}^{a} \frac{1}{\partial^{+}}\left(\sigma_{j} \underline{A}_{j}^{b} \underline{\psi}_{+}\right)\right) .
\end{aligned}
$$


The eikonal approximation means that all contributions involving division by large (valence) longitudinal momenta are neglected, such as $\frac{1}{\partial^{+}}\left(\underline{\psi}_{+}^{\dagger} t^{a} \bar{\psi}_{+}\right) \sim \frac{1}{\partial^{+}}\left(\underline{A}_{j}^{b} \partial^{+} \bar{A}_{j}^{c}\right) \sim 0$. Furthermore, we only keep terms which are relevant for our NLO calculation, the ones that contain either interaction between soft modes or interaction between soft and valence modes. Some soft interaction terms that would contribute starting from NNLO only, such as instantaneous gluon-quark interaction term, as well as the valence Hamiltonian are not written down explicitly.

\section{Integrals and Fourier transformations}

In the following appendix we assemble all the integrals that were used throughout our calculations in this paper.

$$
\begin{aligned}
& \int_{0}^{1} d \xi\left(4 \xi^{2}-4 \xi+2\right)(\ln (\xi(1-\xi))-1)=-\frac{38}{9} . \\
& \int_{0}^{1} d \xi \frac{1}{(1-\xi)\left(X^{\prime}\right)^{2}+\xi X^{2}}=\frac{1}{X^{2}-\left(X^{\prime}\right)^{2}} \ln \left(\frac{X^{2}}{\left(X^{\prime}\right)^{2}}\right) . \\
& \int_{0}^{1} d \xi \frac{1}{\left((1-\xi)\left(X^{\prime}\right)^{2}+\xi X^{2}\right)\left((1-\xi)\left(Y^{\prime}\right)^{2}+\xi Y^{2}\right)}=\frac{1}{\left(X^{\prime}\right)^{2} Y^{2}-X^{2}\left(Y^{\prime}\right)^{2}} \ln \left(\frac{\left(X^{\prime}\right)^{2} Y^{2}}{X^{2}\left(Y^{\prime}\right)^{2}}\right) \\
& \int_{0}^{1} d \xi \frac{1}{\left((1-\xi)\left(X^{\prime}\right)^{2}+\xi X^{2}\right)\left((1-\xi)\left(Y^{\prime}\right)^{2}+\xi Y^{2}\right)}=-\frac{1}{\left(\left(X^{\prime}\right)^{2}-X^{2}\right)\left(\left(Y^{\prime}\right)^{2}-Y^{2}\right)} \\
& +\frac{\left(X^{\prime}\right)^{2} X^{2}}{\left(\left(X^{\prime}\right)^{2}-X^{2}\right)^{2}\left(\left(X^{\prime}\right)^{2} Y^{2}-X^{2}\left(Y^{\prime}\right)^{2}\right)} \ln \left(\frac{X^{2}}{\left(X^{\prime}\right)^{2}}\right) \\
& +\frac{\left(Y^{\prime}\right)^{2} Y^{2}}{\left(\left(Y^{\prime}\right)^{2}-Y\right)^{2}\left(\left(X^{\prime}\right)^{2} Y^{2}-X^{2}\left(Y^{\prime}\right)^{2}\right)} \ln \left(\frac{Y^{2}}{\left(Y^{\prime}\right)^{2}}\right) .
\end{aligned}
$$

The integrals below are evaluated under the assumption $\Lambda \rightarrow 0$ and $\delta \mathrm{Y} \rightarrow \infty$. While explicitly divergent terms will be retain, we will take the limit in all the non-divergent terms without further notification.

$$
\begin{aligned}
& \int_{\frac{\Lambda}{k^{+}}}^{1-\frac{\Lambda}{k^{+}}} d \xi \frac{1}{\xi\left((1-\xi)\left(W^{\prime}\right)^{2}+\xi W^{2}\right)}=-\frac{1}{\left(W^{\prime}\right)^{2}}\left(\ln \left(\frac{\Lambda}{k^{+}}\right)+\ln \left(\frac{W^{2}}{\left(W^{\prime}\right)^{2}}\right)\right) . \\
& \int_{\frac{\Lambda}{k^{+}}}^{1-\frac{\Lambda}{k^{+}}} d \xi \frac{1}{(1-\xi)\left((1-\xi)\left(W^{\prime}\right)^{2}+\xi W^{2}\right)}=-\frac{1}{W^{2}}\left(\ln \left(\frac{\Lambda}{k^{+}}\right)+\ln \left(\frac{W^{2}}{\left(W^{\prime}\right)^{2}}\right)\right) . \\
& \int_{\frac{\Lambda}{k^{+}}}^{1-\frac{\Lambda}{k^{+}}} d \xi \frac{1+\xi}{\xi(1-\xi)^{2}}=\frac{2 k^{+}}{\Lambda}-2-2 \ln \left(\frac{\Lambda}{k^{+}}\right) . \\
& \int_{\frac{\Lambda}{k^{+}}}^{1-\frac{\Lambda}{k^{+}}} d \xi \frac{1+\xi}{(1-\xi)^{2}}=\frac{2 k^{+}}{\Lambda}-2+\ln \left(\frac{\Lambda}{k^{+}}\right) . \\
& \int_{\frac{\Lambda}{k^{+}}}^{\frac{e^{\delta \Upsilon}}{k^{+}}} d \xi \frac{1}{\xi} \ln \xi=\frac{1}{2} \ln ^{2}\left(\frac{e^{\delta \curlyvee} \Lambda}{k^{+}}\right)-\frac{1}{2} \ln ^{2}\left(\frac{\Lambda}{k^{+}}\right) .
\end{aligned}
$$




$$
\begin{aligned}
& \int_{\frac{\Lambda}{k^{+}}}^{1-\frac{\Lambda}{k^{+}}} d \xi\left(\xi(1-\xi)-2+\frac{1}{\xi(1-\xi)}\right) \ln \left(\frac{\mathbf{k}^{2} \xi(1-\xi)}{\mu_{\overline{M S}}^{2}}\right)= \\
& {\left[-\frac{11}{6}-2 \ln \left(\frac{\Lambda}{k^{+}}\right)\right] \ln \left(\frac{\mathbf{k}^{2}}{\mu_{\overline{M S}}^{2}}\right)-\ln ^{2}\left(\frac{\Lambda}{k^{+}}\right)+\frac{67}{18}-\frac{\pi^{2}}{3} .} \\
& \int_{\frac{\Lambda}{k^{+}}}^{1-\frac{\Lambda}{k^{+}}} d \xi \frac{(\xi-2) \ln (\xi)+(1+\xi) \ln (1-\xi)}{\xi(1-\xi)}=0 . \\
& \int_{\frac{\Lambda}{k^{+}}}^{1-\frac{\Lambda}{k^{+}}} d \xi \frac{(\xi-2) \ln \xi-(1+\xi) \ln (1-\xi)}{\xi(1-\xi)}=\frac{\pi^{2}}{3}+2 \ln ^{2}\left(\frac{\Lambda}{k^{+}}\right) . \\
& \int_{\frac{\Lambda}{k^{+}}}^{1-\frac{\Lambda}{k^{+}}} d \xi \frac{1+\xi}{\xi(1-\xi)}=-\int_{\frac{\Lambda}{k^{+}}}^{1-\frac{\Lambda}{k^{+}}} d \xi \frac{\xi-2}{\xi(1-\xi)}=-3 \ln \left(\frac{\Lambda}{k^{+}}\right) . \\
& \int_{\frac{\Lambda}{k^{+}}}^{1-\frac{\Lambda}{k^{+}}} d \xi \frac{\xi-2}{\xi^{2}(1-\xi)}=2\left(1-\frac{k^{+}}{\Lambda}+\ln \left(\frac{\Lambda}{k^{+}}\right)\right) \text {. } \\
& \int_{\Lambda}^{\Lambda e^{\delta \curlyvee}} \frac{d k^{+}}{k^{+}}\left(\ln ^{2}\left(\frac{\Lambda e^{\delta Y}}{k^{+}}\right)-\ln ^{2}\left(\frac{\Lambda}{k^{+}}\right)\right)=0 . \\
& \int_{1-\frac{\Lambda}{k^{+}}}^{\frac{e^{\delta Y_{\Lambda}}}{k^{+}}} \frac{b+a \xi}{\xi(1-\xi)(c+d(\xi-1))} d \xi=\left(\frac{a}{c}-\frac{b d}{c(c-d)}\right)\left[\ln \left(c-d+\frac{e^{\delta \mathrm{Y}} \Lambda}{k^{+}} d\right)\right. \\
& \left.-\ln \left(c-\frac{\Lambda}{k^{+}} d\right)\right]-\frac{a+b}{c}\left[\ln \left(1-\frac{e^{\delta \mathrm{Y}} \Lambda}{k^{+}}\right)-\ln \left(\frac{\Lambda}{k^{+}}\right)\right]+\frac{b}{c-d} \ln \left(\frac{e^{\delta \mathrm{Y}} \Lambda}{k^{+}}\right) . \\
& \int_{\frac{\Lambda}{k^{+}}}^{\frac{e^{\delta \Upsilon_{\Lambda}}}{k^{+}}} d \xi \frac{1}{\xi\left(\xi+\frac{\mathbf{p}^{2}}{\mathbf{k}^{2}}\right)}=\frac{\mathbf{k}^{2}}{\mathbf{p}^{2}}\left(\ln \left(\frac{\Lambda}{k^{+}}\right)-\ln \left(\frac{\mathbf{p}^{2}}{\mathbf{k}^{2}}\right)\right) \text {. } \\
& \int_{\frac{\Lambda}{k^{+}}}^{1-\frac{\Lambda}{k^{+}}} d \xi \frac{\xi}{\left(\xi(\mathbf{k}-\mathbf{p})^{2}+(1-\xi) \mathbf{k}^{2}\right)(1-\xi)}= \\
& \frac{\mathbf{k}^{2}}{(\mathbf{k}-\mathbf{p})^{2}\left(\mathbf{k}^{2}-(\mathbf{k}-\mathbf{p})^{2}\right)} \ln \left(\frac{(\mathbf{k}-\mathbf{p})^{2}}{\mathbf{k}^{2}}\right)-\frac{1}{(\mathbf{k}-\mathbf{p})^{2}} \ln \left(\frac{\Lambda}{k^{+}}\right) \text {. } \\
& \int_{\frac{\Lambda}{k^{+}}}^{1-\frac{\Lambda}{k^{+}}} d \xi \frac{1}{\left(\xi(\mathbf{k}-\mathbf{p})^{2}+(1-\xi) \mathbf{k}^{2}\right)(1-\xi)}=\frac{1}{(\mathbf{k}-\mathbf{p})^{2}} \ln \left(\frac{(\mathbf{k}-\mathbf{p})^{2}}{\mathbf{k}^{2}}\right)-\frac{1}{(\mathbf{k}-\mathbf{p})^{2}} \ln \left(\frac{\Lambda}{k^{+}}\right) \text {. }
\end{aligned}
$$

- Fourier transforms. Here we present a list of the integrals necessary to perform the Fourier transformations during the computations. A comprehensive list of Fourier transforms can be found in [93].

$$
\begin{aligned}
& \frac{1}{(2 \pi)^{3}} \int d x^{-} d^{2} \mathbf{x} e^{i(k-p) x}=\delta^{(3)}(k-p) . \\
& \int \frac{d^{2} \mathbf{k}}{2 \pi} \frac{\mathbf{k}^{i}}{\mathbf{k}^{2}} e^{i \mathbf{k} \cdot X}=\frac{i X^{i}}{X^{2}} . \\
& \int \frac{d^{2} \mathbf{k}}{2 \pi} \frac{\mathbf{k}^{i}}{\mathbf{k}^{2}} \ln \left(\frac{\mathbf{k}^{2}}{\mu^{2}}\right) e^{i \mathbf{k} \cdot X}=\frac{i X^{i}}{X^{2}}\left(-2 \gamma-\ln \left(\frac{X^{2} \mu^{2}}{4}\right)\right) .
\end{aligned}
$$




$$
\begin{aligned}
& \int \frac{d^{2} \mathbf{k}}{2 \pi} \frac{d^{2} \mathbf{p}}{2 \pi} \frac{1}{\xi(1-\xi) \mathbf{k}^{2}+\mathbf{p}^{2}} e^{i \mathbf{k} \cdot X+i \mathbf{p} \cdot Z}=\frac{1}{X^{2}+\xi(1-\xi) Z^{2}} . \\
& \int \frac{d^{2} \mathbf{k}}{2 \pi} \frac{d^{2} \mathbf{p}}{2 \pi} \frac{\mathbf{k}^{i} \mathbf{p}^{j}}{\mathbf{k}^{2}\left(a \mathbf{k}^{2}+b \mathbf{p}^{2}\right)} e^{i \mathbf{k} \cdot X+i \mathbf{p} \cdot Z}=-\frac{X^{i} Z^{j}}{Z^{2}\left(b X^{2}+a Z^{2}\right)} . \\
& \int \frac{d^{2} k}{2 \pi} \frac{d^{2} p}{2 \pi} \frac{\mathbf{k} \cdot \mathbf{p}}{\mathbf{k}^{2} \mathbf{p}^{2}} \ln \left(\frac{(\mathbf{k}-\mathbf{p})^{2}}{\mu_{\overline{M S}}^{2}}\right) e^{i \mathbf{k} \cdot X-i \mathbf{p} \cdot Y}=\frac{X \cdot Y}{X^{2} Y^{2}}\left(\ln \left(\frac{(X-Y)^{2}}{X^{2} Y^{2} \mu_{\overline{M S}}^{2}}\right)-2 \gamma+\log 4\right) . \\
& \int \frac{d^{2} \mathbf{k}}{2 \pi} \frac{d^{2} \mathbf{p}}{2 \pi} \frac{1}{\mathbf{p}^{2}} \ln \left(\frac{(\mathbf{k}-\mathbf{p})^{2}}{\mathbf{k}^{2}}\right) e^{-i \mathbf{k} \cdot X+i \mathbf{p} \cdot Y}=\frac{1}{X^{2}} \ln \left(\frac{(X-Y)^{2}}{Y^{2}}\right) \cdot 4 \\
& \int \frac{d^{2} \mathbf{k}}{2 \pi} \frac{d^{2} \mathbf{p}}{2 \pi}\left(\frac{\mathbf{k}^{i}-\mathbf{p}^{i}}{\mathbf{p}^{2}(\mathbf{k}-\mathbf{p})^{2}}-\frac{\mathbf{k}^{i}}{\mathbf{k}^{2} \mathbf{p}^{2}}+\frac{\mathbf{k}^{i}}{\mathbf{k}^{2}(\mathbf{k}-\mathbf{p})^{2}}\right) \ln \left(\frac{\mathbf{p}^{2}}{\mathbf{k}^{2}}\right) e^{-i \mathbf{k} \cdot Y-i \mathbf{p} \cdot(X-Y)}-(X \leftrightarrow Y) \\
& =-\frac{i}{2}\left(\frac{X^{i}}{X^{2}}-\frac{Y^{i}}{Y^{2}}\right) \ln \left(\frac{X^{2}}{(X-Y)^{2}}\right) \ln \left(\frac{Y^{2}}{(X-Y)^{2}}\right) \text {. } \\
& \int d^{2} \mathbf{k} d^{2} \mathbf{p} e^{-i(\mathbf{k}-\mathbf{p}) \cdot \mathbf{y}-i \mathbf{p} \cdot \mathbf{x}+i \mathbf{k} \cdot \mathbf{z}} \frac{\mathbf{k}^{i}}{\mathbf{k}^{2} \mathbf{p}^{2}}=2 i \pi \int_{\mathbf{z}^{\prime}} \frac{X^{\prime} \cdot Y^{\prime} Y^{i}}{\left(X^{\prime}\right)^{2}\left(Y^{\prime}\right)^{2} Y^{2}} . \\
& \int d^{2} \mathbf{k} d^{2} \mathbf{p} e^{-i(\mathbf{k}-\mathbf{p}) \cdot \mathbf{x}-i \mathbf{p} \cdot \mathbf{y}+i \mathbf{k} \cdot \mathbf{z}} \frac{\mathbf{p}^{i}}{\mathbf{p}^{2}(\mathbf{k}-\mathbf{p})^{2}}=2 i \pi \int_{\mathbf{z}^{\prime}} \frac{X^{\prime} \cdot Z Y^{i}}{\left(X^{\prime}\right)^{2} Z^{2} Y^{2}} . \\
& \int d^{2} \mathbf{k} d^{2} \mathbf{p} e^{-i(\mathbf{k}-\mathbf{p}) \cdot \mathbf{x}-i \mathbf{p} \cdot \mathbf{y}+i \mathbf{k} \cdot \mathbf{z}} \frac{\mathbf{p} \cdot(\mathbf{p}-\mathbf{k}) \mathbf{k}^{i}}{\mathbf{k}^{2} \mathbf{p}^{2}(\mathbf{k}-\mathbf{p})^{2}}=2 i \pi \int_{\mathbf{z}^{\prime}} \frac{X^{\prime} \cdot Y^{\prime} Z^{i}}{\left(X^{\prime}\right)^{2}\left(Y^{\prime}\right)^{2} Z^{2}} .
\end{aligned}
$$

- Dimensional regularization.

$$
\begin{aligned}
& \int \frac{d^{d} \mathbf{k}}{(2 \pi)^{d}} \frac{\mathbf{k}^{2}}{\left(\mathbf{k}^{2}+\Delta\right)^{n}}=\frac{d}{2(4 \pi)^{d / 2}} \frac{\Gamma\left(n-1-\frac{d}{2}\right)}{\Gamma(n)}\left(\frac{1}{\Delta}\right)^{n-1-\frac{d}{2}} \\
& \int \frac{d^{d} \mathbf{k}}{(2 \pi)^{d}} \frac{1}{\left(\mathbf{k}^{2}+\Delta\right)^{n}}=\frac{1}{(4 \pi)^{d / 2}} \frac{\Gamma\left(n-\frac{d}{2}\right)}{\Gamma(n)}\left(\frac{1}{\Delta}\right)^{n-\frac{d}{2}} .
\end{aligned}
$$

$\Gamma$ function expansion:

$$
\Gamma(-n+\epsilon)=\frac{(-1)^{n}}{n !}\left[\frac{1}{\epsilon}+\sum_{k=1}^{k=n} \frac{1}{k}-\gamma\right]+\mathcal{O}(\epsilon) .
$$

\section{Properties of the NLO JIMWLK kernels}

Here we quote from [57] some useful properties of the kernels defined in section 2.8:

$$
\begin{aligned}
\int_{\mathbf{z}^{\prime}} K_{\text {JJSSJ }}\left(\mathbf{y}, \mathbf{x}, \mathbf{y}, \mathbf{z}, \mathbf{z}^{\prime}\right) & =0 . \\
\int_{\mathbf{z}} K_{\text {JJSJ }}(\mathbf{y}, \mathbf{x}, \mathbf{y}, \mathbf{z}) & =\int_{\mathbf{z}, \mathbf{z}^{\prime}} K_{\text {JJSSJ }}\left(\mathbf{y}, \mathbf{y}, \mathbf{x}, \mathbf{z}, \mathbf{z}^{\prime}\right)=0 . \\
\int_{\mathbf{z}, \mathbf{z}^{\prime}} \widetilde{K}\left(\mathbf{x}, \mathbf{y}, \mathbf{z}, \mathbf{z}^{\prime}\right) & =i \int_{\mathbf{z}}\left[K_{\text {JJSJ }}(\mathbf{y}, \mathbf{x}, \mathbf{y}, \mathbf{z})+K_{\text {JJSJ }}(\mathbf{x}, \mathbf{y}, \mathbf{x}, \mathbf{z})\right]=0 . \\
K_{\text {JJSJ }}(\mathbf{w}, \mathbf{x}, \mathbf{y}, \mathbf{z}) & =\int_{\mathbf{z}^{\prime}}\left[K_{\text {JJSSJ }}\left(\mathbf{y}, \mathbf{w}, \mathbf{x}, \mathbf{z}, \mathbf{z}^{\prime}\right)-K_{\text {JJSSJ }}\left(\mathbf{x}, \mathbf{w}, \mathbf{y}, \mathbf{z}, \mathbf{z}^{\prime}\right)\right] .
\end{aligned}
$$


Throughout the calculation, we encounter the kernels $K_{q q}$ and $K_{\text {JSSJ }}$ integrated over $z^{\prime}$. These integrals are UV divergent. In order to evaluate them, they have to be written in their momentum space representation and dimensionally regularised. From $[52,53]$ and using (C.25), (C.26),

$$
\begin{aligned}
& \int_{\mathbf{z}^{\prime}} K_{q \bar{q}}\left(\mathbf{x}, \mathbf{y}, \mathbf{z}, \mathbf{z}^{\prime}\right)=-\frac{\alpha_{s}^{2} N_{f}}{12 \pi^{3}}\left(\frac{1}{X^{2}} \ln \left(X^{2} \mu_{\overline{M S}}^{2}\right)+\frac{1}{Y^{2}} \ln \left(Y^{2} \mu_{\overline{M S}}^{2}\right)\right. \\
& \left.+\frac{(X-Y)^{2}}{X^{2} Y^{2}} \ln \left(\frac{(X-Y)^{2}}{X^{2} Y^{2} \mu_{\overline{M S}}^{2}}\right)+\frac{X \cdot Y}{X^{2} Y^{2}}\left(\frac{10}{3}+4 \gamma-2 \ln 4\right)\right) .
\end{aligned}
$$

From [47],

$$
\begin{array}{r}
\int_{\mathbf{z}^{\prime}} K_{\text {JSSJ }}\left(\mathbf{x}, \mathbf{y}, \mathbf{z}, \mathbf{z}^{\prime}\right)=\frac{\alpha_{s}^{2}}{4 \pi^{3}}\left(\frac{11}{6 X^{2}} \ln \left(X^{2} \mu \frac{2}{M S}\right)+\frac{11}{6 Y^{2}} \ln \left(Y^{2} \mu \frac{2}{M S}\right)+\frac{11(X-Y)^{2}}{6 X^{2} Y^{2}}\right. \\
\left.\quad \times \ln \left(\frac{(X-Y)^{2}}{X^{2} Y^{2} \mu_{\frac{M S}{2}}^{2}}\right)+\frac{X \cdot Y}{X^{2} Y^{2}}\left(\frac{67}{9}-\frac{\pi^{2}}{3}+\frac{11}{3}[2 \gamma-\ln 4]\right)\right)+\int_{\mathbf{z}^{\prime}} \widetilde{K}\left(\mathbf{x}, \mathbf{y}, \mathbf{z}, \mathbf{z}^{\prime}\right) .
\end{array}
$$

\section{E Perturbation theory with non-commutative matrix elements}

In this appendix we briefly sketch the derivation of the wave function up to order $g^{3}$. Our prime goal is to provide a derivation, which would be valid for operator valued noncommuting matrix elements. We begin with an unperturbed Hamiltonian $H_{0} . E_{n}^{(0)}$ and $\left|n^{(0)}\right\rangle$ denote the eigenvalues and the eigenstates of $H_{0}$. Let introduce a weak perturbation $H_{\text {int }}$. The energy levels and eigenstates of the perturbed Hamiltonian are $E_{n}$, and $|n\rangle$ :

$$
\begin{array}{ll}
H_{0}\left|n^{(0)}\right\rangle=E_{n}^{(0)}\left|n^{(0)}\right\rangle ; & \left(H_{0}+H_{\mathrm{int}}\right)|n\rangle=E_{n}|n\rangle . \\
E_{n}=E_{n}^{(0)}+E_{n}^{(1)}+E_{n}^{(2)}+\ldots & |n\rangle=\left|n^{(0)}\right\rangle+\left|n^{(1)}\right\rangle+\left|n^{(2)}\right\rangle+\ldots
\end{array}
$$

Where $\left|n^{(i)}\right\rangle$ are orthogonal states, corresponding to $i$-th order in perturbation theory. By inserting (E.2) in (E.1), expanding and equating terms in the same order of $g$, we arrive at:

$$
\begin{aligned}
& |n\rangle=\left|n^{(0)}\right\rangle-\sum_{i \neq 0} \frac{1}{E_{i}^{(0)}-E_{0}^{(0)}-E_{i}^{(1)}-E_{i}^{(2)}}\left|n^{(i)}\right\rangle\left\langle n^{(i)}\left|H_{\mathrm{int}}\right| n^{(0)}\right\rangle \\
& +\sum_{i, j \neq 0} \frac{1}{E_{i}^{(0)}-E_{0}^{(0)}-E_{i}^{(1)}}\left|n^{(i)}\right\rangle\left\langle n^{(i)}\left|H_{\mathrm{int}}\right| n^{(j)}\right\rangle \frac{1}{E_{j}^{(0)}-E_{0}^{(0)}-E_{j}^{(1)}}\left\langle n^{(j)}\left|H_{\mathrm{int}}\right| n^{(0)}\right\rangle \\
& -\sum_{i, j, k \neq 0} \frac{1}{E_{i}^{(0)}-E_{0}^{(0)}}\left|n^{(i)}\right\rangle\left\langle n^{(i)}\left|H_{\mathrm{int}}\right| n^{(j)}\right\rangle \frac{1}{E_{j}^{(0)}-E_{0}^{(0)}}\left\langle n^{(j)}\left|H_{\mathrm{int}}\right| n^{(k)}\right\rangle \\
& \times \frac{1}{E_{k}^{(0)}-E_{0}^{(0)}}\left\langle n^{(k)}\left|H_{\mathrm{int}}\right| n^{(0)}\right\rangle,
\end{aligned}
$$

with

$$
E_{i}^{(1)} \equiv\left\langle n^{(i)}\left|H_{\mathrm{int}}\right| n^{(i)}\right\rangle \quad E_{i}^{(2)} \equiv-\sum_{i \neq 0} \frac{\left|\left\langle n^{(i)}\left|H_{\mathrm{int}}\right| 0\right\rangle\right|^{2}}{E_{i}^{(0)}}
$$


Let's focus on the vacuum state to be denoted $|\psi\rangle_{N}$ below. We set $E_{0}^{(0)}=0$. Having in mind $H_{\text {int }}$ that does not have a matrix element over the vacuum state, from (E.4), $E_{n}^{(1)}=0$. The denominator in the first line of (E.3):

$$
\frac{1}{E_{i}^{(0)}-E_{0}^{(0)}-E_{i}^{(1)}-E_{i}^{(2)}} \approx \frac{1}{E_{i}^{(0)}}-\frac{1}{\left(E_{i}^{(0)}\right)^{2}} \sum_{j \neq 0} \frac{\left|\left\langle n^{(j)}\left|H_{\mathrm{int}}\right| 0\right\rangle\right|^{2}}{E_{j}^{(0)}} .
$$

Substituting the last relation in (E.3), we finally get:

$$
\begin{aligned}
|\psi\rangle_{N}= & |0\rangle-\left|n^{(i)}\right\rangle \frac{\left\langle n^{(i)}\left|H_{\mathrm{int}}\right| 0\right\rangle}{E_{i}^{(0)}}+\left|n^{(i)}\right\rangle \frac{\left\langle n^{(i)}\left|H_{\mathrm{int}}\right| n^{(j)}\right\rangle\left\langle n^{(j)}\left|H_{\mathrm{int}}\right| 0\right\rangle}{E_{i}^{(0)} E_{j}^{(0)}} \\
& -\left|n^{(i)}\right\rangle \frac{\left\langle n^{(i)}\left|H_{\mathrm{int}}\right| n^{(j)}\right\rangle\left\langle n^{(j)}\left|H_{\mathrm{int}}\right| n^{(k)}\right\rangle\left\langle n^{(k)}\left|H_{\mathrm{int}}\right| 0\right\rangle}{E_{i}^{(0)} E_{j}^{(0)} E_{k}^{(0)}} \\
& +\left|n^{(i)}\right\rangle \frac{\left|\left\langle n^{(j)}\left|H_{\mathrm{int}}\right| 0\right\rangle\right|^{2}\left\langle n^{(i)}\left|H_{\mathrm{int}}\right| 0\right\rangle}{\left(E_{i}^{(0)}\right)^{2} E_{j}^{(0)}} .
\end{aligned}
$$

\section{F The phase of the wave function}

The condition (3.31) imposed in order to find the phase reads

$$
\left\langle\psi^{\mathrm{NLO}}\left|\frac{\delta}{\delta \rho^{d}(\mathbf{w})}\right| \psi^{\mathrm{NLO}}\right\rangle=0 .
$$

It is trivial to check that the LO LCWF satisfies (F.1). This happens automatically thanks to cancelation between contributions of normalisation against diagonal contribution from one gluon component. Similar cancelations happen when $\left|\psi^{\mathrm{NLO}}\right\rangle$ (3.84) is inserted in (F.1). What we are left with are the "interference" contributions between different components of the LCWF and the phase:

$$
\begin{aligned}
& \left\langle\psi^{\mathrm{NLO}}\left|\frac{\delta}{\delta \rho^{d}(\mathbf{w})}\right| \psi^{\mathrm{NLO}}\right\rangle=\left\langle\psi_{g g \rho}\left|\frac{\delta}{\delta \rho^{d}(\mathbf{w})}\right| \psi_{g g \rho \rho}\right\rangle+\left\langle\psi_{g g \rho \rho}\left|\frac{\delta}{\delta \rho^{d}(\mathbf{w})}\right| \psi_{g g \rho}\right\rangle \\
& +\left\langle\psi_{g \rho \rho}\left|\frac{\delta}{\delta \rho^{d}(\mathbf{w})}\right| \psi_{g \rho}^{L O}\right\rangle+\left\langle\psi_{g \rho}^{L O}\left|\frac{\delta}{\delta \rho^{d}(\mathbf{w})}\right| \psi_{g \rho \rho}\right\rangle+\left\langle 0\left|\frac{\delta}{\delta \rho^{d}(\mathbf{w})} i \phi^{\mathrm{NLO}}\right| 0\right\rangle=0
\end{aligned}
$$

After insertion of expressions (3.4), (3.94), (3.96), (3.98), (3.100), the condition (F.1) becomes:

$$
\begin{aligned}
- & \delta \mathbf{Y} \int_{\mathbf{x}, \mathbf{y}, \mathbf{z}, \mathbf{z}^{\prime}} f^{d c b} \rho^{b}(\mathbf{y}) \rho^{c}(\mathbf{x})\left[-K_{\mathrm{JJSSJ}}\left(\mathbf{w}, \mathbf{x}, \mathbf{y}, \mathbf{z}, \mathbf{z}^{\prime}\right)+K_{\mathrm{JJSSJ}}\left(\mathbf{x}, \mathbf{w}, \mathbf{y}, \mathbf{z}, \mathbf{z}^{\prime}\right)\right. \\
& \left.-K_{\mathrm{JJSSJ}}\left(\mathbf{y}, \mathbf{w}, \mathbf{x}, \mathbf{z}, \mathbf{z}^{\prime}\right)\right]+\left\langle 0\left|\frac{\delta}{\delta \rho^{d}(\mathbf{w})} i \phi^{\mathrm{NLO}}\right| 0\right\rangle=0 .
\end{aligned}
$$

In (F.3), the kernels $K_{\text {JJSSJ }}$ and $K_{\text {JJSJ }}$ originate from the overlaps $\left\langle\psi_{g g \rho \rho}\right| \psi_{g g} \rho$ and $\left\langle\psi_{g \rho \rho} \mid \psi_{g \rho}^{L O}\right\rangle$. The computation can be easily traced to a similar computation of $\Sigma_{\mathrm{vJJJ}}$ in section 4.7.

It is easy to see that the following ansatz for $i \phi^{\mathrm{NLO}}$ solves (F.3),

$$
i \phi^{\mathrm{NLO}}=-\delta \mathrm{Y} \int_{\mathbf{v}, \mathbf{x}, \mathbf{y}, \mathbf{z}, \mathbf{z}^{\prime}} K_{\mathrm{JJSSJ}}\left(\mathbf{v}, \mathbf{x}, \mathbf{y}, \mathbf{z}, \mathbf{z}^{\prime}\right) f^{a b c} \rho^{b}(\mathbf{y}) \rho^{a}(\mathbf{x}) \rho^{c}(\mathbf{v}) .
$$




\section{G Supplementary for section 3}

\section{G.1 Supplement for computation of $\left|\psi_{\boldsymbol{g}}^{\mathbf{1}}\right\rangle$}

$\left|\psi_{g}^{1}\right\rangle$ is defined in (3.49). The calculation in this section is identical to [52, 53]. After inserting the relevant matrix elements, (2.49) and (3.21):

$$
\begin{aligned}
\left|\psi_{g}^{1}\right\rangle= & -\sum_{f, \lambda_{1}, \lambda_{2}} \int_{\Lambda}^{e^{\delta \curlyvee} \Lambda} d k^{+} \int_{0}^{k^{+}} d p^{+} \int d^{2} \mathbf{k} d^{2} \mathbf{p} \frac{g^{3} \rho^{a}(-\mathbf{k}) \operatorname{tr}\left[t^{a} t^{d}\right] \mathbf{k}^{i}}{32 \pi^{9 / 2}\left(\frac{\mathbf{p}^{2}}{p^{+}}+\frac{(\mathbf{k}-\mathbf{p})^{2}}{\left(k^{+}-p^{+}\right)}\right) \mathbf{k}^{4} \sqrt{k^{+}}} \\
& \times \chi_{\lambda_{2}}^{\dagger}\left(\frac{2 \mathbf{k}^{j}}{k^{+}}-\sigma^{j} \frac{\sigma \cdot \mathbf{p}}{p^{+}}-\frac{\sigma \cdot(\mathbf{k}-\mathbf{p})}{k^{+}-p^{+}} \sigma^{j}\right) \chi_{\lambda_{1}} \\
& \times \chi_{\lambda_{1}}^{\dagger}\left(\frac{2 \mathbf{k}^{i}}{k^{+}}-\frac{\sigma \cdot \mathbf{p}}{p^{+}} \sigma^{i}-\sigma^{i} \frac{\sigma \cdot(\mathbf{k}-\mathbf{p})}{k^{+}-p^{+}}\right) \chi_{\lambda_{2}}\left|g_{j}^{d}(k)\right\rangle .
\end{aligned}
$$

By using

$$
\sum_{\lambda} \chi_{\lambda} \chi_{\lambda}^{\dagger}=\left(\begin{array}{ll}
1 & 0 \\
0 & 1
\end{array}\right)=I, \quad \sigma^{i} \sigma^{j}=i \varepsilon^{i j} \sigma^{3}+\delta^{i j} I, \quad \operatorname{tr}\left[t^{a} t^{d}\right]=\frac{1}{2} \delta^{a d},
$$

and

$$
\begin{aligned}
& \sum_{\lambda} \chi_{\lambda}^{\dagger}\left(\frac{2 \mathbf{k}^{j}}{k^{+}}-\sigma^{j} \frac{\sigma \cdot \mathbf{p}}{p^{+}}-\frac{\sigma \cdot(\mathbf{k}-\mathbf{p})}{k^{+}-p^{+}} \sigma^{j}\right)\left(\frac{2 \mathbf{k}^{i}}{k^{+}}-\sigma^{i} \frac{\sigma \cdot(\mathbf{k}-\mathbf{p})}{k^{+}-p^{+}}-\frac{\sigma \cdot \mathbf{p}}{p^{+}} \sigma^{i}\right) \chi_{\lambda} \\
&=\sum_{\lambda} \chi_{\lambda}^{\dagger}\left(4 \frac{\mathbf{k}^{i} \mathbf{k}^{j}}{\left(k^{+}\right)^{2}}-2 \frac{\mathbf{k}^{i} \mathbf{p}^{j}+\mathbf{k}^{j} \mathbf{p}^{i}}{k^{+} p^{+}}-2 \frac{\mathbf{k}^{i}\left(\mathbf{k}^{j}-\mathbf{p}^{j}\right)+\mathbf{k}^{j}\left(\mathbf{k}^{i}-\mathbf{p}^{i}\right)}{k^{+}\left(k^{+}-p^{+}\right)}+\frac{\mathbf{p}^{i} \mathbf{p}^{j}+\varepsilon^{i l} \varepsilon^{j k} \mathbf{p}^{k} \mathbf{p}^{l}}{\left(p^{+}\right)^{2}}\right. \\
& \quad+\frac{\mathbf{p}^{i}\left(\mathbf{k}^{j}-\mathbf{p}^{j}\right)+\mathbf{p}^{j}\left(\mathbf{k}^{i}-\mathbf{p}^{i}\right)-\varepsilon^{i l} \varepsilon^{j k}\left(\mathbf{k}^{k}-\mathbf{p}^{k}\right) \mathbf{p}^{l}-\varepsilon^{i l} \varepsilon^{j k}\left(\mathbf{k}^{l}-\mathbf{p}^{l}\right) \mathbf{p}^{k}}{p^{+}\left(k^{+}-p^{+}\right)} \\
&\left.\quad+\frac{\left(\mathbf{k}^{i}-\mathbf{p}^{i}\right)\left(\mathbf{k}^{j}-\mathbf{p}^{j}\right)+\varepsilon^{i l} \varepsilon^{j k}\left(\mathbf{k}^{i}-\mathbf{p}^{i}\right)\left(\mathbf{k}^{j}-\mathbf{p}^{j}\right)}{\left(k^{+}-p^{+}\right)^{2}}\right) \chi_{\lambda} \\
&= \frac{2 \widetilde{\mathbf{p}}^{i} \widetilde{\mathbf{p}}^{j}}{\left(k^{+}\right)^{2}}\left(\frac{1}{\xi^{2}}+\frac{1}{(1-\xi)^{2}}-\frac{2}{\xi(1-\xi)}\right)+\frac{2 \varepsilon^{i l} \varepsilon^{j k} \widetilde{\mathbf{p}}^{k} \widetilde{\mathbf{p}}^{l}}{\left(k^{+}\right)^{2}}\left(\frac{1}{\xi^{2}}+\frac{1}{(1-\xi)^{2}}+\frac{2}{\xi(1-\xi)}\right) \\
&= 2 \frac{\left(4 \xi^{2}-4 \xi+1\right) \widetilde{\mathbf{p}}^{i} \widetilde{\mathbf{p}}^{j}+\varepsilon^{i l} \varepsilon^{j k} \widetilde{\mathbf{p}}^{k} \widetilde{\mathbf{p}}^{l}}{\xi^{2}(1-\xi)^{2}\left(k^{+}\right)^{2}} .
\end{aligned}
$$

After change of variables according to (3.34) and (3.45):

$$
\begin{aligned}
\left|\psi_{g}^{1}\right\rangle= & -\int_{\Lambda}^{e^{\delta \curlyvee} \Lambda} d k^{+} \int_{0}^{1} d \xi \int d^{2} \mathbf{k} d^{2} \widetilde{\mathbf{p}} \\
& \times \frac{g^{3} N_{f} \rho^{a}(-\mathbf{k})\left(\left(4 \xi^{2}-4 \xi+1\right) \widetilde{\mathbf{p}}^{i} \widetilde{\mathbf{p}}^{j}+\varepsilon^{i l} \varepsilon^{j k} \widetilde{\mathbf{p}}^{k} \widetilde{\mathbf{p}}^{l}\right) \mathbf{k}^{i}}{32 \pi^{9 / 2} \xi(1-\xi) \mathbf{k}^{4}\left(\xi(1-\xi) \mathbf{k}^{2}+\widetilde{\mathbf{p}}^{2}\right) \sqrt{k^{+}}}\left|g_{j}^{a}(k)\right\rangle .
\end{aligned}
$$

The UV divergent $p$-integral is regularised via dimensional regularisation according to (3.28). Then, using (3.29) together with the following identity:

$$
\varepsilon^{i l} \varepsilon^{j k}=\delta^{i j} \delta^{k l}-\delta^{i k} \delta^{j l},
$$


we arrive at:

$$
\begin{aligned}
& \left|\psi_{g}^{1}\right\rangle \\
& =-\mu^{2 \epsilon} \int_{\Lambda}^{e^{\delta \curlyvee} \Lambda} d k^{+} \int \frac{d^{d} \mathbf{k}}{(2 \pi)^{d}} \frac{d^{d} \widetilde{\mathbf{p}}}{(2 \pi)^{d}} \int_{0}^{1} d \xi \frac{g^{3} N_{f} \rho^{a}(-\mathbf{k})\left(4 \xi^{2}-4 \xi+d\right) \widetilde{\mathbf{p}}^{2} \mathbf{k}^{i}}{2 \sqrt{\pi k^{+}} \mathbf{k}^{4}\left(\xi(1-\xi) \mathbf{k}^{2}+\widetilde{\mathbf{p}}^{2}\right) \xi(1-\xi) d}\left|g_{i}^{a}(k)\right\rangle .
\end{aligned}
$$

Integration over $\widetilde{\mathbf{p}}$ is done with the aid of (C.31):

$$
\begin{aligned}
\left|\psi_{g}^{1}\right\rangle= & -\mu^{2 \epsilon} \int_{\Lambda}^{e^{\delta \curlyvee} \Lambda} d k^{+} \int \frac{d^{d} \mathbf{k}}{(2 \pi)^{d}} \int_{0}^{1} d \xi \frac{g^{3} N_{f} \rho^{a}(-\mathbf{k})\left(4 \xi^{2}-4 \xi+d\right) \mathbf{k}^{i}}{2 \sqrt{\pi k^{+}} \mathbf{k}^{2} d} \\
& \times \frac{1}{(4 \pi)^{d / 2}} \frac{d}{2} \Gamma\left(-\frac{d}{2}\right)\left(\frac{1}{\xi(1-\xi) \mathbf{k}^{2}}\right)^{1-\frac{d}{2}}\left|g_{i}^{a}(k)\right\rangle .
\end{aligned}
$$

After expanding with (C.33) and taking $\epsilon \rightarrow 0$ limit, the result becomes:

$$
\begin{aligned}
\left|\psi_{g}^{1}\right\rangle= & -\int_{\Lambda}^{e^{\delta \curlyvee} \Lambda} d k^{+} \int d^{2} \mathbf{k} \int_{0}^{1} d \xi \frac{g^{3} N_{f} \rho^{a}(-\mathbf{k}) \mathbf{k}^{i}}{64 \pi^{7 / 2} \sqrt{k^{+}} \mathbf{k}^{2}} \\
& \times\left(\left(4 \xi^{2}-4 \xi+2\right)\left[-\frac{2}{\epsilon}+\ln \left(\frac{\xi(1-\xi) \mathbf{k}^{2}}{\mu^{2}}\right)+\gamma-\ln 4 \pi-1\right]+2\right)\left|g_{i}^{a}(k)\right\rangle .
\end{aligned}
$$

Finally, integrating over $\xi$ according to (C.1):

$$
\begin{aligned}
& \left|\psi_{g}^{1}\right\rangle \\
& =-\int_{\Lambda}^{e^{\delta \curlyvee} \Lambda} d k^{+} \int d^{2} \mathbf{k} \frac{g^{3} N_{f} \rho^{a}(-\mathbf{k}) \mathbf{k}^{i}}{64 \pi^{7 / 2} \sqrt{k^{+}} \mathbf{k}^{2}}\left(\frac{4}{3}\left[-\frac{2}{\epsilon}+\ln \left(\frac{\mathbf{k}^{2}}{\mu^{2}}\right)+\gamma-\ln 4 \pi\right]-\frac{20}{9}\right)\left|g_{i}^{a}(k)\right\rangle .
\end{aligned}
$$

which we can write as in (3.50).

\section{G.2 Supplement for computation of $\left|\psi_{g}^{2}\right\rangle$}

$\left|\psi_{g}^{2}\right\rangle$ is defined in (3.51). After inserting the matrix elements, (2.49) and (3.22),

$$
\begin{aligned}
\left|\psi_{g}^{2}\right\rangle= & -\int_{\Lambda}^{e^{\delta \curlyvee} \Lambda} d k^{+} \int_{\Lambda}^{k^{+}-\Lambda} d p^{+} \int d^{2} \mathbf{k} d^{2} \mathbf{p} \\
& \times \frac{g^{2} f^{a b c} f^{d b c}}{\left(\frac{\mathbf{k}^{2}}{2 k^{+}}\right)\left(\frac{\mathbf{p}^{2}}{2 p^{+}}+\frac{(\mathbf{k}-\mathbf{p})^{2}}{2\left(k^{+}-p^{+}\right)}\right)\left(\frac{\mathbf{k}^{2}}{2 k^{+}}\right) 16(2 \pi)^{3} k^{+} p^{+}\left(k^{+}-p^{+}\right)} \\
& \times\left(\left[2 \mathbf{p}^{l}-\frac{2 p^{+}}{k^{+}} \mathbf{k}^{l}\right] \delta_{j k}+\left[2 \mathbf{k}^{j}-\frac{2 k^{+}}{p^{+}} \mathbf{p}^{j}\right] \delta_{l k}+\left[\frac{k^{+}+p^{+}}{k^{+}-p^{+}}\left(\mathbf{k}^{k}-\mathbf{p}^{k}\right)-\mathbf{k}^{k}-\mathbf{p}^{k}\right] \delta_{l j}\right) \\
& \times\left(\left[2 \mathbf{p}^{i}-\frac{2 p^{+}}{k^{+}} \mathbf{k}^{i}\right] \delta_{j k}+\left[2 \mathbf{k}^{j}-\frac{2 k^{+}}{p^{+}} \mathbf{p}^{j}\right] \delta_{i k}+\left[\frac{k^{+}+p^{+}}{k^{+}-p^{+}}\left(\mathbf{k}^{k}-\mathbf{p}^{k}\right)-\mathbf{k}^{k}-\mathbf{p}^{k}\right] \delta_{i j}\right) \\
& \times\left(\frac{g \rho^{a}(-\mathbf{k}) \mathbf{k}^{i}}{4 \pi^{3 / 2}\left|k^{+}\right|^{3 / 2}}\right)\left|g_{l}^{d}(k)\right\rangle .
\end{aligned}
$$


By changing variables according to (3.34) and (3.45) we can write the last result as:

$$
\begin{aligned}
\left|\psi_{g}^{2}\right\rangle= & -\int_{\Lambda}^{e^{\delta \curlyvee} \Lambda} d k^{+} \int d^{2} \mathbf{k} d^{2} \widetilde{\mathbf{p}} \int_{\frac{\Lambda}{k^{+}}}^{1-\frac{\Lambda}{k^{+}}} d \xi \frac{g^{3} f^{a b c} f^{d b c} \rho^{a}(-\mathbf{k}) \mathbf{k}^{i}}{8(2 \pi)^{3} \pi^{3 / 2} \xi(1-\xi) \mathbf{k}^{4}\left(\mathbf{k}^{2} \xi(1-\xi)+\widetilde{\mathbf{p}}^{2}\right) \sqrt{k^{+}}} \\
& \times\left(2 \widetilde{\mathbf{p}}^{l} \delta_{j k}-\frac{2}{\xi} \widetilde{\mathbf{p}}^{j} \delta_{l k}-\frac{2}{1-\xi} \widetilde{\mathbf{p}}^{k} \delta_{l j}\right)\left(2 \widetilde{\mathbf{p}}^{i} \delta_{j k}-\frac{2}{\xi} \widetilde{\mathbf{p}}^{j} \delta_{i k}-\frac{2}{1-\xi} \widetilde{\mathbf{p}}^{k} \delta_{i j}\right)\left|g_{l}^{d}(k)\right\rangle .
\end{aligned}
$$

After some algebra:

$$
\begin{aligned}
\left|\psi_{g}^{2}\right\rangle= & -\int_{\Lambda}^{e^{\delta \curlyvee} \Lambda} d k^{+} \int d^{2} \mathbf{k} d^{2} \widetilde{\mathbf{p}} \int_{\frac{\Lambda}{k^{+}}}^{1-\frac{\Lambda}{k^{+}}} d \xi \frac{g^{3} N_{c} \rho^{a}(-\mathbf{k}) \mathbf{k}^{i} \widetilde{\mathbf{p}}^{2}}{16 \pi^{9 / 2} \mathbf{k}^{4}\left(\mathbf{k}^{2} \xi(1-\xi)+\widetilde{\mathbf{p}}^{2}\right) \sqrt{k^{+}}} \\
& \times\left(1+\frac{1}{\xi^{2}}+\frac{1}{(1-\xi)^{2}}\right)\left|g_{i}^{a}(k)\right\rangle .
\end{aligned}
$$

By replacing the measure according to (3.28):

$$
\begin{aligned}
\left|\psi_{g}^{2}\right\rangle= & -\mu^{2 \epsilon} \int_{\Lambda}^{e^{\delta \curlyvee} \Lambda} d k^{+} \int \frac{d^{d} \mathbf{k}}{(2 \pi)^{d}} \frac{d^{d} \widetilde{\mathbf{p}}}{(2 \pi)^{d}} \int_{\frac{\Lambda}{k^{+}}}^{1-\frac{\Lambda}{k^{+}}} d \xi \frac{g^{3} N_{c} \rho^{a}(-\mathbf{k}) \mathbf{k}^{i} \widetilde{\mathbf{p}}^{2}}{\sqrt{\pi} \mathbf{k}^{4}\left(\mathbf{k}^{2} \xi(1-\xi)+\widetilde{\mathbf{p}}^{2}\right) \sqrt{k^{+}}} \\
& \times\left(1+\frac{1}{\xi^{2}}+\frac{1}{(1-\xi)^{2}}\right)\left|g_{i}^{a}(k)\right\rangle .
\end{aligned}
$$

We can now compute the integration over $\mathbf{p}$ with the aid of the integral (C.31):

$$
\begin{aligned}
\left|\psi_{g}^{2}\right\rangle= & -\mu^{2 \epsilon} \int_{\Lambda}^{e^{\delta \curlyvee} \Lambda} d k^{+} \int \frac{d^{d} \mathbf{k}}{(2 \pi)^{d}} \int_{\frac{\Lambda}{k^{+}}}^{1-\frac{\Lambda}{k^{+}}} d \xi \frac{g^{3} N_{c} \rho^{a}(-\mathbf{k}) \mathbf{k}^{i}}{\sqrt{\pi} \mathbf{k}^{2} \sqrt{k^{+}}} \\
& \times\left(\frac{d}{2(4 \pi)^{d / 2}} \Gamma\left(-\frac{d}{2}\right)\left(\xi(1-\xi) \mathbf{k}^{2}\right)^{d / 2-1}\right)\left(\xi(1-\xi)-2+\frac{1}{\xi(1-\xi)}\right)\left|g_{i}^{a}(k)\right\rangle .
\end{aligned}
$$

After expanding with the aid of (C.33) taking the $\epsilon \rightarrow 0$ limit and, the last result becomes:

$$
\begin{aligned}
\left|\psi_{g}^{2}\right\rangle= & -\int_{\Lambda}^{e^{\delta \curlyvee} \Lambda} d k^{+} \int d^{2} \mathbf{k} \int_{\frac{\Lambda}{k^{+}}}^{1-\frac{\Lambda}{k^{+}}} d \xi \frac{g^{3} N_{c} \rho^{a}(-\mathbf{k}) \mathbf{k}^{i}}{16 \pi^{7 / 2} \mathbf{k}^{2} \sqrt{k^{+}}}\left(\xi(1-\xi)-2+\frac{1}{\xi(1-\xi)}\right) \\
& \times\left(-\frac{2}{\epsilon}+\ln \left(\frac{\mathbf{k}^{2} \xi(1-\xi)}{\mu_{\overline{M S}}^{2}}\right)\right)\left|g_{i}^{a}(k)\right\rangle .
\end{aligned}
$$

The relevant integral for the integration over $\xi$ is (C.10), the result after the integration is:

$$
\begin{aligned}
\left|\psi_{g \rho}^{2}\right\rangle \equiv & \left|\psi_{g}^{2}\right\rangle=\int_{\Lambda}^{e^{\delta \curlyvee} \Lambda} d k^{+} \int d^{2} \mathbf{k} \frac{g^{3} N_{c} \rho^{a}(-\mathbf{k}) \mathbf{k}^{i}}{32 \pi^{7 / 2} \mathbf{k}^{2} \sqrt{k^{+}}} \\
& \times\left(\left[\frac{11}{3}+4 \ln \left(\frac{\Lambda}{k^{+}}\right)\right]\left[-\frac{2}{\epsilon}+\ln \left(\frac{\mathbf{k}^{2}}{\mu_{\overline{M S}}^{2}}\right)\right]+2 \ln ^{2}\left(\frac{\Lambda}{k^{+}}\right)-\frac{67}{9}+\frac{2 \pi^{2}}{3}\right)\left|g_{i}^{a}(k)\right\rangle .
\end{aligned}
$$




\section{G.3 Supplement for computation of $\left|\psi_{g}^{3}\right\rangle$}

$\left|\psi_{g}^{3}\right\rangle$ is defined in (3.55). After inserting the matrix elements, (2.49) and (3.26):

$$
\begin{aligned}
\left|\psi_{g}^{3 d}\right\rangle= & \int_{\Lambda}^{e^{\delta \curlyvee} \Lambda} d k^{+} \int_{\Lambda}^{k^{+}-\Lambda} d p^{+} \int d^{2} \mathbf{k} d^{2} \mathbf{p} \frac{1}{\left(\frac{\mathbf{p}^{2}}{2 p^{+}}\right)\left(\frac{\mathbf{k}^{2}}{2 k^{+}}\right)} \\
& \times\left(\frac{i g^{2} f^{a b c}\left(p^{+}+k^{+}\right) \rho^{c}(-\mathbf{k}+\mathbf{p}) \delta_{j}^{i}}{2(2 \pi)^{3} \sqrt{k^{+} p^{+}}\left(k^{+}-p^{+}\right)^{2}}\right)\left(\frac{g \rho^{a}(-\mathbf{p}) \mathbf{p}^{i}}{4 \pi^{3 / 2}\left|p^{+}\right|^{3 / 2}}\right)\left|g_{j}^{b}(k)\right\rangle .
\end{aligned}
$$

By changing variables according to (3.34), we arrive at:

$$
\left|\psi_{g}^{3 d}\right\rangle=\int_{\Lambda}^{e^{\delta \curlyvee} \Lambda} d k^{+} \int d^{2} \mathbf{k} d^{2} \mathbf{p} \int_{\frac{\Lambda}{k^{+}}}^{1-\frac{\Lambda}{k^{+}}} d \xi \frac{i g^{3} f^{a b c}(1+\xi) \rho^{c}(-\mathbf{k}+\mathbf{p}) \rho^{a}(-\mathbf{p}) \mathbf{p}^{i}}{16 \pi^{9 / 2}(1-\xi)^{2} \xi \mathbf{k}^{2} \mathbf{p}^{2} \sqrt{k^{+}}}\left|g_{i}^{b}(k)\right\rangle .
$$

Separating the last contribution to contribution with one and two $\rho$ operators according to (3.27), we notice that the contribution with one $\rho$ operator vanishes after integraion over $\mathbf{p}$. The two $\rho$ part reads:

$$
\left|\psi_{g}^{3 d}\right\rangle=\int_{\Lambda}^{e^{\delta \curlyvee} \Lambda} d k^{+} \int d^{2} \mathbf{k} d^{2} \mathbf{p} \int_{\frac{\Lambda}{k^{+}}}^{1-\frac{\Lambda}{k^{+}}} d \xi \frac{i g^{3} f^{a b c}(1+\xi) \mathbf{p}^{i}\left\{\rho^{c}(-\mathbf{k}+\mathbf{p}), \rho^{a}(-\mathbf{p})\right\}}{32 \pi^{9 / 2}(1-\xi)^{2} \xi \mathbf{k}^{2} \mathbf{p}^{2} \sqrt{k^{+}}}\left|g_{i}^{b}(k)\right\rangle .
$$

The relevant integral for the integration over $\xi$ is (C.7), the result after the integration appears in (3.56).

\section{G.4 Supplement for computation of $\left|\psi_{g}^{4}\right\rangle$ and $\left|\psi_{g}^{5}\right\rangle$}

$\left|\psi_{g}^{4}\right\rangle$ is defined in (3.60). After inserting the matrix elements, (3.25) and (3.23),

$$
\begin{aligned}
\left|\psi_{g}^{4}\right\rangle= & \frac{1}{2} \int_{\Lambda}^{e^{\delta \curlyvee} \Lambda} d k^{+} \int_{\Lambda}^{k^{+}-\Lambda} d p^{+} d q^{+} \int d^{2} \mathbf{k} d^{2} \mathbf{p} d^{2} \mathbf{q} \frac{1}{\left(\frac{\mathbf{q}^{2}}{2 q^{+}}+\frac{(\mathbf{k}-\mathbf{q})^{2}}{2\left(k^{+}-q^{+}\right)}\right)\left(\frac{\mathbf{p}^{2}}{2 p^{+}}\right)} \\
& \times\left(\delta^{d c} \delta_{i l} \delta^{(3)}(k-p-q) \frac{g \mathbf{q}^{j} \rho^{b}(\mathbf{q})}{4 \pi^{3 / 2}\left|q^{+}\right|^{3 / 2}}+\delta^{d b} \delta_{l j} \delta^{(3)}(q-p) \frac{g\left(\mathbf{k}^{i}-\mathbf{q}^{i}\right) \rho^{c}(\mathbf{k}-\mathbf{q})}{4 \pi^{3 / 2}\left|k^{+}-q^{+}\right|^{3 / 2}}\right) \\
& \times\left(\frac{i g^{2} f^{a b c}\left(k^{+}-2 q^{+}\right) \delta_{i j} \rho^{a}(-\mathbf{k})}{2(2 \pi)^{3} \sqrt{\left(k^{+}-q^{+}\right) q^{+}}\left(k^{+}\right)^{2}}\right)\left|g_{l}^{d}(p)\right\rangle .
\end{aligned}
$$

After integration over $q$ :

$$
\begin{aligned}
\left|\psi_{g}^{4}\right\rangle= & -\int_{\Lambda}^{e^{\delta \curlyvee} \Lambda} d k^{+} \int_{\Lambda}^{k^{+}-\Lambda} d p^{+} \int d^{2} \mathbf{k} d^{2} \mathbf{p} \\
& \times \frac{i g^{3} f^{a b c}\left(2 p^{+}-k^{+}\right) \rho^{c}(\mathbf{k}-\mathbf{p}) \rho^{a}(-\mathbf{k})\left(\mathbf{k}^{i}-\mathbf{p}^{i}\right)}{64 \pi^{9 / 2}\left(\frac{\mathbf{p}^{2}}{2 p^{+}}+\frac{(\mathbf{k}-\mathbf{p})^{2}}{2\left(k^{+}-p^{+}\right)}\right)\left(\frac{\mathbf{p}^{2}}{2 p^{+}}\right)\left(k^{+}\left(k^{+}-p^{+}\right)\right)^{2} \sqrt{p^{+}}}\left|g_{i}^{b}(p)\right\rangle .
\end{aligned}
$$


After changing variables according to (3.34):

$$
\begin{aligned}
\left|\psi_{g}^{4}\right\rangle= & -\int_{\Lambda}^{e^{\delta \curlyvee} \Lambda} d k^{+} \int d^{2} \mathbf{k} d^{2} \mathbf{p} \int_{\frac{\Lambda}{k^{+}}}^{1-\frac{\Lambda}{k^{+}}} d \xi \\
& \times \frac{i g^{3} f^{a b c} \rho^{c}(\mathbf{k}-\mathbf{p}) \rho^{a}(-\mathbf{k})\left(\mathbf{k}^{i}-\mathbf{p}^{i}\right)(2 \xi-1) \xi^{3 / 2}}{16 \pi^{9 / 2}\left(\xi(\mathbf{k}-\mathbf{p})^{2}+(1-\xi) \mathbf{p}^{2}\right) \mathbf{p}^{2}(1-\xi) \sqrt{k^{+}}}\left|g_{i}^{b}\left(\xi k^{+}, \mathbf{p}\right)\right\rangle .
\end{aligned}
$$

Now let us work out the case of $\left|\psi_{g}^{5}\right\rangle$ as defined in (3.61). By inserting the relevant matrix elements, (2.49), (3.23) and (3.22),

$$
\begin{aligned}
\left|\psi_{g}^{5}\right\rangle= & -\int_{\Lambda}^{e^{\delta \curlyvee} \Lambda} d k^{+} d p^{+} \int_{\Lambda}^{k^{+}-\Lambda} d q^{+} \int d^{2} \mathbf{k} d^{2} \mathbf{p} d^{2} \mathbf{q} \frac{1}{\left(\frac{\mathbf{p}^{2}}{2 p^{+}}\right)\left(\frac{(\mathbf{k}-\mathbf{q})^{2}}{2\left(k^{+}-q^{+}\right)}+\frac{\mathbf{q}^{2}}{2 q^{+}}\right)\left(\frac{\mathbf{k}^{2}}{2 k^{+}}\right)} \\
& \times\left(\delta^{d c} \delta^{k m} \delta^{(3)}(k-p-q) \frac{g \mathbf{q}^{j} \rho^{b}(\mathbf{q})}{4 \pi^{3 / 2}\left|q^{+}\right|^{3 / 2}}+\delta^{d b} \delta^{j m} \delta^{(3)}(p-q) \frac{g\left(\mathbf{k}^{k}-\mathbf{q}^{k}\right) \rho^{c}(\mathbf{k}-\mathbf{q})}{4 \pi^{3 / 2}\left|k^{+}-q^{+}\right|^{3 / 2}}\right) \\
& \times\left(\left[2 \mathbf{q}^{i}-\frac{2 q^{+}}{k^{+}} \mathbf{k}^{i}\right] \delta_{j k}+\left[2 \mathbf{k}^{j}-\frac{2 k^{+}}{q^{+}} \mathbf{q}^{j}\right] \delta_{i k}+\left[\frac{k^{+}+q^{+}}{k^{+}-q^{+}}\left(\mathbf{k}^{k}-\mathbf{q}^{k}\right)-\mathbf{k}^{k}-\mathbf{q}^{k}\right] \delta_{i j}\right) \\
& \times \frac{i g f^{a b c}}{16 \pi^{3 / 2} \sqrt{k^{+} q^{+}\left(k^{+}-q^{+}\right)}}\left(\frac{g \mathbf{k}^{i} \rho^{a}(-\mathbf{k})}{4 \pi^{3 / 2}\left|k^{+}\right| 3 / 2}\right)\left|g_{m}^{d}(p)\right\rangle .
\end{aligned}
$$

After performing the multiplications, and changing variables according to (3.34), we arrive at:

$$
\begin{aligned}
\left|\psi_{g}^{5}\right\rangle= & \int_{\Lambda}^{e^{\delta \curlyvee} \Lambda} d k^{+} \int d^{2} \mathbf{k} d^{2} \mathbf{p} \int_{\frac{\Lambda}{k^{+}}}^{1-\frac{\Lambda}{k^{+}}} d \xi \frac{i g^{3} f^{a b c} \rho^{c}(\mathbf{k}-\mathbf{p}) \rho^{a}(-\mathbf{k}) \xi^{3 / 2}}{16 \pi^{9 / 2} \mathbf{k}^{2} \mathbf{p}^{2}\left(\xi(\mathbf{k}-\mathbf{p})^{2}+(1-\xi) \mathbf{p}^{2}\right)(1-\xi) \sqrt{k^{+}}} \\
& \times\left(\left[-2 \mathbf{k} \cdot \mathbf{p}+2 \xi \mathbf{k}^{2}\right]\left(\mathbf{k}^{j}-\mathbf{p}^{j}\right)+\left[-2 \mathbf{k}^{j}+\frac{2}{\xi} \mathbf{p}^{j}\right] \mathbf{k} \cdot(\mathbf{k}-\mathbf{p})\right. \\
& \left.+\left[\mathbf{k}^{2}-\mathbf{p}^{2}-\frac{1+\xi}{1-\xi}(\mathbf{k}-\mathbf{p})^{2}\right] \mathbf{k}^{j}\right)\left|g_{j}^{b}\left(\xi k^{+}, \mathbf{p}\right)\right\rangle .
\end{aligned}
$$

Equivalently, we can write the last result as:

$$
\begin{aligned}
& \left|\psi_{g}^{5}\right\rangle=\int_{\Lambda}^{e^{\delta \curlyvee} \Lambda} d k^{+} \int d^{2} \mathbf{k} d^{2} \mathbf{p} \int_{\frac{\Lambda}{k^{+}}}^{1-\frac{\Lambda}{k^{+}}} d \xi \frac{i g^{3} f^{a b c} \rho^{c}(\mathbf{k}-\mathbf{p}) \rho^{a}(-\mathbf{k}) \xi^{3 / 2}}{16 \pi^{9 / 2} \mathbf{k}^{2} \mathbf{p}^{2}\left(\xi(\mathbf{k}-\mathbf{p})^{2}+(1-\xi) \mathbf{p}^{2}\right)(1-\xi) \sqrt{k^{+}}} \\
& \times\left(\left[(2 \xi-1) \mathbf{k}^{2}-\mathbf{p}^{2}-\frac{1+\xi}{1-\xi}(\mathbf{k}-\mathbf{p})^{2}\right] \mathbf{k}^{j}+\left[\frac{\xi-1}{\xi} \mathbf{k} \cdot \mathbf{p}+\frac{1-\xi^{2}}{\xi} \mathbf{k}^{2}\right] 2 \mathbf{p}^{j}\right)\left|g_{j}^{b}\left(\xi k^{+}, \mathbf{p}\right)\right\rangle .
\end{aligned}
$$

By adding together $\left|\psi_{g}^{4}\right\rangle$ and $\left|\psi_{g}^{5}\right\rangle$, we arrive at:

$$
\begin{aligned}
\left|\psi_{g}^{4+5}\right\rangle \equiv & \left|\psi_{g}^{4}\right\rangle+\left|\psi_{g}^{5}\right\rangle \\
= & \int_{\Lambda}^{e^{\delta \curlyvee} \Lambda} d k^{+} \int d^{2} \mathbf{k} d^{2} \mathbf{p} \int_{\frac{\Lambda}{k^{+}}}^{1-\frac{\Lambda}{k^{+}}} d \xi \frac{i g^{3} f^{a b c} \rho^{c}(\mathbf{k}-\mathbf{p}) \rho^{a}(-\mathbf{k}) \xi^{3 / 2}}{16 \pi^{9 / 2} \mathbf{k}^{2} \mathbf{p}^{2}\left(\xi(\mathbf{k}-\mathbf{p})^{2}+(1-\xi) \mathbf{p}^{2}\right)(1-\xi) \sqrt{k^{+}}} \\
& \times\left(\left[\frac{2}{\xi}\left(\mathbf{k}^{2}-\mathbf{k} \cdot \mathbf{p}\right)-\mathbf{k}^{2}+2 \mathbf{k} \cdot \mathbf{p}\right] \mathbf{p}^{j}-\left[\mathbf{p}^{2}+\frac{1+\xi}{1-\xi}(\mathbf{k}-\mathbf{p})^{2}\right] \mathbf{k}^{j}\right)\left|g_{j}^{b}\left(\xi k^{+}, \mathbf{p}\right)\right\rangle .
\end{aligned}
$$


from (G.26) we can directly deduce the part which is proportional to two $\rho$ operators. Below, we will focus on the part which involve one $\rho$ operator only, which can be isolated via the prescription (3.27).

- One $\rho$ part. Let us now focus on the contribution which involves only one $\rho$ operator:

$$
\begin{aligned}
& \left|\psi_{g}^{4+5}\right\rangle \\
& =-\int_{\Lambda}^{e^{\delta \curlyvee} \Lambda} d k^{+} \int d^{2} \mathbf{k} d^{2} \mathbf{p} \int_{\frac{\Lambda}{k^{+}}}^{1-\frac{\Lambda}{k^{+}}} d \xi \frac{g^{3} N_{c} \rho^{a}(-\mathbf{p}) \xi^{3 / 2}}{32 \pi^{9 / 2} \mathbf{k}^{2} \mathbf{p}^{2}\left(\xi(\mathbf{k}-\mathbf{p})^{2}+(1-\xi) \mathbf{p}^{2}\right)(1-\xi) \sqrt{k^{+}}} \\
& \quad \times\left(\left[\frac{2}{\xi}\left(\mathbf{k}^{2}-\mathbf{k} \cdot \mathbf{p}\right)-\mathbf{k}^{2}+2 \mathbf{k} \cdot \mathbf{p}\right] \mathbf{p}^{j}-\left[\mathbf{p}^{2}+\frac{1+\xi}{1-\xi}(\mathbf{k}-\mathbf{p})^{2}\right] \mathbf{k}^{j}\right)\left|g_{j}^{a}\left(\xi k^{+}, \mathbf{p}\right)\right\rangle .
\end{aligned}
$$

We rewrite the denominator of $\left|\psi_{g}^{4+5}\right\rangle$ by using Feynman parameter. We introduce the following definitions:

$$
\alpha \equiv 1-x+x \xi, \quad \Delta \equiv \frac{x\left(1-x+x \xi-x \xi^{2}\right)}{\alpha^{2}} .
$$

and perform the shift $\mathbf{k} \longrightarrow \mathbf{k}+\frac{x \xi}{\alpha} \mathbf{p}$. Then we arrive at:

$$
\begin{aligned}
\left|\psi_{g}^{4+5}\right\rangle & \\
= & -\int_{\Lambda}^{e^{\delta \curlyvee} \Lambda} d k^{+} \int d^{2} \mathbf{k} d^{2} \mathbf{p} \int_{\frac{\Lambda}{k^{+}}}^{1-\frac{\Lambda}{k^{+}}} d \xi \int_{0}^{1} d x \frac{g^{3} N_{c} \rho^{a}(-\mathbf{p}) \xi^{3 / 2}}{32 \pi^{9 / 2} \sqrt{k^{+}}(1-\xi) \mathbf{p}^{2}\left(\mathbf{k}^{2}+\Delta \mathbf{p}^{2}\right)^{2}} \\
& \times\left(\left[\frac{2}{\xi}\left(\left(\mathbf{k}+\frac{x \xi}{\alpha} \mathbf{p}\right)^{2}-\left(\mathbf{k}+\frac{x \xi}{\alpha} \mathbf{p}\right) \cdot \mathbf{p}\right)-\left(\mathbf{k}+\frac{x \xi}{\alpha} \mathbf{p}\right)^{2}+2\left(\mathbf{k}+\frac{x \xi}{\alpha} \mathbf{p}\right) \cdot \mathbf{p}\right] \mathbf{p}^{j}\right. \\
& \left.-\left[\mathbf{p}^{2}+\frac{1+\xi}{1-\xi}\left(\mathbf{k}+\left(\frac{x \xi}{\alpha}-1\right) \mathbf{p}\right)^{2}\right]\left(\mathbf{k}^{j}+\frac{x \xi}{\alpha} \mathbf{p}^{j}\right)\right)\left|g_{j}^{a}\left(\xi k^{+}, \mathbf{p}\right)\right\rangle .
\end{aligned}
$$

After changing the measure according to (3.28), and using the relation (3.29):

$$
\begin{aligned}
\left|\psi_{g \rho}^{4+5}\right\rangle= & -\mu^{2 \epsilon} \int_{\Lambda}^{e^{\delta \Upsilon} \Lambda} d k^{+} \int \frac{d^{d} \mathbf{k}}{(2 \pi)^{d}} \frac{d^{d} \widetilde{\mathbf{p}}}{(2 \pi)^{d}} \int_{\frac{\Lambda}{k^{+}}}^{1-\frac{\Lambda}{k^{+}}} d \xi \int_{0}^{1} d x \\
& \times \frac{g^{3} N_{c} \rho^{a}(-\mathbf{p}) \xi^{3 / 2} \mathbf{p}^{j}}{2 \sqrt{\pi k^{+}}(1-\xi) \alpha^{2} \mathbf{p}^{2}\left(\mathbf{k}^{2}+\Delta \mathbf{p}^{2}\right)^{2}}\left(\left[\left(\frac{2}{\xi}-1+\frac{2}{d} \frac{1+\xi}{1-\xi} \frac{1-x}{\alpha}-\frac{x \xi}{\alpha} \frac{1+\xi}{1-\xi}\right) \mathbf{k}^{2}\right.\right. \\
& \left.+\left(1-\frac{2}{\xi}-\frac{(1+\xi)(1-x)}{\alpha(1-\xi)}\right) \frac{x(1-x) \xi}{\alpha^{2}} \mathbf{p}^{2}\right] \mathbf{p}^{j}-\left[\left(1+\frac{1+\xi}{1-\xi} \frac{x-1}{\alpha}\right.\right. \\
& \left.\left.\left.-\frac{2}{d}\left(\frac{2-\xi}{\xi} \frac{x \xi}{\alpha}-\frac{x \xi}{\alpha} \frac{1+\xi}{1-\xi} \frac{x-1}{\alpha}-\frac{1-\xi}{\xi}\right)\right) \mathbf{p}^{2}+\frac{1+\xi}{1-\xi} \mathbf{k}^{2}\right] \mathbf{k}^{j}\right)\left|g_{j}^{a}\left(\xi k^{+}, \mathbf{p}\right)\right\rangle .
\end{aligned}
$$

After integration over $\mathbf{k}$ with the aid of (C.31) and (C.32):

$$
\left|\psi_{g \rho}^{4+5}\right\rangle=-\mu^{2 \epsilon} \int_{\Lambda}^{e^{\delta \curlyvee} \Lambda} d k^{+} \int \frac{d^{d} \mathbf{p}}{(2 \pi)^{d}} \int_{\frac{\Lambda}{k^{+}}}^{1-\frac{\Lambda}{k^{+}}} d \xi \int_{0}^{1} d x \frac{g^{3} N_{c} \rho^{a}(-\mathbf{p}) \xi^{3 / 2} \mathbf{p}^{j}}{2 \sqrt{\pi}(1-\xi) \alpha^{2} \mathbf{p}^{2} \sqrt{k^{+}}}
$$




$$
\begin{aligned}
& \times\left(\left[1-\frac{2}{\xi}-\frac{1+\xi}{1-\xi} \frac{1-x}{\alpha}\right] \frac{x(1-x) \xi}{\alpha^{2}} \mathbf{p}^{2} \frac{1}{(4 \pi)^{d / 2}} \Gamma\left(2-\frac{d}{2}\right)\left(\frac{1}{\Delta}\right)^{2-\frac{d}{2}}\right. \\
& \left.+\left[\frac{2}{\xi}-1+\frac{2}{d} \frac{1+\xi}{1-\xi} \frac{1-x}{\alpha}-\frac{x \xi}{\alpha} \frac{1+\xi}{1-\xi}\right] \frac{1}{(4 \pi)^{d / 2}} \frac{d}{2} \Gamma\left(1-\frac{d}{2}\right)\left(\frac{1}{\Delta}\right)^{1-\frac{d}{2}}\right)\left|g_{j}^{a}\left(\xi k^{+}, \mathbf{p}\right)\right\rangle
\end{aligned}
$$

After taking the $\epsilon \rightarrow 0$ limit and expanding with the aid of (C.33), the last result becomes:

$$
\begin{aligned}
& \left|\psi_{g \rho}^{4+5}\right\rangle=-\int_{\Lambda}^{e^{\delta \curlyvee} \Lambda} d k^{+} \int d^{2} \mathbf{p} \int_{\frac{\Lambda}{k^{+}}}^{1-\frac{\Lambda}{k^{+}}} d \xi \int_{0}^{1} d x \frac{g^{3} N_{c} \rho^{a}(-\mathbf{p}) \xi^{3 / 2} \mathbf{p}^{j}}{32 \pi^{7 / 2}(1-\xi) \alpha^{2} \mathbf{p}^{2} \sqrt{k^{+}}} \\
& \quad \times\left(\left[1-\frac{2}{\xi}-\frac{1+\xi}{1-\xi} \frac{1-x}{\alpha}\right] \frac{(1-x) \xi}{1-x+x \xi-x \xi^{2}}-\frac{2}{\xi}+1+\frac{x \xi}{\alpha} \frac{1+\xi}{1-\xi}\right. \\
& \left.\quad-\left[\frac{2}{\xi}-1+\frac{1+\xi}{1-\xi} \frac{1-x-x \xi}{\alpha}\right]\left[-\frac{2}{\epsilon}+\ln \left(\frac{x\left(1-x+x \xi-x \xi^{2}\right) \mathbf{p}^{2}}{\alpha^{2} \mu_{\overline{M S}}^{2}}\right)\right]\right)\left|g_{j}^{a}\left(\xi k^{+}, \mathbf{p}\right)\right\rangle .
\end{aligned}
$$

The integration over $x$ can be performed using the following integral:

$$
\begin{aligned}
& \int_{0}^{1} d x \frac{1}{(1-x+x \xi)^{2}}\left(\left[1-\frac{2}{\xi}-\frac{1+\xi}{1-\xi} \frac{1-x}{1-x+x \xi}\right] \frac{(1-x) \xi}{1-x+x \xi-x \xi^{2}}-\frac{2}{\xi}+1\right. \\
& \left.+\frac{x \xi}{1-x+x \xi} \frac{1+\xi}{1-\xi}-\left[\frac{2}{\xi}-1+\frac{1+\xi}{1-\xi} \frac{1-x-x \xi}{1-x+x \xi}\right]\left[-\frac{2}{\epsilon}+\ln \left(\frac{x\left(1-x+x \xi-x \xi^{2}\right) \mathbf{p}^{2}}{(1-x+x \xi)^{2} \mu \frac{2}{M S}}\right)\right]\right) \\
& =-\frac{1}{\xi^{2}}\left((\xi-2) \ln (\xi)+(\xi+1) \ln (1-\xi)-(\xi-2)\left[-\frac{2}{\epsilon}+\ln \left(\frac{\mathbf{p}^{2}}{\mu_{\overline{M S}}^{2}}\right)\right]\right) .
\end{aligned}
$$

The final result is quoted in (3.64) after renaming $\mathbf{p} \leftrightarrow \mathbf{k}$.

\section{G.5 Supplement for computation of $\left|\psi_{g}^{\mathbf{6}}\right\rangle$}

$\left|\psi_{g}^{6}\right\rangle$ is defined in (3.67). By inserting the relevant matrix elements, (2.49), (3.23) and (3.22), we arrive at:

$$
\begin{aligned}
& \left|\psi_{g}^{6}\right\rangle=\int_{\Lambda}^{e^{\delta \curlyvee} \Lambda} d k^{+} \int_{\frac{\Lambda}{k^{+}}}^{1-\frac{\Lambda}{k^{+}}} d p^{+} d q^{+} \int d^{2} \mathbf{k} d^{2} \mathbf{p} d^{2} \mathbf{q} \\
& \times \frac{i g f^{b c d}}{\left(\frac{\mathbf{q}^{2}}{2 q^{+}}\right)\left(\frac{\mathbf{p}^{2}}{2 p^{+}}+\frac{(\mathbf{k}-\mathbf{p})^{2}}{2\left(k^{+}-p^{+}\right)}\right)\left(\frac{\mathbf{k}^{2}}{2 k^{+}}\right) 16 \pi^{3 / 2} \sqrt{k^{+} p^{+}\left(k^{+}-p^{+}\right)}} \\
& \times\left[\left(2 \mathbf{p}^{l}-\frac{2 p^{+}}{k^{+}} \mathbf{k}^{l}\right) \delta_{n j}+\left(2 \mathbf{k}^{j}-\frac{2 k^{+}}{p^{+}} \mathbf{p}^{j}\right) \delta_{l n}+\left(\frac{k^{+}+p^{+}}{k^{+}-p^{+}}\left(\mathbf{k}^{n}-\mathbf{p}^{n}\right)-\mathbf{k}^{n}-\mathbf{p}^{n}\right) \delta_{l j}\right] \\
& \times\left(\delta^{a c} \delta^{n i} \delta^{(3)}(q-k+p) \frac{g \mathbf{p}^{j} \rho^{b}(-\mathbf{p})}{4 \pi^{3 / 2}\left|p^{+}\right|^{3 / 2}}+\delta^{a b} \delta^{n i} \delta^{(3)}(q-p) \frac{g\left(\mathbf{k}^{n}-\mathbf{p}^{n}\right) \rho^{c}(-\mathbf{k}+\mathbf{p})}{4 \pi^{3 / 2}\left|k^{+}-p^{+}\right|^{3 / 2}}\right) \\
& \times\left(\frac{g \rho^{a}(-\mathbf{q}) \mathbf{q}^{i}}{4 \pi^{3 / 2}\left|q^{+}\right|^{3 / 2}}\right)\left|g_{l}^{d}(k)\right\rangle .
\end{aligned}
$$

After simplifications the last result becomes:

$$
\left|\psi_{g}^{6}\right\rangle=\int_{\Lambda}^{e^{\delta \curlyvee} \Lambda} d k^{+} \int d^{2} \mathbf{k} d^{2} \mathbf{p} \int_{\frac{\Lambda}{k^{+}}}^{1-\frac{\Lambda}{k^{+}}} d \xi \frac{i g^{3} f^{\mathrm{bad}} \rho^{b}(-\mathbf{p}) \rho^{a}(-\mathbf{k}+\mathbf{p})}{16 \pi^{9 / 2} \xi(\mathbf{k}-\mathbf{p})^{2}\left((1-\xi) \mathbf{p}^{2}+\xi(\mathbf{k}-\mathbf{p})^{2}\right) \mathbf{k}^{2} \sqrt{k^{+}}}
$$




$$
\begin{aligned}
& \times\left[\left(2 \mathbf{p}^{l}-2 \xi \mathbf{k}^{l}\right) \mathbf{p} \cdot(\mathbf{k}-\mathbf{p})+\left(2 \mathbf{k} \cdot \mathbf{p}-\frac{2}{\xi} \mathbf{p}^{2}\right)(\mathbf{k}-\mathbf{p})^{l}\right. \\
& \left.+\left(\frac{1+\xi}{1-\xi}(\mathbf{k}-\mathbf{p})^{2}-\mathbf{k}^{2}+\mathbf{p}^{2}\right) \mathbf{p}^{l}\right]\left|g_{l}^{d}(k)\right\rangle .
\end{aligned}
$$

Which we can equivalently write as in (3.68). We can now isolate the contribution to one and two $\rho$ via the prescription in (3.27).

- One $\rho$ part. Let us now focus on the contribution which involves only one $\rho$ operator:

$$
\begin{aligned}
& \left|\psi_{g \rho \rho}^{6}\right\rangle \\
& =\int_{\Lambda}^{e^{\delta \curlyvee} \Lambda} d k^{+} \int d^{2} \mathbf{k} d^{2} \mathbf{p} \int_{\frac{\Lambda}{k^{+}}}^{1-\frac{\Lambda}{k^{+}}} d \xi \int_{0}^{1} d x \frac{g^{3} f^{\mathrm{bad}} f^{b a c} \rho^{c}(-\mathbf{k})}{32 \pi^{9 / 2} \xi\left((\mathbf{p}-\alpha \mathbf{k})^{2}+\alpha x(1-\xi) \mathbf{k}^{2}\right)^{2} \mathbf{k}^{2} \sqrt{k^{+}}} \\
& \times\left[\left(-2(1-\xi) \mathbf{k} \cdot \mathbf{p}+\frac{2\left(1-\xi^{2}\right)}{\xi} \mathbf{p}^{2}\right) \mathbf{k}^{l}+\left(\mathbf{k}^{2}-\frac{1+\xi}{1-\xi}(\mathbf{k}-\mathbf{p})^{2}+\frac{\xi-2}{\xi} \mathbf{p}^{2}\right) \mathbf{p}^{l}\right]\left|g_{l}^{d}(k)\right\rangle .
\end{aligned}
$$

The denominators of the last expression can be rewritten by using Feynman parameter. We introduce again the variable $\alpha$ which was defined in (G.28). After shifting the momenta according to $\mathbf{p} \longrightarrow \mathbf{p}+\alpha \mathbf{k}$ we arrive at:

$$
\begin{aligned}
& \left|\psi_{g \rho}^{6}\right\rangle=\int_{\Lambda}^{e^{\delta \curlyvee} \Lambda} d k^{+} \int d^{2} \mathbf{k} d^{2} \mathbf{p} \int_{\frac{\Lambda}{k^{+}}}^{1-\frac{\Lambda}{k^{+}}} d \xi \int_{0}^{1} d x \frac{g^{3} N_{c} \rho^{a}(-\mathbf{k})}{32 \pi^{9 / 2} \xi\left(\mathbf{p}^{2}+\alpha x(1-\xi) \mathbf{k}^{2}\right)^{2} \mathbf{k}^{2} \sqrt{k^{+}}} \\
& \times\left(\left(-2(1-\xi) \mathbf{k} \cdot(\mathbf{p}+\alpha \mathbf{k})+\frac{2\left(1-\xi^{2}\right)}{\xi}(\mathbf{p}+\alpha \mathbf{k})^{2}\right) \mathbf{k}^{l}\right. \\
& \left.+\left(\mathbf{k}^{2}-\frac{1+\xi}{1-\xi}((1-\alpha) \mathbf{k}-\mathbf{p})^{2}+\frac{\xi-2}{\xi}(\mathbf{p}+\alpha \mathbf{k})^{2}\right)\left(\mathbf{p}^{l}+\alpha \mathbf{k}^{l}\right)\right)\left|g_{l}^{a}(k)\right\rangle .
\end{aligned}
$$

Changing the measure according to (3.28) and using the relation (3.29) we can rewrite the last result as:

$$
\begin{aligned}
& \left|\psi_{g \rho}^{6}\right\rangle=\mu^{2 \epsilon} \int_{\Lambda}^{e^{\delta \curlyvee} \Lambda} d k^{+} \int \frac{d^{d} \mathbf{k}}{(2 \pi)^{d}} \frac{d^{d} \mathbf{p}}{(2 \pi)^{d}} \int_{\frac{\Lambda}{k^{+}}}^{1-\frac{\Lambda}{k^{+}}} d \xi \int_{0}^{1} d x \frac{g^{3} N_{c} \rho^{a}(-\mathbf{k})}{2 \sqrt{\pi k^{+}} \xi\left(\mathbf{p}^{2}+\alpha x(1-\xi) \mathbf{k}^{2}\right)^{2} \mathbf{k}^{2}} \\
& \times\left(\left[1-\frac{(1-\alpha)^{2}(1+\xi)}{(1-\xi)}+\frac{2 \alpha(1-\alpha)(1+\xi)}{(1-\xi) d}+\frac{(2+d) \alpha^{2}(\xi-2)+4 \alpha\left(1-\xi^{2}\right)}{\xi d}\right.\right. \\
& \left.-\frac{2 \xi(1-\xi)}{\xi d}\right] \mathbf{k}^{2} \mathbf{p}^{l}+\left[\frac{\alpha(\xi-2)(d+2)+2 d\left(1-\xi^{2}\right)}{\xi d}+\frac{2(1-\alpha)(1+\xi)-\alpha(1+\xi) d}{(1-\xi) d}\right] \mathbf{p}^{2} \mathbf{k}^{l} \\
& +\left[\frac{\alpha^{3}(\xi-2)+2 \alpha^{2}\left(1-\xi^{2}\right)}{\xi}-\frac{\alpha(1-\alpha)^{2}(1+\xi)}{1-\xi}+\alpha(2 \xi-1)\right] \mathbf{k}^{2} \mathbf{k}^{l} \\
& \left.+\left[\frac{\xi-2}{\xi}-\frac{1+\xi}{1-\xi}\right] \mathbf{p}^{2} \mathbf{p}^{l}\right)\left|g_{l}^{a}(k)\right\rangle .
\end{aligned}
$$

After integration over $\mathbf{p}$ by using (C.31) and (C.32):

$$
\left|\psi_{g \rho}^{6}\right\rangle=\mu^{2 \epsilon} \int_{\Lambda}^{e^{\delta \curlyvee} \Lambda} d k^{+} \int \frac{d^{d} \mathbf{k}}{(2 \pi)^{d}} \int_{\frac{\Lambda}{k^{+}}}^{1-\frac{\Lambda}{k^{+}}} d \xi \int_{0}^{1} d x \frac{g^{3} N_{c} \rho^{a}(-\mathbf{k}) \mathbf{k}^{l}}{2 \sqrt{\pi k^{+}} \xi \mathbf{k}^{2}}\left(\frac{\Gamma\left(2-\frac{d}{2}\right)}{(4 \pi)^{d / 2}}\right.
$$




$$
\begin{aligned}
& \times\left(\frac{1}{\alpha x(1-\xi) \mathbf{k}^{2}}\right)^{2-\frac{d}{2}}\left[\frac{\alpha^{3}(\xi-2)+2 \alpha^{2}\left(1-\xi^{2}\right)}{\xi}-\frac{\alpha(1-\alpha)^{2}(1+\xi)}{1-\xi}+(2 \xi-1) \alpha\right] \mathbf{k}^{2} \\
& \left.+\frac{\Gamma\left(1-\frac{d}{2}\right)}{2(4 \pi)^{d / 2}}\left(\frac{1}{\alpha x(1-\xi) \mathbf{k}^{2}}\right)^{1-\frac{d}{2}}\left[\frac{2\left(1-\xi^{2}\right) d+\alpha(\xi-2) d+2 \alpha(\xi-2)}{2 \xi}\right]\right)\left|g_{l}^{a}(k)\right\rangle . \\
& +\frac{2(1-\alpha)(1+\xi)-\alpha(1+\xi) d}{2(1-\xi)}
\end{aligned}
$$

After taking the $\epsilon \rightarrow 0$ limit and expanding with the aid of (C.33), the last result becomes:

$$
\begin{aligned}
& \left|\psi_{g \rho}^{6}\right\rangle=\int_{\Lambda}^{e^{\delta \curlyvee} \Lambda} d k^{+} \int d^{2} \mathbf{k} \int_{\frac{\Lambda}{k^{+}}}^{1-\frac{\Lambda}{k^{+}}} d \xi \int_{0}^{1} d x \frac{g^{3} N_{c} \rho^{a}(-\mathbf{k}) \mathbf{k}^{l}}{32 \pi^{7 / 2} \xi \mathbf{k}^{2} \sqrt{k^{+}}}\left(\frac{2\left(1-\xi^{2}\right)+\alpha(\xi-2)}{\xi}\right) \\
& -\frac{\alpha(1+\xi)}{1-\xi}+\left[\frac{\alpha^{3}(\xi-2)+2 \alpha^{2}\left(1-\xi^{2}\right)}{\xi}-\frac{\alpha(1-\alpha)^{2}(1+\xi)}{1-\xi}+(2 \xi-1) \alpha\right] \frac{1}{\alpha x(1-\xi)} \\
& \quad-\left[\frac{2\left(1-\xi^{2}\right)+2 \alpha(\xi-2)}{\xi}+\frac{(1-\alpha)(1+\xi)-\alpha(1+\xi)}{1-\xi}\right] \\
& \left.\quad \times\left[-\frac{2}{\epsilon}+\ln \left(\frac{\alpha x(1-\xi) \mathbf{k}^{2}}{4 \pi e^{-\gamma} \mu^{2}}\right)\right]\right)\left|g_{l}^{a}(k)\right\rangle .
\end{aligned}
$$

After integration over $x$ :

$$
\begin{aligned}
\left|\psi_{g \rho}^{6}\right\rangle= & -\int_{\Lambda}^{e^{\delta \curlyvee} \Lambda} d k^{+} \int d^{2} \mathbf{k} \int_{\frac{\Lambda}{k^{+}}}^{1-\frac{\Lambda}{k^{+}}} d \xi \frac{g^{3} N_{c} \rho^{a}(-\mathbf{k}) \mathbf{k}^{l}}{32 \pi^{7 / 2} \xi(1-\xi) \mathbf{k}^{2} \sqrt{k^{+}}} \\
& \times\left((\xi-2) \ln (\xi)-(1+\xi) \ln (1-\xi)-(1+\xi)\left[-\frac{2}{\epsilon}+\ln \left(\frac{\mathbf{k}^{2}}{\mu_{\overline{M S}}^{2}}\right)\right]\right)\left|g_{l}^{a}(k)\right\rangle .
\end{aligned}
$$

The result which is obtained after integration over $\xi$, using equations (C.12) and (C.13), is:

$$
\begin{aligned}
\left|\psi_{g \rho}^{6}\right\rangle \equiv & -\int_{\Lambda}^{e^{\delta \curlyvee} \Lambda} d k^{+} \int d^{2} \mathbf{k} \frac{g^{3} N_{c} \rho^{a}(-\mathbf{k}) \mathbf{k}^{i}}{32 \pi^{7 / 2} \mathbf{k}^{2} \sqrt{k^{+}}} \\
& \times\left(3 \ln \left(\frac{\Lambda}{k^{+}}\right)\left[-\frac{2}{\epsilon}+\ln \left(\frac{\mathbf{k}^{2}}{\mu^{2}}\right)\right]+\frac{\pi^{2}}{3}+2 \ln ^{2}\left(\frac{\Lambda}{k^{+}}\right)\right)\left|g_{i}^{a}(k)\right\rangle .
\end{aligned}
$$

- Two $\boldsymbol{\rho}$ part. This part can be deduced directly from (3.68):

$$
\begin{aligned}
& \left|\psi_{g \rho \rho}^{6}\right\rangle \\
& =-\int_{\Lambda}^{e^{\delta \curlyvee} \Lambda} d k^{+} \int d^{2} \mathbf{k} d^{2} \mathbf{p} \int_{\frac{\Lambda}{k^{+}}}^{1-\frac{\Lambda}{k^{+}}} d \xi \frac{i g^{3} f^{\mathrm{bad}}\left\{\rho^{b}(-\mathbf{p}), \rho^{a}(-\mathbf{k}+\mathbf{p})\right\}}{32 \pi^{9 / 2} \xi(\mathbf{k}-\mathbf{p})^{2}\left((1-\xi) \mathbf{p}^{2}+\xi(\mathbf{k}-\mathbf{p})^{2}\right) \mathbf{k}^{2} \sqrt{k^{+}}} \\
& \times\left[\left(-2(1-\xi) \mathbf{k} \cdot \mathbf{p}+\frac{2\left(1-\xi^{2}\right)}{\xi} \mathbf{p}^{2}\right) \mathbf{k}^{l}+\left(\mathbf{k}^{2}-\frac{1+\xi}{1-\xi}(\mathbf{k}-\mathbf{p})^{2}+\frac{\xi-2}{\xi} \mathbf{p}^{2}\right) \mathbf{p}^{l}\right]\left|g_{l}^{d}(k)\right\rangle .
\end{aligned}
$$

By adding (G.43) with the result which is obtained after changing $p \rightarrow k-p$ and dividing by 2 , we can write $\left|\psi_{g \rho \rho}^{6}\right\rangle$ equivalently as:

$$
\left|\psi_{g \rho \rho}^{6}\right\rangle=-\int_{\Lambda}^{e^{\delta \curlyvee} \Lambda} d k^{+} \int d^{2} \mathbf{k} d^{2} \mathbf{p} \int_{\frac{\Lambda}{k^{+}}}^{1-\frac{\Lambda}{k^{+}}} d \xi \frac{i g^{3} f^{\mathrm{bad}}\left\{\rho^{b}(-\mathbf{p}), \rho^{a}(-\mathbf{k}+\mathbf{p})\right\}}{64 \pi^{9 / 2} \xi(1-\xi) \mathbf{p}^{2}(\mathbf{k}-\mathbf{p})^{2} \mathbf{k}^{2} \sqrt{k^{+}}}
$$




$$
\times\left[\left(-2(1-\xi) \mathbf{k} \cdot \mathbf{p}+\frac{2\left(1-\xi^{2}\right)}{\xi} \mathbf{p}^{2}\right) \mathbf{k}^{l}+\left(\mathbf{k}^{2}-\frac{1+\xi}{1-\xi}(\mathbf{k}-\mathbf{p})^{2}+\frac{\xi-2}{\xi} \mathbf{p}^{2}\right) \mathbf{p}^{l}\right]\left|g_{l}^{d}(k)\right\rangle .
$$

After integration over $\xi$ with the aid of (C.13) and (C.14), the last result becomes:

$$
\begin{aligned}
\left|\psi_{g \rho \rho}^{6}\right\rangle= & -\int_{\Lambda}^{e^{\delta \curlyvee} \Lambda} d k^{+} \int d^{2} \mathbf{k} d^{2} \mathbf{p} \frac{i g^{3} f^{\mathrm{bad}}\left\{\rho^{b}(-\mathbf{p}), \rho^{a}(-\mathbf{k}+\mathbf{p})\right\}}{32 \pi^{9 / 2} \mathbf{p}^{2}(\mathbf{k}-\mathbf{p})^{2} \mathbf{k}^{2} \sqrt{k^{+}}} \\
& \times\left[\ln \left(\frac{\Lambda}{k^{+}}\right) \mathbf{k} \cdot \mathbf{p} \mathbf{k}^{l}+\left(\frac{k^{+}}{\Lambda}-1-\ln \left(\frac{\Lambda}{k^{+}}\right)\right) \mathbf{p}^{2}\left(\mathbf{k}^{l}-\mathbf{p}^{l}\right)\right. \\
& \left.-\left(\ln \left(\frac{\Lambda}{k^{+}}\right) \mathbf{k}^{2}+\left(\frac{k^{+}}{\Lambda}-1-\ln \left(\frac{\Lambda}{k^{+}}\right)\right)(\mathbf{k}-\mathbf{p})^{2}\right) \mathbf{p}^{l}\right]\left|g_{l}^{d}(k)\right\rangle .
\end{aligned}
$$

Notice that under the change $p \rightarrow k-p$ the second summand inside the rectangled brackets becomes exactly the same as the fourth summand. We write the result which is obtained after this change in (3.71).

\section{G.6 Supplement for computation of $\left|\psi_{g}^{7}\right\rangle$}

$\left|\psi_{g}^{7}\right\rangle$ is defined in (3.72). After inserting the matrix elements, (2.49) and (3.23), we arrive at:

$$
\begin{aligned}
\left|\psi_{g}^{7}\right\rangle= & -\frac{1}{2} \int_{\Lambda}^{e^{\delta \curlyvee} \Lambda} d k^{+} d p^{+} d q^{+} d r^{+} \int d^{2} \mathbf{k} d^{2} \mathbf{p} d^{2} \mathbf{q} d^{2} \mathbf{r} \frac{1}{\left(\frac{\mathbf{q}^{2}}{2 q^{+}}\right)\left(\frac{\mathbf{r}^{2}}{2 r^{+}}+\frac{\mathbf{p}^{2}}{2 p^{+}}\right)\left(\frac{\mathbf{k}^{2}}{2 k^{+}}\right)} \\
& \times\left(\delta^{d c} \delta_{k l} \delta^{(3)}(q-k) \frac{g \mathbf{r}^{j} \rho^{b}(\mathbf{r})}{4 \pi^{3 / 2}\left|r^{+}\right|^{3 / 2}}+\delta^{d b} \delta_{j l} \delta^{(3)}(k-r) \frac{g \mathbf{q}^{k} \rho^{c}(\mathbf{q})}{4 \pi^{3 / 2}\left|q^{+}\right|^{3 / 2}}\right) \\
& \times\left(\delta^{a b} \delta_{i j} \delta^{(3)}(r-p) \frac{g \mathbf{q}^{k} \rho^{c}(-\mathbf{q})}{4 \pi^{3 / 2}\left|q^{+}\right|^{3 / 2}}+\delta^{a c} \delta_{i k} \delta^{(3)}(p-q) \frac{g \mathbf{r}^{j} \rho^{b}(-\mathbf{r})}{4 \pi^{3 / 2}\left|r^{+}\right|^{3 / 2}}\right) \\
& \times\left(\frac{g \rho^{a}(-\mathbf{p}) \mathbf{p}^{i}}{4 \pi^{3 / 2}\left|p^{+}\right|^{3 / 2}}\right)\left|g_{l}^{d}(k)\right\rangle .
\end{aligned}
$$

After simplification of the last expression we arrive at:

$$
\begin{aligned}
\left|\psi_{g}^{7}\right\rangle= & -\int_{\Lambda}^{e^{\delta \curlyvee} \Lambda} d k^{+} d p^{+} \int d^{2} \mathbf{k} d^{2} \mathbf{p} \frac{g^{3} \mathbf{p}^{2} \mathbf{k}^{i} \rho^{b}(\mathbf{p})}{64 \pi^{9 / 2}\left(\frac{\mathbf{k}^{2}}{2 k^{+}}\right)\left(\frac{\mathbf{k}^{2}}{2 k^{+}}+\frac{\mathbf{p}^{2}}{2 p^{+}}\right)\left|p^{+}\right|^{3}\left|k^{+}\right|^{3 / 2}} \\
& \times\left(\frac{\rho^{b}(-\mathbf{p}) \rho^{a}(-\mathbf{k})}{\frac{\mathbf{k}^{2}}{2 k^{+}}}+\frac{\rho^{a}(-\mathbf{k}) \rho^{b}(-\mathbf{p})}{\frac{\mathbf{p}^{2}}{2 p^{+}}}\right)\left|g_{i}^{a}(k)\right\rangle .
\end{aligned}
$$

By using the algebra of $\rho$ operators, as in (2.27), we can write the last expression as:

$$
\begin{aligned}
\left|\psi_{g}^{7}\right\rangle= & -\int_{\Lambda}^{e^{\delta \curlyvee} \Lambda} d k^{+} d p^{+} \int d^{2} \mathbf{k} d^{2} \mathbf{p} \frac{2 g^{3} \mathbf{k}^{i} \rho^{b}(\mathbf{p})}{64 \pi^{9 / 2}\left(\frac{\mathbf{k}^{2}}{2 k^{+}}\right)\left|p^{+}\right|^{2}\left|k^{+}\right|^{3 / 2}} \\
& \times\left(\frac{\rho^{b}(-\mathbf{p}) \rho^{a}(-\mathbf{k})}{\frac{\mathbf{k}^{2}}{2 k^{+}}}+\frac{i f^{a b c} \rho^{c}(-\mathbf{k}-\mathbf{p})}{\frac{\mathbf{k}^{2}}{2 k^{+}}+\frac{\mathbf{p}^{2}}{2 p^{+}}}\right)\left|g_{i}^{a}(k)\right\rangle .
\end{aligned}
$$


which we can also write after changing variables as in (3.34) as:

$$
\begin{aligned}
\left|\psi_{g}^{7}\right\rangle= & -\int_{\Lambda}^{e^{\delta \curlyvee} \Lambda} d k^{+} \int d^{2} \mathbf{k} d^{2} \mathbf{p} \int_{\frac{\Lambda}{k^{+}}}^{\frac{e^{\delta \Upsilon} \Lambda}{k^{+}}} d \xi \frac{g^{3} \mathbf{k}^{i} \rho^{b}(\mathbf{p})}{8 \pi^{9 / 2} \sqrt{k^{+}} \xi \mathbf{k}^{2}} \\
& \times\left(\frac{\rho^{b}(-\mathbf{p}) \rho^{a}(-\mathbf{k})}{\xi \mathbf{k}^{2}}+\frac{i f^{a b c} \rho^{c}(-\mathbf{k}-\mathbf{p})}{\xi \mathbf{k}^{2}+\mathbf{p}^{2}}\right)\left|g_{i}^{a}(k)\right\rangle,
\end{aligned}
$$

We can now isolate the contribution to one $\rho$ via the prescription in (3.27).

- One $\rho$ part. Based on (G.48), we see that the term with one $\rho$ operator reads:

$$
\left|\psi_{g \rho}^{7}\right\rangle=\mu^{2 \epsilon} \int_{\Lambda}^{e^{\delta \curlyvee} \Lambda} d k^{+} \int d^{d} \mathbf{k} d^{d} \mathbf{p} \int_{\frac{\Lambda}{k^{+}}}^{\frac{e^{\delta \Upsilon_{\Lambda}}}{k^{+}}} d \xi \frac{g^{3} N_{c} \rho^{a}(-\mathbf{k}) \mathbf{k}^{i}}{16 \pi^{9 / 2} \sqrt{k^{+}} \xi \mathbf{k}^{2}\left(\xi \mathbf{k}^{2}+\mathbf{p}^{2}\right)}\left|g_{i}^{a}(k)\right\rangle .
$$

Integration over $\mathbf{p}$ by using (C.31) yields:

$$
\begin{aligned}
\left|\psi_{g \rho}^{7}\right\rangle= & \mu^{2 \epsilon} \int_{\Lambda}^{e^{\delta \curlyvee} \Lambda} d k^{+} \int \frac{d^{d} \mathbf{k}}{(2 \pi)^{d}} \int_{\frac{\Lambda}{k^{+}}}^{\frac{e^{\delta \curlyvee}}{k^{+}}} d \xi \\
& \times \frac{g^{3} N_{c} \rho^{a}(-\mathbf{k}) \mathbf{k}^{i}}{\sqrt{\pi k^{+}} \xi \mathbf{k}^{2}} \frac{1}{(4 \pi)^{d / 2}} \Gamma\left(1-\frac{d}{2}\right)\left(\frac{1}{\xi \mathbf{k}^{2}}\right)^{1-\frac{d}{2}}\left|g_{i}^{a}(k)\right\rangle .
\end{aligned}
$$

Expanding with the aid of (C.33) taking the $\epsilon \rightarrow 0$ limit and, the last result becomes:

$$
\left|\psi_{g \rho}^{7}\right\rangle=-\int_{\Lambda}^{e^{\delta \curlyvee} \Lambda} d k^{+} \int d^{2} \mathbf{k} \int_{\frac{\Lambda}{k^{+}}}^{\frac{e^{\delta \curlyvee}}{k^{+}}} d \xi \frac{g^{3} N_{c} \rho^{a}(-\mathbf{k}) \mathbf{k}^{i}}{16 \pi^{7 / 2} \sqrt{k^{+}} \xi \mathbf{k}^{2}}\left[-\frac{2}{\epsilon}+\ln \left(\frac{\xi \mathbf{k}^{2}}{\mu_{M S}^{2}}\right)\right]\left|g_{i}^{a}(k)\right\rangle .
$$

After integration over $\xi \equiv \frac{p^{+}}{k^{+}}$according to (C.9):

$$
\begin{aligned}
\left|\psi_{g \rho}^{7}\right\rangle \equiv & -\int_{\Lambda}^{e^{\delta \curlyvee} \Lambda} d k^{+} \int d^{2} \mathbf{k} \frac{g^{3} N_{c} \rho^{a}(-\mathbf{k}) \mathbf{k}^{i}}{32 \pi^{7 / 2} \sqrt{k^{+}} \mathbf{k}^{2}} \\
& \times\left(2 \delta \mathrm{Y}\left[-\frac{2}{\epsilon}+\ln \left(\frac{\mathbf{k}^{2}}{\mu_{\overline{M S}}^{2}}\right)\right]+\ln ^{2}\left(\frac{\Lambda e^{\delta \curlyvee}}{k^{+}}\right)-\ln ^{2}\left(\frac{\Lambda}{k^{+}}\right)\right)\left|g_{i}^{a}(k)\right\rangle .
\end{aligned}
$$

\section{H Supplement for section 4}

\section{H.1 Supplement for computation of $\Sigma_{q \bar{q}}$}

Starting from equation (4.12), and introducing new variables according to (3.34) and (3.45), we obtain:

$$
\begin{aligned}
& \Sigma_{q \bar{q}}^{\mathrm{NLO}}=\int_{\mathbf{x}, \mathbf{y}, \mathbf{z}, \mathbf{z}^{\prime}} \int_{\Lambda}^{e^{\delta \curlyvee} \Lambda} \frac{d k^{+}}{k^{+}} \int_{0}^{1} d \xi \int d^{2} \mathbf{k} d^{2} \widetilde{\mathbf{p}} d^{2} \mathbf{u} d^{2} \widetilde{\mathbf{v}} \\
& \times \frac{g^{4} N_{f} J_{L}^{a}(\mathbf{x}) \operatorname{Tr}\left[S^{\dagger}(\mathbf{z}) t^{a} S\left(\mathbf{z}^{\prime}\right) t^{b}\right] J_{R}^{b}(\mathbf{y})}{512 \pi^{10}\left(\xi(1-\xi) \mathbf{k}^{2}+\widetilde{\mathbf{p}}^{2}\right)\left(\xi(1-\xi) \mathbf{u}^{2}+\widetilde{\mathbf{v}}^{2}\right)}\left(\frac{(1-2 \xi)^{2} \widetilde{\mathbf{p}}^{i} \widetilde{\mathbf{v}}^{j}+\delta^{i j} \widetilde{\mathbf{p}} \cdot \widetilde{\mathbf{v}}-\widetilde{\mathbf{p}}^{j} \widetilde{\mathbf{v}}^{i}}{\mathbf{k}^{2} \mathbf{u}^{2}} \mathbf{k}^{i} \mathbf{u}^{j}\right. \\
& \left.+2 \xi(1-\xi)(1-2 \xi)\left(\frac{\mathbf{k} \cdot \widetilde{\mathbf{p}}}{\mathbf{k}^{2}}+\frac{\mathbf{u} \cdot \widetilde{\mathbf{v}}}{\mathbf{u}^{2}}\right)+4 \xi^{2}(1-\xi)^{2}\right) e^{-i \widetilde{\mathbf{v}} \cdot Z+i \mathbf{u} \cdot\left(Y^{\prime}-\xi Z\right)+i \widetilde{\mathbf{p}} \cdot Z-i \mathbf{k} \cdot\left(X^{\prime}-\xi Z\right)}
\end{aligned}
$$


We can now perform Fourier transformation by using (C.23) - (C.24). It is possible to simplify the denominators using:

$$
\xi(1-\xi) Z^{2}+\left(X^{\prime}-\xi Z\right)^{2}=(1-\xi)\left(X^{\prime}\right)^{2}+\xi X^{2} .
$$

Then, we arrive at:

$$
\begin{aligned}
& \Sigma_{q \bar{q}}^{\mathrm{NLO}}=-\delta \mathrm{Y} \int_{\mathbf{x}, \mathbf{y}, \mathbf{z}, \mathbf{z}^{\prime}} \int_{0}^{1} d \xi \frac{g^{4} N_{f} J_{L}^{a}(\mathbf{x}) \operatorname{Tr}\left[S^{\dagger}(\mathbf{z}) t^{a} S\left(\mathbf{z}^{\prime}\right) t^{b}\right] J_{R}^{b}(\mathbf{y})}{32 \pi^{6} Z^{4}\left((1-\xi)\left(X^{\prime}\right)^{2}+\xi X^{2}\right)\left((1-\xi)\left(Y^{\prime}\right)^{2}+\xi Y^{2}\right)} \\
& \times\left(4 \xi(1-\xi)\left(X^{\prime} \cdot Z-\xi Z^{2}\right)\left(Y^{\prime} \cdot Z-\xi Z^{2}\right)-\left(X^{\prime}-\xi Z\right) \cdot\left(Y^{\prime}-\xi Z\right) Z^{2}\right. \\
& \left.-2 \xi(1-\xi)(1-2 \xi)\left(X^{\prime} \cdot Z+Y^{\prime} \cdot Z-2 \xi Z^{2}\right) Z^{2}-4 \xi^{2}(1-\xi)^{2} Z^{4}\right) .
\end{aligned}
$$

Rewriting the scalar products:

$$
\begin{aligned}
X^{\prime} \cdot Z & =\frac{1}{2}\left(\left(X^{\prime}\right)^{2}-X^{2}+Z^{2}\right), \quad Y^{\prime} \cdot Z=\frac{1}{2}\left(\left(Y^{\prime}\right)^{2}-Y^{2}+Z^{2}\right), \\
X^{\prime} \cdot Y^{\prime} & =\frac{1}{2}\left(\left(X^{\prime}\right)^{2}+\left(Y^{\prime}\right)^{2}-(X-Y)^{2}\right)
\end{aligned}
$$

we arrive at:

$$
\begin{aligned}
\Sigma_{q \bar{q}}^{\mathrm{NLO}}= & -\delta \mathrm{Y} \int_{\mathbf{x}, \mathbf{y}, \mathbf{z}, \mathbf{z}^{\prime}} \int_{0}^{1} d \xi \frac{g^{4} N_{f} J_{L}^{a}(\mathbf{x}) \operatorname{Tr}\left[S^{\dagger}(\mathbf{z}) t^{a} S\left(\mathbf{z}^{\prime}\right) t^{b}\right] J_{R}^{b}(\mathbf{y})}{64 \pi^{6} Z^{4}\left((1-\xi)\left(X^{\prime}\right)^{2}+\xi X^{2}\right)\left((1-\xi)\left(Y^{\prime}\right)^{2}+\xi Y^{2}\right)} \\
& \times\left(-\left[(1-\xi)\left(X^{\prime}\right)^{2}+\xi X^{2}+(1-\xi)\left(Y^{\prime}\right)^{2}+\xi Y^{2}-(X-Y)^{2}\right] Z^{2}\right. \\
& \left.+2 \xi(1-\xi)\left(\left(X^{\prime}\right)^{2}-X^{2}\right)\left(\left(Y^{\prime}\right)^{2}-Y^{2}\right)\right)
\end{aligned}
$$

It is possible to integrate over $\xi$ by using the integrals (C.2), (C.3), and (C.4), the result is shown in (4.15).

\section{H.2 Supplement for computation of $\Sigma_{\text {JSSJ }}$}

Starting from equation (4.16), integrating over $\xi$, and separating the result according to (4.19), we get:

$$
\begin{aligned}
\Sigma_{\mathrm{JSSJ}}^{\mathrm{NLO}}= & \frac{g^{4}}{32 \pi^{6}} \int_{\mathbf{x}, \mathbf{y}, \mathbf{z}, \mathbf{z}^{\prime}} \int_{\Lambda}^{e^{\delta \curlyvee} \Lambda} \frac{d k^{+}}{k^{+}} f^{a b c} f^{d e f} J_{L}^{a}(\mathbf{x}) S_{A}^{b e}(\mathbf{z}) S_{A}^{c f}\left(\mathbf{z}^{\prime}\right) J_{R}^{d}(\mathbf{y}) \\
& \times\left[-\frac{1}{2 Z^{4}}+\frac{\Delta\left(\mathbf{x}, \mathbf{y}, \mathbf{z}, \mathbf{z}^{\prime}\right)}{\left(X^{\prime}\right)^{2} X^{2}\left(\left(X^{\prime}\right)^{2}-X^{2}\right)^{2}\left(\left(X^{\prime}\right)^{2} Y^{2}-X^{2}\left(Y^{\prime}\right)^{2}\right)} \ln \left(\frac{X^{2}}{\left(X^{\prime}\right)^{2}}\right)\right. \\
& \left.-\frac{\Delta\left(\mathbf{y}, \mathbf{x}, \mathbf{z}, \mathbf{z}^{\prime}\right)}{\left(Y^{\prime}\right)^{2} Y^{2}\left(\left(Y^{\prime}\right)^{2}-Y^{2}\right)^{2}\left(\left(X^{\prime}\right)^{2} Y^{2}-X^{2}\left(Y^{\prime}\right)^{2}\right)} \ln \left(\frac{Y^{2}}{\left(Y^{\prime}\right)^{2}}\right)\right],
\end{aligned}
$$

and

$$
\begin{aligned}
\Sigma_{\mathrm{JSSJ}}^{(\delta \mathrm{Y})^{2}}= & -\frac{g^{4}}{32 \pi^{6}} \int_{\mathbf{x}, \mathbf{y}, \mathbf{z}, \mathbf{z}^{\prime}} \int_{\Lambda}^{e^{\delta \curlyvee} \Lambda} \frac{d k^{+}}{k^{+}} f^{a b c} f^{d e f} J_{L}^{a}(\mathbf{x}) S_{A}^{b e}(\mathbf{z}) S_{A}^{c f}\left(\mathbf{z}^{\prime}\right) J_{R}^{d}(\mathbf{y}) \\
& \times\left(\frac{\operatorname{tr}\left(\Lambda\left(\mathbf{x}, \mathbf{z}, \mathbf{z}^{\prime}\right) \Lambda^{T}\left(\mathbf{y}, \mathbf{z}, \mathbf{z}^{\prime}\right)\right)}{\left(X^{\prime}\right)^{2}\left(Y^{\prime}\right)^{2}}+\frac{\operatorname{tr}\left(\Lambda\left(\mathbf{x}, \mathbf{z}^{\prime}, \mathbf{z}\right) \Lambda^{T}\left(\mathbf{y}, \mathbf{z}^{\prime}, \mathbf{z}\right)\right)}{X^{2} Y^{2}}\right) \ln \left(\frac{\Lambda}{k^{+}}\right) .
\end{aligned}
$$


The following definitions were used:

$$
\begin{aligned}
& \Lambda^{j l}\left(\mathbf{x}, \mathbf{z}, \mathbf{z}^{\prime}\right)=\frac{\left(X^{\prime}\right)^{j} Z^{l}}{Z^{2}}+\frac{X^{l}\left(X^{\prime}\right)^{j}}{2 X^{2}}, \quad \Theta^{j l}\left(\mathbf{x}, \mathbf{z}, \mathbf{z}^{\prime}\right)=\frac{X^{2}-\left(X^{\prime}\right)^{2}}{2 Z^{2}} \delta^{j l}, \\
& \begin{array}{l}
\Delta\left(\mathbf{x}, \mathbf{y}, \mathbf{z}, \mathbf{z}^{\prime}\right) \\
=\left(\left(\Lambda^{j l}\left(\mathbf{y}, \mathbf{z}, \mathbf{z}^{\prime}\right) X^{2}+\Lambda^{l j}\left(\mathbf{y}, \mathbf{z}^{\prime}, \mathbf{z}\right)\left(X^{\prime}\right)^{2}\right)\left(X^{2}-\left(X^{\prime}\right)^{2}\right)-\Theta^{j l}\left(\mathbf{y}, \mathbf{z}, \mathbf{z}^{\prime}\right) X^{2}\left(X^{\prime}\right)^{2}\right) \\
\quad \times\left(\left(\Lambda^{j l}\left(\mathbf{x}, \mathbf{z}, \mathbf{z}^{\prime}\right) X^{2}+\Lambda^{l j}\left(\mathbf{x}, \mathbf{z}^{\prime}, \mathbf{z}\right)\left(X^{\prime}\right)^{2}\right)\left(X^{2}-\left(X^{\prime}\right)^{2}\right)-\Theta^{j l}\left(\mathbf{x}, \mathbf{z}, \mathbf{z}^{\prime}\right) X^{2}\left(X^{\prime}\right)^{2}\right) .
\end{array}
\end{aligned}
$$

from which we deduce:

$$
\begin{aligned}
& \frac{\Delta\left(\mathbf{x}, \mathbf{y}, \mathbf{z}, \mathbf{z}^{\prime}\right)}{X^{2}\left(X^{\prime}\right)^{2}\left(X^{2}-\left(X^{\prime}\right)^{2}\right)^{2}}=-\frac{Y^{2}-\left(Y^{\prime}\right)^{2}}{2 Z^{2}\left(X^{2}-\left(X^{\prime}\right)^{2}\right)}\left(\frac{X^{2} X^{\prime} \cdot Z-\left(X^{\prime}\right)^{2} X \cdot Z}{Z^{2}}+X \cdot X^{\prime}\right. \\
& \left.-\frac{X^{2}\left(X^{\prime}\right)^{2}}{Z^{2}}\right)+\frac{1}{\left(X^{\prime}\right)^{2} Z^{2}}\left(\frac{X^{2} Z^{2} X^{\prime} \cdot Y^{\prime}-\left(X^{\prime}\right)^{2} Y^{\prime} \cdot Z X \cdot Z}{Z^{2}}+X^{\prime} \cdot Y^{\prime} X \cdot Z\right. \\
& \left.-Y^{\prime} \cdot Z \frac{X^{2}\left(X^{\prime}\right)^{2}}{2 Z^{2}}\right)-\frac{1}{X^{2} Z^{2}}\left(\frac{X^{2}\left(X^{\prime}\right) \cdot Z Y \cdot Z-\left(X^{\prime}\right)^{2} Z^{2} X \cdot Y}{Z^{2}}+X \cdot Y X^{\prime} \cdot Z\right) \\
& \left.-Y \cdot Z \frac{X^{2}\left(X^{\prime}\right)^{2}}{2 Z^{2}}\right)+\frac{1}{2}\left(\frac{1}{\left(X^{\prime}\right)^{2} Y^{2}}+\frac{1}{X^{2}\left(Y^{\prime}\right)^{2}}\right)\left(\frac{X^{2} X^{\prime} \cdot Y^{\prime} Y \cdot Z-\left(X^{\prime}\right)^{2} X \cdot Y Y^{\prime} \cdot Z}{Z^{2}}\right. \\
& \left.+X \cdot Y X^{\prime} \cdot Y^{\prime}-\frac{X^{2}\left(X^{\prime}\right)^{2}}{2 Z^{2}} Y \cdot Y^{\prime}\right) .
\end{aligned}
$$

We open the brackets and replace the scalar products according to (H.4) and

$$
\begin{aligned}
X \cdot Z & =\frac{1}{2}\left(\left(X^{\prime}\right)^{2}-X^{2}-Z^{2}\right) ; \quad Y \cdot Z=\frac{1}{2}\left(\left(Y^{\prime}\right)^{2}-Y^{2}-Z^{2}\right) ; \\
X \cdot X^{\prime} & =\frac{1}{2}\left(X^{2}+\left(X^{\prime}\right)^{2}-Z^{2}\right) .
\end{aligned}
$$

After these simplifications, the expression (H.10) can be brought to the form:

$$
\begin{aligned}
\frac{\Delta\left(\mathbf{x}, \mathbf{y}, \mathbf{z}, \mathbf{z}^{\prime}\right)}{X^{2}\left(X^{\prime}\right)^{2}\left(X^{2}-\left(X^{\prime}\right)^{2}\right)^{2}} \\
=\frac{1}{8}\left[( X ^ { 2 } ( Y ^ { \prime } ) ^ { 2 } - ( X ^ { \prime } ) ^ { 2 } Y ^ { 2 } ) \left(2 \frac{X^{2}\left(Y^{\prime}\right)^{2}+\left(X^{\prime}\right)^{2} Y^{2}-4(X-Y)^{2} Z^{2}}{Z^{4}\left(X^{2}\left(Y^{\prime}\right)^{2}-\left(X^{\prime}\right)^{2} Y^{2}\right)}\right.\right. \\
\quad+\frac{(X-Y)^{4}}{X^{2}\left(Y^{\prime}\right)^{2}-\left(X^{\prime}\right)^{2} Y^{2}}\left(\frac{1}{X^{2}\left(Y^{\prime}\right)^{2}}+\frac{1}{Y^{2}\left(X^{\prime}\right)^{2}}\right)+\frac{(X-Y)^{2}}{Z^{2}}\left(\frac{1}{X^{2}\left(Y^{\prime}\right)^{2}}-\frac{1}{Y^{2}\left(X^{\prime}\right)^{2}}\right) \\
\left.\quad-\frac{2}{\left(X^{2}-\left(X^{\prime}\right)^{2}\right) Z^{2}}\left(\frac{X^{2}+\left(X^{\prime}\right)^{2}}{Z^{2}}+\frac{Z^{2}-X^{2}}{2\left(X^{\prime}\right)^{2}}+\frac{Z^{2}-\left(X^{\prime}\right)^{2}}{2 X^{2}}-3\right)\right)-\frac{\left(Y^{\prime}\right)^{2}}{Z^{2}} \\
+\frac{\left(Y^{\prime}\right)^{4} X^{2}}{\left(X^{\prime}\right)^{2} Z^{2} Y^{2}}+\frac{Y^{4}\left(X^{\prime}\right)^{2}}{Z^{2} X^{2}\left(Y^{\prime}\right)^{2}}-\frac{Y^{2}}{Z^{2}}-\frac{\left(X^{\prime}\right)^{2} Y^{2}}{Z^{2}\left(Y^{\prime}\right)^{2}}+\frac{X^{2}}{Z^{2}}+\frac{\left(X^{\prime}\right)^{2}}{Z^{2}}-\frac{X^{2}\left(Y^{\prime}\right)^{2}}{Z^{2} Y^{2}} \\
-\frac{\left(X^{\prime}\right)^{2}(X-Y)^{2}}{X^{2} Z^{2}}+\frac{\left(Y^{\prime}\right)^{2}(X-Y)^{2}}{Z^{2} Y^{2}}+\frac{Y^{2}(X-Y)^{2}}{Z^{2}\left(Y^{\prime}\right)^{2}}-\frac{X^{2}(X-Y)^{2}}{\left(X^{\prime} Z^{2}\right.}-\frac{Y^{2}(X-Y)^{2}}{X^{2}\left(Y^{\prime}\right)^{2}} \\
\left.+\frac{(X-Y)^{2}}{\left(X^{\prime}\right)^{2}}+\frac{(X-Y)^{2}}{X^{2}}-\frac{\left(Y^{\prime}\right)^{2}(X-Y)^{2}}{\left(X^{\prime}\right)^{2} Y^{2}}\right] .
\end{aligned}
$$


Comparing (H.12) with (2.59) and noticing that $\widetilde{K}\left(\mathbf{x}, \mathbf{y}, \mathbf{z}, \mathbf{z}^{\prime}\right)$ can be expressed in the following way:

$$
\begin{aligned}
& \widetilde{K}\left(\mathbf{x}, \mathbf{y}, \mathbf{z}, \mathbf{z}^{\prime}\right)=\frac{\alpha_{s}^{2}}{16 \pi^{4}\left(\left(X^{\prime}\right)^{2} Y^{2}-X^{2}\left(Y^{\prime}\right)^{2}\right)}\left(\frac{\left(Y^{\prime}\right)^{2}}{Z^{2}}-\frac{\left(Y^{\prime}\right)^{4} X^{2}}{\left(X^{\prime}\right)^{2} Z^{2} Y^{2}}-\frac{Y^{4}\left(X^{\prime}\right)^{2}}{Z^{2} X^{2}\left(Y^{\prime}\right)^{2}}\right. \\
& +\frac{Y^{2}}{Z^{2}}+\frac{\left(X^{\prime}\right)^{2} Y^{2}}{Z^{2}\left(Y^{\prime}\right)^{2}}-\frac{X^{2}}{Z^{2}}-\frac{\left(X^{\prime}\right)^{2}}{Z^{2}}+\frac{X^{2}\left(Y^{\prime}\right)^{2}}{Z^{2} Y^{2}}+\frac{\left(X^{\prime}\right)^{2}(X-Y)^{2}}{X^{2} Z^{2}}-\frac{\left(Y^{\prime}\right)^{2}(X-Y)^{2}}{Z^{2} Y^{2}} \\
& -\frac{Y^{2}(X-Y)^{2}}{Z^{2}\left(Y^{\prime}\right)^{2}}+\frac{X^{2}(X-Y)^{2}}{\left(X^{\prime}\right)^{2} Z^{2}}+\frac{Y^{2}(X-Y)^{2}}{X^{2}\left(Y^{\prime}\right)^{2}}-\frac{(X-Y)^{2}}{\left(X^{\prime}\right)^{2}}-\frac{(X-Y)^{2}}{X^{2}} \\
& \left.\quad+\frac{\left(Y^{\prime}\right)^{2}(X-Y)^{2}}{\left(X^{\prime}\right)^{2} Y^{2}}\right) \ln \left(\frac{X^{2}}{\left(X^{\prime}\right)^{2}}\right)+(\mathbf{x} \leftrightarrow \mathbf{y}),
\end{aligned}
$$

we arrive at the conclusion that:

$$
\begin{aligned}
& \frac{g^{4}}{32 \pi^{6}}\left[-\frac{1}{2 Z^{4}}+\frac{\Delta\left(\mathbf{x}, \mathbf{y}, \mathbf{z}, \mathbf{z}^{\prime}\right)}{\left(X^{\prime}\right)^{2} X^{2}\left(\left(X^{\prime}\right)^{2}-X^{2}\right)^{2}\left(\left(X^{\prime}\right)^{2} Y^{2}-X^{2}\left(Y^{\prime}\right)^{2}\right)} \ln \left(\frac{X^{2}}{\left(X^{\prime}\right)^{2}}\right)\right. \\
& \left.-\frac{\Delta\left(\mathbf{y}, \mathbf{x}, \mathbf{z}, \mathbf{z}^{\prime}\right)}{\left(Y^{\prime}\right)^{2} Y^{2}\left(\left(Y^{\prime}\right)^{2}-Y^{2}\right)^{2}\left(\left(X^{\prime}\right)^{2} Y^{2}-X^{2}\left(Y^{\prime}\right)^{2}\right)} \ln \left(\frac{Y^{2}}{\left(Y^{\prime}\right)^{2}}\right)\right]=-K_{\text {JSSJ }}\left(\mathbf{x}, \mathbf{y}, \mathbf{z}, \mathbf{z}^{\prime}\right) .
\end{aligned}
$$

By inserting (H.14) to (H.6), it is possible to write the result as in (4.19).

\section{H.3 Supplement for computation of $\Sigma_{\text {JJSJ }}$}

$\Sigma_{\text {JJSJ appears in }}(4.27)$. Here we will present the explict form for $\Sigma_{\text {JJSJ }}^{\text {NLO }}$ and $\left.\Sigma_{\text {JJSJ }}^{(\delta Y}\right)^{2}$ as introduced in (4.28). Let us start with $\Sigma_{\text {JJSJ }}^{\text {NLO }}$

$$
\begin{aligned}
\Sigma_{\mathrm{JJSJ}}^{\mathrm{NLO}} \equiv & -\frac{g^{4} f^{b d e}}{128 \pi^{7}} \delta \mathrm{Y} \int_{\mathbf{w}, \mathbf{x}, \mathbf{y}, \mathbf{z}} \frac{W^{i}}{W^{2}}\left[J_{L}^{d}(\mathbf{x}) J_{L}^{e}(\mathbf{y}) S_{A}^{b a}(\mathbf{z}) J_{R}^{a}(\mathbf{w})\right. \\
& \left.-J_{L}^{a}(\mathbf{w}) S_{A}^{a b}(\mathbf{z}) J_{R}^{d}(\mathbf{x}) J_{R}^{e}(\mathbf{y})\right]\left[\int d^{2} \mathbf{k} d^{2} \mathbf{p} e^{-i \mathbf{k} \cdot X+i \mathbf{p} \cdot(X-Y)}\right. \\
& \left.\times\left(\frac{\mathbf{p}^{i}}{\mathbf{p}^{2}(\mathbf{k}-\mathbf{p})^{2}}-\frac{\mathbf{k}^{i}}{\mathbf{k}^{2}(\mathbf{k}-\mathbf{p})^{2}}+\frac{\mathbf{k}^{i}}{\mathbf{k}^{2} \mathbf{p}^{2}}\right) \ln \left(\frac{(\mathbf{k}-\mathbf{p})^{2}}{\mathbf{k}^{2}}\right)-(X \leftrightarrow Y)\right] .
\end{aligned}
$$

After the change $p \rightarrow k-p$, we find:

$$
\begin{aligned}
\Sigma_{\mathrm{JJSJ}}^{\mathrm{NLO}}= & -\frac{g^{4} f^{b d e}}{128 \pi^{7}} \delta \mathrm{Y} \int_{\mathbf{w}, \mathbf{x}, \mathbf{y}, \mathbf{z}} \frac{W^{i}}{W^{2}}\left[J_{L}^{d}(\mathbf{x}) J_{L}^{e}(\mathbf{y}) S_{A}^{b a}(\mathbf{z}) J_{R}^{a}(\mathbf{w})\right. \\
& \left.-J_{L}^{a}(\mathbf{w}) S_{A}^{a b}(\mathbf{z}) J_{R}^{d}(\mathbf{x}) J_{R}^{e}(\mathbf{y})\right]\left[\int d^{2} \mathbf{k} d^{2} \mathbf{p} e^{-i \mathbf{k} \cdot Y-i \mathbf{p} \cdot(X-Y)}\right. \\
& \left.\left(\frac{\mathbf{k}^{i}-\mathbf{p}^{i}}{\mathbf{p}^{2}(\mathbf{k}-\mathbf{p})^{2}}-\frac{\mathbf{k}^{i}}{\mathbf{k}^{2} \mathbf{p}^{2}}+\frac{\mathbf{k}^{i}}{\mathbf{k}^{2}(\mathbf{k}-\mathbf{p})^{2}}\right) \ln \left(\frac{\mathbf{p}^{2}}{\mathbf{k}^{2}}\right)-(X \leftrightarrow Y)\right] .
\end{aligned}
$$

We can now use integral (C.27), and write:

$$
\begin{aligned}
\Sigma_{\mathrm{JJS} S}^{\mathrm{NLO}}= & \frac{i g^{4} f^{b d e}}{64 \pi^{5}} \delta \mathrm{Y} \int_{\mathbf{w}, \mathbf{x}, \mathbf{y}, \mathbf{z}} \frac{W^{i}}{W^{2}}\left(\frac{X^{i}}{X^{2}}-\frac{Y^{i}}{Y^{2}}\right) \ln \left(\frac{Y^{2}}{(X-Y)^{2}}\right) \ln \left(\frac{X^{2}}{(X-Y)^{2}}\right) \\
& \times\left[J_{L}^{d}(\mathbf{x}) J_{L}^{e}(\mathbf{y}) S_{A}^{b a}(\mathbf{z}) J_{R}^{a}(\mathbf{w})-J_{L}^{a}(\mathbf{w}) S_{A}^{a b}(\mathbf{z}) J_{R}^{d}(\mathbf{x}) J_{R}^{e}(\mathbf{y})\right] .
\end{aligned}
$$


In addition we have:

$$
\begin{aligned}
& \Sigma_{\text {JJSJ }}^{(\delta \mathbf{Y})^{2}} \\
& =\frac{g^{4} f^{b d e}}{256 \pi^{7}}(\delta \mathbf{Y})^{2} \int_{\mathbf{w}, \mathbf{x}, \mathbf{y}, \mathbf{z}} \frac{W^{i}}{W^{2}}\left[J_{L}^{d}(\mathbf{x}) J_{L}^{e}(\mathbf{y}) S_{A}^{b a}(\mathbf{z}) J_{R}^{a}(\mathbf{w})-J_{L}^{a}(\mathbf{w}) S_{A}^{a b}(\mathbf{z}) J_{R}^{d}(\mathbf{x}) J_{R}^{e}(\mathbf{y})\right] \\
& \quad \times\left[\int d^{2} \mathbf{k} d^{2} \mathbf{p} e^{-i \mathbf{k} \cdot X+i \mathbf{p} \cdot(X-Y)}\left(\frac{2 \mathbf{k}^{i}}{\mathbf{k}^{2} \mathbf{p}^{2}}+\frac{\mathbf{p} \cdot(\mathbf{p}-\mathbf{k}) \mathbf{k}^{i}}{\mathbf{k}^{2} \mathbf{p}^{2}(\mathbf{k}-\mathbf{p})^{2}}+\frac{2 \mathbf{p}^{i}}{\mathbf{p}^{2}(\mathbf{k}-\mathbf{p})^{2}}\right)-(X \leftrightarrow Y)\right] .
\end{aligned}
$$

The Fourier transforms can be found in (C.28), (C.29) and (C.30), then we arrive at:

$$
\begin{aligned}
\Sigma_{\mathrm{JJSJ}}^{(\delta \mathrm{Y})^{2}}= & \frac{i g^{4} f^{b d e}}{128 \pi^{6}}(\delta \mathrm{Y})^{2} \int_{\mathbf{w}, \mathbf{x}, \mathbf{y}, \mathbf{z}, \mathbf{z}^{\prime}}\left[\left(\frac{2 X^{\prime} \cdot Y^{\prime} Y \cdot W}{\left(X^{\prime}\right)^{2}\left(Y^{\prime}\right)^{2} Y^{2} W^{2}}+\frac{X^{\prime} \cdot Y^{\prime} Z \cdot W}{\left(X^{\prime}\right)^{2}\left(Y^{\prime}\right)^{2} Z^{2} W^{2}}\right.\right. \\
& \left.\left.+\frac{2 X^{\prime} \cdot Z Y \cdot W}{\left(X^{\prime}\right)^{2} Z^{2} Y^{2} W^{2}}\right)-(X \leftrightarrow Y)\right]\left[J_{L}^{d}(\mathbf{x}) J_{L}^{e}(\mathbf{y}) S_{A}^{b a}(\mathbf{z}) J_{R}^{a}(\mathbf{w})\right. \\
& \left.-J_{L}^{a}(\mathbf{w}) S_{A}^{a b}(\mathbf{z}) J_{R}^{d}(\mathbf{x}) J_{R}^{e}(\mathbf{y})\right] .
\end{aligned}
$$

\section{H.4 Supplement for computation of $\Sigma_{\text {JJSSJJ }}$}

From (4.39) we deduce that:

$$
\begin{aligned}
\Sigma_{\text {JJSSJJ }}= & \int_{\mathbf{w}, \mathbf{v}, \mathbf{x}, \mathbf{y}, \mathbf{z}, \mathbf{z}^{\prime}} \int_{\Lambda}^{e^{\delta \curlyvee} \Lambda} d k^{+} \int_{\frac{\Lambda}{k^{+}}}^{1-\frac{\Lambda}{k^{+}}} d \xi \frac{g^{4}}{128 \pi^{6} \xi(1-\xi)} \\
& \times\left(\frac{X \cdot Y W^{\prime} \cdot V^{\prime}}{X^{2} Y^{2}\left(W^{\prime}\right)^{2}\left(V^{\prime}\right)^{2}} J_{L}^{a}(\mathbf{w}) J_{L}^{b}(\mathbf{x}) S_{A}^{a d}(\mathbf{z}) S_{A}^{b c}\left(\mathbf{z}^{\prime}\right) J_{R}^{d}(\mathbf{v}) J_{R}^{c}(\mathbf{y})\right. \\
& +\frac{X \cdot Y W^{\prime} \cdot V^{\prime}}{X^{2} Y^{2}\left(W^{\prime}\right)^{2}\left(V^{\prime}\right)^{2}} J_{L}^{b}(\mathbf{x}) J_{L}^{a}(\mathbf{w}) S_{A}^{a d}(\mathbf{z}) S_{A}^{b c}\left(\mathbf{z}^{\prime}\right) J_{R}^{d}(\mathbf{v}) J_{R}^{c}(\mathbf{y}) \\
& +\frac{X \cdot Y W^{\prime} \cdot V^{\prime}}{X^{2} Y^{2}\left(W^{\prime}\right)^{2}\left(V^{\prime}\right)^{2}} J_{L}^{a}(\mathbf{w}) J_{L}^{b}(\mathbf{x}) S_{A}^{a d}(\mathbf{z}) S_{A}^{b c}\left(\mathbf{z}^{\prime}\right) J_{R}^{c}(\mathbf{y}) J_{R}^{d}(\mathbf{v}) \\
& \left.+\frac{X \cdot Y W^{\prime} \cdot V^{\prime}}{X^{2} Y^{2}\left(W^{\prime}\right)^{2}\left(V^{\prime}\right)^{2}} J_{L}^{b}(\mathbf{x}) J_{L}^{a}(\mathbf{w}) S_{A}^{a d}(\mathbf{z}) S_{A}^{b c}\left(\mathbf{z}^{\prime}\right) J_{R}^{c}(\mathbf{y}) J_{R}^{d}(\mathbf{v})\right) .
\end{aligned}
$$

Integrating over $\xi$ and reordering the $J$ operators:

$$
\begin{aligned}
\Sigma_{\text {JJSSJJ }}= & \int_{\Lambda}^{e^{\delta \curlyvee} \Lambda} d k^{+} \frac{g^{4}}{64 \pi^{6}} \ln \left(\frac{k^{+}}{\Lambda}\right) \\
& \times\left(\int_{\mathbf{w}, \mathbf{v}, \mathbf{x}, \mathbf{y}, \mathbf{z}, \mathbf{z}^{\prime}} \frac{4 X \cdot Y W^{\prime} \cdot V^{\prime}}{X^{2} Y^{2}\left(W^{\prime}\right)^{2}\left(V^{\prime}\right)^{2}} J_{L}^{a}(\mathbf{w}) J_{L}^{b}(\mathbf{x}) S_{A}^{a d}(\mathbf{z}) S_{A}^{b c}\left(\mathbf{z}^{\prime}\right) J_{R}^{d}(\mathbf{v}) J_{R}^{c}(\mathbf{y})\right. \\
& \left.-\int_{\mathbf{x}, \mathbf{y}, \mathbf{z}, \mathbf{z}^{\prime}} \frac{X \cdot Y X^{\prime} \cdot Y^{\prime}}{X^{2} Y^{2}\left(X^{\prime}\right)^{2}\left(Y^{\prime}\right)^{2}} f^{b a r} f^{c d e} J_{L}^{r}(\mathbf{x}) S_{A}^{b c}\left(\mathbf{z}^{\prime}\right) S_{A}^{a d}(\mathbf{z}) J_{R}^{e}(\mathbf{y})\right)
\end{aligned}
$$

The result after integration over $k^{+}$is shown in equation (4.40).

Open Access. This article is distributed under the terms of the Creative Commons Attribution License (CC-BY 4.0), which permits any use, distribution and reproduction in any medium, provided the original author(s) and source are credited. 


\section{References}

[1] L.V. Gribov, E.M. Levin and M.G. Ryskin, Semihard Processes in QCD, Phys. Rept. 100 (1983) 1 [INSPIRE].

[2] E.A. Kuraev, L.N. Lipatov and V.S. Fadin, The Pomeranchuk Singularity in Nonabelian Gauge Theories, Sov. Phys. JETP 45 (1977) 199 [InSPIRE].

[3] Ya.Ya. Balitsky and L.N. Lipatov, The Pomeranchuk Singularity In Quantum Chromodynamics, Sov. J. Nucl. Phys. 28 (1978) 22.

[4] Y.V. Kovchegov and E. Levin, Quantum Chromodynamics at High Energy, Cambridge University Press, Cambridge U.K. (2012).

[5] E. Iancu and R. Venugopalan, The Color glass condensate and high-energy scattering in $Q C D$, hep-ph/0303204 [INSPIRE].

[6] A.H. Mueller, Small x Behavior and Parton Saturation: A QCD Model, Nucl. Phys. B 335 (1990) 115 [INSPIRE].

[7] A.H. Mueller, Soft gluons in the infinite momentum wave function and the BFKL Pomeron, Nucl. Phys. B 415 (1994) 373 [inSPIRE].

[8] A.H. Mueller, Unitarity and the BFKL Pomeron, Nucl. Phys. B 437 (1995) 107 [hep-ph/9408245] [INSPIRE].

[9] A.H. Mueller and B. Patel, Single and double BFKL Pomeron exchange and a dipole picture of high-energy hard processes, Nucl. Phys. B 425 (1994) 471 [hep-ph/9403256] [INSPIRE].

[10] L.D. McLerran and R. Venugopalan, Computing quark and gluon distribution functions for very large nuclei, Phys. Rev. D 49 (1994) 2233 [hep-ph/9309289] [InSPIRE].

[11] L.D. McLerran and R. Venugopalan, Computing quark and gluon distribution functions for very large nuclei, Phys. Rev. D 49 (1994) 2233 [hep-ph/9309289] [InSPIRE].

[12] I. Balitsky, Operator expansion for high-energy scattering, Nucl. Phys. B 463 (1996) 99 [hep-ph/9509348] [INSPIRE].

[13] I. Balitsky, Factorization for high-energy scattering, Phys. Rev. Lett. 81 (1998) 2024 [hep-ph/9807434] [INSPIRE].

[14] I. Balitsky, Factorization and high-energy effective action, Phys. Rev. D 60 (1999) 014020 [hep-ph/9812311] [INSPIRE].

[15] J. Jalilian-Marian, A. Kovner, A. Leonidov and H. Weigert, The BFKL equation from the Wilson renormalization group, Nucl. Phys. B 504 (1997) 415 [hep-ph/9701284] [INSPIRE].

[16] J. Jalilian-Marian, A. Kovner and H. Weigert, The Wilson renormalization group for low $x$ physics: Gluon evolution at finite parton density, Phys. Rev. D 59 (1998) 014015 [hep-ph/9709432] [INSPIRE].

[17] A. Kovner and J.G. Milhano, Vector potential versus color charge density in low $x$ evolution, Phys. Rev. D 61 (2000) 014012 [hep-ph/9904420] [InSPIRE].

[18] A. Kovner, J.G. Milhano and H. Weigert, Relating different approaches to nonlinear QCD evolution at finite gluon density, Phys. Rev. D 62 (2000) 114005 [hep-ph/0004014] [INSPIRE]. 
[19] A. Kovner, J.G. Milhano and H. Weigert, Relating different approaches to nonlinear QCD evolution at finite gluon density, Phys. Rev. D 62 (2000) 114005 [hep-ph/0004014] [INSPIRE].

[20] E. Iancu, A. Leonidov and L.D. McLerran, Nonlinear gluon evolution in the color glass condensate. 1., Nucl. Phys. A 692 (2001) 583 [hep-ph/0011241] [InSPIRE].

[21] E. Iancu, A. Leonidov and L.D. McLerran, The Renormalization group equation for the color glass condensate, Phys. Lett. B 510 (2001) 133 [hep-ph/0102009] [INSPIRE].

[22] E. Ferreiro, E. Iancu, A. Leonidov and L. McLerran, Nonlinear gluon evolution in the color glass condensate: II, Nucl. Phys. A 703 (2002) 489 [hep-ph/0109115] [INSPIRE].

[23] J. Bartels, High-Energy Behavior in a non-Abelian Gauge Theory (I): $T_{n \rightarrow m}$ in the Leading ln s Approximation, Nucl. Phys. B 151 (1979) 293 [INSPIRE].

[24] J. Bartels, High-Energy Behavior in a Nonabelian Gauge Theory (II). First Corrections to $T_{n \rightarrow m}$ Beyond the Leading $\ln s$ Approximation, Nucl. Phys. B 175 (1980) 365 [INSPIRE].

[25] J. Kwiecinski and M. Praszalowicz, Three Gluon Integral Equation and Odd c Singlet Regge Singularities in QCD, Phys. Lett. B 94 (1980) 413 [InSPIRE].

[26] A. Kovner, E. Levin and M. Lublinsky, QCD unitarity constraints on Reggeon Field Theory, JHEP 08 (2016) 031 [arXiv: 1605.03251] [INSPIRE].

[27] J. Kuokkanen, K. Rummukainen and H. Weigert, HERA-Data in the Light of Small $x$ Evolution with State of the Art NLO Input, Nucl. Phys. A 875 (2012) 29 [arXiv:1108.1867] [INSPIRE].

[28] Y.V. Kovchegov, J. Kuokkanen, K. Rummukainen and H. Weigert, Subleading-N(c) corrections in non-linear small-x evolution, Nucl. Phys. A 823 (2009) 47 [arXiv:0812.3238] [INSPIRE].

[29] J.-P. Blaizot, E. Iancu and H. Weigert, Nonlinear gluon evolution in path integral form, Nucl. Phys. A 713 (2003) 441 [hep-ph/0206279] [INSPIRE].

[30] K. Rummukainen and H. Weigert, Universal features of JIMWLK and BK evolution at small $x$, Nucl. Phys. A 739 (2004) 183 [hep-ph/0309306] [INSPIRE].

[31] A. Dumitru, J. Jalilian-Marian, T. Lappi, B. Schenke and R. Venugopalan, Renormalization group evolution of multi-gluon correlators in high energy QCD, Phys. Lett. B 706 (2011) 219 [arXiv: 1108.4764] [INSPIRE].

[32] Y.V. Kovchegov, Unitarization of the BFKL Pomeron on a nucleus, Phys. Rev. D 61 (2000) 074018 [hep-ph/9905214] [INSPIRE].

[33] E. Gotsman, E. Levin, M. Lublinsky and U. Maor, Towards a new global QCD analysis: Low x DIS data from nonlinear evolution, Eur. Phys. J. C 27 (2003) 411 [hep-ph/0209074] [INSPIRE].

[34] J.L. Albacete, N. Armesto, J.G. Milhano and C.A. Salgado, Non-linear QCD meets data: A Global analysis of lepton-proton scattering with running coupling BK evolution, Phys. Rev. D 80 (2009) 034031 [arXiv:0902.1112] [INSPIRE].

[35] J.L. Albacete, N. Armesto, J.G. Milhano, P. Quiroga-Arias and C.A. Salgado, AAMQS: A non-linear QCD analysis of new HERA data at small-x including heavy quarks, Eur. Phys. J. C 71 (2011) 1705 [arXiv: 1012.4408] [INSPIRE]. 
[36] J.L. Albacete, N. Armesto, J.G. Milhano, C.A. Salgado and U.A. Wiedemann, Numerical analysis of the Balitsky-Kovchegov equation with running coupling: Dependence of the saturation scale on nuclear size and rapidity, Phys. Rev. D 71 (2005) 014003 [hep-ph/0408216] [INSPIRE].

[37] ZEUS, H1 collaboration, F.D. Aaron et al., Combined Measurement and QCD Analysis of the Inclusive e+- $p$ Scattering Cross Sections at HERA, JHEP 01 (2010) 109 [arXiv: 0911.0884] [INSPIRE].

[38] I. Balitsky and G.A. Chirilli, Photon impact factor in the next-to-leading order, Phys. Rev. D 83 (2011) 031502 [arXiv: 1009.4729] [INSPIRE].

[39] M.G. Kozlov, A.V. Reznichenko and V.S. Fadin, Impact factor for gluon production in multi-Regge kinematics in the next-to-leading order, Phys. Atom. Nucl. 75 (2012) 850 [INSPIRE].

[40] G. Beuf, Dipole factorization for DIS at NLO: Loop correction to the $\gamma_{T, L}^{*} \rightarrow q \bar{q}$ light-front wave functions, Phys. Rev. D 94 (2016) 054016 [arXiv: 1606.00777] [INSPIRE].

[41] G. Beuf, NLO corrections for the dipole factorization of DIS structure functions at low $x$, Phys. Rev. D 85 (2012) 034039 [arXiv:1112.4501] [inSPIRE].

[42] G.A. Chirilli, B.-W. Xiao and F. Yuan, Inclusive Hadron Productions in pA Collisions, Phys. Rev. D 86 (2012) 054005 [arXiv: 1203.6139] [INSPIRE].

[43] G.A. Chirilli, B.-W. Xiao and F. Yuan, One-loop Factorization for Inclusive Hadron Production in pA Collisions in the Saturation Formalism, Phys. Rev. Lett. 108 (2012) 122301 [arXiv: 1112.1061] [INSPIRE].

[44] A. Stasto, B.-W. Xiao and F. Yuan, Back-to-Back Correlations of Di-hadrons in dAu Collisions at RHIC, Phys. Lett. B 716 (2012) 430 [arXiv:1109.1817] [InSPIRE].

[45] T. Altinoluk, N. Armesto, G. Beuf, A. Kovner and M. Lublinsky, Single-inclusive particle production in proton-nucleus collisions at next-to-leading order in the hybrid formalism, Phys. Rev. D 91 (2015) 094016 [arXiv:1411.2869] [InSPIRE].

[46] A.M. Stasto and D. Zaslavsky, Saturation in inclusive production beyond leading logarithm accuracy, Int. J. Mod. Phys. A 31 (2016) 1630039 [arXiv:1608.02285] [INSPIRE].

[47] I. Balitsky and G.A. Chirilli, Next-to-leading order evolution of color dipoles, Phys. Rev. D 77 (2008) 014019 [arXiv:0710.4330] [INSPIRE].

[48] I. Balitsky, Quark contribution to the small-x evolution of color dipole, Phys. Rev. D 75 (2007) 014001 [hep-ph/0609105] [INSPIRE].

[49] I.I. Balitsky and A.V. Belitsky, Nonlinear evolution in high density QCD, Nucl. Phys. B 629 (2002) 290 [hep-ph/0110158] [INSPIRE].

[50] V.S. Fadin and L.N. Lipatov, BFKL Pomeron in the next-to-leading approximation, Phys. Lett. B 429 (1998) 127 [hep-ph/9802290] [INSPIRE].

[51] M. Ciafaloni and G. Camici, Energy scale(s) and next-to-leading BFKL equation, Phys. Lett. B 430 (1998) 349 [hep-ph/9803389] [INSPIRE].

[52] Y.V. Kovchegov and H. Weigert, Triumvirate of Running Couplings in Small-x Evolution, Nucl. Phys. A 784 (2007) 188 [hep-ph/0609090] [INSPIRE].

[53] Y.V. Kovchegov and H. Weigert, Quark loop contribution to BFKL evolution: Running 
coupling and leading-N(f) NLO intercept, Nucl. Phys. A 789 (2007) 260 [hep-ph/0612071] [INSPIRE].

[54] E. Gardi, J. Kuokkanen, K. Rummukainen and H. Weigert, Running coupling and power corrections in nonlinear evolution at the high-energy limit, Nucl. Phys. A 784 (2007) 282 [hep-ph/0609087] [INSPIRE].

[55] G.A. Chirilli and Y.V. Kovchegov, Solution of the NLO BFKL Equation and a Strategy for Solving the All-Order BFKL Equation, JHEP 06 (2013) 055 [arXiv:1305.1924] [INSPIRE].

[56] S. Caron-Huot and M. Herranen, High-energy evolution to three loops, arXiv: 1604.07417 [INSPIRE].

[57] A. Kovner, M. Lublinsky and Y. Mulian, Jalilian-Marian, Iancu, McLerran, Weigert, Leonidov, Kovner evolution at next to leading order, Phys. Rev. D 89 (2014) 061704 [arXiv:1310.0378] [INSPIRE].

[58] A. Kovner, M. Lublinsky and Y. Mulian, Conformal symmetry of JIMWLK Evolution at NLO, JHEP 04 (2014) 030 [arXiv: 1401.0374] [INSPIRE].

[59] A. Kovner, M. Lublinsky and Y. Mulian, NLO JIMWLK evolution unabridged, JHEP 08 (2014) 114 [arXiv: 1405.0418] [INSPIRE].

[60] A.V. Grabovsky, Connected contribution to the kernel of the evolution equation for 3-quark Wilson loop operator, JHEP 09 (2013) 141 [arXiv: 1307.5414] [INSPIRE].

[61] I. Balitsky and G.A. Chirilli, Rapidity evolution of Wilson lines at the next-to-leading order, Phys. Rev. D 88 (2013) 111501 [arXiv:1309.7644] [INSPIRE].

[62] S. Caron-Huot, Resummation of non-global logarithms and the BFKL equation, arXiv: 1501.03754 [INSPIRE].

[63] J. Bartels, V.S. Fadin, L.N. Lipatov and G.P. Vacca, NLO Corrections to the kernel of the BKP-equations, Nucl. Phys. B 867 (2013) 827 [arXiv:1210.0797] [InSPIRE].

[64] I. Balitsky and A.V. Grabovsky, NLO evolution of 3-quark Wilson loop operator, JHEP 01 (2015) 009 [arXiv: 1405.0443] [INSPIRE].

[65] A.M. Stasto, B.-W. Xiao and D. Zaslavsky, Towards the Test of Saturation Physics Beyond Leading Logarithm, Phys. Rev. Lett. 112 (2014) 012302 [arXiv:1307.4057] [InSPIRE].

[66] A.M. Stasto, B.-W. Xiao and D. Zaslavsky, Towards the Test of Saturation Physics Beyond Leading Logarithm, Phys. Rev. Lett. 112 (2014) 012302 [arXiv:1307.4057] [INSPIRE].

[67] A. Dumitru, A. Hayashigaki and J. Jalilian-Marian, The Color glass condensate and hadron production in the forward region, Nucl. Phys. A 765 (2006) 464 [hep-ph/0506308] [INSPIRE].

[68] A. Dumitru, K. Dusling, F. Gelis, J. Jalilian-Marian, T. Lappi and R. Venugopalan, The Ridge in proton-proton collisions at the LHC, Phys. Lett. B 697 (2011) 21 [arXiv: 1009.5295] [INSPIRE].

[69] T. Lappi and H. Mäntysaari, Direct numerical solution of the coordinate space Balitsky-Kovchegov equation at next to leading order, Phys. Rev. D 91 (2015) 074016 [arXiv: 1502.02400] [INSPIRE].

[70] K. Kutak and A.M. Stasto, Unintegrated gluon distribution from modified BK equation, Eur. Phys. J. C 41 (2005) 343 [hep-ph/0408117] [INSPIRE].

[71] L. Motyka and A.M. Stasto, Exact kinematics in the small $x$ evolution of the color dipole and gluon cascade, Phys. Rev. D 79 (2009) 085016 [arXiv:0901.4949] [INSPIRE]. 
[72] G. Beuf, Improving the kinematics for low- $x$ QCD evolution equations in coordinate space, Phys. Rev. D 89 (2014) 074039 [arXiv: 1401.0313] [inSPIRE].

[73] E. Iancu, J.D. Madrigal, A.H. Mueller, G. Soyez and D.N. Triantafyllopoulos, Resumming double logarithms in the QCD evolution of color dipoles, Phys. Lett. B 744 (2015) 293 [arXiv: 1502.05642] [INSPIRE].

[74] E. Iancu, J.D. Madrigal, A.H. Mueller, G. Soyez and D.N. Triantafyllopoulos, Collinearly-improved BK evolution meets the HERA data, Phys. Lett. B 750 (2015) 643 [arXiv: 1507.03651] [INSPIRE].

[75] A. Sabio Vera, An 'All-poles' approximation to collinear resummations in the Regge limit of perturbative QCD, Nucl. Phys. B 722 (2005) 65 [hep-ph/0505128] [INSPIRE].

[76] V.N. Gribov and L.N. Lipatov, Deep inelastic e p scattering in perturbation theory, Sov. J. Nucl. Phys. 15 (1972) 438 [InSPIRE].

[77] L.N. Lipatov, ???, Sov. J. Nucl. Phys. 20 (1975) 95.

[78] G. Altarelli and G. Parisi, Asymptotic Freedom in Parton Language, Nucl. Phys. B 126 (1977) 298 [INSPIRE].

[79] Y.L. Dokshitzer, Calculation of the Structure Functions for Deep Inelastic Scattering and $e^{+} e^{-}$Annihilation by Perturbation Theory in Quantum Chromodynamics., Sov. Phys. JETP 46 (1977) 641 [INSPIRE].

[80] G.A. Chirilli, Y.V. Kovchegov and D.E. Wertepny, Classical Gluon Production Amplitude for Nucleus-Nucleus Collisions: First Saturation Correction in the Projectile, JHEP 03 (2015) 015 [arXiv: 1501.03106] [INSPIRE].

[81] A. Kovner, M. Lublinsky and U. Wiedemann, From bubbles to foam: Dilute to dense evolution of hadronic wave function at high energy, JHEP 06 (2007) 075 [arXiv:0705.1713] [INSPIRE].

[82] T. Altinoluk, A. Kovner, M. Lublinsky and J. Peressutti, QCD Reggeon Field Theory for every day: Pomeron loops included, JHEP 03 (2009) 109 [arXiv: 0901.2559] [INSPIRE].

[83] A. Kovner and M. Lublinsky, Remarks on high energy evolution, JHEP 03 (2005) 001 [hep-ph/0502071] [INSPIRE].

[84] A. Kovner and M. Lublinsky, Odderon and seven Pomerons: QCD Reggeon field theory from JIMWLK evolution, JHEP 02 (2007) 058 [hep-ph/0512316] [INSPIRE].

[85] E. Iancu and D.N. Triantafyllopoulos, JIMWLK evolution in the Gaussian approximation, JHEP 04 (2012) 025 [arXiv: 1112.1104] [INSPIRE].

[86] S.J. Brodsky, H.-C. Pauli and S.S. Pinsky, Quantum chromodynamics and other field theories on the light cone, Phys. Rept. 301 (1998) 299 [hep-ph/9705477] [INSPIRE].

[87] R. Venugopalan, Introduction to light cone field theory and high-energy scattering, nucl-th/9808023 [INSPIRE].

[88] D.J. Pritchard and W.J. Stirling, QCD Calculations in the Light Cone Gauge. 1, Nucl. Phys. B 165 (1980) 237 [INSPIRE].

[89] M. Burkardt, Light front quantization, Adv. Nucl. Phys. 23 (1996) 1 [hep-ph/9505259] [INSPIRE].

[90] A. Bassetto, M. Dalbosco, I. Lazzizzera and R. Soldati, Yang-Mills Theories in the Light Cone Gauge, Phys. Rev. D 31 (1985) 2012 [InSPIRE]. 
[91] A. Kovner, High energy evolution: The Wave function point of view, Acta Phys. Polon. B 36 (2005) 3551 [hep-ph/0508232] [INSPIRE].

[92] I. Balitsky and G.A. Chirilli, NLO evolution of color dipoles in $N=4 S Y M$, Nucl. Phys. B 822 (2009) 45 [arXiv:0903.5326] [INSPIRE].

[93] V.S. Fadin, R. Fiore, A.V. Grabovsky and A. Papa, The Dipole form of the gluon part of the BFKL kernel, Nucl. Phys. B 784 (2007) 49 [arXiv:0705.1885] [INSPIRE]. 